\title{
Pt(II)-Coordinated Tricomponent Supramolecular Assemblies of Tetrapyridyl Porphyrin and Dicarboxylate Ligands: Are They 2D Bow Ties or 3D Prisms?
}

\author{
Paola A. Benavides, ${ }^{\dagger}$ Monica A. Gordillo, ${ }^{\dagger}$ Ashok Yadav, ${ }^{\dagger}$ M. Andrey Joaqui-Joaqui, ${ }^{\dagger}$ and Sourav Saha*, ${ }^{\dagger}$ \\ ${ }^{\dagger}$ Department of Chemistry, Clemson University, Clemson, South Carolina 29634, United States \\ †Department of Chemistry, University of Minnesota, Minneapolis, MN 55455, United States \\ *Email: souravs@clemson.edu
}

\begin{abstract}
Thermodynamically favored heteroleptic coordination of one aza- and another oxo-coordinating ligand with Pt(II) ions yield tricomponent supramolecular coordination complexes (SCCs) that have much greater structural complexity and functional diversity than the traditional bicomponent SCCs containing only one of the ligands. Herein, we demonstrate that heteroleptic coordination of tetrapyridyl porphyrins (M'TPP, $\mathrm{M}^{\prime}=\mathrm{Zn}$ or $\mathrm{H}_{2}$ ) and various dicarboxylate ligands (XDC) having different lengths and rigidity with cis$\left(\mathrm{Et}_{3} \mathrm{P}\right)_{2} \mathrm{Pt}^{\mathrm{II}}$ corners actually yields bow tie $(\bowtie)$-shaped tricomponent $\left[\left\{\text { cis- }\left(\mathrm{Et}_{3} \mathrm{P}\right)_{2} \mathrm{Pt}\right\}_{4}\left(\mathrm{M}^{\prime} \mathrm{TPP}\right)(\mathrm{XDC})_{2}\right]^{4+}$ complexes featuring a M'TPP core and two parallel XDC linkers held together by four heteroligated $\mathrm{Pt}^{\mathrm{II}}(\mathrm{N}, \mathrm{O})$ corners. Although previous reports have claimed that the self-assembly of these three components produced tetragonal prisms having two cofacial M'TPP planes connected by four XDC linkers via eight $\mathrm{Pt}^{\mathrm{II}}(\mathrm{N}, \mathrm{O})$ corners, our extensive ${ }^{1} \mathrm{H},{ }^{31} \mathrm{P}$, and $2 \mathrm{D}$ NMR, ESI-MS, X-ray crystallographic, and computational studies unequivocally demonstrated that in reality, no such prism was formed because instead of connecting two cofacial M'TPP ligands, the XDC linkers actually bridged two adjacent pyridyl termini of an M'TPP ligand via shared $\mathrm{Pt}^{\mathrm{II}}(\mathrm{N}, \mathrm{O})$ corners, forming bow tie complexes. In addition to direct crystallographic evidence, the NMR spectra of these complexes revealed that the M'TPP ligands contained two distinct pyrrole protons (4 each) - those located inside the triangles were shielded by and coupled to adjacent XDC linkers, whereas the exposed ones were not - an unmistakable sign of their bow tie structures. Thus, this work not only unveiled novel bow tie-shaped coordination complexes, but also accurately defined the actual structures and compositions of M'TPP-based tricomponent SCCs.
\end{abstract}

\section{INTRODUCTION}

Owing to the dynamic, directional, and self-selecting/rectifying nature of metal-ligand coordination bonds, metal-driven self-assembly processes have emerged as one of the most attractive and versatile tools of supramolecular chemistry, yielding myriads of supramolecular coordination complexes (SCCs) ranging 
from discrete metallacycles ${ }^{1-10}$ and cages ${ }^{11-23}$ to infinite coordination polymers and metal-organic frameworks ${ }^{24-26}$ over the past several decades. To obtain the desired SCCs and to avoid statistical mixtures of different possibilities, typically only one rigid organic ligand is combined with a metal ion at appropriate stoichiometry to obtain bicomponent coordination complexes. Although such two-component selfassembly protocols usually afford the desired SCCs exclusively, they also restrict the structural and functional diversity of the resulting complexes that feature only one organic ligand. Expanding the scope of coordination-driven self-assembly strategies, researchers have recently discovered ${ }^{26-56}$ that cis-capped $^{2}$ $\mathrm{Pt}(\mathrm{II})$ and $\mathrm{Pd}(\mathrm{II})$ corners can simultaneously bind a carboxylate and a pyridyl ligands, preferentially yielding thermodynamically favored heteroleptic $\mathrm{Pt}(\mathrm{N}, \mathrm{O})$ complexes instead of two different homoleptic complexes. ${ }^{29-34,47-53}$ Furthermore, when two different homoleptic $\mathrm{Pt}^{\mathrm{II}}\left(\mathrm{COO}^{-}\right)_{2}$ and $\mathrm{Pt}^{\mathrm{II}}(\text { pyridyl })_{2}$ complexes were mixed together at an appropriate stoichiometry, they spontaneously reorganized into thermodynamically more stable heteroleptic $\mathrm{Pt}(\mathrm{N}, \mathrm{O})$ complexes. ${ }^{30,32,33}$ These revelations paved the door for metal-driven self-assembly of tricomponent metallacycles and cages containing two complementary ligands that further diversified their structures, properties, and functions.

While it is fairly straightforward to assemble 2:2:4 tricomponent rectangles ${ }^{30,32,41}$ containing two parallel dicarboxylate and two parallel dipyridyl arms held together by four heteroligated $\mathrm{Pt}^{\mathrm{II}}(\mathrm{N}, \mathrm{O})$ corners and 2:3:6 trigonal prisms ${ }^{30,32}$ featuring two cofacial trigonal ligands and three ditopic linkers connected by six heteroligated $\mathrm{Pt}^{\mathrm{II}}(\mathrm{N}, \mathrm{O})$ corners, to promote the formation of 2:4:8 tricomponent tetragonal prisms featuring two cofacial tetratopic (tetrapyridyl or tetracarboxylate) ligands and four ditopic (dicarboxylate or dipyridyl) linkers, one must judiciously choose these ligands to ensure that the ditopic linkers can only connect the tips of two cofacial tetratopic ligands via eight heteroleptic Pt(II) corners. ${ }^{30,32,35,36}$ This is extremely important because if a ditopic linker is able to bridge two adjacent binding sites of the tetratopic linker via shared $\mathrm{Pt}(\mathrm{N}, \mathrm{O})$ corners, then they are likely to form 1:2:4 bow tie $(\bowtie)$ complexes instead of 2:4:8 tetragonal prisms via thermodynamically favored heteroleptic coordination since the former would be entropically more favored over the latter.

Nevertheless, Stang and coworkers have recently claimed ${ }^{30,33,34}$ that $\mathrm{Pt}(\mathrm{II})$-driven tricomponent self-assembly of tetrapyridyl porphyrin $\left(\mathrm{M}^{\prime} \mathrm{TPP}, \mathrm{M}^{\prime}=\mathrm{Zn}\right.$ - or $\left.\mathrm{H}_{2}\right)$ and various aromatic and aliphatic dicarboxylate (XDC) linkers with varied length and rigidity yielded 'tetragonal prisms' [\{cis$\left.\left.\left(\mathrm{Et}_{3} \mathrm{P}\right)_{2} \mathrm{Pt}\right\}_{8}\left(\mathrm{M}^{\prime} \mathrm{TPP}\right)_{2}(\mathrm{XDC})_{4}\right]^{8+}$ featuring two parallel $\mathrm{M}^{\prime}$ TTP faces and four XDC pillars held together by eight heteroligated $\mathrm{Pt}^{\mathrm{II}}(\mathrm{N}, \mathrm{O})$ corners. These alleged prisms preserved the photophysical properties of $\mathrm{M}^{\prime}$ TPP chromophores ${ }^{33}$ and displayed promising applications in cancer photodynamic therapy ${ }^{39}$ and guest encapsulation..$^{55}$ Encouraged by these promising literature reports, ${ }^{30,33,34,39,55}$ we attempted to construct bi- 
chromophoric tetragonal prisms composed of two M'TPP faces and four aromatic dicarboxylate linkers having complementary redox- and optically active cores, such as naphthalenediimide and perylene diimide. Surprisingly, none of our attempts to make M'TPP-based bi-chromophoric prisms was successful despite the fact that the XDC linkers were much longer than the distances between the two adjacent pyridyl-N atoms of free M'TPP ligands $\left(d_{\mathrm{N}-\mathrm{N} / \text { free }}=10.9 \AA\right)$, which should have led to the prism formation by eliminating the possibility of intramolecular bridging of two adjacent pyridyl groups via heteroligated $\mathrm{Pt}^{\mathrm{II}}(\mathrm{N}, \mathrm{O})$ corners. Prompted by these unexpected outcomes, we took a closer look at the ${ }^{1} \mathrm{H}$ NMR spectra of M'TPP-based purported tetragonal prisms that were previously reported in the literature. ${ }^{30,33,34,55}$ Interestingly, all these so-called prisms actually displayed ${ }^{30,33,34,55}$ two distinct singlets (1:1 ratio) for the pyrrole protons - one set of four pyrrole protons were significantly more shielded than the other four pyrrole protons (in contrast, all eight pyrrole protons of free M'TPP ligands are chemically equivalent and show one singlet) - indicating that in tricomponent SCCs, the pyrrole rings of M'TPP were no longer chemically equivalent, i.e., they were located in two different environments. These observed ${ }^{1} \mathrm{H}$ NMR signals appeared to be at odds with the proposed tetragonal prism formation because all four pyrrole rings (8Hs) of M'TPP in symmetrical tetragonal prisms should have been located in the same chemical environment and therefore should have displayed the same NMR chemical shift. ${ }^{47-49,53,54}$ On the other hand, the observed NMR signals of two distinct pyrrole protons would actually make sense if two adjacent pyridyl groups of a M'TPP ligand were intramolecularly bridged by two parallel XDC linkers via two shared Pt(II) corners forming 2D bow tie $(\bowtie)$ complexes instead of the proposed 3D prisms. In this scenario, which was previously overlooked, the two opposite pyrrole rings of M'TPP core would be located inside two isosceles triangles formed by two parallel XDC linkers and therefore shielded proportionately by their electron cloud, while the other two opposite pyrrole rings would remain exposed and not shielded directly by the XDC linkers. These inconsistencies prompted us to carefully examine whether or not the $\mathrm{Pt}(\mathrm{II})$-driven self-assembly processes of M'TPP and XDC ligands do actually produce tricomponent prisms or yield an entirely different supramolecular architecture, such as tricomponent bow ties having the same ratio (4:1:2) of the three components.

Herein, we report the self-assembly and in-depth characterization of eight novel bow tie complexes $\left[\left\{\text { cis- }\left(\mathrm{Et}_{3} \mathrm{P}\right)_{2} \mathrm{Pt}\right\}_{4}\left(\mathrm{M}^{\prime} \mathrm{TPP}\right)(\mathrm{XDC})_{2}\right] \cdot 4(\mathrm{TfO})$ composed of $\mathrm{M}^{\prime} \mathrm{TPP}$ ligands $\left(\mathrm{M}^{\prime}=\mathrm{Zn}\right.$ and $\left.\mathrm{H}_{2}\right)$ and four different XDC linkers (Scheme 1). The ${ }^{1} \mathrm{H},{ }^{31} \mathrm{P}$, and 2D (COSY and ROESY) NMR spectra and ESI-MS data of the M'TPP-based tricomponent SCCs presented the telltale signs of 2D bow tie complexes and ruled out prism formation. These compelling, albeit indirect, results suggesting the formation of bow tie complexes were further corroborated by their single-crystal structures, which revealed that each bow tie complex was composed of an M'TPP core and two parallel XDC linkers, which were connected by four heteroligated 
$\left(\mathrm{Et}_{3} \mathrm{P}\right)_{2} \mathrm{Pt}^{\mathrm{II}}(\mathrm{N}, \mathrm{O})$ corners. Notably, this was the first time, single crystal structures of such M'TPP-based tricomponent SCCs could be determined. In addition, the energy-minimized structures of these bow tie complexes were in good agreement with the experimental results, which collectively shined light on why the M'TPP ligands actually formed bow tie complexes instead of tetragonal prisms.

Scheme 1. Pt(II)-driven self-assembly of $M^{\prime}$ TPP ligands $\left(M^{\prime}=\mathrm{Zn}\right.$ or $\left.\mathbf{H}_{2}\right)$ and four different XDC linkers (i.e., 1,6-hexane-, 4,4'-biphenyl-, 1,4-benzene-, and 2,6-naphthalene- dicarboxylates: HDC, BPDC, BDC, and NDC) exclusively yielded novel bow tie-shaped $\left[\left\{c i s-\left(\mathbf{E t}_{3} P\right)_{2} P t\right\}_{4}\left(M^{\prime} T P P\right)(X D C)_{2}\right] \cdot$ 4(TfO) complexes (BT1-BT4 and BT1'-BT4'), each featuring a M'TPP core and two parallel XDC linkers held together by four heteroleptic Pt(II) corners. No tetragonal prism was formed irrespective of the length and rigidity of the XDC linkers.

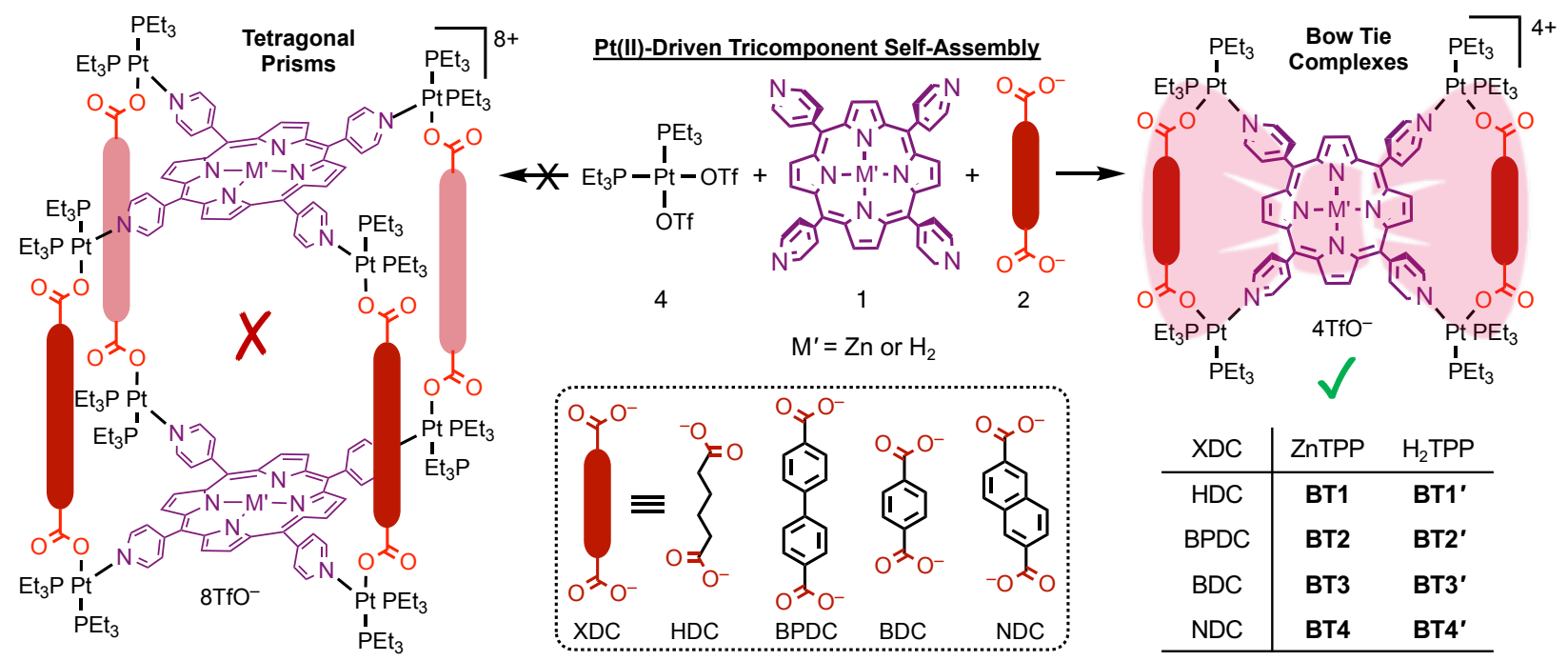

\section{RESULTS AND DISCUSSION}

Heteroleptic Coordination-Driven Self-Assembly of M'TPP-Based Tricomponent Bow Ties ( $\bowtie$ ). Since the heteroleptic coordination of tetratopic M'TPP ligands and ditopic XDC linkers with $c i s-\left(\mathrm{Et}_{3} \mathrm{P}\right)_{2} \mathrm{Pt}^{\mathrm{II}}$ corners could lead to the formation of either (i) tetragonal 3D prisms containing two cofacial M'TPP ligands and four XDC linkers held together by eight Pt(II) corners, ${ }^{30,33,34}$ or (ii) $2 \mathrm{D}$ bow ties containing a M'TPP core and two parallel XDC linkers connected by four shared Pt(II) corners, herein, we decided to determine the actual structures and compositions of the resulting SCCs through extensive multinuclear $\left({ }^{1} \mathrm{H}\right.$ and $\left.{ }^{31} \mathrm{P}\right)$ and 2D (COSY and ROESY) NMR, single-crystal X-ray diffraction, and ESI-MS analyses. To this end, we have employed four different XDC ligands, namely hexanedioate (HDC), 1,4-benzene dicarboxylate (BDC), 2,6-naphthalene dicarboxylate (NDC), and 4,4'-biphenyl dicarboxylate (BPDC) having different 
lengths, rigidity, and $\pi$-electron density in order to determine how they would affect the structures and compositions of the resulting tricomponent SCCs. For consistency, we have adopted the exact same reaction conditions, i.e., a constant 4:1:2 (or 8:2:4) stoichiometry of cis- $\left(\mathrm{Et}_{3} \mathrm{P}\right)_{2} \mathrm{Pt}^{\mathrm{II}}$ corners, $\mathrm{M}^{\prime} \mathrm{TPP}$, and XDC ligands, solvent mixtures $\left(1: 1: 1 \mathrm{CH}_{2} \mathrm{Cl}_{2} / \mathrm{MeCN} / \mathrm{MeNO}_{2}\right.$ or $\left.4: 1 \mathrm{Me}_{2} \mathrm{CO} / \mathrm{H}_{2} \mathrm{O}\right)$ that adequately solubilized all components, temperature $\left(\sim 60^{\circ} \mathrm{C}\right)$, and reaction time $(\sim 18 \mathrm{~h})$ that were also applied by Stang et al..$^{30,33,34}$ in their attempts to assemble $3 \mathrm{D}$ prisms. Although previous reports have claimed that tricomponent selfassembly of HDC and BDC linkers with M'TPP ligand and $\left(\mathrm{Et}_{3} \mathrm{P}\right)_{2} \mathrm{Pt}^{\mathrm{II}}$ corners yielded 3D prisms, ${ }^{30,33,34,39}$ our comprehensive 1D and 2D NMR, ESI-MS, SXRD, and computational studies unequivocally demonstrated that regardless of their length and rigidity, all four XDC linkers employed here, including HDC and BDC, exclusively afforded bow tie architectures, namely ZnTPP-based BT1-BT4 and $\mathrm{H}_{2}$ TPPbased BT1'-BT4' (Scheme 1, Figure 1) instead of any 3D prisms.
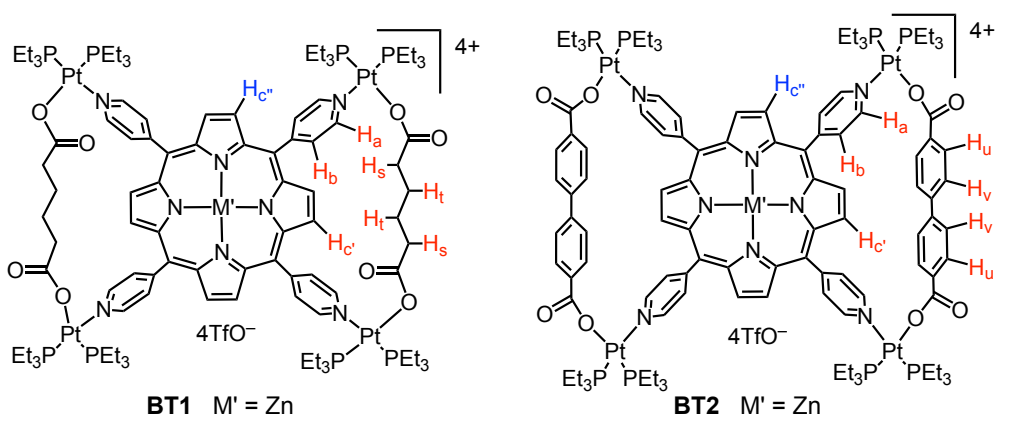

BT1' $\mathrm{M}^{\prime}=\mathrm{H}_{2}$
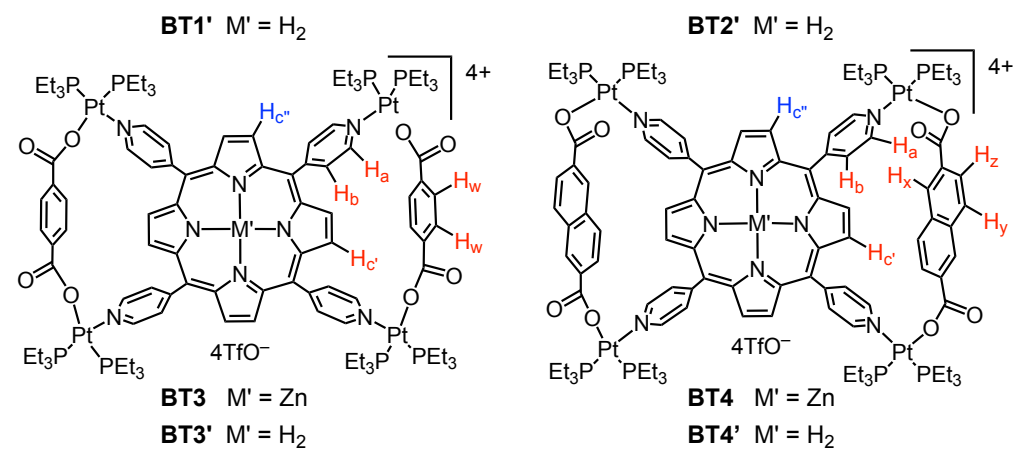

Figure 1. The chemical structures of bow tie complexes.

${ }^{31}$ P NMR Spectroscopy. Simultaneous heteroleptic coordination of a pyridyl group of M'TPP ligand and a carboxylate group of XDC with two cis-Et ${ }_{3} \mathrm{P}$-capped $\mathrm{Pt}^{\mathrm{II}}$ corner was evident from the ${ }^{31} \mathrm{P}$ NMR spectra of all ensuing SCCs (Figures 2 and $\mathrm{S} 1)$. While the cis-( $\left.\mathrm{Et}_{3} \mathrm{P}\right)_{2} \mathrm{Pt}(\mathrm{TfO})_{2}$ precursor displayed a characteristic singlet at $11.14 \mathrm{ppm}$ with two $\mathrm{Pt}(\mathrm{II})$ satellite peaks showing that two $\mathrm{Pt}(\mathrm{II})$-coordinated $\mathrm{P}$ atoms were chemically equivalent, the resulting M'TPP-based SCCs (BT1-BT4 and BT1'-BT4') displayed two distinct doublets, one at ca. $0-2 \mathrm{ppm}$ and another at 6-7 ppm $\left({ }^{2} J_{\mathrm{P}-\mathrm{P}} \approx 20-21 \mathrm{~Hz}\right)$ indicating that the P-atoms 
of two ancillary $\mathrm{Et}_{3} \mathrm{P}$ ligands attached to the square-planar $\mathrm{Pt}(\mathrm{II})$ corners were no longer chemically equivalent. ${ }^{30,33,34}$ The up-field doublets corresponded to the P-nucleus trans- to the pyridyl-N atom of M'TPP, whereas the downfield doublets were attributed to the P-nucleus trans- to the carboxylate O-atom of XDC, which indicated the formation of heteroleptic $c i s-\left(\mathrm{Et}_{3} \mathrm{P}\right)_{2} \mathrm{Pt}^{\mathrm{II}}(\mathrm{N}, \mathrm{O})$ corners. ${ }^{30,33,34}$ The fact that no other ${ }^{31} \mathrm{P}$ NMR signal was observed in any of these cases further confirmed that the tricomponent SCCs were formed exclusively via thermodynamically favored heteroleptic coordination of two different ligands with the Pt(II) corners and no bicomponent complex was formed via homoleptic coordination of two same ligands. Although the ${ }^{31} \mathrm{P}$ NMR data clearly demonstrated simultaneous coordination of carboxylate and pyridyl groups with the $\mathrm{Pt}(\mathrm{II})$ corners, these spectra shed little light on the actual structures and compositions of the resulting tricomponent SCCs, i.e., whether they were actually 3D prisms or 2D bow ties, as both structures would technically feature the same heteroleptic $c i s-\left(\mathrm{Et}_{3} \mathrm{P}\right)_{2} \mathrm{Pt}^{\mathrm{II}}(\mathrm{N}, \mathrm{O})$ corners.

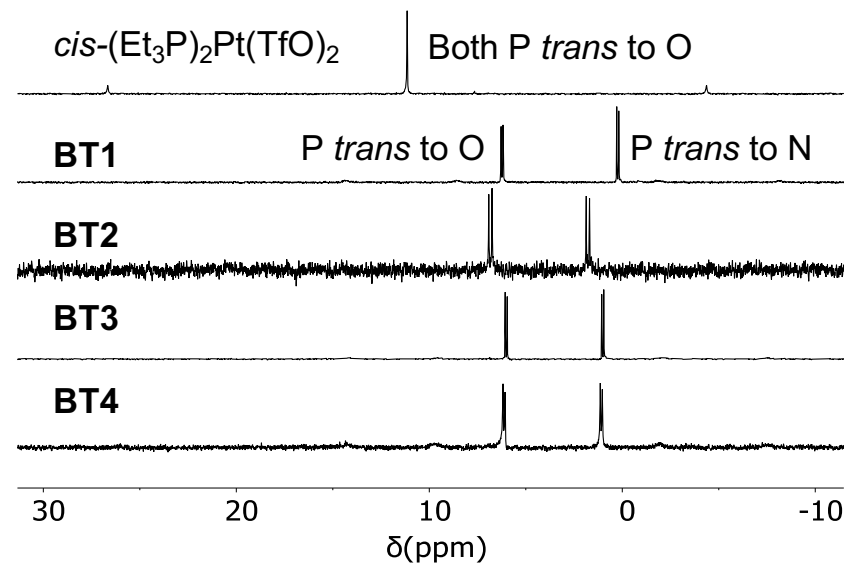

Figure 2. Partial ${ }^{31} \mathrm{P}$ NMR spectra (122 MHz, acetone- $\left.d_{6}\right)$ of $c i s-\left(\mathrm{Et}_{3} \mathrm{P}\right)_{2} \mathrm{Pt}(\mathrm{TfO})$, BT1, BT2, BT3, and BT4 complexes reveal simultaneous coordination of carboxylate and pyridyl groups with heteroligated $\mathrm{Pt}(\mathrm{N}, \mathrm{O})$ corners in the bow tie complexes.

${ }^{1}$ H NMR Spectroscopy. The first telltale signs of the formation of 2D bow tie structures instead of 3D tetragonal prisms came from the ${ }^{1} \mathrm{H}$ NMR spectra of the resulting SCCs (Figures 3, S2, and S3). Not only did all M'TPP-based tricomponent SCCs, i.e., the ZnTPP-based BT1-BT4 and $\mathrm{H}_{2}$ TPP-based BT1'-BT4', display downfield shift of the $\alpha$ - and $\beta$-pyridyl protons $\left(\mathrm{H}_{\mathrm{a}}\right.$ and $\left.\mathrm{H}_{\mathrm{b}}\right)$ of $\mathrm{M}^{\prime} \mathrm{TPP}$ ligand due to $\mathrm{Pt}(\mathrm{II})$ coordination, but most tellingly, they also displayed two distinct singlets (1:1 intensity) representing two different pyrrole protons, i.e., $\mathrm{H}_{\mathrm{c}^{\prime}}$ and $\mathrm{H}_{\mathrm{c}^{\prime \prime}}$ that were no longer chemically equivalent. The splitting and different NMR chemical shifts of two distinct pyrrole protons of M'TPP ligand in the resulting SCCs were the unmistakable signs of bow tie structures, not of tetragonal prisms, as explained below. 


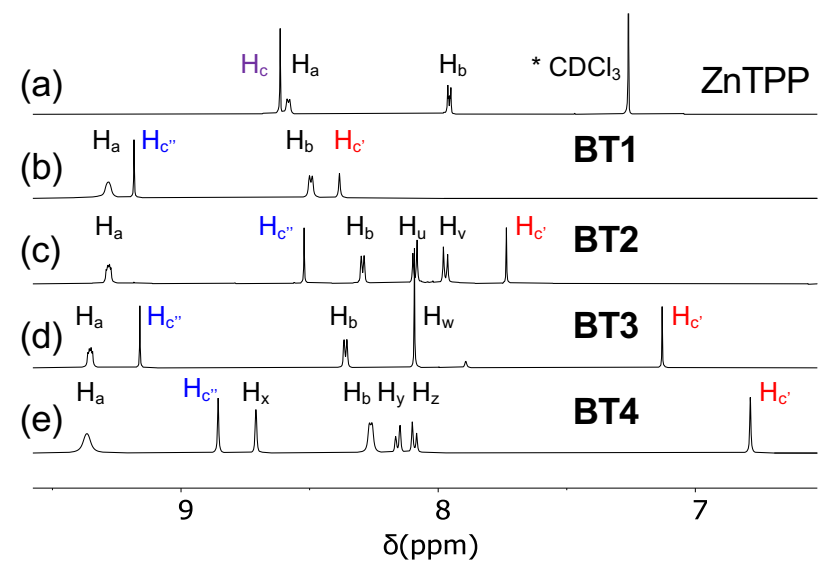

Figure 3. Partial ${ }^{1} \mathrm{H}$ NMR spectra (500 MHz) of (a) free $\mathrm{ZnTPP}$ ligand in $\mathrm{CDCl}_{3}$, (b) BT1, (c) BT2, (d) BT3, and (e) BT4 complexes (in acetone- $d_{6}$ ) show that the enclosed $\mathrm{H}_{\mathrm{c}^{\prime}}$ pyrrole protons (highlighted in red) of bow tie complexes are shifted up-field commensurately with the shielding effect of the adjacent XDC linkers, whereas the chemical shifts of exposed $\mathrm{H}_{\mathrm{c}^{\prime \prime}}$ pyrrole protons (highlighted in blue) vary depending on the length of XDC linkers that dictate their distances from the adjacent pyridyl rings.

The $D_{4 \mathrm{~h}}$-symmetric free ZnTPP ligand displayed two doublets at 8.58 and $7.95 \mathrm{ppm}$ corresponding to its $\mathrm{H}_{\mathrm{a}}$ and $\mathrm{H}_{\mathrm{b}}$ pyridyl protons ( 8 each) and a sharp singlet at $8.61 \mathrm{ppm}$ for all eight chemically equivalent $\mathrm{H}_{\mathrm{c}}$ pyrrole protons (Figure 3). In the resulting tricomponent SCCs, the $\mathrm{H}_{\mathrm{a}}$ and $\mathrm{H}_{\mathrm{b}}$ peaks shifted noticeably downfield to ca. 9.3 and $8.4 \mathrm{ppm}$, respectively, due to Pt(II)-coordination. More importantly, while all eight $\mathrm{H}_{\mathrm{c}}$ pyrrole protons of free ZnTPP were chemically equivalent (s, $\left.8.61 \mathrm{ppm}\right)$, BT1-BT4 complexes displayed two distinct singlets (1:1 intensity) for $\mathrm{H}_{\mathrm{c}^{\prime}}$ and $\mathrm{H}_{\mathrm{c}^{\prime \prime}}$ pyrrole protons that were no longer chemically equivalent. The chemical shifts of $\mathrm{H}_{\mathrm{c}^{\prime}}$ and $\mathrm{H}_{\mathrm{c}^{\prime \prime}}$ pyrrole protons varied depending on the length and $\pi$-electron density, or lack thereof, of the XDC linkers, confirming that they were located in completely different chemical environments that were controlled by the XDC ligands. The singlet peaks corresponding to four $\mathrm{H}_{\mathrm{c}^{\prime}}$ pyrrole protons of BT1-BT4 complexes appeared at 8.38, 7.73, 7.13, and 6.79 ppm, respectively, which were significantly up-field shifted from the $\mathrm{H}_{\mathrm{c}}$ signal of free ZnTPP, while the singlets corresponding to the other four $\mathrm{H}_{\mathrm{c}^{\prime \prime}}$ pyrrole protons appeared at 9.18, 8.52, 9.16, and $8.86 \mathrm{ppm}$, respectively. The up-field shifts of $\mathrm{H}_{\mathrm{c}^{\prime}}$ signals of BT1-BT4 was directly correlated to the increasing $\pi$-electron cloud and shielding effect of XDC linkers (HDC $<$ BPDC $<$ BDC $<$ NDC), suggesting that these pyrrole protons were located inside the triangles of the bow tie structures $(\bowtie)$ formed by two parallel XDC linkers while the less shielded and down-field $\mathrm{H}_{\mathrm{c}^{\prime \prime}}$ pyrrole protons were not. Naturally, the aliphatic HDC linkers in BT1 exerted the least shielding effect on the enclosed $\mathrm{H}_{\mathrm{c}^{\prime}}$ pyrrole protons located across the bay, causing the smallest up-field shift, followed by the BPDC linkers of BT2 having two twisted phenyl rings that were not properly aligned 
with the enclosed $\mathrm{H}_{\mathrm{c}^{\prime}}$ pyrrole protons according to its single-crystal structure (vide infra). On the other hand, the enclosed $\mathrm{H}_{\mathrm{c}^{\prime}}$ pyrrole protons of BT3 and BT4 experienced much greater up-field NMR chemical shifts because they were projected toward the aromatic cores of BDC and NDC linkers, as seen from their crystal structures (vide infra), which exerted much greater shielding effects. Thus, having the largest $\pi$-cloud and maximum shielding effect among all four XDC linkers employed here, the NDC linkers caused the largest up-field shift of the $\mathrm{H}_{\mathrm{c}^{\prime}}$ signal in BT4, followed by the BDC linkers in BT3.

With respect to the enclosed $\mathrm{H}_{\mathrm{c}^{\prime}}$ pyrrole protons located inside the triangles of bow tie complexes, which displayed up-field shift commensurate with the shielding effect of XDC linkers, the four exposed $\mathrm{H}_{\mathrm{c}^{\prime \prime}}$ pyrrole protons of BT1-BT4 complexes appeared at downfield positions $(9.18,8.52,9.16$, and 8.86 ppm, respectively), indicating that they were not shielded by XDC linkers (Figure 3). However, the chemical shifts of exposed $\mathrm{H}_{\mathrm{c}^{\prime \prime}}$ pyrrole protons were still affected by the length of the XDC linkers, which controlled their distances from the adjacent pyridyl rings of ZnTPP and thus their variable shielding effects. This was further evident from their crystal structures presented below and certain structural parameters summarized in Table 1. For instance, in order to form the triangles of bow tie structures $(\bowtie)$, the shorter HDC and BDC linkers $\left(l_{\mathrm{BDC}}=6.9 \AA\right)$ of BT1 and BT3 complexes, respectively, pulled the two XDCbridged Pt(II) corners closer to each other (Table 1), which in turn, pulled the pyridyl rings of ZnTPP ligand away from the exposed $\mathrm{H}_{\mathrm{c}^{\prime \prime}}$ pyrrole protons. This reduced the shielding effect of pyridyl rings on $\mathrm{H}_{\mathrm{c}^{\prime \prime}}$ pyrrole protons in BT1 and BT3, causing them to appear at more downfield positions (ca. $9.2 \mathrm{ppm}$ ) than the $\mathrm{H}_{\mathrm{c}}$ protons of free ZnTPP ligand. Having a medium length $\left(l_{\mathrm{NDC}}=9.2 \AA\right)$, the NDC linkers of BT4 also pulled the pyridyl rings slightly away from the exposed $\mathrm{H}_{\mathrm{c}^{\prime \prime}}$ pyrrole protons, causing a slight downfield shift from the $\mathrm{H}_{\mathrm{c}}$ signal of free ZnTPP. On the other hand, the longest BPDC linkers $\left(l_{\mathrm{BPDC}}=11.2 \AA\right)$ of BT2 pushed the two bridged Pt(II) corners away from each other, which in turn, pushed the pyridyl rings of ZnTPP closer to the exposed $\mathrm{H}_{\mathrm{c}^{\prime \prime}}$ pyrrole protons making them more shielded and up-field shifted than the $\mathrm{H}_{\mathrm{c}}$ protons of free ZnTPP.

Similarly, the $\mathrm{H}_{2}$ TPP-based BT1'-BT4' complexes also displayed ${ }^{1} \mathrm{H}$ NMR spectra (Figure S2) that were fully consistent with bow tie structures, but not with tetragonal prisms. Just as in ZnTPP-based BT1-BT4, in these complexes, the $\mathrm{H}_{\mathrm{a}}$ and $\mathrm{H}_{\mathrm{b}}$ pyridyl protons of $\mathrm{H}_{2}$ TPP ligand shifted downfield due to Pt(II) coordination, whereas chemically non-equivalent $\mathrm{H}_{\mathrm{c}^{\prime}}$ and $\mathrm{H}_{\mathrm{c}^{\prime \prime}}$ pyrrole protons (4 each) displayed two distinct singlets. Again, the up-field chemical shifts of $\mathrm{H}_{\mathrm{c}^{\prime}}$ pyrrole protons $-8.35 \mathrm{ppm}$ in HDC-based BT1', 7.74 ppm BPDC-based BT2', 7.08 ppm in BDC-based BT2', and 6.78 ppm in NDC-based BT4' - were dictated by the shielding effect of the XDC linkers, indicating that these $\mathrm{H}_{2}$ TPP protons were located inside the triangles formed by two parallel XDC linkers. On the other hand, the NMR chemical shifts of $\mathrm{H}_{\mathrm{c}^{\prime \prime}}$ 
pyrrole protons varied based on the length of the XDC linkers (not their $\pi$-clouds), which in turn defined the distance between these protons and the adjacent pyridyl rings and thereby controlled their shielding effect. The short HDC and BDC linkers in BT1' and $\mathbf{B T 3}^{\prime}$ pulled two adjacent pyridyl rings coordinated to the bridged $\mathrm{Pt}(\mathrm{II})$ corners closer to each other and away from the exposed $\mathrm{H}_{\mathrm{c}^{\prime \prime}}$ pyrrole protons, causing downfield shift (9.38 and 9.31 ppm, respectively). On the other hand, the long BPDC linkers in BT2' pushed two adjacent pyridyl rings coordinated to the bridged Pt(II) corners away from each other and closer to the exposed $\mathrm{H}_{\mathrm{c}^{\prime \prime}}$ pyrrole protons, which caused an up-field shift $(8.58 \mathrm{ppm})$. The medium length NDC linkers in BT4' caused the least deviation of the pyridyl rings from their original positions, allowing the exposed $\mathrm{H}_{\mathrm{c}^{\prime \prime}}$ pyrrole protons to appear $(8.85 \mathrm{ppm})$ close to the original position of $\mathrm{H}_{\mathrm{c}}$ protons of free $\mathrm{H}_{2} \mathrm{TPP}$.

Thus, the splitting of pyrrole protons of M'TPP ligands into two sets of chemically non-equivalent $\mathrm{H}_{\mathrm{c}^{\prime}}$ and $\mathrm{H}_{\mathrm{c}^{\prime \prime}}$ protons (4 each) in the tricomponent SCCs and the up-field shift of the $\mathrm{H}_{\mathrm{c}^{\prime}}$ signal depending on the electron cloud and shielding effect of XDC linkers clearly indicated the formation of 2D bow tie structures BT1-BT4 and BT1'-BT4' where two opposite pyrrole rings of M'TPP ligand bearing the $\mathrm{H}_{\mathrm{c}^{\prime}}$ protons were located inside the triangles formed by two parallel XDC linkers while the other two pyrrole rings bearing the $\mathrm{H}_{\mathrm{c}^{\prime \prime}}$ protons remained exposed. It is worth noting that tricomponent SCCs have been assembled previously ${ }^{33}$ under the same conditions using the same cis- $\left(\mathrm{Et}_{3} \mathrm{P}\right)_{2} \mathrm{Pt}^{\mathrm{II}}$ corner, $\mathrm{M}^{\prime} \mathrm{TPP}$, and HDC and BDC ligands, which displayed the same ${ }^{1} \mathrm{H}$ NMR spectra as those displayed by BT1, BT1', BT2, and BT2' featuring two distinct singlets for $\mathrm{H}_{\mathrm{c}^{\prime}}$ and $\mathrm{H}_{\mathrm{c}^{\prime \prime}}$ pyrrole protons along with the doublets for $\mathrm{H}_{\mathrm{a}}$ and $\mathrm{H}_{\mathrm{b}}$ pyridyl protons and the characteristic signals of XDC protons. ${ }^{33}$ However, these telltale signs of 2D bow tie structures were overlooked previously, which led to a mischaracterization of these four 2D bow tie complexes as 3D prisms. If tricomponent self-assembly of $\left(\mathrm{Et}_{3} \mathrm{P}\right)_{2} \mathrm{Pt}^{\mathrm{II}}, \mathrm{M}^{\prime} \mathrm{TPP}$, and $\mathrm{XDC}$ linkers had indeed formed tetragonal prisms having two cofacial M'TPP planes connected by four XDC linkers via heteroleptic $\left(\mathrm{Et}_{3} \mathrm{P}\right)_{2} \mathrm{Pt}^{\mathrm{II}}(\mathrm{N}, \mathrm{O})$ corners, as previously claimed with $\mathrm{HDC}, \mathrm{BDC}$, and few other dicarboxylate linkers, ${ }^{30,33,34,55}$ then all sixteen pyrrole protons of two parallel M'TPP faces should have remained chemically equivalent and displayed one singlet peak ${ }^{47-49,53,54}$ instead of splitting into two chemically nonequivalent $\mathrm{H}_{\mathrm{c}^{\prime}}$ and $\mathrm{H}_{\mathrm{c}^{\prime \prime}}$ protons that showed two distinct singlets. However, that was not observed for any of the Pt(II)/M'TPP/XDC-based tricomponent SCCs presented either here or in the literature, ${ }^{30,33,34,55}$ which ruled out the prism formation.

2D ${ }^{1} \mathbf{H}-{ }^{1} \mathbf{H}$ COSY NMR Spectroscopy. The 2D COSY NMR spectra of these TTP-based tricomponent SCCs (Figure S4) provided further insights into their actual structures by revealing the coupling between the adjacent $\alpha$ - and $\beta$-protons. In addition to showing COSY couplings between $\mathrm{H}_{\mathrm{a}}$ and $\mathrm{H}_{\mathrm{b}}$ pyridyl protons 
of M'TPP ligands, BT1, BT2, and BT4 (and BT1', BT2', and BT4') complexes also displayed couplings between $\mathrm{H}_{\mathrm{s}}$ and $\mathrm{H}_{\mathrm{t}}$ protons of $\mathrm{HDC}, \mathrm{H}_{\mathrm{u}}$ and $\mathrm{H}_{\mathrm{v}}$ protons of BPDC, and $\mathrm{H}_{\mathrm{x}}, \mathrm{H}_{\mathrm{y}}$ and $\mathrm{H}_{\mathrm{z}}$ protons of NDC linkers, respectively. Conspicuously missing from the COSY NMR spectra of all these SCCs were any such $\alpha / \beta$ proton coupling between the $\mathrm{H}_{\mathrm{c}^{\prime}}$ and $\mathrm{H}_{\mathrm{c}^{\prime \prime}}$ pyrrole protons of $\mathrm{M}^{\prime}$ TPP ligand, which further indicated that these two chemically non-equivalent protons did not belong to the same pyrrole ring but to two distinct pyrrole rings located in different environments. This scenario would be possible only in bow tie structures where two opposite pyrrole rings carrying the $\mathrm{H}_{\mathrm{c}^{\prime}}$ protons resided inside the triangles formed by two parallel XDC linkers, whereas the other two pyrrole rings bearing $\mathrm{H}_{\mathrm{c}^{\prime \prime}}$ protons remained exposed, eliminating the possibility of any $\alpha / \beta$-coupling between these two remote protons. If in fact these tricomponent SCCs were 3D prisms containing two cofacial M'TPP panels linked by four XDC linkers, then not only should all the pyrrole protons of M'TPP have remained chemically equivalent instead of splitting into distinct $\mathrm{H}_{\mathrm{c}^{\prime}}$ and $\mathrm{H}_{\mathrm{c}^{\prime \prime}}$ protons, but at the very least, each pyrrole ring would have carried one $\mathrm{H}_{\mathrm{c}^{\prime}}$ and one $\mathrm{H}_{\mathrm{c}^{\prime \prime}}$ protons, which would have shown $\alpha / \beta$-coupling. The absence of such couplings ruled out the prism formation.

2D ROESY NMR Spectroscopy. Further corroborating evidence of the formation of bow tie complexes instead of 3D prisms stemmed from the ROESY NMR spectra of M'TPP-based tricomponent SCCs (Figures 4 and S5), which revealed the through-space long-range ${ }^{1} \mathrm{H}-{ }^{1} \mathrm{H}$ coupling between the enclosed $\mathrm{H}_{\mathrm{c}^{\prime}}$ pyrrole protons of M'TPP located inside the triangles and the XDC protons located across the bay. No such coupling between the exposed $\mathrm{H}_{\mathrm{c}^{\prime \prime}}$ pyrrole protons of $\mathrm{M}^{\prime} \mathrm{TPP}$ and the remote XDC protons were observed (the $\mathrm{H}_{\mathrm{c}^{\prime \prime}}$ protons were only coupled to $\mathrm{H}_{\mathrm{b}}$ protons of the adjacent pyridyl ring in some cases), further verifying that the enclosed $\mathrm{H}_{\mathrm{c}^{\prime}}$ and exposed $\mathrm{H}_{\mathrm{c}^{\prime \prime}}$ pyrrole protons were located in two completely different chemical environments, which would be only possible in bow tie structures, not in prisms.

In BT1 and BT1', the enclosed $\mathrm{H}_{\mathrm{c}^{\prime}}$ pyrrole protons of $\mathrm{M}^{\prime} \mathrm{TPP}$ were $\mathrm{ROE}$-coupled with the $\mathrm{H}_{\mathrm{t}}$ protons of $\mathrm{HDC}$ linker and the $\mathrm{H}_{\mathrm{b}}$ protons of the adjacent pyridyl ring (Figures $4 \mathrm{a}$ and $\mathrm{S} 5 \mathrm{a}$ ), whereas the $\mathrm{H}_{\mathrm{c}^{\prime \prime}}$ protons were coupled to only $\mathrm{H}_{\mathrm{b}}$ protons of the adjacent pyridyl ring, not with any HDC protons. These observations further confirmed that the $\mathrm{H}_{\mathrm{c}^{\prime}}$ protons were located inside the triangles formed by two parallel HDC linkers, which enabled their coupling, while the exposed $\mathrm{H}_{\mathrm{c}^{\prime \prime}}$ protons were located far away from the HDC linkers. These couplings were also fully consistent with a bow tie structure, not with a $3 \mathrm{D}$ prism architecture containing two ZnTPP faces and four HDC linkers, which was proposed previously. ${ }^{33}$ If the purported prism had been really formed, then $\mathrm{H}_{\mathrm{c}^{\prime}}$ and $\mathrm{H}_{\mathrm{c}^{\prime \prime}}$ protons would have remained chemically equivalent, and both should have been coupled to the HDC protons in a similar fashion. The absence of these signs further ruled out the prism formation. 

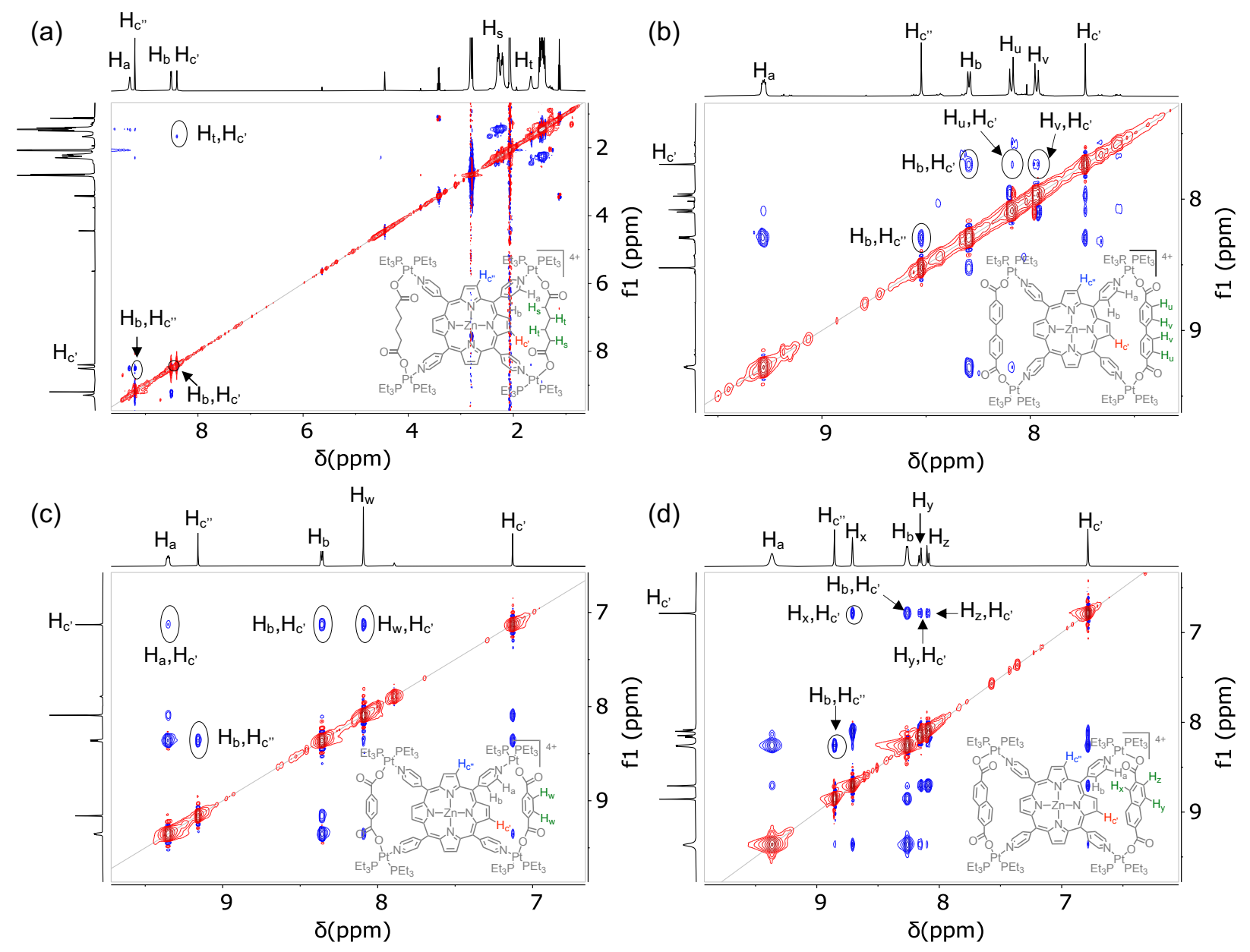

Figure 4. Partial ${ }^{1} \mathrm{H}-{ }^{1} \mathrm{H}$ ROESY NMR spectra (500 MHz, acetone- $d_{6}$ ) of (a) BT1, (b) BT2, (c) BT3, and (d) BT4 show that the enclosed $\mathrm{H}_{\mathrm{c}^{\prime}}$ pyrrole protons of these bow tie complexes located inside the isosceles triangles are through-space NOE-correlated with the protons of adjacent XDC linkers but the exposed $\mathrm{H}_{\mathrm{c}^{\prime \prime}}$ pyrrole protons are not coupled with the distant XDC protons.

Similarly, in BT2 and BT2', the enclosed $\mathrm{H}_{\mathrm{c}^{\prime}}$ pyrrole protons of $\mathrm{M}^{\prime} \mathrm{TPP}$ were coupled with the $\mathrm{H}_{\mathrm{u}}$ and $\mathrm{H}_{\mathrm{v}}$ protons of BPDC linkers located across the bay (Figures $4 \mathrm{~b}$ and $\mathrm{S} 5 \mathrm{~b}$ ) as well as with the $\mathrm{H}_{\mathrm{b}}$ protons of the adjacent pyridyl ring, whereas the exposed $\mathrm{H}_{\mathrm{c}^{\prime \prime}}$ protons were coupled to only adjacent $\mathrm{H}_{\mathrm{b}}$ protons, not with any BPDC protons. These correlations further confirmed that the $\mathrm{H}_{\mathrm{c}^{\prime}}$ pyrrole protons were located inside the triangles formed by two parallel BPDC ligands, while the exposed $\mathrm{H}_{\mathrm{c}^{\prime \prime}}$ protons were located far away from these linkers, a scenario that was possible only in bow tie structures.

In BT3 and BT3', the enclosed $\mathrm{H}_{\mathrm{c}^{\prime}}$ pyrrole protons of $\mathrm{M}^{\prime}$ TPP were coupled with the $\mathrm{H}_{\mathrm{w}}$ protons of BDC linkers (Figures $4 \mathrm{c}$ and S5c), indicating that they were located inside the triangles formed by the BDC 
linkers, and also with the $\mathrm{H}_{\mathrm{b}}$ protons of the adjacent pyridyl rings because the shorter BDC linkers pulled the bridged Pt(II) corners closer to each other and consequently brought the pyridyl rings closer to enclosed $\mathrm{H}_{\mathrm{c}^{\prime}}$ pyrrole protons. In contrast, the exposed $\mathrm{H}_{\mathrm{c}^{\prime \prime}}$ pyrrole protons were not coupled with any BDC protons, which were located far away. These coupling patterns were also consistent with bow tie structures, not with the previously proposed 3D prism architectures containing two M'TPP faces and four BDC linkers held together by eight heteroligated $\mathrm{Pt}(\mathrm{II})$ corners. If the purported prism had been formed, then the $\mathrm{H}_{\mathrm{c}^{\prime}}$ and $\mathrm{H}_{\mathrm{c}^{\prime \prime}}$ protons should have remained chemically equivalent, and both should also have been coupled with the BDC protons $\left(\mathrm{H}_{\mathrm{w}}\right)$ in a similar fashion. In the absence of such signals, the prism formation can be ruled out. Notably, not only did the ${ }^{1} \mathrm{H}$ NMR spectra of previously reported M'TPP-based SCCs containing BDC and HDC linkers not reconcile with the purported prism structures (vide supra), but also no 2D NMR and/or xray crystallographic evidence was presented that could have supported that claim. ${ }^{30,33,34,39,55,56}$

Finally, in BT4 and BT4', the enclosed $\mathrm{H}_{\mathrm{c}^{\prime}}$ pyrrole protons of $\mathrm{M}^{\prime} \mathrm{TPP}$ were coupled with the $\mathrm{H}_{\mathrm{x}}$, $\mathrm{H}_{\mathrm{y}}$, and $\mathrm{H}_{\mathrm{z}}$ protons of NDC linkers (Figures $4 \mathrm{~d}$ and $\mathrm{S} 5 \mathrm{~d}$ ), indicating that they were part of the same triangle. The $\mathrm{H}_{\mathrm{c}^{\prime}}$ pyrrole protons were also coupled with the $\mathrm{H}_{\mathrm{b}}$ protons of the adjacent pyridyl rings, as the NDC linkers pulled the bridged $\mathrm{Pt}(\mathrm{II})$ corners closer to each other and thereby brought the pyridyl rings closer to the $\mathrm{H}_{\mathrm{c}^{\prime}}$ protons. On the other hand, the exposed $\mathrm{H}_{\mathrm{c}^{\prime \prime}}$ pyrrole protons were not coupled with any NDC protons, indicating that they were located far away from each other. In addition, the $\mathrm{H}_{\mathrm{a}}$ protons of $\mathrm{ZnTPP}$ and $\mathrm{H}_{\mathrm{x}}$ and $\mathrm{H}_{\mathrm{y}}$ of NDC, which were part of the same triangles, were also coupled. Once again, these NOEcorrelations were fully consistent with bow tie structures, not with the prisms where all pyrrole protons should have remained chemically equivalent and coupled with the NDC protons in a similar fashion.

ESI-MS Analysis. The ESI-MS analysis revealed the characteristic $m / z$ peaks of dicationic $[\mathrm{M}-2 \mathrm{TfO}]^{2+}$ species of bow tie complexes having a general formula of $\left[\left\{\left(\mathrm{Et}_{3} \mathrm{P}\right)_{2} \mathrm{Pt}\right\}_{4}\left(\mathrm{M}^{\prime} \mathrm{TPP}\right)(\mathrm{XDC})_{2}\right]^{4+} \bullet 4\left(\mathrm{TfO}^{-}\right)$. The ZnTPP-based bow ties BT1, BT2, BT3, and BT4 displayed (Figures 5 and S6) the respective $[\mathrm{M}-2 \mathrm{TfO}]^{2+}$ peaks at $m / z=1497.09,1593.58,1517.01$, and 1567.05, while the $\mathrm{H}_{2}$ TPP-bow ties BT2', BT3', and BT4' displayed (Figure S6) their respective $[\mathrm{M}-2 \mathrm{TfO}]^{2+}$ peaks at $m / z=1561.50,1485.52$, and 1535.60. Although all of these M'TPP-based tricomponent bow tie complexes were assembled under exactly the same conditions as those reported in the literature, none of them displayed any ESI-MS peak that would otherwise indicate the formation of tetragonal prisms. 

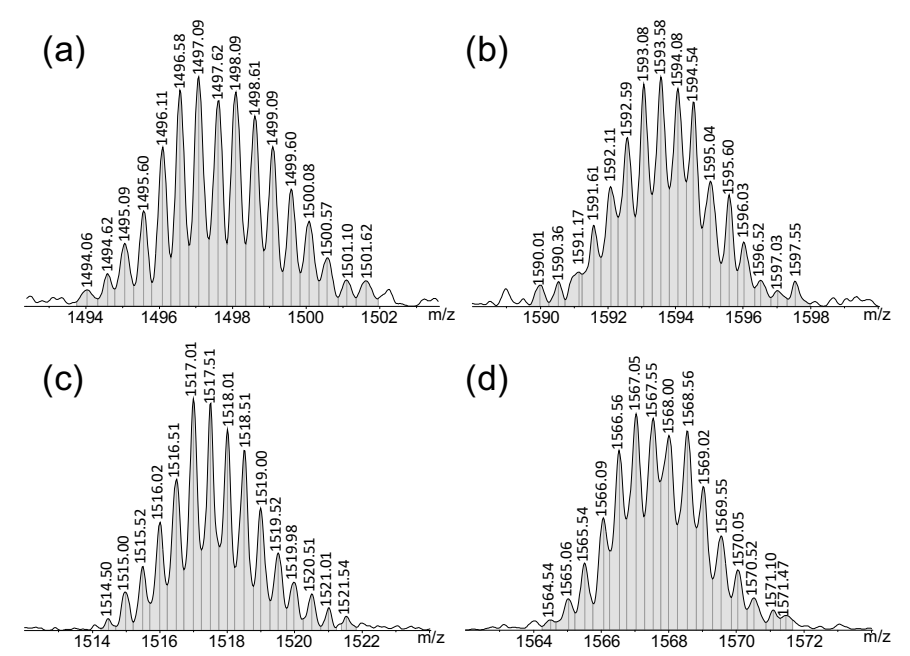

Figure 5. ESI-MS data show the $[\mathrm{M}-2 \mathrm{TfO}]^{2+}$ peaks of (a) BT1, (b) BT2, (c) BT3, and (d) BT4 complexes.

Apparently, the $m / z$ peaks of $\left[\mathrm{M}-\mathrm{nX}^{-}\right]^{\mathrm{n}+}$ species $\left(\mathrm{X}^{-}=\mathrm{TfO}^{-}\right.$or $\mathrm{PF}_{6}^{-}, \mathrm{n}=3$ or 5$)$ of the purported $\left[\left\{\left(\mathrm{Et}_{3} \mathrm{P}\right)_{2} \mathrm{Pt}\right\}_{8}\left(\mathrm{M}^{\prime} \mathrm{TPP}\right)_{2}(\mathrm{XDC})_{4}\right]^{8+} \bullet 8\left(\mathrm{X}^{-}\right)$prisms containing HDC and BDC linkers were observed previously. ${ }^{33}$ However, on a rare occasion when the entire ESI-MS profile of a tricomponent SCC assembled from ( $\left.\mathrm{Et}_{3} \mathrm{P}\right)_{2} \mathrm{Pt}^{\mathrm{II}}, \mathrm{H}_{2} \mathrm{TPP}$, and $\mathrm{BDC}$ was disclosed previously ${ }^{30}$ (not just certain blown-up peaks), it revealed a prominent $\mathrm{m} / \mathrm{z}$ peak at $1481.38(\sim 100 \%$ relative intensity, $4-5$ times higher than any other peaks in the entire panel) that actually represented the $\left[\mathrm{M}-2 \mathrm{PF}_{6}{ }^{-}\right]^{2+}$ species of bow tie complex BT3': $^{\prime}$ $\left[\left\{\left(\mathrm{Et}_{3} \mathrm{P}\right)_{2} \mathrm{Pt}\right\}_{4}\left(\mathrm{H}_{2} \mathrm{TPP}\right)(\mathrm{BDC})_{2}\right]^{4+} \bullet 4\left(\mathrm{PF}_{6}{ }^{-}\right)$. Just like the diagnostic ${ }^{1} \mathrm{H}$ NMR signals of bow tie complexes $\left(\mathrm{H}_{\mathrm{c}^{\prime}}\right.$ and $\mathrm{H}_{\mathrm{c}^{\prime \prime}}$ signals), this characteristic ESI-MS peak of BT3' was also overlooked previously, ${ }^{30}$ which unfortunately led to mischaracterization of M'TPP-based tricomponent 4:1:2 bow tie complexes as 8:2:4 prisms. Since none of the previously reported M'TPP-based tricomponent SCCs (i.e., so-called prisms) displayed the ${ }^{1} \mathrm{H}$ NMR spectra that actually supported the alleged prism formation (vide supra), ${ }^{30,33,34,55,56}$ and our extensive 2D NMR and ESI-MS data discussed above as well as the single-crystal structures presented below unequivocally demonstrated the formation of bow tie complexes instead of any prisms, it is plausible that the $m / z$ peaks that were previously ascribed to $8: 2: 4\left[\left\{\left(\mathrm{Et}_{3} \mathrm{P}\right)_{2} \mathrm{Pt}\right\} 8\left(\mathrm{M}^{\prime} \mathrm{TPP}\right)_{2}(\mathrm{XDC})_{4}\right]^{8+} \bullet 8\left(\mathrm{X}^{-}\right.$ ) prisms, were actually associated with the dimers of 4:1:2 $\left[\left\{\left(\mathrm{Et}_{3} \mathrm{P}\right)_{2} \mathrm{Pt}\right\}_{4}\left(\mathrm{M}^{\prime} \mathrm{TPP}\right)(\mathrm{XDC})_{2}\right]^{4+} \bullet 4\left(\mathrm{X}^{-}\right)$bow tie complexes (after loss of some counterions) instead of the purported prisms. Alternatively, the selfassembled bow tie complexes may have reorganized into prisms under certain electrospray ionization conditions, giving a false impression about the alleged prisms formation that was not otherwise supported by any other experimental data. Therefore, it was risky and misleading to define the structures of M'TPPbased tricomponent SCCs primarily based on the ESI-MS analysis especially when their ${ }^{1} \mathrm{H}$ NMR spectra were not at all consistent with those assignments. 
Single-Crystal X-Ray Structures of Tricomponent Bow Tie ( $\bowtie$ ) Complexes. Finally, single-crystal Xray diffraction analysis presented the most direct evidence of bow tie structures of M'TPP-based tricomponent SCCs. Notably, this is the first time the crystal structures of any M'TPP-based tricomponent SCCs could be determined, which together with the NMR and ESI-MS data unequivocally confirmed their bow tie structures and ruled out any prism formation. Some of these bow tie complexes crystallized directly from the NMR solutions (acetone- $d_{6}$ ), while others formed crystals upon vapor diffusion of $\mathrm{CH}_{2} \mathrm{Cl}_{2}$ or $\mathrm{Et}_{2} \mathrm{O}$ into NMR solutions. Although we were able to obtain the crystals of ZnTPP-based BT2, BT3, and BT4, and $\mathrm{H}_{2}$ TPP-based BT2' and BT3' containing rigid aromatic BPDC, BDC, and NDC linkers, it was not possible to grow crystals of BT1 and BT1' complexes containing flexible aliphatic HDC linkers. The structural features of all bow tie complexes (Table 1) were fully consistent with their respective ${ }^{1} \mathrm{H}$ and ${ }^{31} \mathrm{P}$ NMR spectra, 2D ${ }^{1} \mathrm{H}-{ }^{1} \mathrm{H}$ NMR (COSY and ROESY) correlations, and ESI-MS data, confirming that the same species were present both in solutions and in solid crystals.
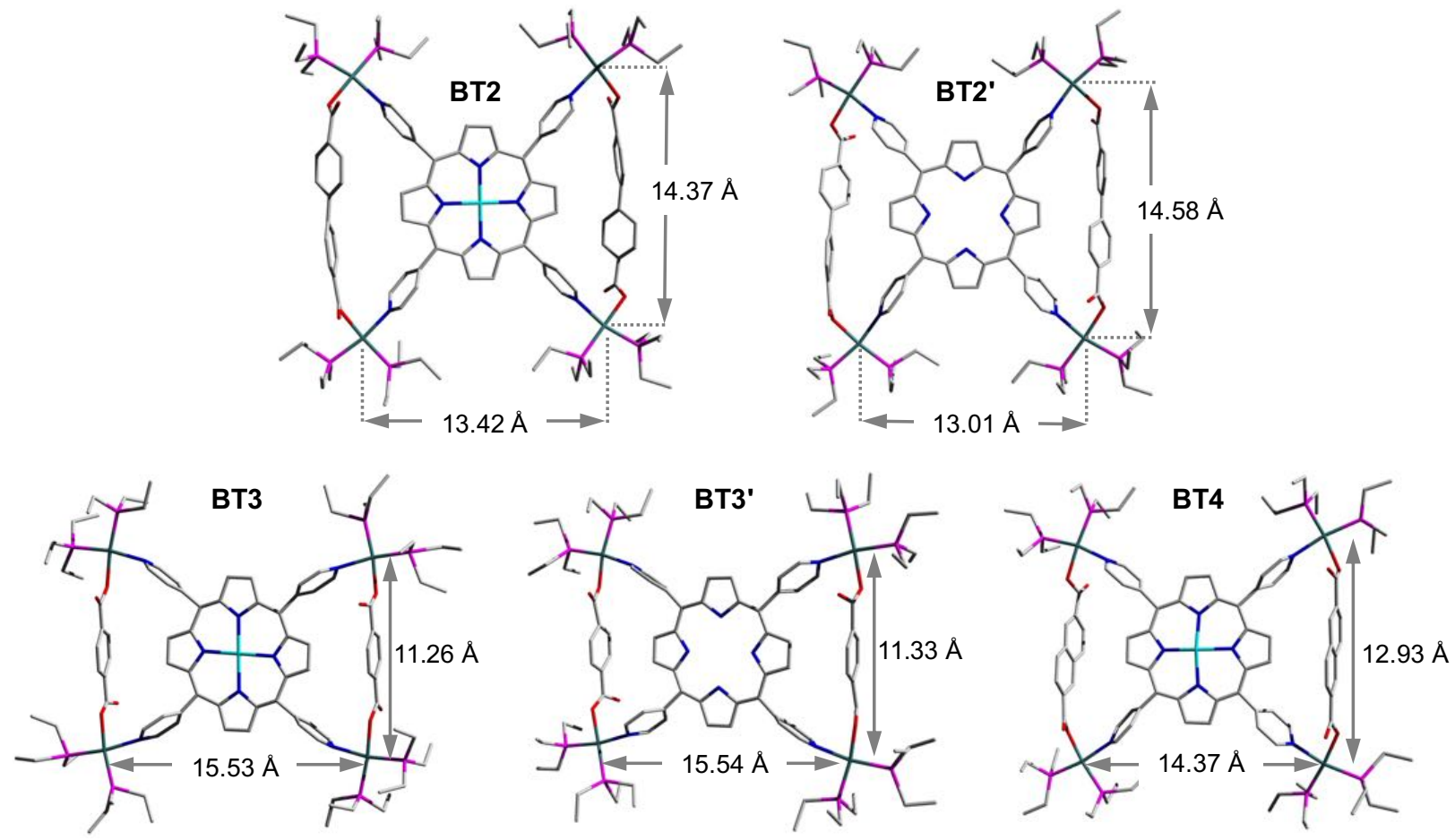

Figure 6. Single-crystal structures of BT2, BT2', BT3, BT3' and BT4 bow tie complexes. The distances between adjacent Pt(II) centers bridged and not bridged by XDC linkers ( $d_{\mathrm{Pt}-\mathrm{Pt} / \text { int }}$ and $\left.d_{\mathrm{Pt} \text {-Pt/ext }}\right)$ are labeled to show the differences and how that affected the shapes of the M'TPP ligands. Atom legend: green: Pt, cyan: Zn, pink: $\mathrm{P}$, red: $\mathrm{O}$, blue: $\mathrm{N}$, grey: $\mathrm{C}$. The $\mathrm{H}$-atoms and $\mathrm{TfO}^{-}$anions were omitted for clarity. 
Table 1. Key structural parameters of bow tie complexes obtained from their single-crystal structures. The parameters obtained from the calculated structures are labeled with asterisks $\left({ }^{*}\right)$.

\begin{tabular}{|c|c|c|c|c|c|c|}
\hline & BT1 calc. ${ }^{*}$ & BT2 & BT2' $^{\prime}$ & BT3 (calc. ${ }^{*}$ ) & BT3' (calc.*) & BT4 \\
\hline Space Group & - & $P \overline{1}$ & $C 2 / \mathrm{c}$ & $C 2 / \mathrm{c}$ & $14_{1} / \mathrm{a}$ & $P 2_{1} / \mathrm{c}$ \\
\hline$d_{\mathrm{Pt}-\mathrm{Pt} \text { int }}(\AA)$ & $11.55^{*}$ & 14.37 & 14.58 & $11.26\left(11.33^{*}\right)$ & $11.33\left(11.33^{*}\right)$ & 12.93 \\
\hline$d_{\mathrm{Pt}-\mathrm{Pt} / \mathrm{ext}}(\AA)$ & $15.41^{*}$ & 13.42 & 13.01 & $15.53\left(15.59^{*}\right)$ & $15.54\left(15.56^{*}\right)$ & 14.37 \\
\hline$d_{N-N / i n t}(\AA)$ & $10.19^{*}$ & 11.38 & 11.52 & $10.09\left(10.12^{*}\right)$ & $10.07\left(10.20^{*}\right)$ & 10.83 \\
\hline$d_{N-N / e x t}(\AA)$ & $11.63^{*}$ & 10.37 & 10.18 & $11.39\left(11.69^{*}\right)$ & $11.61\left(11.68^{*}\right)$ & 10.80 \\
\hline$d_{\mathrm{Hc}^{\prime}-\mathrm{XDC} \text {-center }}(\AA)$ & $2.84^{*}$ & 3.37 & 3.11 & $3.18\left(3.50^{\star}\right)$ & $3.05\left(3.44^{*}\right)$ & 2.95 \\
\hline$d_{\mathrm{Hc}^{\prime}-\text { Pyridine-center }}(\AA)$ & $2.96^{*}$ & 3.41 & 3.51 & $3.02\left(2.98^{*}\right)$ & $2.96\left(3.01^{*}\right)$ & 3.19 \\
\hline$d_{H c^{\prime \prime}-\text { Pyridine-center }}(\AA)$ & $3.37^{*}$ & 3.08 & 3.00 & $3.41\left(3.47^{*}\right)$ & $3.47\left(3.41^{*}\right)$ & 3.26 \\
\hline$\angle \mathrm{N}-\mathrm{Pt}-\mathrm{O}\left({ }^{\circ}\right)$ & $96^{*}, 98^{*}$ & 82,85 & 82,83 & $78,83\left(93^{*}\right)$ & $80,83\left(93^{*}\right)$ & 82,82 \\
\hline$\angle\left(\mathrm{N}_{\mathrm{Py}}-\text { Center- }-\mathrm{N}_{\mathrm{Py}}\right)_{\text {int }}\left({ }^{\circ}\right)$ & $82^{*}$ & 95 & 97 & $83\left(81^{*}\right)$ & $82\left(82^{*}\right)$ & 88 \\
\hline$\angle\left(\mathrm{N}_{\mathrm{Py}}-\text { Center- }-\mathrm{N}_{\mathrm{Py}}\right)_{\mathrm{ext}}\left({ }^{\circ}\right)$ & $98^{*}$ & 85 & 83 & $97\left(99^{*}\right)$ & $98\left(98^{*}\right)$ & 89 \\
\hline$\theta_{\text {Porphyrin/Pyridyl-dh }}\left({ }^{\circ}\right)$ & $82^{*}, 83^{*}$ & 76,77 & 64,73 & $86,88\left(84^{*}\right)$ & $76,80\left(85^{\star}\right)$ & 65,76 \\
\hline$\theta_{\text {Pyrrole/XDC-dh }}\left({ }^{\circ}\right)$ & - & - & - & $80\left(90^{*}\right)$ & $85\left(90^{*}\right)$ & 87 \\
\hline
\end{tabular}

The bow tie $(\bowtie)$-shaped BT2 and BT2' complexes (Figure 6: $\left[\left\{\left(\mathrm{Et}_{3} \mathrm{P}\right)_{2} \mathrm{Pt}\right\}_{4}\left(\mathrm{M}^{\prime} \mathrm{TPP}\right)(\mathrm{BPDC})_{2}\right]^{4+}, \mathrm{M}^{\prime}$ $=\mathrm{Zn}$ or $\mathrm{H}_{2}$ ) crystallized in $P \overline{1}$ and $C 2 / \mathrm{c}$ space groups, respectively. Each complex consisted of a M'TPP core and two parallel BPDC linkers held together by four heteroleptic $\left(\mathrm{Et}_{3} \mathrm{P}\right)_{2} \mathrm{Pt}(\mathrm{N}, \mathrm{O})$ corners. The $\angle \mathrm{N}-\mathrm{Pt}-$ $\mathrm{O}$ angles of slightly distorted square-planar Pt(II) corners ranged between ca. $82-85^{\circ}$ (two diagonally opposite angles were the same), slightly less than an ideal $90^{\circ}$ bond angle. In BT2, the distances between two adjacent Pt(II) corners bridged by a BPDC linker $\left(d_{\mathrm{Pt}-\mathrm{Pt} / \text { int }}\right)$ were $14.4 \AA$ (14.6 $\AA$ in BT2'), whereas the distances between two adjacent Pt(II) corners not bridged by a BPDC linker $\left(d_{\text {Pt-Pt/ext }}\right)$ were $13.4 \AA(13.0 \AA$ in BT2'). This happened because the long BPDC linker $\left(l_{\mathrm{BPDC}}=11.2 \AA\right)^{57}$ placed the two bridged $\operatorname{Pt}(\mathrm{II})$ corners farther away from each other, which in turn shortened the distances between the two adjacent $\operatorname{Pt}($ II) corners that were not bridged by BPDC. Consequently, the distance between the two adjacent pyridyl-N atoms coordinated to two BPDC-bridged Pt(II) corners, i.e., the pyridyl-N atoms that belonged to the same triangle $\left(d_{\mathrm{N}-\mathrm{N} / \mathrm{int}}=11.4 \AA\right.$ in $\mathbf{B T} 2$ and $11.5 \AA$ in $\left.\mathbf{B T 2} 2^{\prime}\right)$, were noticeably longer than the distance between two adjacent pyridyl-N atoms that were not part of the same triangle $\left(d_{\mathrm{N}-\mathrm{N} / \mathrm{ext}}=10.4 \AA\right.$ in BT2 and $10.2 \AA$ in BT2'). As a result, the N-N distances in BT2 and BT2' complexes were different from the uniform distance between two adjacent pyridyl-N atoms of free M'TPP ligands $\left(d_{\mathrm{N}-\mathrm{N} / \text { free }} \approx 10.9 \AA\right) .{ }^{58,59}$ Furthermore, in BT2, the angle between two adjacent pyridyl rings that belonged to the same triangle $\left(\angle\left(\mathrm{N}_{\mathrm{Py}}-\mathrm{center}-\right.\right.$ $\left.\mathrm{N}_{\mathrm{Py}}\right)_{\text {int }}$ ) expanded to $95^{\circ}\left(97^{\circ}\right.$ in $\mathbf{B T 2}$ '), while the angle between two adjacent pyridyl rings that were not part to the same triangle $\left(\angle\left(\mathrm{N}_{\mathrm{Py}}-\text { center- } \mathrm{N}_{\mathrm{Py}}\right)_{\mathrm{ext}}\right)$ contracted to $85^{\circ}\left(83^{\circ}\right.$ in $\left.\mathbf{B T 2} 2^{\prime}\right)$. Thus, in order to accommodate the long BPDC linker in the triangles of bow tie structures, these angles deviated by $3-5^{\circ}$ from the ideal $\sim 90^{\circ}$ angle between two adjacent pyridyl rings of free $\mathrm{M}^{\prime} \mathrm{TPP}$. The dihedral angles between the core porphyrin plane (pyrrole rings) and pyridyl arms were $76^{\circ}$ in $\mathbf{B T 2}\left(73^{\circ}\right.$ in $\mathbf{B T 2}$ ) and the torsion 
angle between the two phenyl rings of BPDC linker was $42^{\circ}$ in BT2 $\left(37^{\circ}\right.$ in BT2'). Due to the twisted nature of the BPDC core, it was not possible to measure the dihedral angle between the pyrrole ring of M'TPP and BPDC linker located across the bay in the same triangle, but obviously they were not coplanar. The distance from the enclosed $\mathrm{H}_{\mathrm{c}^{\prime}}$ pyrrole protons of $\mathrm{M}^{\prime}$ TPP ligand located inside triangles to the center of the nearest phenyl ring of BPDC linker $\left(d_{\mathrm{Hc}^{\prime}-\mathrm{XDC}}\right)$ was ca. $3.4 \AA$ in BT2 (3.1 $\AA$ in BT2'), which was the longest among the bow tie complexes made of three aromatic XDC linkers employed here. In addition, the distance from the enclosed $\mathrm{H}_{\mathrm{c}^{\prime}}$ pyrrole protons of $\mathrm{M}^{\prime} \mathrm{TPP}$ ligand to the center of the nearest pyridyl ring

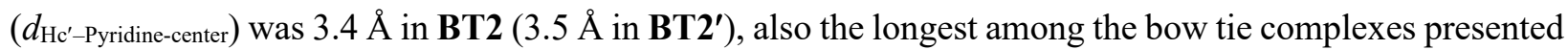
here. As a result, the enclosed $\mathrm{H}_{\mathrm{c}^{\prime}}$ pyrrole protons of BT2 and BT2' were much less shielded and less upfield shifted than those of BDC- and NDC-based bow tie complexes (Figure 6). On the other hand, the long BPDC linked pushed the pyridyl rings of M'TPP closer to the exposed $\mathrm{H}_{\mathrm{c}^{\prime \prime}}$ pyrrole protons $\left(d_{\mathrm{Hc}^{\prime \prime}-\mathrm{Pyridine}-\mathrm{center}}\right.$ $\sim 3.1 \AA$ ) causing a modest shielding and up-field chemical shift of these protons with respect to the $\mathrm{H}_{\mathrm{c}}$ signal of free M'TPP ligands. None of the other bow tie complexes having shorter XDC ligands displayed an upfield NMR chemical shift of the exposed $\mathrm{H}_{\mathrm{c}^{\prime \prime}}$ pyrrole protons; they all shifted downfield from the $\mathrm{H}_{\mathrm{c}}$ peak of free M'TPP because the shorter linkers pulled the adjacent pyridyl rings away from the exposed $\mathrm{H}_{\mathrm{c}^{\prime \prime}}$ pyrrole protons and closer to the enclosed $\mathrm{H}_{\mathbf{c}^{\prime}}$ protons (Figures 3, S2). Furthermore, the crystal structures of BT2 and BT2' also explained why the enclosed $\mathrm{H}_{\mathrm{c}^{\prime}}$ pyrrole protons located inside the triangles were ROESY-coupled with the $\mathrm{H}_{\mathrm{u}}$ and $\mathrm{H}_{\mathrm{v}}$ protons of the adjacent BPDC linkers located across the bay, but the exposed $\mathrm{H}_{\mathrm{c}^{\prime \prime}}$ pyrrole protons were not. Thus, the well-resolved structures of BT2 and BT2' bow tie complexes provided a crystal-clear understanding of their NMR spectra.

Although the entire single-crystal structures of BT2 and BT2' complexes, including their ancillary $\mathrm{Et}_{3} \mathrm{P}$ ligands on the $\mathrm{Pt}(\mathrm{II})$ corners, were fully resolved, only the basic bow tie skeletons of BT3, BT3', and BT4 complexes consisting of the M'TPP core, two parallel BDC and NDC linkers, and four Pt(II) corners were fully resolved, but the fluxional $\mathrm{CH}_{3} \mathrm{CH}_{2}$-groups of ancillary $\mathrm{Et}_{3} \mathrm{P}$ ligands and $\mathrm{TfO}^{-}$anions were not, which caused large $R$-values of the latter. Nevertheless, since the ancillary $\mathrm{Et}_{3} \mathrm{P}$ ligands and counterions were not the integral parts of bow tie structures, the poor resolution of these highly disordered components had little effect on their key structural features that were fully consistent with their respective NMR spectra.

The BDC-based bow tie complexes BT3 and BT3' (Figure 6: $\left[\left\{\left(\mathrm{Et}_{3} \mathrm{P}\right)_{2} \mathrm{Pt}\right\}_{4}\left(\mathrm{M}^{\prime} \mathrm{TPP}\right)(\mathrm{BDC})_{2}\right]^{4+}, \mathrm{M}^{\prime}$ $=\mathrm{Zn}$ or $\mathrm{H}_{2}$ ) also featured a M'TPP core and two parallel BDC linkers held together by four heteroleptic $\left(\mathrm{Et}_{3} \mathrm{P}\right)_{2} \mathrm{Pt}(\mathrm{N}, \mathrm{O})$ corners and crystallized in $C 2 / \mathrm{c}$ and $I 4_{1} / \mathrm{a}$ space groups, respectively. While these two complexes shared the main structural parameters, the ancillary $\mathrm{Et}_{3} \mathrm{P}$ ligands and counterions were less 
disordered in BT3' than in BT3. The $\angle \mathrm{N}-\mathrm{Pt}-\mathrm{O}$ angles at distorted square-planar Pt(II) corners ranged between ca. $78-83^{\circ}$ (two diagonally opposite angles were the same). The distances between two adjacent $\mathrm{Pt}(\mathrm{II})$ corners bridged by a short BDC linker $\left(l_{\mathrm{BDC}}=6.9 \AA\right)^{57}$ were ca. $11.3 \AA\left(d_{\mathrm{Pt}-\mathrm{Pt} / \text { int }}\right)$, whereas the distances between two adjacent Pt(II) corners not bridged by a BDC linker $\left(d_{\mathrm{Pt}-\mathrm{Pt} / \mathrm{ext}}\right)$ were ca. $15.5 \AA$, suggesting that the BDC linkers pulled the two bridged Pt(II) corners closer to each other, which in turn increased the distances between the two non-bridged Pt(II) corners. Consequently, the $\mathrm{N}$-atoms of two adjacent pyridyl rings coordinated to two bridged $\mathrm{Pt}(\mathrm{II})$ corners, i.e., the pyridyl-N atoms that belonged to the same triangle, also came closer to each other $\left(d_{\mathrm{N}-\mathrm{N} / \text { int }} \approx 10.1 \AA\right)$ while the distance between two pyridyl-N atoms that were not part of the same triangle increased $\left(d_{\mathrm{N}-\mathrm{N} / \mathrm{ext}} \approx 11.5 \AA\right)$ with respect to an almost uniform $\mathrm{N}-\mathrm{N}$ distances observed in free M'TPP $\left(d_{\mathrm{N}-\mathrm{N} / \text { free }} \approx 10.9 \AA\right) .{ }^{58,59}$ Thus, even though BDC was much shorter than $d_{\mathrm{N}-\mathrm{N}}$ in free M'TPP, they still formed bow tie complexes instead of prisms via heteroleptic coordination with the $\left(\mathrm{Et}_{3} \mathrm{P}\right)_{2} \mathrm{Pt}^{\mathrm{II}}$ corners. To accommodate the short BDC linkers within the triangles of bow tie-shaped BT3 and BT3' complexes, the angles between two adjacent pyridyl rings coordinated to the bridged $\mathrm{Pt}(\mathrm{II})$ corners $\left(\angle\left(\mathrm{N}_{\mathrm{Py}}-\text { center- } \mathrm{N}_{\mathrm{Py}}\right)_{\text {int }}\right)$ shrunk to $\sim 82-83^{\circ}$, while the angles between two adjacent pyridyl rings not belonging to the same triangle $\left(\angle\left(\mathrm{N}_{\mathrm{Py}}-\text { center- }-\mathrm{N}_{\mathrm{Py}}\right)_{\mathrm{ext}}\right)$ expanded to $\sim 97-98^{\circ}$, i.e., these angles deviated by $7-8^{\circ}$ from the ideal $\sim 90^{\circ}$ angle in free $\mathrm{M}^{\prime}$ TPP. The dihedral angles between the core porphyrin plane (pyrrole rings) and pyridyl arms varied between $76-88^{\circ}$. The dihedral angles between the enclosed pyrrole rings of M'TPP and the BDC core located across the bay of the same triangle were $80-85^{\circ}$, i.e., they were almost orthogonal to each other. The enclosed $\mathrm{H}_{\mathrm{c}^{\prime}}$ pyrrole protons located inside the triangles were projected toward the center of the $\mathrm{BDC}$ ring $\left(d_{\mathrm{Hc}^{\prime}-\mathrm{XDC}} \approx 3.1 \AA\right)$ as well as the adjacent pyridyl rings which were pulled closer $\left(d_{\mathrm{Hc}^{\prime}-\text {-Pyridine-center }} \approx 3 \AA\right)$ by the short BDC linker. As a result, the $\mathrm{H}_{\mathrm{c}^{\prime}}$ protons in BT3 and BT3' complexes were highly shielded and displayed large up-field NMR chemical shifts (Figures 3, S2). On the other hand, since the pyridyl rings of M'TPP moved away from the exposed $\mathrm{H}_{\mathrm{c}^{\prime \prime}}$ pyrrole protons $\left(d_{\mathrm{Hc}^{\prime \prime}-\mathrm{Pyridine}-}\right.$ center $\approx 3.4 \AA$ ), they became less shielded and shifted noticeably downfield from the $\mathrm{H}_{\mathrm{c}}$ signal of free M'TPP ligands. Furthermore, the crystal structures of these bow tie complexes provided valuable insights into why the enclosed $\mathrm{H}_{\mathrm{c}^{\prime}}$ pyrrole protons were ROE-coupled with the $\mathrm{H}_{\mathrm{w}}$ protons of BDC linker located across the bay, but the distant exposed $\mathrm{H}_{\mathrm{c}^{\prime \prime}}$ pyrrole protons were not.

The NDC-based bow tie complex BT4 $\left[\left\{\left(\mathrm{Et}_{3} \mathrm{P}\right)_{2} \mathrm{Pt}\right\}_{4}(\mathrm{ZnTPP})(\mathrm{NDC})_{2}\right]^{4+}$ (Figure 6) was composed of a ZnTPP core and two parallel NDC linkers held together by four heteroleptic $\left(\mathrm{Et}_{3} \mathrm{P}\right)_{2} \mathrm{Pt}(\mathrm{N}, \mathrm{O})$ corners and crystallized in $P 2_{1} / \mathrm{c}$ space group. All four $\angle \mathrm{N}-\mathrm{Pt}-\mathrm{O}$ angles of distorted square-planar $\mathrm{Pt}(\mathrm{II})$ corners were ca. $82^{\circ}$. The distances between two adjacent Pt(II) corners bridged by a NDC linker $\left(d_{\mathrm{Pt}-\mathrm{Pt} / \text { int }}=12.9\right.$ $\AA)^{57}$ were slightly shorter than the distances between two adjacent Pt(II) corners not bridged by a NDC 
linker $\left(d_{\mathrm{Pt}-\mathrm{Pt} / \mathrm{ext}}=14.4 \AA\right)$. The distance between the $\mathrm{N}$-atoms of two adjacent pyridyl rings coordinated to two bridged Pt(II) corners $\left(d_{\mathrm{N}-\mathrm{N} / \text { int }}\right)$ and the distance between two adjacent pyridyl-N atoms that were not part of the same triangle $\left(d_{\mathrm{N}-\mathrm{N} / \mathrm{ext}}\right)$ were almost the same (ca. $10.8 \AA$ ) and close to the uniform distances between two adjacent pyridyl-N atoms of free ZnTPP ligand $\left(d_{\mathrm{N}-\mathrm{N} / \text { free }} \sim 10.9 \AA\right)$. Similarly, the angles between two adjacent pyridyl rings belonging to the same triangle $\left(\angle\left(\mathrm{N}_{\mathrm{Py}}-\mathrm{Center}-\mathrm{N}_{\mathrm{Py}}\right)_{\text {int }}\right)$ and the angles between two adjacent pyridyl rings not belonging to the same triangle $\left(\angle\left(\mathrm{N}_{\mathrm{Py}}-\mathrm{Center}-\mathrm{N}_{\mathrm{Py}}\right)_{\text {ext }}\right)$ were $88^{\circ}$ and $89^{\circ}$, respectively, which were close to $\sim 90^{\circ}$ angle between adjacent pyridyl rings found in free ZnTPP. Thus, the intermediate length of NDC $\left(l_{\mathrm{NDC}}=9.2 \AA\right)$ caused the least distortion of the ZnTPP ligand in BT4 than any other XDC linkers employed here. The dihedral angles between the porphyrin core and pyridyl rings were between $65-76^{\circ}$. The ZnTPP and the NDC planes were oriented nearly orthogonal to each other forming dihedral angles of $87^{\circ}$. The enclosed $\mathrm{H}_{\mathrm{c}^{\prime}}$ pyrrole protons located inside the triangles were projected toward the NDC core $\left(d_{\mathrm{Hc}^{\prime}-\mathrm{XDC}} \approx 3 \AA\right)$, as well as to the center of the adjacent pyridyl rings $\left(d_{\mathrm{Hc}^{\prime}-\mathrm{Pyridine}-\mathrm{center}}\right.$ $\approx 3.2 \AA$ ). As a result, the enclosed $\mathrm{H}_{\mathrm{c}^{\prime}}$ pyrrole protons of BT4 experienced the maximum shielding effect and displayed a larger up-field NMR chemical shift than any other bow tie complexes presented here (vide supra). On the other hand, the distance between the exposed $\mathrm{H}_{\mathrm{c}^{\prime \prime}}$ pyrrole protons and the adjacent pyridyl rings $\left(d_{\mathrm{Hc}^{\prime \prime}-\text { Pyridine-center }} \approx 3.3 \AA\right.$ ) was greater than that found in BPDC-based BT2 and BT2' (ca. $3 \AA$ ) but smaller than that of BDC-based BT3 and BT3' (ca. $3.4 \AA$ ). As a result, the $\mathrm{H}_{\mathrm{c}^{\prime \prime}}$ protons of BT4 displayed a slight downfield chemical shift from the $\mathrm{H}_{\mathrm{c}}$ signal of free ZnTPP (Figures 3, S2). Furthermore, the crystal structure of BT4 also shed light on why the enclosed $\mathrm{H}_{\mathrm{c}^{\prime}}$ pyrrole protons located inside the triangles were ROE-coupled with the $\mathrm{H}_{\mathrm{x}}, \mathrm{H}_{\mathrm{y}}$, and $\mathrm{H}_{\mathrm{z}}$ protons of the adjacent NDC linker located across the bay, but the exposed $\mathrm{H}_{\mathrm{c}^{\prime \prime}}$ pyrrole protons were not.

Energy Optimized Structures of Bow Tie Complexes. Since the crystal structures of BT1 or BT1' complexes containing flexible HDC linker could not be determined, we calculated their energy minimized structures using Gaussian 09 software (Figure 7). To verify the accuracy of the calculated BT1 structure, we also calculated the energy minimized structures of BDC-based BT3 and BT3' complexes by applying the same level of theory (PM-6), which showed excellent agreement with their actual crystal structures (Table 1). Due to similar lengths of HDC and BDC linkers, the calculated BT1 structure displayed strong similarities with the calculated and experimental SXRD structures of BT3. Like other bow tie complexes, BT1 $\left[\left\{\left(\mathrm{Et}_{3} \mathrm{P}\right)_{2} \mathrm{Pt}\right\}_{4}(\mathrm{ZnTPP})(\mathrm{HDC})_{2}\right]^{4+}$ featured a ZnTPP core and two parallel HDC linkers held together by four heteroleptic $\left(\mathrm{Et}_{3} \mathrm{P}\right)_{2} \mathrm{Pt}(\mathrm{N}, \mathrm{O})$ corners. 


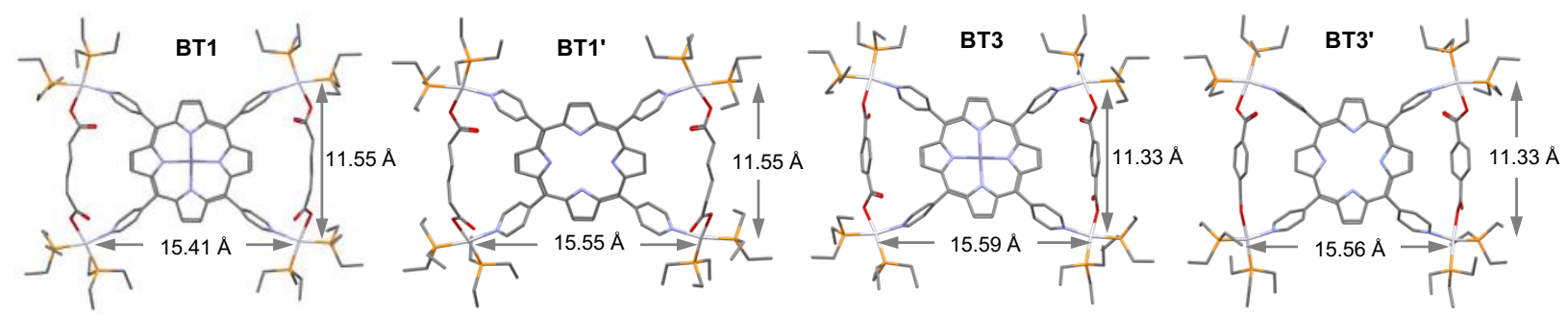

Figure 7. The optimized structures of BT1, BT1', BT3, and BT3' complexes calculated by PM6 method. The distances between adjacent Pt(II) centers bridged and not bridged by XDC linkers $\left(d_{\mathrm{Pt}-\mathrm{Pt} / \text { int }}\right.$ and $\left.d_{\mathrm{Pt}-\mathrm{Pt} / \mathrm{ext}}\right)$ are labeled to show the XDC linkers affected the shapes of the M'TPP ligands.

In the calculated BT1 structure, the $\angle \mathrm{N}-\mathrm{Pt}-\mathrm{O}$ angles at distorted square-planar $\mathrm{Pt}(\mathrm{II})$ corners were ca. 96-98 . The two adjacent HDC-bridged Pt(II) corners $\left(d_{\mathrm{Pt}-\mathrm{Pt} / \mathrm{int}}\right)$ were located ca. $11.6 \AA$ apart, whereas the two adjacent Pt(II) corners not bridged by a HDC linker ( $\left.d_{\text {Pt-Pt/ext }}\right)$ were located ca. $15.4 \AA$ apart. The Pt-Pt distances in BT1 were comparable to those observed in BT3, which suggested that the HDC and BDC linkers have similar lengths. Therefore, like BDC, the short HDC linkers also pulled the two bridged $\mathrm{Pt}(\mathrm{II})$ corners closer to each other, which in turn increased the distances between the two adjacent nonbridged Pt(II) corners. Consequently, the $\mathrm{N}$-atoms of two adjacent pyridyl rings of ZnTPP coordinated to two Pt(II) corners bridged by BDC linkers, i.e., the pyridyl-N atoms that belonged to the same triangle, also came closer to each other $\left(d_{\mathrm{N}-\mathrm{N} / \text { int }} \approx 10.2 \AA\right)$ while the distance between two pyridyl-N atoms that were not part of the same triangle increased $\left(d_{\mathrm{N}-\mathrm{N} / \mathrm{ext}} \approx 11.6 \AA\right)$ from the uniform $\mathrm{N}-\mathrm{N}$ distance (ca. $\left.10.9 \AA\right)$ found in free M'TPP ligands. This happened because in order to accommodate short HDC linkers within the triangles of the bow tie structure, the angles between two adjacent pyridyl rings that belonged to the same triangle $\left(\angle\left(\mathrm{N}_{\mathrm{Py}}-\text { center- } \mathrm{N}_{\mathrm{Py}}\right)_{\text {int }}\right)$ shrunk to $\sim 82^{\circ}$, while the angles between two adjacent pyridyl rings that were not part of the same triangle $\left(\angle\left(\mathrm{N}_{\mathrm{Py}}-\right.\right.$ center- $\left.\mathrm{N}_{\mathrm{Py}}\right)$ ext $)$ expanded to $\sim 98^{\circ}$, i.e., these angles deviated by ca. $8^{\circ}$ from the ideal $\sim 90^{\circ}$ angle found in free $\mathrm{M}^{\prime} \mathrm{TPP}$. The distances from the enclosed $\mathrm{H}_{\mathrm{c}^{\prime}}$ pyrrole protons to the center of nearest pyridyl ring $\left(d_{\mathrm{Hc}^{\prime}-\text { Pyridine-center }}\right)$ was ca. $2.96 \AA$ and to the nearest $\mathrm{H}_{\mathrm{t}}$ protons of HDC linker located across the bay was ca. $2.8 \AA$. The enclosed $\mathrm{H}_{\mathrm{c}^{\prime}}$ protons of HDC-based BT1 were least up-field shifted than those of any other bow tie complexes presented here because they were mostly shielded by the adjacent pyridyl rings while the alkyl chain exerted little shielding effect. On the other hand, since the pyridyl rings of ZnTPP were pulled away from the exposed $\mathrm{H}_{\mathrm{c}^{\prime \prime}}$ pyrrole protons $\left(d_{\mathrm{Hc}^{\prime \prime}-\text { Pyridine-center }} \approx 3.4 \AA\right)$ by short HDC linkers, they became less shielded and shifted downfield from the $\mathrm{H}_{\mathrm{c}}$ signal of free ZnTPP ligand (vide supra). The exposed $\mathrm{H}_{\mathrm{c}^{\prime \prime}}$ pyrrole protons of BT1 and BT3 complexes displayed similar downfield chemical shifts because HDC and BDC linkers having similar lengths pulled the adjacent pyridyl rings away from these protons to the same extent. Furthermore, the calculated BT1 structure provided valuable insights into 
why the enclosed $\mathrm{H}_{\mathrm{c}^{\prime}}$ pyrrole protons were ROE-coupled with the $\mathrm{H}_{\mathrm{t}}$ protons of the adjacent HDC linker located across the bay, but the exposed $\mathrm{H}_{\mathrm{c}^{\prime \prime}}$ protons were not.

Thus, the single-crystal and energy-minimized structures of M'TPP-based tricomponent SCCs further corroborated that regardless of the length (within the range of 6.9-11.2 $\AA$ ) and rigidity of the XDC linkers, the Pt(II)-driven self-assembly of M'TPP and XDC ligands exclusively yielded 2D bow tie complexes instead of 3D prisms. This was possible because the pyridyl arms of M'TPP core coordinated to the XDC-bridged $\mathrm{Pt}(\mathrm{II})$ corners were able to bend to some extent in order to incorporate the XDC linkers having variable lengths within the triangles of bow tie structures. In addition to our comprehensive studies that unequivocally demonstrated bow tie formation with four different XDC linkers, all previously reported M'TPP-based tricomponent SCCs based on other rigid (e.g., isophthalate and BINOL-3,3'dicarboxylate) $)^{33,34}$ and flexible (e.g., heptanedioate and octanedioate) ${ }^{33}$ XDC linkers have also displayed the same characteristic ${ }^{1} \mathrm{H}$ NMR signals of bow tie complexes, i.e., two distinct singlets for the enclosed $\mathrm{H}_{\mathrm{c}^{\prime}}$ and exposed $\mathrm{H}_{\mathrm{c}^{\prime \prime}}$ pyrrole protons of the M'TPP core, although they were thought to be tetragonal prisms. In all those cases, the lengths of the rigid linkers were within the range of XDC linkers employed here that actually formed bow tie complexes, whereas the longer flexible linkers, such as heptanedioate and octanedioate, could bulge out like an arc (i.e., quarter-circles) to form $\infty$-shaped complexes instead of the purported prisms. Therefore, more studies and compelling evidence beyond just ESI-MS data are needed to verify the prism formation by those XDC linkers. Since all XDC linkers employed thus far seemed to have formed M'TPP-based bow tie complexes, we envisioned that much longer and rigid 4,4'-ethyne-1,2diyldibezoate and $N, N$-dicarboxyphenyl naphthalenediimide linkers might form tetragonal prisms by connecting the pyridyl groups of two cofacial M'TPP ligands via heteroligated Pt(II) corners because they are too long to close the triangles of bow tie complexes by intramolecularly bridging two adjacent Pt(II)coordinated pyridyl rings of an M'TPP core. However, these extremely long and rigid linkers neither formed any prisms nor any bow tie complexes, and yielded mixtures of supramolecular assemblies without any well-defined structures. Taken together, these studies suggested that the dihedral angles between the porphyrin core and pyridyl rings of $\mathrm{M}^{\prime} \mathrm{TPP}$ were not suitable for the M'TPP-based 3D prism formation. As a result, M'TPP ligands could only form tricomponent bow tie complexes so long as the XDC linkers could bridge two Pt(II) corners coordinated by two adjacent pyridyl groups of M'TPP forming the bases of isosceles triangles. Beside M'TPP, another tetratopic ligand, 1,2-di(4-pyridyl)ethylene (DPE) was also used previously along with XDC linkers to construct tetragonal prisms. ${ }^{35,36,40}$ Since the dihedral angles and the angles of projection of the pyridyl rings in DPE are different from those of M'TPP, it is plausible that the former indeed formed tetragonal prisms. However, in the light of the discovery of M'TPP-based bow tie 
complexes and better understanding of why M'TPP could not form tetragonal prisms with any XDC linkers, additional 2D NMR and X-ray crystallographic studies would be beneficial to verify the structures of other supramolecular prisms.

\section{CONCLUSION}

In summary, we have demonstrated that Pt(II)-driven social self-assembly of a tetratopic M'TPP ligand and four ditopic XDC linkers having different lengths (6.9-11.2 $\AA$ ) and rigidities consistently yielded novel 2D bow tie complexes $\left[\left\{\left(\mathrm{Et}_{3} \mathrm{P}\right)_{2} \mathrm{Pt}\right\}_{4}\left(\mathrm{M}^{\prime} \mathrm{TPP}\right)(\mathrm{XDC})_{2}\right] \cdot(4 \mathrm{TfO})$ featuring a M'TPP core and two parallel XDC linkers that were held together by four heteroligated $\mathrm{P}^{\mathrm{II}}(\mathrm{N}, \mathrm{O})$ corners. The ${ }^{31} \mathrm{P}$ NMR spectra of the resulting tricomponent SCCs confirmed the formation of heteroleptic $\mathrm{Pt}^{\mathrm{II}}(\mathrm{N}, \mathrm{O})$ corners bearing one carboxylate and one pyridyl groups, while their ${ }^{1} \mathrm{H}$ and 2D NMR spectra presented the most compelling signs of their bow tie structures by revealing that the four pyrrole rings of M'TPP core were located in two distinct chemical environments. Two opposite pyrrole rings carrying the more shielded $\mathrm{H}_{\mathrm{c}^{\prime}}$ protons were located inside two isosceles triangles formed by two parallel XDC linkers that bridged the $\mathrm{Pt}(\mathrm{II})$ corners coordinated to two adjacent pyridyl groups, while the other two pyrrole rings remained exposed, and their $\mathrm{H}_{\mathrm{c}^{\prime \prime}}$ protons were not shielded by or coupled to XDC linkers. These phenomena not only indicated the formation of bow tie complexes, but also ruled out the formation of M'TPP-based tetragonal prisms, which should have possessed all chemically equivalent pyrrole protons. The bow tie complex formation was further corroborated by ESI-MS analysis, while the most direct and compelling evidence came from single-crystal x-ray structures (and energy minimized structures when the crystal structures were not available) that revealed well-resolved bow tie skeletons consisting of a M'TPP core, two parallel XDC linkers, and four $\mathrm{Pt}(\mathrm{II})$ conners although the ancillary $\mathrm{Et}_{3} \mathrm{P}$ groups and counterions were disordered in some cases. These results demonstrated that in order to accommodate different XDC linkers having different lengths (ca. 6.911.2 A) and rigidity into isosceles triangles of bow tie structures, the pyridyl arms of M'TPP ligands moved accordingly departing from their original angles $\left(\sim 90^{\circ}\right)$ of projections. This led to the formation of bow tie complexes exclusively, which were entropically more favored over prisms. Thus, these comprehensive studies of M'TPP-based tricomponent SCCs not only produced novel bow tie complexes, but also demonstrated that these tetratopic ligands were unable to form tetragonal prisms regardless of the length and rigidity dicarboxylate linkers. While the main focus of our fundamental studies presented here was to determine the accurate structures and compositions of M'TPP-based tricomponent SCCs, in the light of these revelations one could argue that some of the fascinating properties and functions, such as photodynamic cancer therapy that were previously attributed to alleged prisms, ${ }^{30,33,34,39,55,56}$ actually 
belonged to bow tie complexes. Further studies of potential applications of these M'TPP-based bow tie complexes as light-harvesting and energy transduction systems are underway in our laboratory.

\section{ASSOCIATED CONTENT}

\section{Supporting Information}

The Supporting Information is available free of charge on the ACS Publications website at DOI: XXXXX. Experimental details and additional data including Figures S1-S6 (PDF), and CIF files.

\section{Accession Code}

CCDC 2081606-2081610 contain crystallographic data, which can be obtained free of charge via www.ccdc.cam.ac.uk/data_request/cif, or by emailing data_request@ccdc.cam.ac.uk, or by contacting The Cambridge Crystallographic Data Centre, 12 Union Road, Cambridge CB2 1EZ, UK.

\section{AUTHOR INFORMATION}

\section{*Corresponding Author}

Email: souravs@clemson.edu

\section{ORCID}

Sourav Saha: 0000-0001-6610-4820

Paola A. Benavides: 0000-002-1701-5036

M. Andrey Joaqui-Joaqui: 0000-001-7446-6004

\section{Note}

The authors declare no competing financial interest.

\section{ACKNOWLEDGEMENTS}

This work was supported by the National Science Foundation (NSF award nos. CHE-1660329 and DMR1809092) and Clemson University. We also acknowledge the NSF-MRI grant CHE-1725919 for the 500 MHz NMR instrument used in our studies. We thank Dr. Colin McMillen for single-crystal X-ray analysis and Dr. William T. Pennington Jr. for valuable discussions.

\section{REFERENCES}


1. Fujita, M.; Yazaki, J.; Ogura, K. Preparation of a Macrocyclic Polynuclear Complex, [(En)Pd(4,4'Bpy $]_{4}\left(\mathrm{NO}_{3}\right)_{8}(\mathrm{En}=$ Ethylenediamine, Bpy $=$ Bipyridine $)$, Which Recognizes an Organic Molecule in Aqueous Media. J. Am. Chem. Soc. 1990, 112, 5645-5647.

2. Stang, P. J.; Cao, D. H. Transition Metal Based Cationic Molecular Boxes. Self-Assembly of Macrocyclic Platinum(II) and Palladium(II) Tetranuclear Complexes. J. Am. Chem. Soc. 1994, 116, 4981-4982.

3. Stang, P. J.; Olenyuk, B.; Fan, J.; Arif, A. M. Combining Ferrocene and Molecular Squares: SelfAssembly of Heterobimetallic Macrocyclic Squares Incorporating Mixed Transition Metal Systems and a Main Group Element. Single-Crystal X-ray Structure of $\left[\mathrm{Pt}(\mathrm{dppf})\left(\mathrm{H}_{2} \mathrm{O}\right)_{2}\right][\mathrm{OTf}]_{2}$. Organometallics 1996, 15, 904-908.

4. Stang, P. J.; Olenyuk, B. Self-Assembly, Symmetry, and Molecular Architecture: Coordination as the Motif in the Rational Design of Supramolecular Metallacyclic Polygons and Polyhedra. Acc. Chem. Res. 1997, 30, 502-518.

5. Lees, A. J.; Sun, S.-S. Self-Assembly Organometallic Squares with Terpyridyl Metal Complexes as Bridging Ligands. Inorg. Chem. 2001, 40, 3154-3160.

6. Würthner, F.; You, C.-C.; Saha-Möller, C. R. Metallosupramolecular Squares: From Structure to Function. Chem. Soc. Rev. 2004, 33, 133-146.

7. Gianneschi, N. C.; Masar III, M. S.; Mirkin, C. A. Development of a Coordination Chemistry-Based Approach for Functional Supramolecular Structures, Acc. Chem. Res. 2005, 38, 825-837.

8. Northrop, B. H.; Zheng, Y.-R.; Chi, K.-W.; Stang, P. J. Self-Organization in Coordination-Driven Self-Assembly. Acc. Chem. Res. 2009, 42, 1554-1563.

9. $\quad$ Chifotides, H. T.; Giles, I. D.; Dunbar, K. R. Supramolecular Architectures with $\pi$-Acidic 3,6-Bis(2Pyridyl)-1,2,4,5-Tetrazine Cavities: Role of Anion- $\pi$ Interactions in the Remarkable Stability of Fe(II) Metallacycles in Solution. J. Am. Chem. Soc. 2013, 135, 3039-3055.

10. Gordillo, M. A.; Benavides, P. A.; Saha, S. Anion/Naphthalenediimide Interactions in a Pd(II)-Based Tetrameric Metallocycle. Cryst. Growth Des. 2019, 19, 6017-6022.

11. Kumazawa, K.; Biradha, K.; Kusukawa, T.; Okano, T.; Fujita, M. Multicomponent Assembly of a Pyrazine-Pillared Coordination Cage that Selectively Binds Planar Guests by Intercalation. Angew. Chemie Int. Ed. 2003, 42, 3909-3913.

12. Chakrabarty, R.; Mukherjee, P. S.; Stang, P. J. Supramolecular Coordination: Self-Assembly of Finite Two- and Three-Dimensional Ensembles. Chem. Rev. 2011, 111, 6810-6918.

13. Cook T. R.; Zheng, Y.-R.; Stang, P. J. Metal-organic frameworks and self-assembled supramolecular coordination complexes: Comparing and contrasting the design, synthesis, and functionality of metal-organic materials. Chem. Rev. 2013, 112, 734-777.

14. Cook, T. R.; Stang, P. J. Recent Developments in the Preparation and Chemistry of Metallacycles and Metallacages via Coordination. Chem. Rev. 2015, 115, 7001-7045.

15. Zarra, S.; Wood, D. M.; Roberts, D. A.; Nitschke, J. R. Molecular Containers in Complex Chemical Systems. Chem. Soc. Rev. 2015, 44, 419-432.

16. Fujii, S.; Tada, T.; Komoto, Y.; Osuga, T.; Murase, T.; Fujita, M.; Kiguchi, M., Rectifying ElectronTransport Properties through Stacks of Aromatic Molecules Inserted into a Self-Assembled Cage. $J$. Am. Chem. Soc. 2015, 137, 5939-5947.

17. Bloch, W. M.; Clever, G. H. Integrative Self-Sorting of Coordination Cages Based on 'Naked' Metal Ions. Chem. Commun. 2017, 53, 8506-8516. 
18. Ronson, T. K.; Meng, W.; Nitschke, J. R. Design Principles for the Optimization of Guest Binding in Aromatic-Paneled Fe ${ }_{4}^{\mathrm{II}} \mathrm{L}_{6}$ Cages. J. Am. Chem. Soc., 2017, 139, 9698-9707.

19. Brenner, W.; Ronson, T. K.; Nitschke, J. R. Separation and Selective Formation of Fullerene Adducts within an $\mathrm{M}_{8}^{\mathrm{II}} \mathrm{L}_{6}$ Cage. J. Am. Chem. Soc., 2017, 139, 75-78.

20. Saha, S.; Regeni, I.; Clever, G. H. Structure Relationships between Bis-Monodentate Ligands and Coordination Driven Self-Assemblies. Coord. Chem. Rev. 2018, 374, 1-14.

21. Pullen, S.; Clever, G. H. Mixed-Ligand Metal-Organic Frameworks and Heteroleptic Coordination Cages as Multifunctional Scaffolds-A Comparison. Acc. Chem. Res. 2018, 51, 3052-3064.

22. Chakraborty, S.; Newkome, G. R. Terpyridine-Based Metallosupramolecular Constructs: Tailored Monomers to Precise 2D-Motifs and 3D-Metallocages. Chem. Soc. Rev. 2018, 47, 3991-4016.

23. Bardhan, D.; Chand, D. K. Palladium(II)-Based Self-Assembled Heteroleptic Coordination Architectures: A Growing Family. Chem. Eur. J. 2019, 25, 12241-12269.

24. Kitagawa, S.; Kitaura, R.; Noro, S. Functional Porous Coordination Polymers. Angew. Chem. Int. Ed. 2004, 43, 2334-2375.

25. Leong, W. L.; Vittal, J. J. One-Dimensional Coordination Polymers: Complexity and Diversity in Structures, Properties, and Applications. Chem. Rev. 2011, 111, 688-764.

26. Furukawa, H.; Cordova, K. E.; O'Keeffe, M.; Yaghi, O. M. The Chemistry and Applications of Metal-Organic Frameworks. Science 2013, 341, 974-986.

27. Chi, K. W.; Addicott, C.; Arif, A. M.; Stang, P. J. Ambidentate Pyridyl-Carboxylate Ligands in the Coordination-Driven Self-Assembly of 2D Pt Macrocycles: Self-Selection for a Single Isomer. $J$. Am. Chem. Soc. 2004, 126, 16569-16574.

28. Wang, M.; Zheng, Y.-R.; Ghosh, K.; Stang, P. J. Metallosupramolecular Tetragonal Prisms via Multicomponent Coordination-Driven Self-Assembly. J. Am. Chem. Soc. 2010, 132, 6282-6283.

29. Zheng, Y. R.; Lan, W. J.; Wang, M.; Cook, T. R.; Stang, P. J. Designed Post-Self-Assembly Structural and Functional Modifications of a Truncated Tetrahedron. J. Am. Chem. Soc. 2011, 133, $17045-17055$.

30. Zheng, Y.-R.; Zhao, Z.; Wang, M.; Ghosh, K.; Pollock, J. B.; Cook, T. R.; Stang, P. J. A Facile Approach toward Multicomponent Supramolecular Structures: Selective Self-Assembly via Charge Separation. J. Am. Chem. Soc. 2010, 132, 16873-16882.

31. Wang, M.; Zheng, Y.-R.; Cook, T. R.; Stang, P. J. Construction of Functionalized Metallosupramolecular Tetragonal Prisms via Multicomponent Coordination-Driven Self-Assembly. Inorg. Chem. 2011, 50, 6107-6113.

32. Pollock, J. B.; Cook, T. R.; Schneider, G. L.; Stang, P. J. Multi-Component Coordination-Driven Self-Assembly: Construction of Alkyl-Based Structures and Molecular Modelling. Chem. Asian J. 2013, 8, 2423-2429.

33. Shi, Y.; Sánchez-Molina, I.; Cao, C.; Cook, T. R.; Stang, P. J. Synthesis and Photophysical Studies of Self-Assembled Multicomponent Supramolecular Coordination Prisms Bearing Porphyrin Faces. Proc. Natl. Acad. Sci. USA 2014, 111, 9390-9395.

34. Ye, Y.; Cook, T. R.; Wang, S. P.; Wu, J.; Li, S.; Stang, P. J. Self-Assembly of Chiral Metallacycles and Metallacages from a Directionally Adaptable BINOL-Derived Donor. J. Am. Chem. Soc. 2015, 137, 11896-11899.

35. Yan, X.; Cook, T. R.; Wang, P.; Huang, F.; Stang, P. J. Highly emissive platinum(II) metallacages. Nature Chem. 2015, 7, 342-348. 
36. Yu, G.; Cook, T. R.; Li, Y.; Yan, X.; Wu, D.; Shao, L.; Shen, J.; Tang, G.; Huang, F.; Chen, X.; Stang, P. J. Tetraphenylethene-Based Highly Emissive Metallacage as a Component of Theranostic Supramolecular Nanoparticles. Proc. Natl. Acad. Sci. 2016, 113, 13720-13725.

37. Li, Z.; Yan, X.; Huang, F.; Sepehrpour, H.; Stang, P. J. Near-Infrared Emissive Discrete Platinum(II) Metallacycles: Synthesis and Application in Ammonia Detection. Org. Lett. 2017, 19, 5728-5731.

38. Zhang, M.; Saha, M. L.; Wang, M.; Zhou, Z.; Song, B.; Lu, C.; Yan, X.; Li, X.; Huang, F.; Yin, S.; Stang, P. J. Multicomponent Platinum(II) Cages with Tunable Emission and Amino Acid Sensing. J. Am. Chem. Soc. 2017, 139, 5067-5074.

39. Yu, G.; Yu, S.; Saha, M. L.; Zhou, J.; Cook, T. R.; Yung, B. C.; Chen, J.; Mao, Z.; Zhang, F.; Zhou, Z.; Liu, Y.; Shao, L.; Wang, S.; Gao, C.; Huang, F.; Stang, P. J.; Chen, X. A Discrete Organoplatinum(II) Metallacage as a Multimodality Theranostic Platform for Cancer Photochemotherapy. Nat. Commun. 2018, 9, 1-18.

40. Sun, Y.; Yao, Y.; Wang, H.; Fu, W.; Chen, C.; Saha, M. L.; Zhang, M.; Datta, S.; Zhou, Z.; Yu, H.; Li, X.; Stang, P. J. Self-Assembly of Metallacages into Multidimensional Suprastructures with Tunable Emissions. J. Am. Chem. Soc. 2018, 140, 12819-12828.

41. Chang, X.; Zhou, Z.; Shang, C.; Wang, G.; Wang, Z.; Qi, Y.; Li, Z.-Y.; Wang, H.; Cao, L.; Li, X.; Fang, Y.; Stang, P. J. Coordination-Driven Self-Assembled Metallacycles Incorporating Pyrene: Fluorescence Mutability, Tunability, and Aromatic Amine Sensing. J. Am. Chem. Soc. 2019, 141, $1757-1765$.

42. Chang, X.; Zhou, Z.; Shang, C.; Wang, G.; Wang, Z.; Qi, Y.; Li, Z. Y.; Wang, H.; Cao, L.; Li, X.; Fang, Y.; Stang, P. J. Coordination-Driven Self-Assembled Metallacycles Incorporating Pyrene: Fluorescence Mutability, Tunability, and Aromatic Amine Sensing. J. Am. Chem. Soc. 2019, 141, $1757-1765$.

43. Sun, Y.; Chen, C.; Liu, J.; Stang, P. J. Recent Developments in the Construction and Applications of Platinum-Based Metallacycles and Metallacages Via Coordination. Chem. Soc. Rev. 2020, 49, 38893919.

44. Ghosh, S.; Mukherjee, P. S. Self-Assembled Pd(II) Metallocycles Using an Ambidentate Donor and the Study of Square-Triangle Equilibria. Inorg. Chem. 2009, 48, 2605-2613.

45. Bar, A. K.; Mostafa, G.; Mukherjee, P. S. A Pd 6 Molecular Cage via Multicomponent Self-Assembly Incorporating Both Neutral and Anionic Linkers. Inorg. Chem. 2010, 49, 7647-7649.

46. Samanta, D.; Shanmugaraju, S.; Joshi, S. A.; Patil, Y. P.; Nethaji, M.; Mukherjee, P. S. Pillar height dependent formation of unprecedented $\mathrm{Pd}_{8}$ molecular swing and $\mathrm{Pd}_{6}$ molecular boat via multicomponent self-assembly. Chem. Commun. 2012, 48, 2298-2300.

47. García-Simón, C.; Garcia-Borràs, M.; Gómez, L.; Garcia-Bosch, I.; Osuna, S.; Swart, M.; Luis, J. M.; Rovira, C.; Almedia, M.; Imaz, I.; Maspoch, D.; Costas, M.; Ribas, X. Self-Assembled Tetragonal Prismatic Molecular Cage Highly Selective for Anionic $\pi$ Guests. Chem. Eur. J. 2013, 19, $1445-1456$.

48. García-Simón, C.; Garcia-Borràs, M.; Gómez, L.; Parella, T.; Osuna, S.; Juanhuix, J.; Imaz, I.; Maspoch, D.; Costas, M.; Ribas, X. Sponge-like Molecular Cage for Purification of Fullerenes. Nat. Commun. 2014, 5, 5557.

49. Colomban, C.; Szalóki, G.; Allain, M.; Gómez, L.; Goeb, S.; Sallé, M.; Costas, M.; Ribas, X. Reversible $\mathrm{C}_{60}$ Ejection from a Metallocage through the Redox-Dependent Binding of a Competitive Guest. Chem. Eur. J. 2017, 23, 3016-3022. 
50. Colomban, C.; Martin-Diaconescu, V.; Parella, T.; Goeb, S.; García-Simón, C.; Lloret-Fillol, J.; Costas, M.; Ribas, X. Design of Zn-, Cu-, and Fe-Coordination Complexes Confined in a SelfAssembled Nanocage. Inorg. Chem. 2018, 57, 3529-3539.

51. Fuertes-Espinosa, C.; Gómez-Torres, A.; Morales-Martínez, R.; Rodríguez-Fortea, A.; GarcíaSimón, C.; Gándara, F.; Imaz, I.; Juanhuix, J.; Maspoch, D.; Poblet, J. M.; Echegoyen, L.; Ribas, X. Purification of Uranium-Based Endohedral Metallofullerenes (EMFs) by Selective Supramolecular Encapsulation and Release. Angew. Chem. Int. Ed. 2018, 57, 11294-11299.

52. Colomban, C.; Fuertes-Espinosa, C.; Goeb, S.; Sallé, M.; Costas, M.; Blancafort, L.; Ribas, X. SelfAssembled Cofacial Zinc-Porphyrin Supramolecular Nanocapsules as Tunable ${ }^{1} \mathrm{O}_{2}$ Photosensitizers. Chem. Eur. J. 2018, 24, 4371-4381.

53. García-Simón, C.; Monferrer, A.; Garcia-Borràs, M.; Imaz, I.; Maspoch, D.; Costas, M.; Ribas, X. Sponge-like Molecular Cage for Purification of Fullerenes. Chem. Commun. 2019, 55, 798-801.

54. Ubasart, E.; Borodin, O.; Fuertes-Espinosa, C.; Xu, Y.; García-Simón, C.; Gómez, L.; Juanhuix, J.; Gándara, F.; Imaz, I.; Maspoch, D.; Delius, M.; Ribas, X. A Three-Shell Supramolecular Complex Enables the Symmetry-Mismatched Chemo- and Regioselective Bis-Functionalization of $\mathrm{C}_{60}$. Nat. Chem. 2021, DOI: 10.1038/s41557-021-00658-6.

55. Yu, G.; Ye, Y.; Tong, Z.; Yang, J.; Li, Z.; Hua, B.; Shao, L.; Li, S. A Porphyrin-Based Discrete Tetragonal Prismatic Cage: Host-Guest Complexation and Its Application in Tuning LiquidCrystalline Behavior. Macromol. Rapid Commun. 2016, 37, 1540-1547.

56. Zhao, Z.; Zhang, Z.; Wang, H.; Li, X.; Zhang, M. Multicomponent Porphyrin-Based Tetragonal Prismatic Metallacages and Their Photophysical Properties. Isr. J. Chem. 2019, 59, 299-305.

57. Eddaoudi, M.; Kim, J.; Rosi, N.; Vodak, D.; Wachter, J.; O’Keeffe, M.; Yaghi, M. Systematic Design of Pore Size and Functionality in Isoreticular MOFs and Their Application in Methane Storage. Science 2002, 295, 469-472.

58. Lipstman, S.; Goldberg, I. Hydrogen-Bonded Three-Dimensional Network of a Lanthanum(III) Exocyclic Complex with 5,10,15,20-Tetra-4-Pyridyl-Porphyrin. Acta Cryst. Sect. C. 2009, 65, 371373.

59. Dinelli, L. R.; Von Poelhsitz, G.; Castellano, E. E.; Ellena, J.; Galembeck, S. E.; Batista, A. A. On an Electrode Modified by a Supramolecular Ruthenium Mixed Valence ( $\left.\mathrm{Ru}^{\mathrm{II}} / \mathrm{Ru}^{\mathrm{III}}\right)$ DiphosphinePorphyrin Assembly. Inorg. Chem. 2009, 48, 4692-4700. 
$\underline{\text { Table of Contents }}$

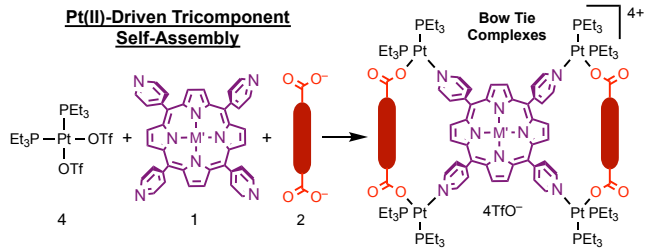




\section{Supporting Information}

\section{Pt(II)-Coordinated Tricomponent Supramolecular Assemblies of Tetrapyridyl Porphyrin and}

Dicarboxylate Ligands: Are They 2D Bow Ties or 3D Prisms?

Paola A. Benavides, ${ }^{\dagger}$ Monica A. Gordillo, ${ }^{\dagger}$ Ashok Yadav, ${ }^{\dagger}$ M. Andrey Joaqui-Joaqui, ${ }^{\dagger}$ and Sourav Saha*, ${ }^{\dagger}$ ${ }^{\dagger}$ Department of Chemistry, Clemson University, Clemson, South Carolina 29634, United States

*Department of Chemistry, University of Minnesota, Minneapolis, MN 55455, United States

*Email: souravs@clemson.edu

\section{$\underline{\text { Table of Contents }}$}

General Materials and Methods

Synthesis and Characterization of Bow Tie Complexes

Figure S1. The ${ }^{31} \mathrm{P}$ NMR spectra of $c i s-\left(\mathrm{Et}_{3} \mathrm{P}\right)_{2} \mathrm{Pt}(\mathrm{TfO})$ and BT1-BT4' complexes

Figure S2. The partial ${ }^{1} \mathrm{H}$ NMR spectra free $\mathrm{H}_{2} \mathrm{TPP}$ and BT1'-BT4' complexes

Figure S3. The entire ${ }^{1} \mathrm{H}$ NMR spectra of BT1-BT4 and BT1'-BT4' complexes

Figure S4. The ${ }^{1} \mathrm{H}-{ }^{1} \mathrm{H}$ COSY NMR spectra of BT1-BT4 and BT1'-BT4' complexes

Figure S5. The ${ }^{1} \mathrm{H}-{ }^{1} \mathrm{H}$ ROESY NMR spectra of BT1'-BT4' complexes

Figure S6. The ESI-MS data of bow tie complexes 


\section{General Materials and Methods}

Reagents, starting materials, and solvents were purchased from Sigma-Aldrich, Acros Organic and TCI America. cis- $\mathrm{Pt}\left(\mathrm{PEt}_{3}\right)_{2}(\mathrm{OTf})_{2}$ and $\mathrm{ZnTPP}$ were prepared following reported procedures. ${ }^{1,2}$

The ${ }^{1} \mathrm{H}$, COSY, and ROESY NMR spectra were recorded on a Bruker NEO $500 \mathrm{MHz}$ NMR spectrometers. For ROESY, a mixing time of $491 \mathrm{~ms}$ was employed for all samples. The ${ }^{31} \mathrm{P}$ NMR spectra was recorded at $122 \mathrm{MHz}$ using the same instrument.

Electrospray ionization mass spectra (ESIMS) of the M'TPP-based tricomponent bow tie complexes were recorded on a Bruker BioTOF II ESI/TOF-MS instrument at the Waters Center for Innovation in Mass Spectrometry of the Department of Chemistry at the University of Minnesota, Twin Cities.

The single crystal X-ray diffraction (SXRD) data were collected on a Bruker D8 Venture dual source diffractometer equipped with $\mathrm{Cu}$ and Mo radiation sources and CMOS detector. The crystal structures of bow tie complexes were solved and refined by using Bruker SHELXTL software package. The crystals of some bow tie complexes diffracted poorly, and the ethyl groups on $\mathrm{Et}_{3} \mathrm{P}$ ligands were highly disordered due to thermal motions. These disordered ethyl groups were fixed by using the DFIX, DANG commands. In addition, the coordination of bridging XDC linkers with two Pt(II) corners also created strain and made them slightly bend and disordered in BT3, BT3', and BT4. AFIX and DFIX commands were applied to fix these issues. The highly disordered triflate anions were hard to assign and omitted by PLATON/SQUEEZE in some cases, while in other cases they were assigned fully/partially using SAME/SIMU commands. ISOR and RIGU commands were applied for the atoms that were not behaving well isothermally. A SHEL cutoff was applied to remove the weak reflections in most of the structures. The disordered solvent molecules were also removed by PLATON/SQUEEZE model. The significant amount of disorder and weak diffraction of some crystals resulted in several A- and B-alerts, which have been addressed properly in the corresponding checkCIFs, while the commands applied to fix the disordered atoms and groups caused large R-values in those cases. Nevertheless, the basic bow tie skeletons of all complexes were well resolved and the disordered $\mathrm{Et}_{3} \mathrm{P}$ groups, counter anions, and solvent molecules had practically no impact on the overall shape and geometry of these complexes.

The geometry optimized structures were calculated with semi-empirical methods using PM6 model on Gaussian software version 09.

\section{Synthesis and Characterization}

Dicarboxylate (XDC) Ligands. In general, aqueous solutions of 2.5 equiv. of $\mathrm{KOH}$ was added to respective dicarboxylic acids (i.e., hexane-, 4,4'-biphenyl, 1,4-benzene-, and 2,6-naphthalene dicarboxylic acids) and the resulting mixtures were stirred until the solids were fully solubilized due to salt formation. At this point, $\mathrm{MeOH}$ was added to the solution mixtures, which led to the precipitation of dipotassium dicarboxylate salts. The resulting solids were filtrated, washed thoroughly with cold $\mathrm{MeOH}$, and recrystallized from $\mathrm{MeOH}$, to remove excess of $\mathrm{KOH}$ and obtain pure products.

General Protocol of Tricomponent Self-Assembly of cis-Pt(Et $\left.{ }_{3} P\right)_{2}(\mathrm{TfO})_{2}$, M'TPP, and XDC. To a mixture of $c i s-\mathrm{Pt}\left(\mathrm{PEt}_{3}\right)_{2}(\mathrm{OTf})_{2}$ (4 equiv.), $\mathrm{M}^{\prime} \mathrm{TPP}\left(\mathrm{M}^{\prime}=\mathrm{Zn}\right.$ or $\mathrm{H}_{2}, 1$ equiv.), and XDC (XDC = HDC, BPDC, $\mathrm{BDC}$, and NDC, 2 equiv.) placed in a $20 \mathrm{~mL}$ screw-capped glass was added a 1:1:1 $\mathrm{CH}_{2} \mathrm{Cl}_{2} / \mathrm{MeNO}_{2} / \mathrm{MeCN}$ 
solvent mixture $(5 \mathrm{~mL})$. The reaction mixtures were then stirred at $60{ }^{\circ} \mathrm{C}$ for overnight. After allowing the reaction mixtures to cool down to room temperature, they were filtered to remove insoluble parts. $\mathrm{Et}_{2} \mathrm{O}$ was added to the filtrates and the resulting purple precipitates were collected by centrifugation. This washing process was repeated three times to obtain pure tricomponent bow tie complexes BT1-BT4 containing ZnTPP and BT1'-BT4' containing $\mathrm{H}_{2}$ TPP cores.

BT1. cis-Pt(PEt $)_{2}(\mathrm{OTf})_{2}(4.49 \mathrm{mg}, 0.0059 \mathrm{mmol}), \mathrm{ZnTPP}(1 \mathrm{mg}, 0.00147 \mathrm{mmol})$, and HDC (0.64 mg, $0.0029 \mathrm{mmol})$ in a $1: 1: 1 \mathrm{DCM} / \mathrm{MeNO}_{2} / \mathrm{MeCN}$ solvent mixture $(1 \mathrm{~mL}) .{ }^{1} \mathrm{H}$ NMR $\left(500 \mathrm{MHz}\right.$, acetone- $\left.d_{6}\right) \delta$ $=9.28\left(\mathrm{br} \mathrm{s}, 8 \mathrm{H}, \mathrm{H}_{\mathrm{a}}\right), 9.18\left(\mathrm{~s}, 4 \mathrm{H}, \mathrm{H}_{\mathrm{c}^{\prime \prime}}\right), 8.50\left(\mathrm{~d}, J=5.8 \mathrm{~Hz}, 8 \mathrm{H}, \mathrm{H}_{\mathrm{b}}\right), 8.38\left(\mathrm{~s}, 4 \mathrm{H}, \mathrm{H}_{\mathrm{c}^{\prime}}\right), 2.33-2.15(\mathrm{~m}, 56 \mathrm{H}$, $\mathrm{H}_{\mathrm{s}}$ and $\left.\mathrm{H}_{-\mathrm{CH} 2-}\right), 1.65$ (br s, $\left.8 \mathrm{H}, \mathrm{H}_{\mathrm{t}}\right), 1.51-1.36\left(\mathrm{~m}, 72 \mathrm{H}, \mathrm{H}_{-\mathrm{CH} 3}\right) \mathrm{ppm} .{ }^{31} \mathrm{P}$ NMR $\left(202 \mathrm{MHz}\right.$, acetone- $\left.d_{6}\right) \delta 6.23$ $(\mathrm{d}, J=20.5 \mathrm{~Hz}), 0.23(\mathrm{~d}, J=21.0 \mathrm{~Hz})$. ESI-MS $(\mathrm{m} / z)$ Calculated for $\mathrm{C}_{102} \mathrm{H}_{160} \mathrm{~F}_{6} \mathrm{~N}_{8} \mathrm{O}_{14} \mathrm{P}_{8} \mathrm{Pt}_{4} \mathrm{~S}_{2} \mathrm{Zn}[\mathrm{M}-2 \mathrm{TfO}]^{2+}$ : 1497.14. Found: 1497.09.

BT2. cis-Pt( $\left(\mathrm{PEt}_{3}\right)_{2}(\mathrm{OTf})_{2}(14.1 \mathrm{mg}, 0.019 \mathrm{mmol})$, ZnTPP (3.3 mg, $\left.0.0048 \mathrm{mmol}\right)$, and BPDC (3.08 $\mathrm{mg}$, $0.0096 \mathrm{mmol})$ in a $1: 1: 1 \mathrm{DCM} / \mathrm{MeNO}_{2} / \mathrm{MeCN}$ solvent mixture $(5 \mathrm{~mL}) .{ }^{1} \mathrm{H}$ NMR $\left(500 \mathrm{MHz}\right.$, acetone- $\left.d_{6}\right) \delta$ $=9.28\left(\mathrm{br} \mathrm{s}, 8 \mathrm{H}, \mathrm{H}_{\mathrm{a}}\right), 8.58\left(\mathrm{~s}, 4 \mathrm{H}, \mathrm{H}_{\mathrm{c}^{\prime \prime}}\right), 8.38\left(\mathrm{~d}, J=5.6 \mathrm{~Hz}, 8 \mathrm{H}, \mathrm{H}_{\mathrm{b}}\right), 8.10\left(\mathrm{~d}, J=8.1 \mathrm{~Hz}, 8 \mathrm{H}, \mathrm{H}_{\mathrm{u}}\right), 7.98(\mathrm{~d}, J$ $\left.=8.2 \mathrm{~Hz}, 8 \mathrm{H}, \mathrm{H}_{\mathrm{v}}\right), 7.74\left(\mathrm{~s}, 4 \mathrm{H}, \mathrm{H}_{\mathrm{c}^{\prime}}\right), 2.44-2.18\left(\mathrm{~m}, 48 \mathrm{H}, \mathrm{H}_{-\mathrm{CH} 2-}\right), 1.58-1.42\left(\mathrm{~m}, 72 \mathrm{H}, \mathrm{H}_{-\mathrm{CH} 3}\right) .{ }^{31} \mathrm{P}$ NMR $\left(122 \mathrm{MHz}\right.$, acetone- $\left.d_{6}\right) \delta 6.83(\mathrm{~d}, J=20.6 \mathrm{~Hz}), 1.78(\mathrm{~d}, J=21.2 \mathrm{~Hz})$. ESI-MS $(\mathrm{m} / \mathrm{z})$ Calculated for $\mathrm{C}_{118} \mathrm{H}_{160} \mathrm{~F}_{6} \mathrm{~N}_{8} \mathrm{O}_{14} \mathrm{P}_{8} \mathrm{Pt}_{4} \mathrm{~S}_{2} \mathrm{Zn}[\mathrm{M}-2 \mathrm{TfO}]^{2+}$ 1593.23, Found: 1593.58 .

BT3. cis-Pt(PEt $)_{2}(\mathrm{OTf})_{2}(14.1 \mathrm{mg}, 0.019 \mathrm{mmol})$, BDC (2.34 mg, $\left.0.0096 \mathrm{mmol}\right)$ and ZnTPP (3.3 mg, 0.0048 $\mathrm{mmol})$ in a 1:1:1 DCM/MeNO$/ 2 / \mathrm{MeCN}$ solvent mixture $(5 \mathrm{~mL}) .{ }^{1} \mathrm{H}$ NMR $\left(500 \mathrm{MHz}\right.$, acetone- $\left.d_{6}\right) \delta=9.35$ (br s, 8H, $\mathrm{H}_{\mathrm{a}}$ ), $9.16\left(\mathrm{~s}, 4 \mathrm{H}, \mathrm{H}_{\mathrm{c}^{\prime \prime}}\right), 8.36\left(\mathrm{~d}, J=6.3 \mathrm{~Hz}, 8 \mathrm{H}, \mathrm{H}_{\mathrm{b}}\right), 8.09\left(\mathrm{~s}, 8 \mathrm{H}, \mathrm{H}_{\mathrm{w}}\right), 7.13\left(\mathrm{~s}, 4 \mathrm{H}, \mathrm{H}_{\mathrm{c}^{\prime}}\right), 2.40-2.19$ (m, 48H, $\left.\mathrm{H}_{-\mathrm{CH} 2-}\right), 1.57-1.41\left(\mathrm{~m}, 72 \mathrm{H}, \mathrm{H}_{-\mathrm{CH} 3}\right) \mathrm{ppm} .{ }^{31} \mathrm{P}$ NMR $\left(203 \mathrm{MHz}\right.$, acetone- $\left.d_{6}\right) \delta=6.02(\mathrm{~d}, J=20.5$ $\mathrm{Hz}), 1.01(\mathrm{~d}, J=21.4 \mathrm{~Hz}) \mathrm{ppm}$. ESI-MS $(\mathrm{m} / \mathrm{z})$ Calcualted for $\mathrm{C}_{106} \mathrm{H}_{152} \mathrm{~F}_{6} \mathrm{~N}_{8} \mathrm{O}_{14} \mathrm{P}_{8} \mathrm{Pt}_{4} \mathrm{~S}_{2} \mathrm{Zn}[\mathrm{M}-2 \mathrm{TfO}]^{2+}$ : 1517.13. Found: 1517.01.

BT4. cis-Pt(PEt $)_{2}(\mathrm{OTf})_{2}(14.1 \mathrm{mg}, 0.019 \mathrm{mmol}), \mathrm{BDC}$ (2.83 mg, $\left.0.0096 \mathrm{mmol}\right)$ and ZnTPP (3.3 mg, 0.0048 $\mathrm{mmol})$ in a $1: 1: 1 \mathrm{DCM} / \mathrm{MeNO}_{2} / \mathrm{MeCN}$ solvent mixture $(5 \mathrm{~mL}) .{ }^{1} \mathrm{H}$ NMR $\left(500 \mathrm{MHz}\right.$, acetone- $\left.d_{6}\right) \delta=9.37$ (br s, $\left.8 \mathrm{H}, \mathrm{H}_{\mathrm{a}}\right), 8.86\left(\mathrm{~s}, 4 \mathrm{H}, \mathrm{H}_{\mathrm{c}^{\prime \prime}}\right), 8.71\left(\mathrm{~s}, 4 \mathrm{H}, \mathrm{H}_{\mathrm{x}}\right), 8.26\left(\mathrm{~d}, J=5.6 \mathrm{~Hz}, 8 \mathrm{H}, \mathrm{H}_{\mathrm{b}}\right), 8.16\left(\mathrm{~d}, J=8.3 \mathrm{~Hz}, 4 \mathrm{H}, \mathrm{H}_{\mathrm{y}}\right)$, 8.09 (d, $\left.J=8.4 \mathrm{~Hz}, 4 \mathrm{H}, \mathrm{H}_{\mathrm{z}}\right), 6.79$ (s, 4H, $\left.\mathrm{H}_{\mathrm{c}^{\prime}}\right), 2.35-2.24$ (m, 48H, $\left.\mathrm{H}_{-\mathrm{CH}_{2}}\right), 1.49$ (m, 72H, $\left.\mathrm{H}_{-\mathrm{CH} 3}\right)$ ppm. ${ }^{31} \mathrm{P}$ NMR $\left(203 \mathrm{MHz}\right.$, acetone- $\left.d_{6}\right) \delta=6.13(\mathrm{~d}, J=21.0 \mathrm{~Hz}), 1.09(\mathrm{~d}, J=20.8 \mathrm{~Hz}) \mathrm{ppm}$. ESI-MS $(\mathrm{m} / \mathrm{z})$ Calculated for $\mathrm{C}_{114} \mathrm{H}_{156} \mathrm{~F}_{6} \mathrm{~N}_{8} \mathrm{O}_{14} \mathrm{P}_{8} \mathrm{Pt}_{4} \mathrm{~S}_{2} \mathrm{Zn}[\mathrm{M}-2 \mathrm{TfO}]^{2+}$ : 1567.19. Found: 1567.05 .

BT1'. cis-Pt(PEt $)_{2}(\mathrm{OTf})_{2}(4.47 \mathrm{mg}, 0.0064 \mathrm{mmol}), \mathrm{HDC}(0.72 \mathrm{mg}, 0.0032 \mathrm{mmol})$ and TPP (1 mg, 0.00162 $\mathrm{mmol})$ in a 1:1:1 DCM/MeNO$/ \mathrm{MeCN}_{2}$ solvent mixture $(1 \mathrm{~mL}) .{ }^{1} \mathrm{H}$ NMR $\left(500 \mathrm{MHz}\right.$, acetone- $\left.d_{6}\right) \delta=9.39$ (br s, 4H, $\left.\mathrm{H}_{\mathrm{c}^{\prime \prime}}\right), 9.37-9.31\left(\mathrm{~m}, 8 \mathrm{H}, \mathrm{H}_{\mathrm{a}}\right), 8.59$ (d, $\left.J=6.4 \mathrm{~Hz}, 8 \mathrm{H}, \mathrm{H}_{\mathrm{b}}\right), 8.37\left(\mathrm{~s}, 4 \mathrm{H}, \mathrm{H}_{\mathrm{c}^{\prime}}\right), 2.40-2.16(\mathrm{~m}, 56 \mathrm{H}$, $\mathrm{H}_{\mathrm{s}}$ and $\left.\mathrm{H}_{-\mathrm{CH} 2-}\right), 1.66\left(\right.$ br s, 8H, $\left.\mathrm{H}_{\mathrm{t}}\right), 1.55-1.36\left(\mathrm{~m}, 72 \mathrm{H}, \mathrm{H}_{-\mathrm{CH}}\right),-2.97\left(\mathrm{~s}, 2 \mathrm{H}, \mathrm{H}_{\mathrm{d}}\right) .{ }^{31} \mathrm{P} \mathrm{NMR}(203 \mathrm{MHz}$, acetone- $\left.d_{6}\right) \delta=6.27(\mathrm{~d}, J=21.1 \mathrm{~Hz}), 0.26(\mathrm{~d}, J=21.2 \mathrm{~Hz}) \mathrm{ppm}$.

BT2' ${ }^{\prime}$ cis-Pt(PEt $)_{2}(\mathrm{OTf})_{2}(14.1 \mathrm{mg}, 0.019 \mathrm{mmol}), \mathrm{BPDC}$ (3.08 mg, $\left.0.0096 \mathrm{mmol}\right)$ and TPP (3.0 mg, 0.0048 $\mathrm{mmol})$ in a $1: 1: 1 \mathrm{DCM} / \mathrm{MeNO}_{2} / \mathrm{MeCN}$ solvent mixture $(5 \mathrm{~mL}) .{ }^{1} \mathrm{H}$ NMR $\left(500 \mathrm{MHz}\right.$, acetone- $\left.d_{6}\right) \delta=9.34$ (brs, $\left.8 \mathrm{H}, \mathrm{H}_{\mathrm{a}}\right), 8.58\left(\mathrm{~s}, 4 \mathrm{H}, \mathrm{H}_{\mathrm{c}^{\prime \prime}}\right), 8.38\left(\mathrm{~d}, J=5.6 \mathrm{~Hz}, 8 \mathrm{H}, \mathrm{H}_{\mathrm{b}}\right), 8.10\left(\mathrm{~d}, J=8.1 \mathrm{~Hz}, 8 \mathrm{H}, \mathrm{H}_{\mathrm{u}}\right), 7.98(\mathrm{~d}, J=8.2$ $\left.\mathrm{Hz}, 8 \mathrm{H}, \mathrm{H}_{\mathrm{v}}\right), 7.74\left(\mathrm{~s}, 4 \mathrm{H}, \mathrm{H}_{\mathrm{c}^{\prime}}\right), 2.44-2.18\left(\mathrm{~m}, 48 \mathrm{H}, \mathrm{H}_{-\mathrm{CH} 2}\right), 1.58-1.42\left(\mathrm{~m}, 72 \mathrm{H}, \mathrm{H}_{-\mathrm{CH} 3}\right),-3.31\left(\mathrm{~s}, 2 \mathrm{H}, \mathrm{H}_{\mathrm{d}}\right)$. 
${ }^{31} \mathrm{P}$ NMR $\left(202 \mathrm{MHz}\right.$, acetone- $\left.d_{6}\right) \delta=6.37(\mathrm{~d}, J=21.0 \mathrm{~Hz}), 1.28(\mathrm{~d}, J=21.2 \mathrm{~Hz}) \mathrm{ppm}$. ESI-MS $(m / z)$ Calculated for $\mathrm{C}_{118} \mathrm{H}_{162} \mathrm{~F}_{6} \mathrm{~N}_{8} \mathrm{O}_{14} \mathrm{P}_{8} \mathrm{Pt}_{4} \mathrm{~S}_{2}[\mathrm{M}-2 \mathrm{TfO}]^{2+}$ : 1561.55. Found: 1561.60 .

BT3 $^{\prime}$. cis-Pt( $\left(\mathrm{PEt}_{3}\right)_{2}(\mathrm{OTf})_{2}(14.1 \mathrm{mg}, 0.019 \mathrm{mmol})$, BDC (2.34 mg, $\left.0.0096 \mathrm{mmol}\right)$ and TPP (3.0 mg, 0.0048 $\mathrm{mmol})$ in a 1:1:1 DCM/MeNO$/ \mathrm{MeCN}$ solvent mixture $(5 \mathrm{~mL}) .{ }^{1} \mathrm{H}$ NMR $\left(500 \mathrm{MHz}\right.$, acetone- $\left.d_{6}\right) \delta=9.40$ $\left(\mathrm{d}, J=6.1 \mathrm{~Hz}, 8 \mathrm{H}, \mathrm{H}_{\mathrm{a}}\right), 9.31\left(\mathrm{~s}, 4 \mathrm{H}, \mathrm{H}_{\mathrm{c}^{\prime \prime}}\right), 8.43\left(\mathrm{~d}, J=5.5 \mathrm{~Hz}, 8 \mathrm{H}, \mathrm{H}_{\mathrm{b}}\right), 8.08\left(\mathrm{~s}, 8 \mathrm{H}, \mathrm{H}_{\mathrm{w}}\right), 7.08\left(\mathrm{~s}, 4 \mathrm{H}, \mathrm{H}_{\mathrm{c}^{\prime}}\right)$, $2.40-2.18\left(\mathrm{~m}, 48 \mathrm{H}, \mathrm{H}_{-\mathrm{CH} 2}\right), 1.61-1.38\left(\mathrm{~m}, 72 \mathrm{H}, \mathrm{H}_{-\mathrm{CH} 3}\right),-3.27\left(\mathrm{~s}, 2 \mathrm{H}, \mathrm{H}_{\mathrm{d}}\right) \mathrm{ppm} .{ }^{31} \mathrm{P}$ NMR (122 MHz, acetone- $\left.d_{6}\right) \delta=6.73(\mathrm{~d}, J=20.6 \mathrm{~Hz}), 1.73(\mathrm{~d}, J=21.2 \mathrm{~Hz}) \mathrm{ppm}$. ESI-MS $(\mathrm{m} / \mathrm{z})$ Calculated for $\mathrm{C}_{106} \mathrm{H}_{154} \mathrm{~F}_{6} \mathrm{~N}_{8} \mathrm{O}_{14} \mathrm{P}_{8} \mathrm{Pt}_{4} \mathrm{~S}_{2}[\mathrm{M}-2 \mathrm{TfO}]^{2+}$ : 1485.45. Found: 1485.52.

BT4' . cis-Pt(PEt $)_{2}(\mathrm{OTf})_{2}(14.1 \mathrm{mg}, 0.019 \mathrm{mmol}), \mathrm{BDC}$ (2.83 mg, $\left.0.0096 \mathrm{mmol}\right)$ and TPP (3.0 mg, 0.0048 $\mathrm{mmol})$ in a 1:1:1 $\mathrm{DCM} / \mathrm{MeNO}_{2} / \mathrm{MeCN}$ solvent mixture $(5 \mathrm{~mL}) .{ }^{1} \mathrm{H} \mathrm{NMR}\left(500 \mathrm{MHz}, \mathrm{CD}_{3} \mathrm{NO}_{2}\right) \delta=9.33$ (br $\left.\mathrm{s}, 8 \mathrm{H}, \mathrm{H}_{\mathrm{a}}\right), 8.88\left(\mathrm{~s}, 4 \mathrm{H}, \mathrm{H}_{\mathrm{c}^{\prime \prime}}\right), 8.73\left(\mathrm{~d}, J=1.6 \mathrm{~Hz}, 4 \mathrm{H}, \mathrm{H}_{\mathrm{x}}\right), 8.28\left(\mathrm{~d}, J=5.5 \mathrm{~Hz}, 8 \mathrm{H}, \mathrm{H}_{\mathrm{b}}\right), 8.18(\mathrm{~d}, J=8.3 \mathrm{~Hz}$, $\left.4 \mathrm{H}, \mathrm{H}_{\mathrm{y}}\right), 8.11\left(\mathrm{~d}, J=8.4 \mathrm{~Hz}, 4 \mathrm{H}, \mathrm{H}_{\mathrm{z}}\right), 6.80\left(\mathrm{~s}, 4 \mathrm{H}, \mathrm{H}_{\mathrm{c}^{\prime}}\right), 2.29$ (m, 48H, $\left.\mathrm{H}_{-\mathrm{CH} 2-}\right), 1.53\left(\mathrm{~m}, 72 \mathrm{H}_{1} \mathrm{H}_{-\mathrm{CH} 3}\right),-3.42$ $\left(\mathrm{s}, 2 \mathrm{H}, \mathrm{H}_{\mathrm{d}}\right)$ ppm. ${ }^{31} \mathrm{P} \mathrm{NMR}\left(203 \mathrm{MHz}, \mathrm{CD}_{3} \mathrm{NO}_{2}\right) \delta=6.52(\mathrm{~d}, J=21.2 \mathrm{~Hz}), 1.07(\mathrm{~d}, J=21.1 \mathrm{~Hz}) \mathrm{ppm}$. ESIMS $(m / z)$ Calculated for $\mathrm{C}_{114} \mathrm{H}_{158} \mathrm{~F}_{6} \mathrm{~N}_{8} \mathrm{O}_{14} \mathrm{P}_{8} \mathrm{Pt}_{4} \mathrm{~S}_{2}[\mathrm{M}-2 \mathrm{TfO}]^{2+}: 1535.51$. Found: 1535.60 . 


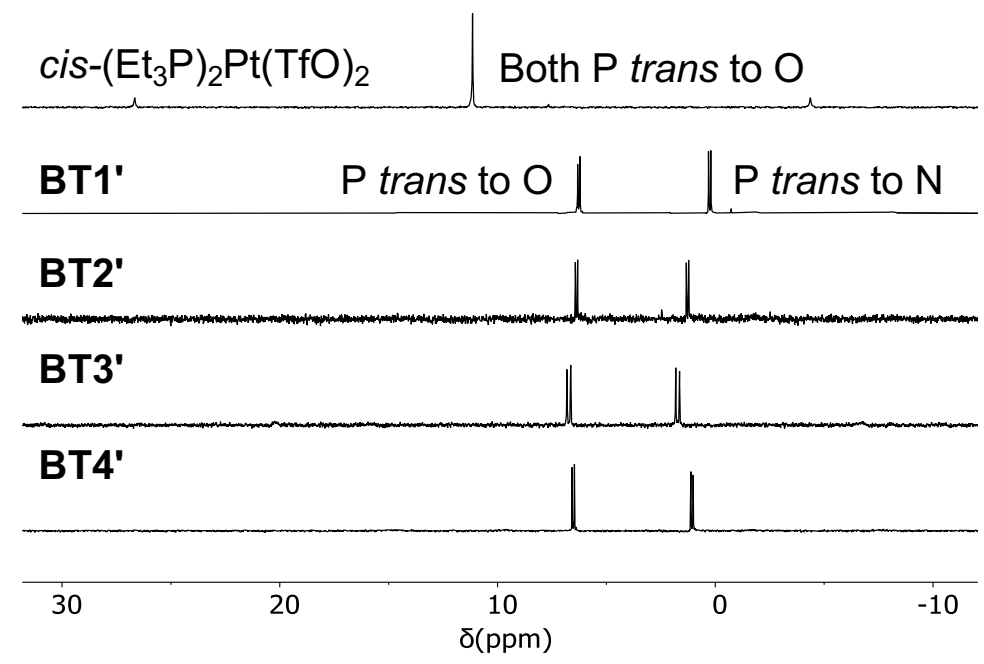

Figure S1. The ${ }^{31} \mathrm{P}$ NMR spectra (122 MHz, acetone- $\left.d_{6}\right)$ of $c i s-\left(\mathrm{Et}_{3} \mathrm{P}\right)_{2} \mathrm{Pt}(\mathrm{TfO})$, BT1', BT2', BT3', and BT4' complexes reveal simultaneous coordination of carboxylate and pyridyl groups with heteroligated $\mathrm{Pt}(\mathrm{N}, \mathrm{O})$ corners in the bow tie complexes.

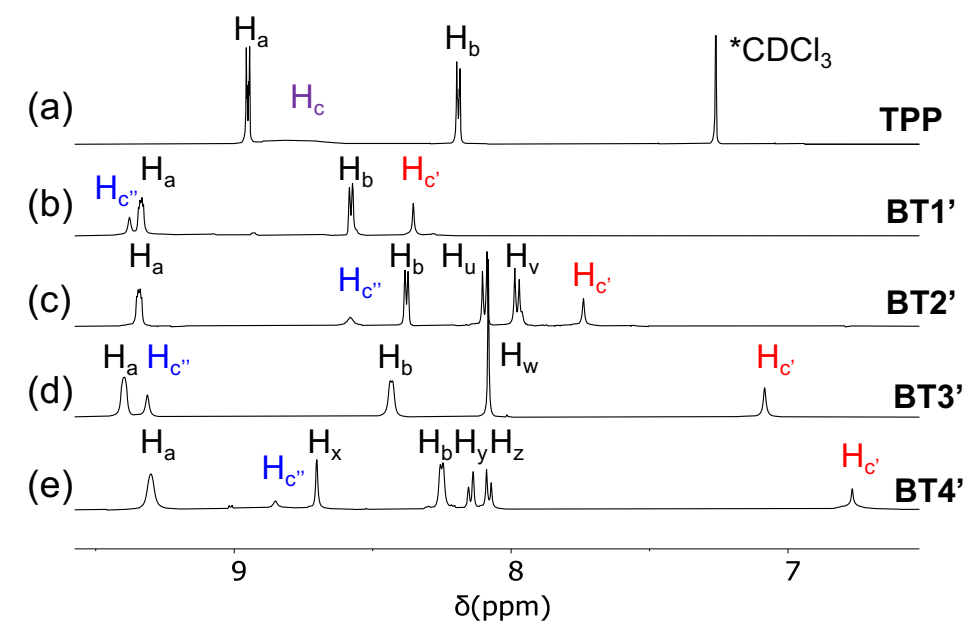

Figure S2. The partial ${ }^{1} \mathrm{H}$ NMR spectra (500 MHz) of (a) free $\mathrm{H}_{2} \mathrm{TPP}$ ligand (in $\mathrm{CDCl}_{3}$ ), (b) BT1', (c) BT2', (d) BT3', and (e) BT4' complexes (in acetone- $d_{6}$ ) show that the enclosed $\mathrm{H}_{\mathrm{c}^{\prime}}$ pyrrole protons (highlighted in red) of bow tie complexes are shifted up-field commensurately with the shielding effect of the adjacent XDC linkers, whereas the chemical shifts of exposed $\mathrm{H}_{\mathrm{c}^{\prime \prime}}$ pyrrole protons (highlighted in blue) vary depending on the length of XDC linkers that dictate their distances from the adjacent pyridyl rings. 

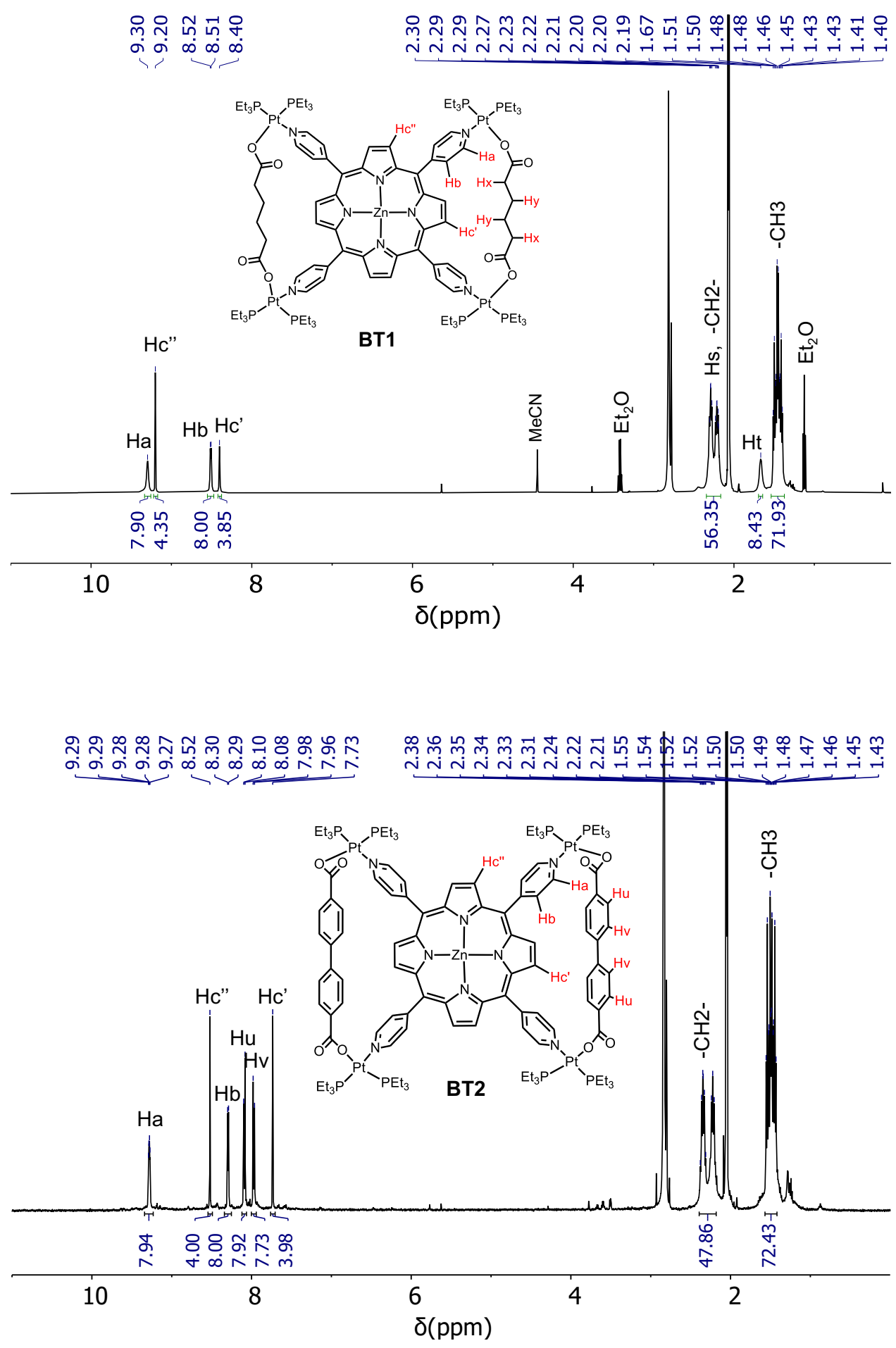


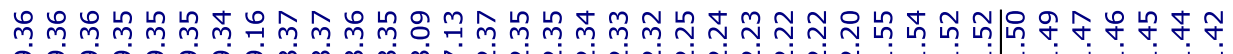
कं
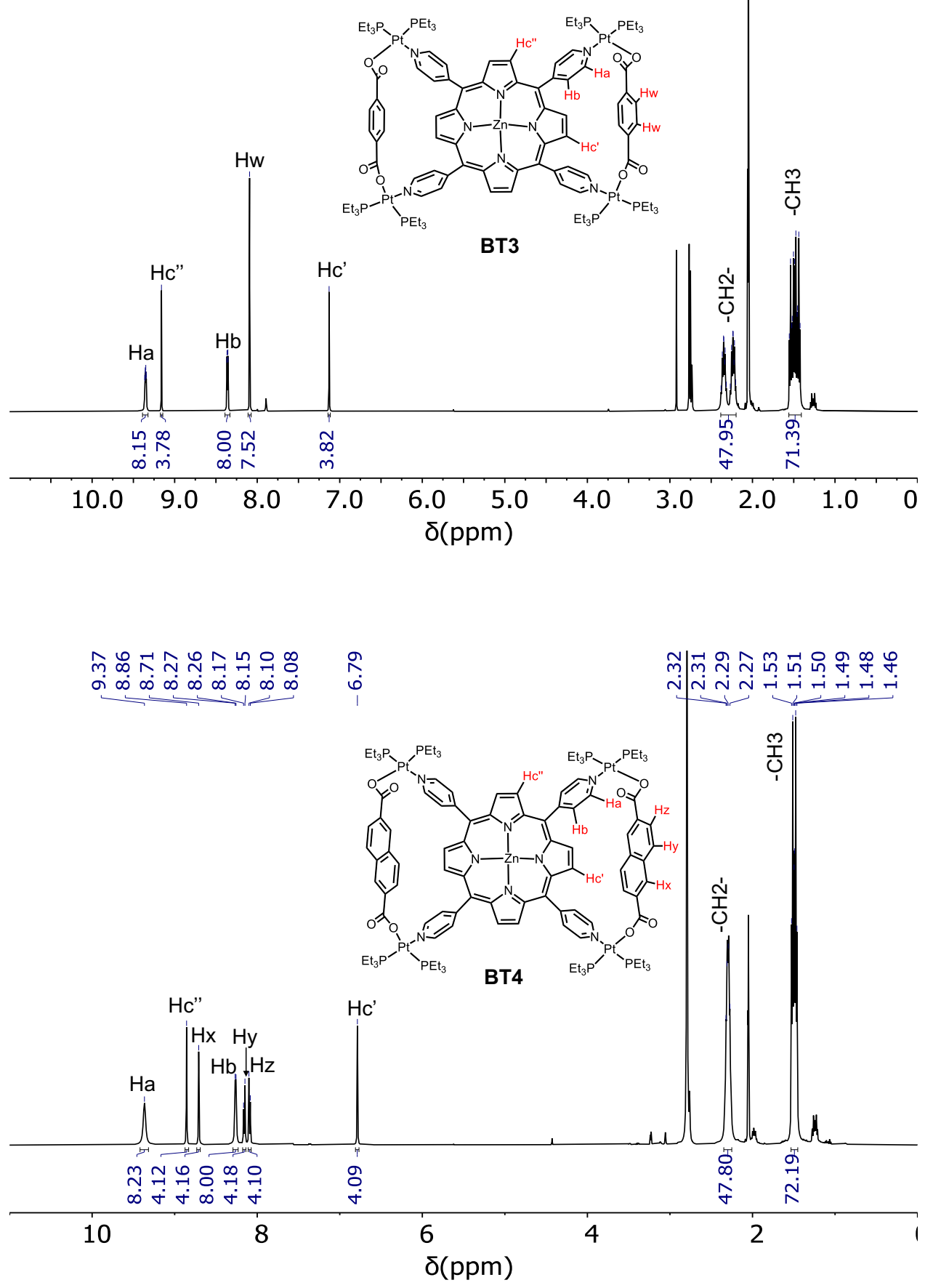
mి

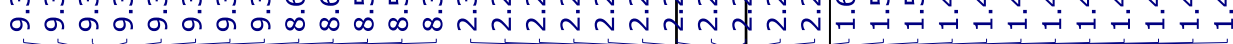

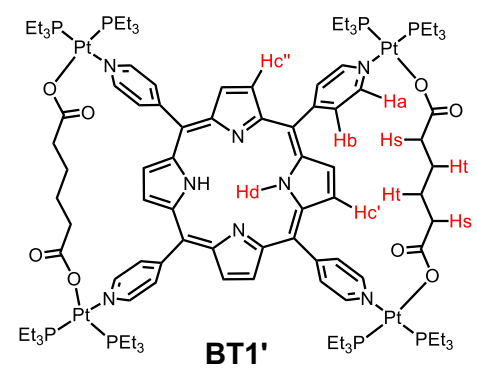
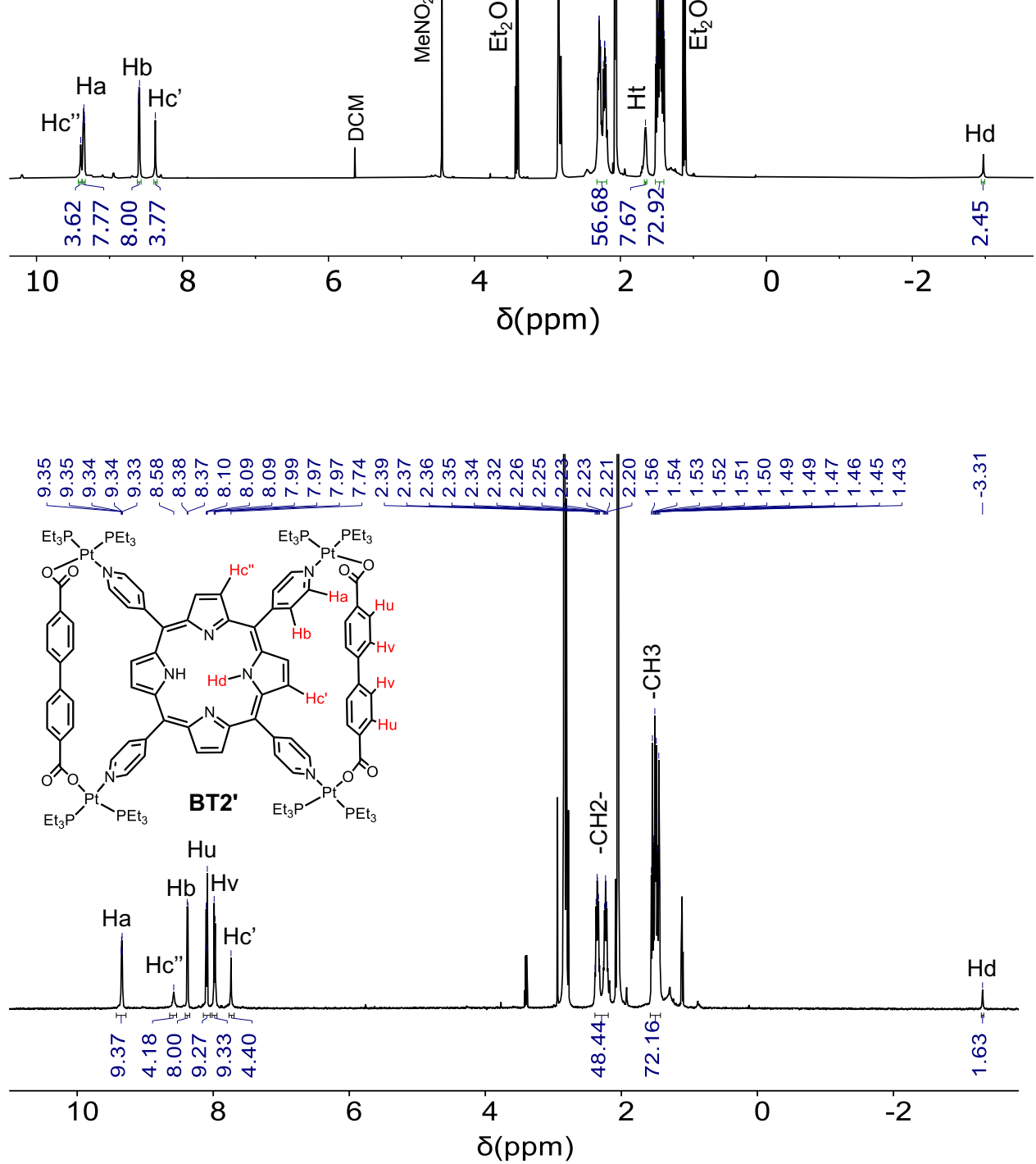

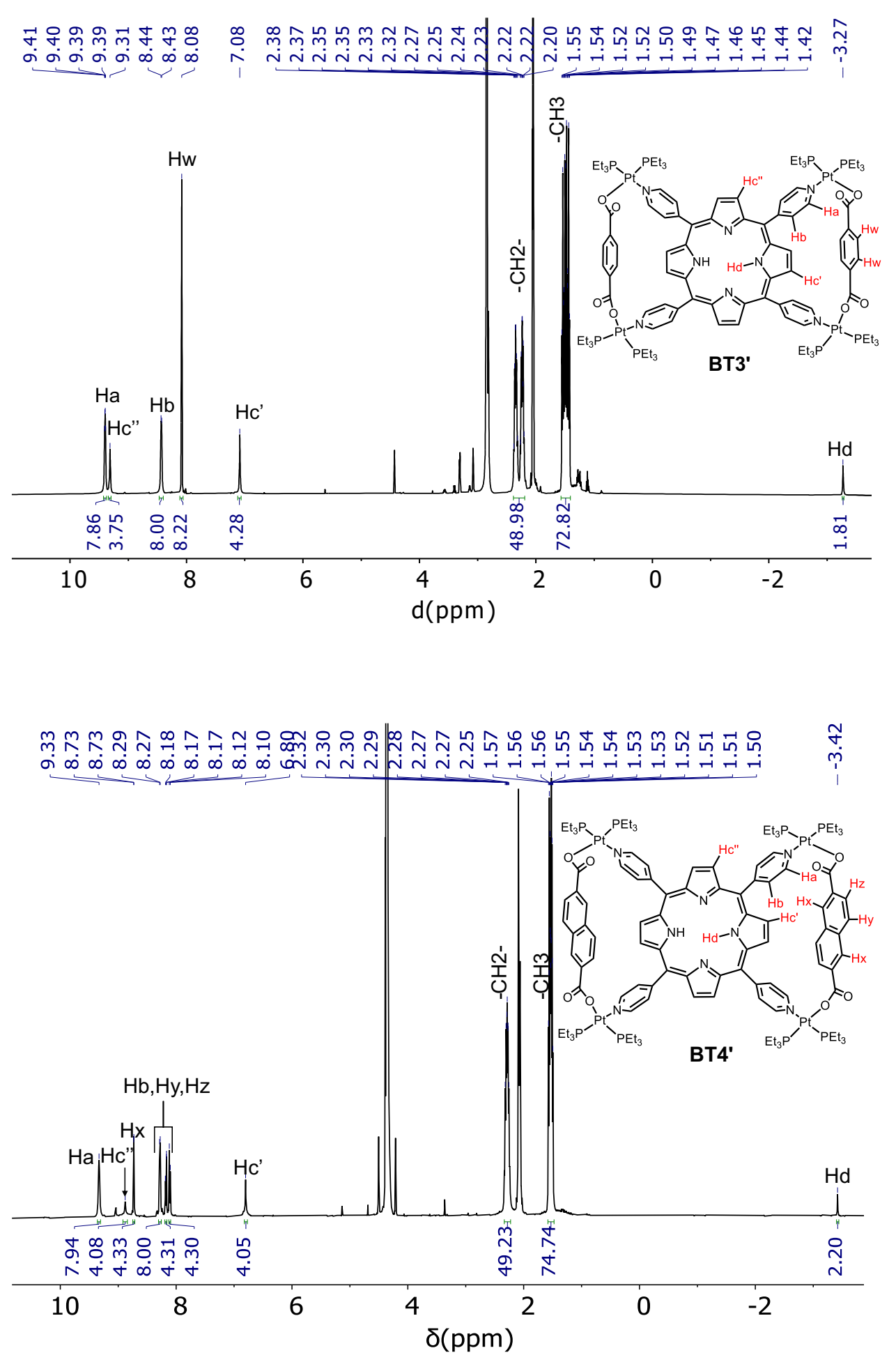

Figure S3. The ${ }^{1} \mathrm{H}$ NMR spectra $\left(500 \mathrm{MHz}\right.$, acetone- $\left.d_{6}\right)$ of BT1-BT4 and BT1'-BT4' complexes. 


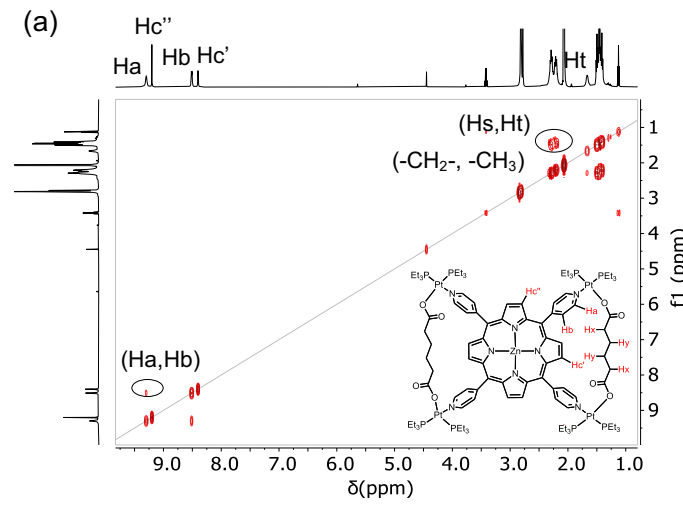

(b)
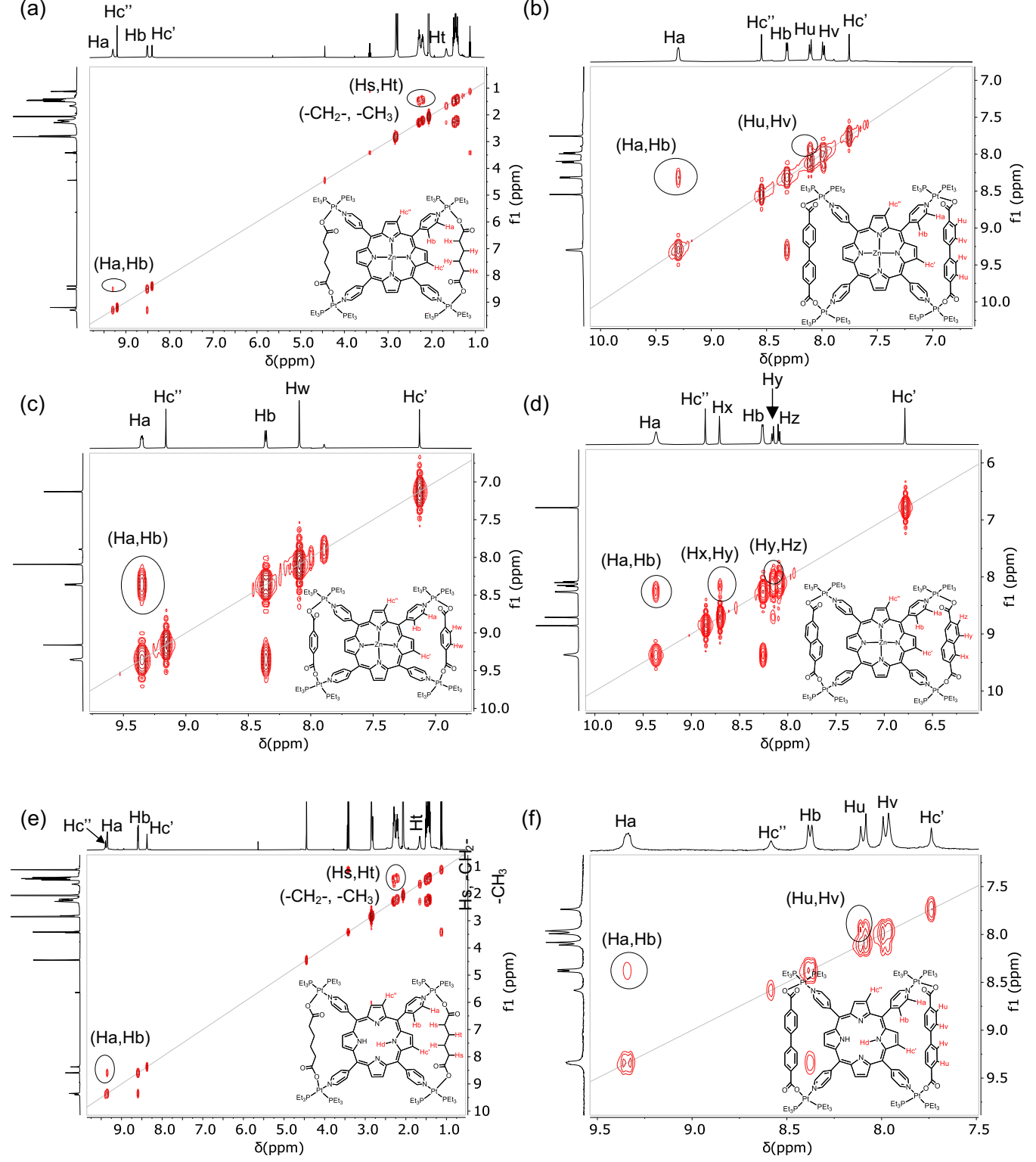

(g)
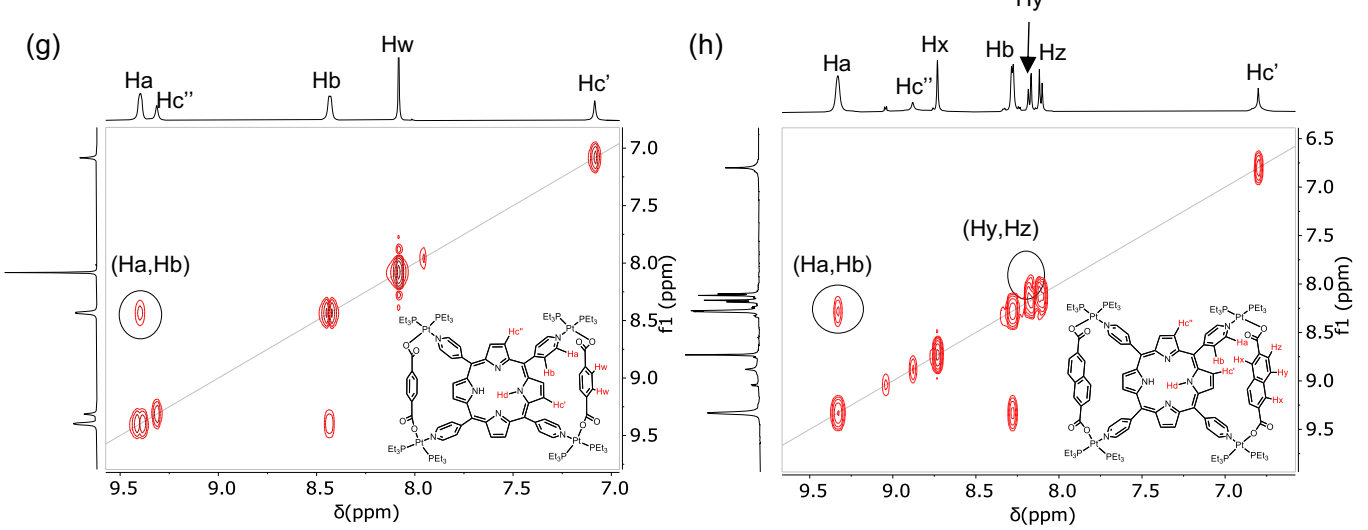

Figure S4. The ${ }^{1} \mathrm{H}-{ }^{1} \mathrm{H}$ COSY NMR spectra $\left(500 \mathrm{MHz}\right.$, acetone- $\left.d_{6}\right)$ of (a-d) BT1-BT4 and (e-h) BT1'BT4' complexes. 

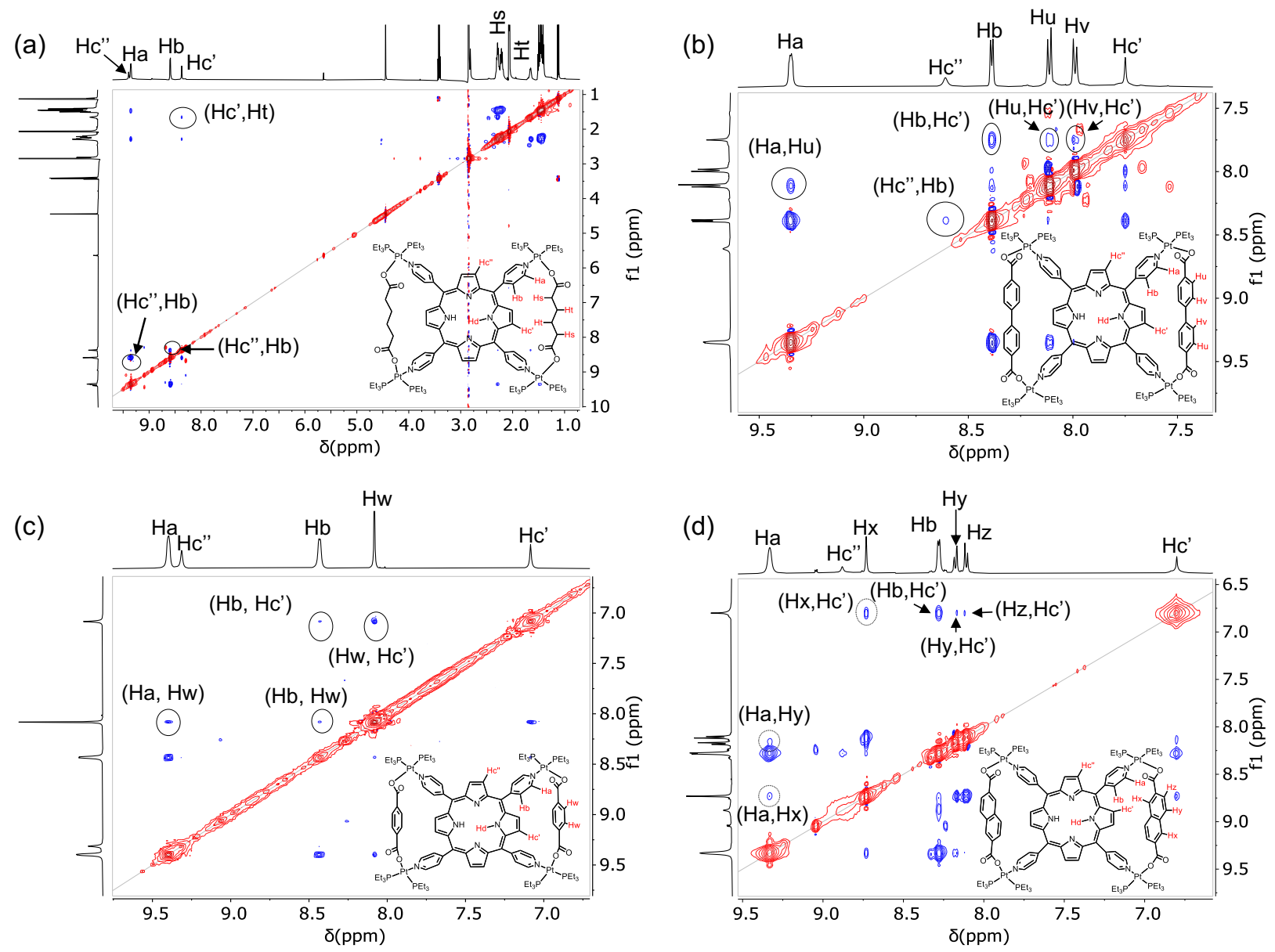

Figure S5. The ${ }^{1} \mathrm{H}-{ }^{1} \mathrm{H}$ ROESY NMR spectra (500 MHz, acetone- $d_{6}$ ) of (a) BT1', (b) BT2', (c) BT3', and (d) BT4' complexes. 

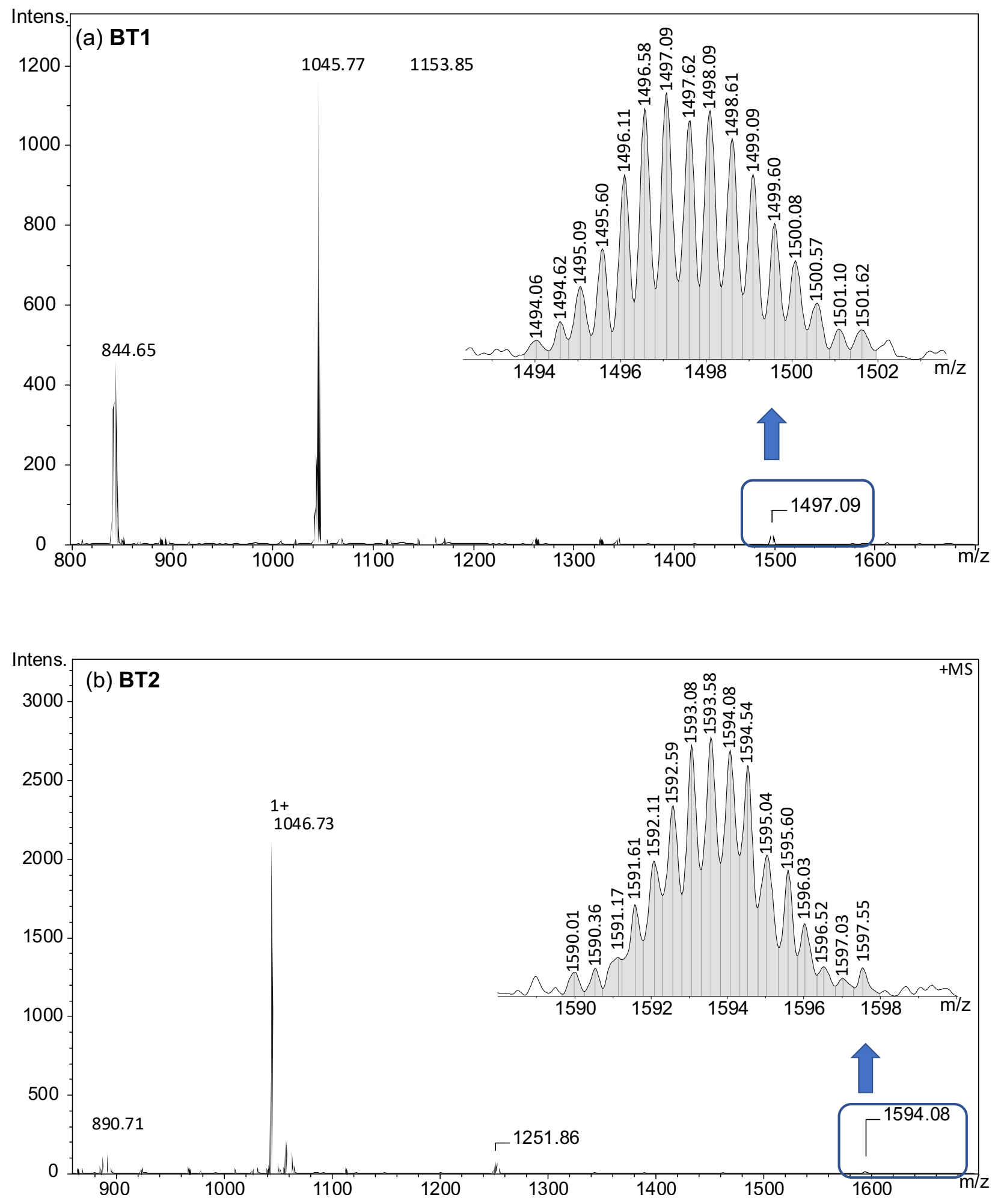

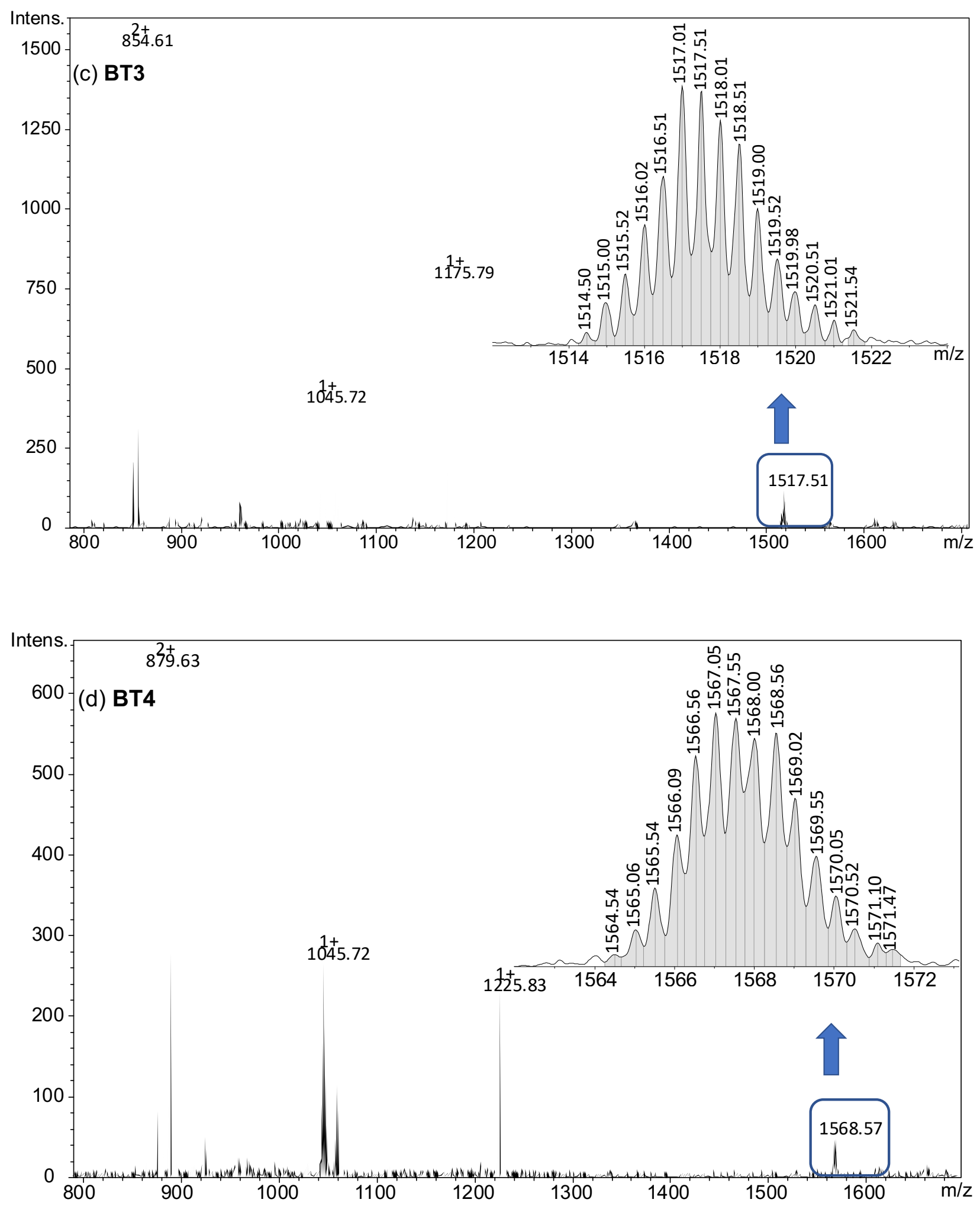

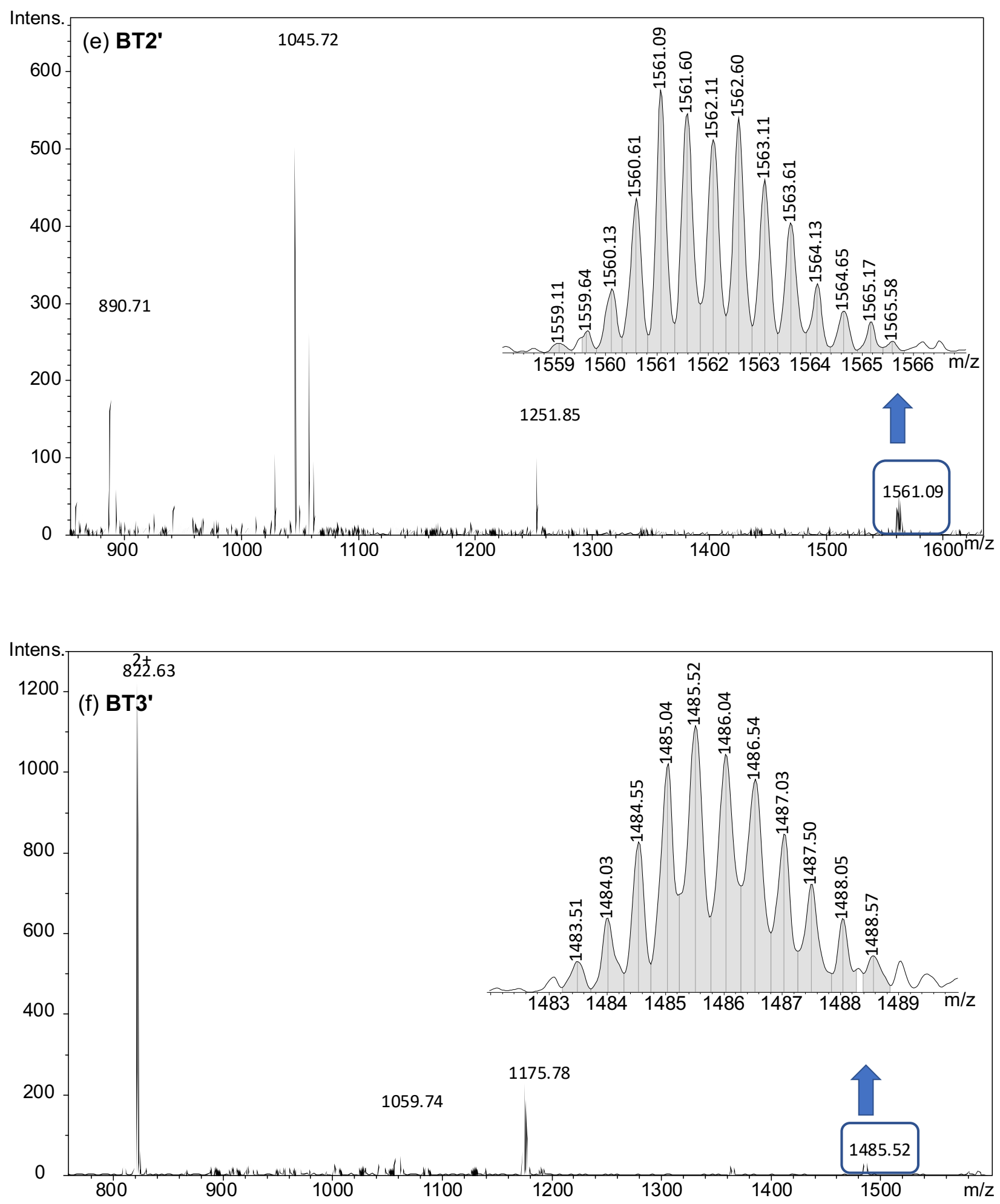


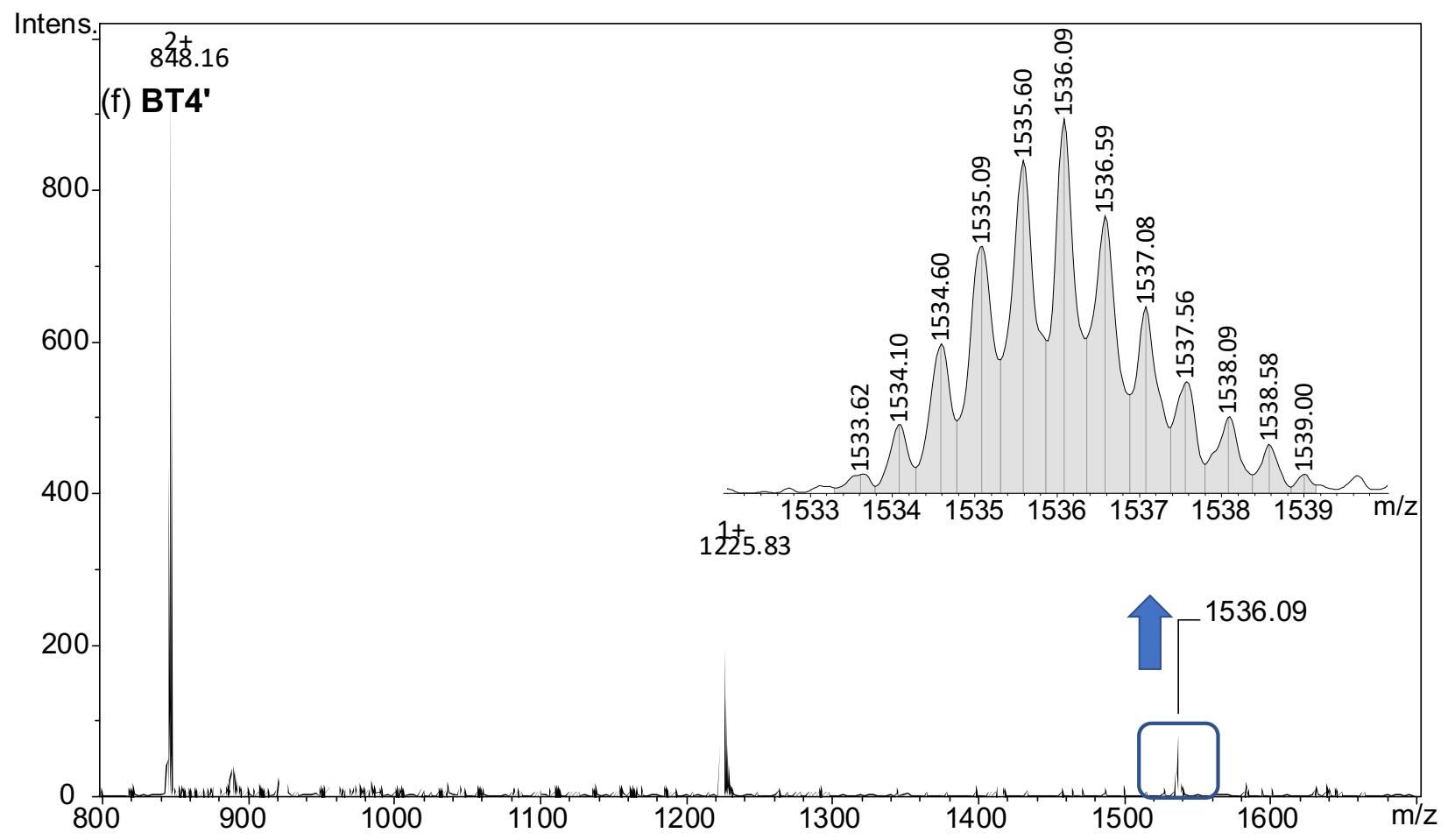

Figure S6. The ESI-MS data of (a) BT1, (b) BT2, (c) BT3, (d) BT4, (e) BT2', (f) BT3', and (g) BT4' complexes. The insets show the isotopic distributions of respective $[\mathrm{M}-2 \mathrm{TfO}]^{2+}$ species. 


\section{Crystallographic Data.}

Table 1. Crystallographic Data for BT2, BT3 and BT4.

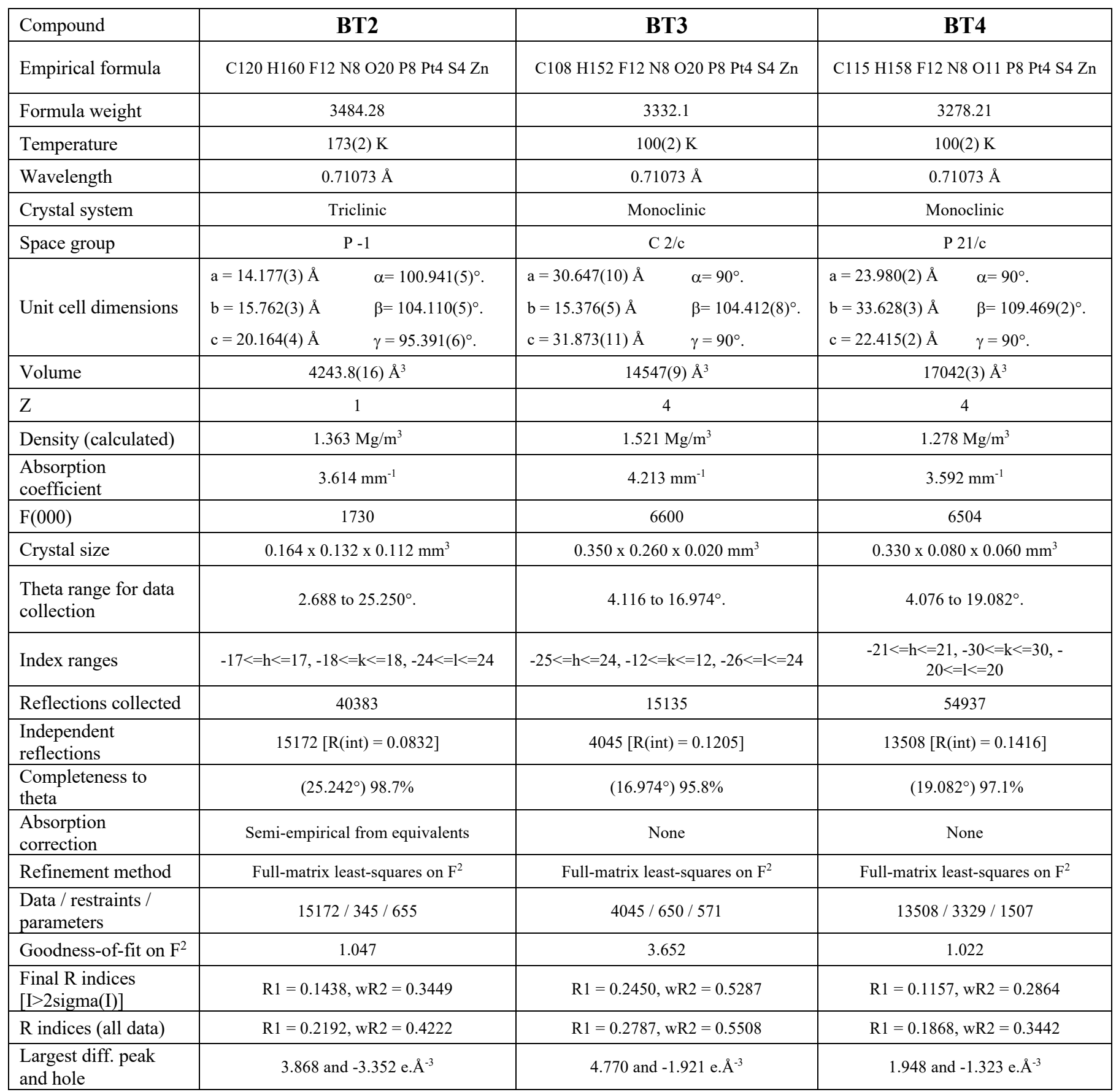


Table 2. Crystallographic Data for BT2' and BT3'.

\begin{tabular}{|c|c|c|}
\hline Compound & BT2' & BT3' \\
\hline Empirical formula & C123 H168 F12 N8 O21 P8 Pt4 S4 & C108 H150 F12 N8 O20 P8 Pt4 S4 \\
\hline Formula weight & 3479 & 3264.71 \\
\hline Temperature & $100(2) \mathrm{K}$ & $100(2) \mathrm{K}$ \\
\hline Wavelength & $0.71073 \AA$ & $0.71073 \AA$ \\
\hline Crystal system & Monoclinic & Tetragonal \\
\hline Space group & $\mathrm{C} 2 / \mathrm{c}$ & I $41 / \mathrm{a}$ \\
\hline Unit cell dimensions & $\begin{array}{ll}\mathrm{a}=42.500(3) \AA & \alpha=90^{\circ} . \\
\mathrm{b}=17.5276(14) \AA & \beta=107.338(2)^{\circ} . \\
\mathrm{c}=22.8229(18) \AA & \gamma=90^{\circ} .\end{array}$ & $\begin{array}{ll}\mathrm{a}=34.285(6) \AA & \alpha=90^{\circ} . \\
\mathrm{b}=34.285(6) \AA & \beta=90^{\circ} . \\
\mathrm{c}=23.949(5) \AA & \gamma=90^{\circ} .\end{array}$ \\
\hline Volume & $16229(2) \AA^{3}$ & $28151(11) \AA^{3}$ \\
\hline Z & 4 & 8 \\
\hline Density (calculated) & $1.424 \mathrm{Mg} / \mathrm{m}^{3}$ & $1.541 \mathrm{Mg} / \mathrm{m}^{3}$ \\
\hline Absorption coefficient & $3.638 \mathrm{~mm}^{-1}$ & $4.188 \mathrm{~mm}^{-1}$ \\
\hline $\mathrm{F}(000)$ & 6936 & 12944 \\
\hline Crystal size & $0.160 \times 0.120 \times 0.030 \mathrm{~mm}^{3}$ & $0.18 \times 0.12 \times 0.04 \mathrm{~mm}^{3}$ \\
\hline $\begin{array}{l}\text { Theta range for data } \\
\text { collection }\end{array}$ & 2.309 to $25.250^{\circ}$. & 2.304 to $17.312^{\circ}$. \\
\hline Index ranges & $-50<=\mathrm{h}<=50,-20<=\mathrm{k}<=21,-27<=1<=27$ & $-28<=\mathrm{h}<=28,-28<=\mathrm{k}<=28,-20<=1<=19$ \\
\hline Reflections collected & 48255 & 33537 \\
\hline Independent reflections & $14608[\mathrm{R}$ (int) $=0.0829]$ & $4010[\mathrm{R}(\mathrm{int})=0.1656]$ \\
\hline Completeness to theta & $\left(25.242^{\circ}\right) 99.4 \%$ & $\left(17.312^{\circ}\right) 92.7 \%$ \\
\hline Absorption correction & Semi-empirical from equivalents & Semi-empirical from equivalents \\
\hline Refinement method & Full-matrix least-squares on $\mathrm{F}^{2}$ & Full-matrix least-squares on $\mathrm{F}^{2}$ \\
\hline $\begin{array}{l}\text { Data / restraints / } \\
\text { parameters }\end{array}$ & $14608 / 96 / 824$ & $4010 / 1227 / 696$ \\
\hline Goodness-of-fit on F2 & 1.065 & 1.361 \\
\hline $\begin{array}{l}\text { Final R indices } \\
{[\mathrm{I}>2 \operatorname{sigma}(\mathrm{I})]}\end{array}$ & $\mathrm{R} 1=0.0850, \mathrm{wR} 2=0.1982$ & $\mathrm{R} 1=0.1104, \mathrm{wR} 2=0.2814$ \\
\hline $\mathrm{R}$ indices (all data) & $\mathrm{R} 1=0.1100, \mathrm{wR} 2=0.2126$ & $\mathrm{R} 1=0.1786, \mathrm{wR} 2=0.3603$ \\
\hline Largest diff. peak and hole & 3.151 and -3.752 e. $\AA \AA-3$ & 2.357 and -0.946 e. $\AA-3$ \\
\hline
\end{tabular}


Table 3. Atomic coordinates ( $\left.\times 10^{4}\right)$ and equivalent isotropic displacement parameters $\left(\AA^{2} \times 10^{3}\right)$ for BT2. U(eq) is defined as one third of the trace of the orthogonalized $U^{i j}$ tensor.

\begin{tabular}{lrrrr}
\hline & \multicolumn{1}{c}{$\mathrm{y}$} & $\mathrm{U}(\mathrm{eq})$ \\
\hline $\mathrm{Pt}(1)$ & $7791(1)$ & $5049(1)$ & $7517(1)$ & $63(1)$ \\
$\mathrm{Pt}(2)$ & $3947(1)$ & $-1436(1)$ & $1101(1)$ & $70(1)$ \\
$\mathrm{Zn}(1)$ & 10000 & 0 & 5000 & $87(2)$ \\
$\mathrm{P}(1)$ & $6998(7)$ & $6182(5)$ & $7805(5)$ & $93(3)$ \\
$\mathrm{P}(2)$ & $9278(5)$ & $5624(5)$ & $8225(4)$ & $62(2)$ \\
$\mathrm{P}(3)$ & $4493(5)$ & $-2410(6)$ & $329(5)$ & $82(2)$ \\
$\mathrm{P}(4)$ & $2484(6)$ & $-1474(6)$ & $334(5)$ & $86(3)$ \\
$\mathrm{O}(1)$ & $6183(15)$ & $3477(13)$ & $7453(10)$ & $81(4)$ \\
$\mathrm{O}(2)$ & $6454(14)$ & $4549(13)$ & $6813(9)$ & $70(4)$ \\
$\mathrm{O}(3)$ & $3596(14)$ & $-445(11)$ & $1769(12)$ & $79(4)$ \\
$\mathrm{O}(4)$ & $2973(15)$ & $-1373(12)$ & $2334(12)$ & $86(5)$ \\
$\mathrm{N}(1)$ & $10534(15)$ & $864(13)$ & $5922(11)$ & $63(5)$ \\
$\mathrm{N}(2)$ & $8687(16)$ & $512(15)$ & $4855(12)$ & $74(6)$ \\
$\mathrm{N}(3)$ & $8275(13)$ & $3898(11)$ & $7029(12)$ & $54(4)$ \\
$\mathrm{N}(4)$ & $5266(14)$ & $-1203(17)$ & $1928(12)$ & $69(5)$ \\
$\mathrm{C}(1)$ & $11409(18)$ & $978(17)$ & $6387(13)$ & $61(6)$ \\
$\mathrm{C}(2)$ & $11493(19)$ & $1613(19)$ & $6990(14)$ & $72(6)$ \\
$\mathrm{C}(3)$ & $10665(18)$ & $1948(19)$ & $6923(14)$ & $72(6)$ \\
$\mathrm{C}(4)$ & $10039(18)$ & $1480(19)$ & $6230(13)$ & $67(6)$ \\
$\mathrm{C}(5)$ & $9068(19)$ & $1630(17)$ & $5941(14)$ & $63(7)$ \\
$\mathrm{C}(6)$ & $8453(18)$ & $1163(18)$ & $5286(14)$ & $66(6)$ \\
$\mathrm{C}(7)$ & $7461(18)$ & $1337(18)$ & $4973(14)$ & $66(6)$ \\
$\mathrm{C}(8)$ & $7080(20)$ & $686(18)$ & $4337(14)$ & $68(6)$ \\
$\mathrm{C}(9)$ & $7884(18)$ & $219(18)$ & $4269(13)$ & $63(6)$ \\
$\mathrm{C}(10)$ & $7800(20)$ & $-453(19)$ & $3724(13)$ & $69(8)$ \\
$\mathrm{C}(11)$ & $8725(16)$ & $2421(17)$ & $6313(13)$ & $58(7)$ \\
$\mathrm{C}(12)$ & $8780(30)$ & $3140(20)$ & $6086(17)$ & $102(13)$ \\
$\mathrm{C}(13)$ & $8484(16)$ & $3931(14)$ & $6503(15)$ & $55(4)$ \\
$\mathrm{C}(14)$ & $8205(16)$ & $3181(14)$ & $7297(15)$ & $55(4)$ \\
$\mathrm{C}(15)$ & $8453(17)$ & $2389(15)$ & $6934(11)$ & $50(6)$ \\
$\mathrm{C}(16)$ & $6896(16)$ & $-677(17)$ & $3078(12)$ & $52(6)$ \\
$\mathrm{C}(17)$ & $6700(20)$ & $-200(20)$ & $2591(16)$ & $92(11)$ \\
$\mathrm{C}(18)$ & $5831(17)$ & $-440(20)$ & $2029(15)$ & $70(5)$ \\
$\mathrm{C}(19)$ & $5407(17)$ & $-1660(20)$ & $2401(15)$ & $69(5)$ \\
$\mathrm{C}(20)$ & $6210(20)$ & $-1450(20)$ & $3002(16)$ & $84(9)$ \\
$\mathrm{C}(21)$ & $6110(20)$ & $3760(20)$ & $6923(15)$ & $71(4)$ \\
$\mathrm{C}(22)$ & $5520(30)$ & $3115(16)$ & $6249(16)$ & $92(4)$
\end{tabular}




\begin{tabular}{|c|c|c|c|c|}
\hline$C(23)$ & $5470(30)$ & $2225(15)$ & $6229(16)$ & $91(4)$ \\
\hline$C(24)$ & $5090(30)$ & $1650(20)$ & $5586(12)$ & $91(4)$ \\
\hline$C(25)$ & $4740(30)$ & $1890(16)$ & $4957(15)$ & $91(4)$ \\
\hline$C(26)$ & $4770(30)$ & $2781(15)$ & 4991(16) & $93(4)$ \\
\hline$C(27)$ & $5180(30)$ & $3380(20)$ & $5627(12)$ & $93(4)$ \\
\hline$C(28)$ & $4289(19)$ & $1324(17)$ & $4297(15)$ & $89(4)$ \\
\hline$C(29)$ & $3712(17)$ & $507(15)$ & $4243(15)$ & $89(4)$ \\
\hline$C(30)$ & $3430(20)$ & $-138(19)$ & $3608(12)$ & $89(4)$ \\
\hline$C(31)$ & $3670(20)$ & $10(18)$ & $2989(15)$ & $89(4)$ \\
\hline$C(32)$ & $4300(20)$ & 797(16) & $3058(16)$ & $89(4)$ \\
\hline$C(33)$ & $4570(20)$ & $1450(20)$ & $3687(13)$ & $89(4)$ \\
\hline$C(34)$ & $3400(20)$ & $-713(17)$ & $2346(19)$ & $81(4)$ \\
\hline$C(35)$ & $10150(20)$ & $4860(30)$ & $8130(20)$ & $108(9)$ \\
\hline$C(36)$ & $11190(20)$ & $5250(30)$ & $8580(20)$ & $109(9)$ \\
\hline$C(37)$ & $9870(30)$ & $6630(30)$ & $8040(20)$ & $110(10)$ \\
\hline$C(38)$ & $10040(30)$ & $6520(30)$ & $7330(20)$ & $111(10)$ \\
\hline$C(39)$ & $9450(30)$ & $5850(20)$ & $9170(20)$ & $117(10)$ \\
\hline $\mathrm{C}(40)$ & $8880(30)$ & $5120(20)$ & $9390(20)$ & $118(10)$ \\
\hline $\mathrm{C}(41)$ & $5890(20)$ & $5710(20)$ & $7928(19)$ & 98(8) \\
\hline$C(42)$ & $6110(30)$ & $5420(20)$ & $8611(18)$ & $98(8)$ \\
\hline$C(43)$ & $7580(30)$ & $7110(20)$ & $8480(20)$ & $130(11)$ \\
\hline $\mathrm{C}(44)$ & $6820(30)$ & $7850(30)$ & $8520(20)$ & 131(11) \\
\hline$C(45)$ & $6620(30)$ & $6610(30)$ & $7048(19)$ & $125(11)$ \\
\hline$C(46)$ & $7230(30)$ & $6950(30)$ & $6770(20)$ & $126(11)$ \\
\hline $\mathrm{C}(47)$ & $1560(20)$ & $-2430(20)$ & 225(19) & $108(10)$ \\
\hline $\mathrm{C}(48)$ & $1450(30)$ & $-2600(20)$ & $927(19)$ & $111(10)$ \\
\hline C(49) & $1930(30)$ & $-510(20)$ & $620(30)$ & $190(20)$ \\
\hline$C(50)$ & $980(30)$ & $-480(30)$ & $60(30)$ & $190(20)$ \\
\hline$C(51)$ & $2440(40)$ & $-1470(30)$ & $-582(17)$ & $184(18)$ \\
\hline$C(52)$ & $3030(40)$ & $-640(30)$ & $-650(30)$ & $186(18)$ \\
\hline$C(53)$ & $3690(40)$ & $-3400(30)$ & $-100(30)$ & $153(13)$ \\
\hline$C(54)$ & $3170(40)$ & $-3980(30)$ & $270(30)$ & $153(13)$ \\
\hline$C(56)$ & $5850(30)$ & $-1170(30)$ & $90(20)$ & $134(11)$ \\
\hline$C(55)$ & $5210(30)$ & $-2060(30)$ & $-240(20)$ & $135(11)$ \\
\hline$C(57)$ & $5500(40)$ & $-2920(30)$ & $880(20)$ & $140(12)$ \\
\hline$C(58)$ & $5840(40)$ & $-3660(30)$ & $430(20)$ & $143(12)$ \\
\hline
\end{tabular}


Table 4. Bond lengths $[\AA]$ and angles $\left[{ }^{\circ}\right]$ for BT2.

\begin{tabular}{|c|c|}
\hline $\mathrm{Pt}(1)-\mathrm{O}(2)$ & $2.052(19)$ \\
\hline $\mathrm{Pt}(1)-\mathrm{N}(3)$ & $2.148(17)$ \\
\hline $\mathrm{Pt}(1)-\mathrm{P}(2)$ & $2.233(7)$ \\
\hline $\mathrm{Pt}(1)-\mathrm{P}(1)$ & $2.258(7)$ \\
\hline $\mathrm{Pt}(2)-\mathrm{O}(3)$ & $2.044(19)$ \\
\hline $\operatorname{Pt}(2)-\mathrm{N}(4)$ & $2.13(2)$ \\
\hline $\mathrm{Pt}(2)-\mathrm{P}(4)$ & $2.253(8)$ \\
\hline $\mathrm{Pt}(2)-\mathrm{P}(3)$ & $2.298(9)$ \\
\hline $\mathrm{Zn}(1)-\mathrm{N}(1) \# 1$ & $2.011(18)$ \\
\hline $\mathrm{Zn}(1)-\mathrm{N}(1)$ & $2.011(18)$ \\
\hline $\mathrm{Zn}(1)-\mathrm{N}(2) \# 1$ & $2.08(2)$ \\
\hline $\mathrm{Zn}(1)-\mathrm{N}(2)$ & $2.08(2)$ \\
\hline $\mathrm{P}(1)-\mathrm{C}(41)$ & $1.77(3)$ \\
\hline$P(1)-C(45)$ & $1.77(3)$ \\
\hline$P(1)-C(43)$ & $1.77(3)$ \\
\hline $\mathrm{P}(2)-\mathrm{C}(39)$ & $1.81(4)$ \\
\hline$P(2)-C(35)$ & $1.82(4)$ \\
\hline $\mathrm{P}(2)-\mathrm{C}(37)$ & $1.87(3)$ \\
\hline $\mathrm{P}(3)-\mathrm{C}(53)$ & $1.78(5)$ \\
\hline$P(3)-C(55)$ & $1.83(4)$ \\
\hline$P(3)-C(57)$ & $1.92(5)$ \\
\hline$P(4)-C(51)$ & $1.83(3)$ \\
\hline $\mathrm{P}(4)-\mathrm{C}(49)$ & $1.83(3)$ \\
\hline$P(4)-C(47)$ & $1.84(2)$ \\
\hline $\mathrm{O}(1)-\mathrm{C}(21)$ & $1.22(3)$ \\
\hline $\mathrm{O}(2)-\mathrm{C}(21)$ & $1.37(4)$ \\
\hline $\mathrm{O}(3)-\mathrm{C}(34)$ & $1.39(4)$ \\
\hline $\mathrm{O}(4)-\mathrm{C}(34)$ & $1.15(3)$ \\
\hline $\mathrm{N}(1)-\mathrm{C}(1)$ & $1.33(3)$ \\
\hline $\mathrm{N}(1)-\mathrm{C}(4)$ & $1.39(3)$ \\
\hline $\mathrm{N}(2)-\mathrm{C}(6)$ & $1.34(3)$ \\
\hline $\mathrm{N}(2)-\mathrm{C}(9)$ & $1.39(3)$ \\
\hline $\mathrm{N}(3)-\mathrm{C}(13)$ & $1.18(3)$ \\
\hline $\mathrm{N}(3)-\mathrm{C}(14)$ & $1.35(3)$ \\
\hline N(4)-C(19) & $1.29(4)$ \\
\hline $\mathrm{N}(4)-\mathrm{C}(18)$ & $1.33(4)$ \\
\hline $\mathrm{C}(1)-\mathrm{C}(2)$ & $1.39(3)$ \\
\hline$C(1)-C(10) \# 1$ & $1.49(3)$ \\
\hline $\mathrm{C}(2)-\mathrm{C}(3)$ & $1.32(3)$ \\
\hline $\mathrm{C}(2)-\mathrm{H}(2)$ & 0.95 \\
\hline
\end{tabular}




\begin{tabular}{|c|c|}
\hline$C(3)-C(4)$ & $1.47(3)$ \\
\hline $\mathrm{C}(3)-\mathrm{H}(3)$ & 0.95 \\
\hline $\mathrm{C}(4)-\mathrm{C}(5)$ & $1.42(3)$ \\
\hline $\mathrm{C}(5)-\mathrm{C}(6)$ & $1.41(4)$ \\
\hline$C(5)-C(11)$ & $1.51(3)$ \\
\hline$C(6)-C(7)$ & $1.47(4)$ \\
\hline $\mathrm{C}(7)-\mathrm{C}(8)$ & $1.43(3)$ \\
\hline $\mathrm{C}(7)-\mathrm{H}(7)$ & 0.95 \\
\hline C(8)-C(9) & $1.44(4)$ \\
\hline $\mathrm{C}(8)-\mathrm{H}(8)$ & 0.95 \\
\hline $\mathrm{C}(9)-\mathrm{C}(10)$ & $1.35(3)$ \\
\hline$C(10)-C(16)$ & $1.55(3)$ \\
\hline $\mathrm{C}(11)-\mathrm{C}(12)$ & $1.31(4)$ \\
\hline$C(11)-C(15)$ & $1.40(3)$ \\
\hline$C(12)-C(13)$ & $1.52(3)$ \\
\hline $\mathrm{C}(12)-\mathrm{H}(12)$ & 0.95 \\
\hline $\mathrm{C}(13)-\mathrm{H}(13)$ & 0.95 \\
\hline$C(14)-C(15)$ & $1.44(3)$ \\
\hline $\mathrm{C}(14)-\mathrm{H}(14)$ & 0.95 \\
\hline $\mathrm{C}(15)-\mathrm{H}(15)$ & 0.95 \\
\hline$C(16)-C(17)$ & $1.34(4)$ \\
\hline$C(16)-C(20)$ & $1.45(4)$ \\
\hline$C(17)-C(18)$ & $1.42(4)$ \\
\hline $\mathrm{C}(17)-\mathrm{H}(17)$ & 0.95 \\
\hline $\mathrm{C}(18)-\mathrm{H}(18)$ & 0.95 \\
\hline$C(19)-C(20)$ & $1.40(4)$ \\
\hline $\mathrm{C}(19)-\mathrm{H}(19)$ & 0.95 \\
\hline $\mathrm{C}(20)-\mathrm{H}(20)$ & 0.95 \\
\hline$C(21)-C(22)$ & $1.53(4)$ \\
\hline$C(22)-C(27)$ & $1.388(18)$ \\
\hline$C(22)-C(23)$ & $1.389(18)$ \\
\hline$C(23)-C(24)$ & $1.386(18)$ \\
\hline $\mathrm{C}(23)-\mathrm{H}(23)$ & 0.95 \\
\hline$C(24)-C(25)$ & $1.384(18)$ \\
\hline $\mathrm{C}(24)-\mathrm{H}(24)$ & 0.95 \\
\hline$C(25)-C(26)$ & $1.389(18)$ \\
\hline$C(25)-C(28)$ & $1.42(4)$ \\
\hline$C(26)-C(27)$ & $1.398(18)$ \\
\hline $\mathrm{C}(26)-\mathrm{H}(26)$ & 0.95 \\
\hline $\mathrm{C}(27)-\mathrm{H}(27)$ & 0.95 \\
\hline$C(28)-C(33)$ & $1.425(18)$ \\
\hline$C(28)-C(29)$ & $1.431(18)$ \\
\hline$C(29)-C(30)$ & $1.418(18)$ \\
\hline
\end{tabular}




\begin{tabular}{|c|c|}
\hline $\mathrm{C}(29)-\mathrm{H}(29)$ & 0.95 \\
\hline $\mathrm{C}(30)-\mathrm{C}(31)$ & $1.424(18)$ \\
\hline $\mathrm{C}(30)-\mathrm{H}(30)$ & 0.95 \\
\hline $\mathrm{C}(31)-\mathrm{C}(32)$ & $1.421(18)$ \\
\hline $\mathrm{C}(31)-\mathrm{C}(34)$ & $1.50(4)$ \\
\hline$C(32)-C(33)$ & $1.417(18)$ \\
\hline $\mathrm{C}(32)-\mathrm{H}(32)$ & 0.95 \\
\hline $\mathrm{C}(33)-\mathrm{H}(33)$ & 0.95 \\
\hline$C(35)-C(36)$ & $1.52(5)$ \\
\hline $\mathrm{C}(35)-\mathrm{H}(35 \mathrm{~A})$ & 0.99 \\
\hline $\mathrm{C}(35)-\mathrm{H}(35 \mathrm{~B})$ & 0.99 \\
\hline $\mathrm{C}(36)-\mathrm{H}(36 \mathrm{~A})$ & 0.98 \\
\hline $\mathrm{C}(36)-\mathrm{H}(36 \mathrm{~B})$ & 0.98 \\
\hline $\mathrm{C}(36)-\mathrm{H}(36 \mathrm{C})$ & 0.98 \\
\hline $\mathrm{C}(37)-\mathrm{C}(38)$ & $1.49(5)$ \\
\hline $\mathrm{C}(37)-\mathrm{H}(37 \mathrm{~A})$ & 0.99 \\
\hline $\mathrm{C}(37)-\mathrm{H}(37 \mathrm{~B})$ & 0.99 \\
\hline $\mathrm{C}(38)-\mathrm{H}(38 \mathrm{~A})$ & 0.98 \\
\hline $\mathrm{C}(38)-\mathrm{H}(38 \mathrm{~B})$ & 0.98 \\
\hline $\mathrm{C}(38)-\mathrm{H}(38 \mathrm{C})$ & 0.98 \\
\hline C(39)-C(40) & $1.53(5)$ \\
\hline $\mathrm{C}(39)-\mathrm{H}(39 \mathrm{~A})$ & 0.99 \\
\hline $\mathrm{C}(39)-\mathrm{H}(39 \mathrm{~B})$ & 0.99 \\
\hline $\mathrm{C}(40)-\mathrm{H}(40 \mathrm{~A})$ & 0.98 \\
\hline $\mathrm{C}(40)-\mathrm{H}(40 \mathrm{~B})$ & 0.98 \\
\hline $\mathrm{C}(40)-\mathrm{H}(40 \mathrm{C})$ & 0.98 \\
\hline $\mathrm{C}(41)-\mathrm{C}(42)$ & $1.50(5)$ \\
\hline $\mathrm{C}(41)-\mathrm{H}(41 \mathrm{~A})$ & 0.99 \\
\hline $\mathrm{C}(41)-\mathrm{H}(41 \mathrm{~B})$ & 0.99 \\
\hline $\mathrm{C}(42)-\mathrm{H}(42 \mathrm{~A})$ & 0.98 \\
\hline $\mathrm{C}(42)-\mathrm{H}(42 \mathrm{~B})$ & 0.98 \\
\hline $\mathrm{C}(42)-\mathrm{H}(42 \mathrm{C})$ & 0.98 \\
\hline$C(43)-C(44)$ & $1.66(5)$ \\
\hline $\mathrm{C}(43)-\mathrm{H}(43 \mathrm{~A})$ & 0.99 \\
\hline $\mathrm{C}(43)-\mathrm{H}(43 \mathrm{~B})$ & 0.99 \\
\hline $\mathrm{C}(44)-\mathrm{H}(44 \mathrm{~A})$ & 0.98 \\
\hline $\mathrm{C}(44)-\mathrm{H}(44 \mathrm{~B})$ & 0.98 \\
\hline $\mathrm{C}(44)-\mathrm{H}(44 \mathrm{C})$ & 0.98 \\
\hline$C(45)-C(46)$ & $1.27(5)$ \\
\hline $\mathrm{C}(45)-\mathrm{H}(45 \mathrm{~A})$ & 0.99 \\
\hline $\mathrm{C}(45)-\mathrm{H}(45 \mathrm{~B})$ & 0.99 \\
\hline $\mathrm{C}(46)-\mathrm{H}(46 \mathrm{~A})$ & 0.98 \\
\hline $\mathrm{C}(46)-\mathrm{H}(46 \mathrm{~B})$ & 0.98 \\
\hline
\end{tabular}




\begin{tabular}{|c|c|}
\hline $\mathrm{C}(46)-\mathrm{H}(46 \mathrm{C})$ & 0.98 \\
\hline C(47)-C(48) & $1.532(10)$ \\
\hline $\mathrm{C}(47)-\mathrm{H}(47 \mathrm{~A})$ & 0.99 \\
\hline C(47)-H(47B) & 0.99 \\
\hline $\mathrm{C}(48)-\mathrm{H}(48 \mathrm{~A})$ & 0.98 \\
\hline C(48)-H(48B) & 0.98 \\
\hline $\mathrm{C}(48)-\mathrm{H}(48 \mathrm{C})$ & 0.98 \\
\hline$C(49)-C(50)$ & $1.534(10)$ \\
\hline C(49)-H(49A) & 0.99 \\
\hline C(49)-H(49B) & 0.99 \\
\hline $\mathrm{C}(50)-\mathrm{H}(50 \mathrm{~A})$ & 0.98 \\
\hline $\mathrm{C}(50)-\mathrm{H}(50 \mathrm{~B})$ & 0.98 \\
\hline $\mathrm{C}(50)-\mathrm{H}(50 \mathrm{C})$ & 0.98 \\
\hline $\mathrm{C}(51)-\mathrm{C}(52)$ & $1.531(10)$ \\
\hline $\mathrm{C}(51)-\mathrm{H}(51 \mathrm{~A})$ & 0.99 \\
\hline $\mathrm{C}(51)-\mathrm{H}(51 \mathrm{~B})$ & 0.99 \\
\hline $\mathrm{C}(52)-\mathrm{H}(52 \mathrm{~A})$ & 0.98 \\
\hline C(52)-H(52B) & 0.98 \\
\hline $\mathrm{C}(52)-\mathrm{H}(52 \mathrm{C})$ & 0.98 \\
\hline$C(53)-C(54)$ & $1.529(10)$ \\
\hline $\mathrm{C}(53)-\mathrm{H}(53 \mathrm{~A})$ & 0.99 \\
\hline C(53)-H(53B) & 0.99 \\
\hline $\mathrm{C}(54)-\mathrm{H}(54 \mathrm{~A})$ & 0.98 \\
\hline C(54)-H(54B) & 0.98 \\
\hline $\mathrm{C}(54)-\mathrm{H}(54 \mathrm{C})$ & 0.98 \\
\hline $\mathrm{C}(56)-\mathrm{C}(55)$ & $1.530(10)$ \\
\hline $\mathrm{C}(56)-\mathrm{H}(56 \mathrm{~A})$ & 0.98 \\
\hline $\mathrm{C}(56)-\mathrm{H}(56 \mathrm{~B})$ & 0.98 \\
\hline $\mathrm{C}(56)-\mathrm{H}(56 \mathrm{C})$ & 0.98 \\
\hline $\mathrm{C}(55)-\mathrm{H}(55 \mathrm{~A})$ & 0.99 \\
\hline $\mathrm{C}(55)-\mathrm{H}(55 \mathrm{~B})$ & 0.99 \\
\hline $\mathrm{C}(57)-\mathrm{C}(58)$ & $1.531(10)$ \\
\hline $\mathrm{C}(57)-\mathrm{H}(57 \mathrm{~A})$ & 0.99 \\
\hline C(57)-H(57B) & 0.99 \\
\hline $\mathrm{C}(58)-\mathrm{H}(58 \mathrm{~A})$ & 0.98 \\
\hline C(58)-H(58B) & 0.98 \\
\hline $\mathrm{C}(58)-\mathrm{H}(58 \mathrm{C})$ & 0.98 \\
\hline $\mathrm{O}(2)-\mathrm{Pt}(1)-\mathrm{N}(3)$ & $84.8(7)$ \\
\hline $\mathrm{O}(2)-\mathrm{Pt}(1)-\mathrm{P}(2)$ & $176.2(6)$ \\
\hline $\mathrm{N}(3)-\operatorname{Pt}(1)-\mathrm{P}(2)$ & $94.5(6)$ \\
\hline $\mathrm{O}(2)-\mathrm{Pt}(1)-\mathrm{P}(1)$ & $82.8(5)$ \\
\hline $\mathrm{N}(3)-\operatorname{Pt}(1)-\mathrm{P}(1)$ & $167.0(6)$ \\
\hline
\end{tabular}




\begin{tabular}{|c|c|}
\hline $\mathrm{P}(2)-\mathrm{Pt}(1)-\mathrm{P}(1)$ & $97.6(3)$ \\
\hline $\mathrm{O}(3)-\mathrm{Pt}(2)-\mathrm{N}(4)$ & $81.6(8)$ \\
\hline $\mathrm{O}(3)-\mathrm{Pt}(2)-\mathrm{P}(4)$ & $88.4(6)$ \\
\hline N(4)-Pt(2)-P(4) & $170.0(6)$ \\
\hline $\mathrm{O}(3)-\mathrm{Pt}(2)-\mathrm{P}(3)$ & $172.3(5)$ \\
\hline $\mathrm{N}(4)-\operatorname{Pt}(2)-\mathrm{P}(3)$ & $95.7(6)$ \\
\hline $\mathrm{P}(4)-\mathrm{Pt}(2)-\mathrm{P}(3)$ & $94.0(3)$ \\
\hline N(1)\#1-Zn(1)-N(1) & 180 \\
\hline $\mathrm{N}(1) \# 1-\mathrm{Zn}(1)-\mathrm{N}(2) \# 1$ & $89.8(8)$ \\
\hline $\mathrm{N}(1)-\mathrm{Zn}(1)-\mathrm{N}(2) \# 1$ & $90.2(8)$ \\
\hline $\mathrm{N}(1) \# 1-\mathrm{Zn}(1)-\mathrm{N}(2)$ & $90.2(8)$ \\
\hline $\mathrm{N}(1)-\mathrm{Zn}(1)-\mathrm{N}(2)$ & $89.8(8)$ \\
\hline $\mathrm{N}(2) \# 1-\mathrm{Zn}(1)-\mathrm{N}(2)$ & 180 \\
\hline C(41)-P(1)-C(45) & $104(2)$ \\
\hline $\mathrm{C}(41)-\mathrm{P}(1)-\mathrm{C}(43)$ & $112(2)$ \\
\hline $\mathrm{C}(45)-\mathrm{P}(1)-\mathrm{C}(43)$ & $104(2)$ \\
\hline C(41)-P(1)-Pt(1) & $105.1(13)$ \\
\hline $\mathrm{C}(45)-\mathrm{P}(1)-\mathrm{Pt}(1)$ & $107.3(15)$ \\
\hline C(43)-P(1)-Pt(1) & $122.1(15)$ \\
\hline C(39)-P(2)-C(35) & $101(2)$ \\
\hline $\mathrm{C}(39)-\mathrm{P}(2)-\mathrm{C}(37)$ & $105.9(19)$ \\
\hline C(35)-P(2)-C(37) & $103.1(18)$ \\
\hline C(39)-P(2)-Pt(1) & $119.1(15)$ \\
\hline $\mathrm{C}(35)-\mathrm{P}(2)-\mathrm{Pt}(1)$ & $110.2(13)$ \\
\hline $\mathrm{C}(37)-\mathrm{P}(2)-\mathrm{Pt}(1)$ & $115.8(13)$ \\
\hline C(53)-P(3)-C(55) & $111(2)$ \\
\hline $\mathrm{C}(53)-\mathrm{P}(3)-\mathrm{C}(57)$ & $97(2)$ \\
\hline $\mathrm{C}(55)-\mathrm{P}(3)-\mathrm{C}(57)$ & $97(2)$ \\
\hline $\mathrm{C}(53)-\mathrm{P}(3)-\mathrm{Pt}(2)$ & $116.9(17)$ \\
\hline $\mathrm{C}(55)-\mathrm{P}(3)-\mathrm{Pt}(2)$ & $122.5(14)$ \\
\hline C(57)-P(3)-Pt(2) & $107.2(12)$ \\
\hline C(51)-P(4)-C(49) & $104(3)$ \\
\hline $\mathrm{C}(51)-\mathrm{P}(4)-\mathrm{C}(47)$ & $101.3(18)$ \\
\hline C(49)-P(4)-C(47) & $106(2)$ \\
\hline $\mathrm{C}(51)-\mathrm{P}(4)-\mathrm{Pt}(2)$ & $119(2)$ \\
\hline C(49)-P(4)-Pt(2) & $109.7(11)$ \\
\hline C(47)-P(4)-Pt(2) & $115.6(13)$ \\
\hline $\mathrm{C}(21)-\mathrm{O}(2)-\mathrm{Pt}(1)$ & $110.0(18)$ \\
\hline $\mathrm{C}(34)-\mathrm{O}(3)-\mathrm{Pt}(2)$ & $111.9(16)$ \\
\hline C(1)-N(1)-C(4) & $103.6(18)$ \\
\hline $\mathrm{C}(1)-\mathrm{N}(1)-\mathrm{Zn}(1)$ & $129.8(15)$ \\
\hline $\mathrm{C}(4)-\mathrm{N}(1)-\mathrm{Zn}(1)$ & $126.6(17)$ \\
\hline $\mathrm{C}(6)-\mathrm{N}(2)-\mathrm{C}(9)$ & $108(2)$ \\
\hline
\end{tabular}




\begin{tabular}{|c|c|}
\hline $\mathrm{C}(6)-\mathrm{N}(2)-\mathrm{Zn}(1)$ & $127.6(18)$ \\
\hline $\mathrm{C}(9)-\mathrm{N}(2)-\mathrm{Zn}(1)$ & $124.3(16)$ \\
\hline $\mathrm{C}(13)-\mathrm{N}(3)-\mathrm{C}(14)$ & $126(2)$ \\
\hline C(13)-N(3)-Pt(1) & $115.5(16)$ \\
\hline $\mathrm{C}(14)-\mathrm{N}(3)-\mathrm{Pt}(1)$ & $118.2(16)$ \\
\hline C(19)-N(4)-C(18) & $120(2)$ \\
\hline C(19)-N(4)-Pt(2) & $122.1(19)$ \\
\hline $\mathrm{C}(18)-\mathrm{N}(4)-\mathrm{Pt}(2)$ & $116(2)$ \\
\hline $\mathrm{N}(1)-\mathrm{C}(1)-\mathrm{C}(2)$ & $113(2)$ \\
\hline $\mathrm{N}(1)-\mathrm{C}(1)-\mathrm{C}(10) \# 1$ & $123(2)$ \\
\hline$C(2)-C(1)-C(10) \# 1$ & $124(2)$ \\
\hline $\mathrm{C}(3)-\mathrm{C}(2)-\mathrm{C}(1)$ & $109(2)$ \\
\hline $\mathrm{C}(3)-\mathrm{C}(2)-\mathrm{H}(2)$ & 125.7 \\
\hline $\mathrm{C}(1)-\mathrm{C}(2)-\mathrm{H}(2)$ & 125.7 \\
\hline$C(2)-C(3)-C(4)$ & $105(2)$ \\
\hline $\mathrm{C}(2)-\mathrm{C}(3)-\mathrm{H}(3)$ & 127.6 \\
\hline $\mathrm{C}(4)-\mathrm{C}(3)-\mathrm{H}(3)$ & 127.6 \\
\hline $\mathrm{N}(1)-\mathrm{C}(4)-\mathrm{C}(5)$ & $126(2)$ \\
\hline $\mathrm{N}(1)-\mathrm{C}(4)-\mathrm{C}(3)$ & $110(2)$ \\
\hline$C(5)-C(4)-C(3)$ & $124(2)$ \\
\hline$C(6)-C(5)-C(4)$ & $124(2)$ \\
\hline $\mathrm{C}(6)-\mathrm{C}(5)-\mathrm{C}(11)$ & $118(2)$ \\
\hline$C(4)-C(5)-C(11)$ & $117(2)$ \\
\hline $\mathrm{N}(2)-\mathrm{C}(6)-\mathrm{C}(5)$ & $125(2)$ \\
\hline $\mathrm{N}(2)-\mathrm{C}(6)-\mathrm{C}(7)$ & $110(2)$ \\
\hline$C(5)-C(6)-C(7)$ & $125(2)$ \\
\hline$C(8)-C(7)-C(6)$ & $106(2)$ \\
\hline $\mathrm{C}(8)-\mathrm{C}(7)-\mathrm{H}(7)$ & 126.9 \\
\hline $\mathrm{C}(6)-\mathrm{C}(7)-\mathrm{H}(7)$ & 126.9 \\
\hline $\mathrm{C}(7)-\mathrm{C}(8)-\mathrm{C}(9)$ & $105(2)$ \\
\hline $\mathrm{C}(7)-\mathrm{C}(8)-\mathrm{H}(8)$ & 127.7 \\
\hline $\mathrm{C}(9)-\mathrm{C}(8)-\mathrm{H}(8)$ & 127.7 \\
\hline $\mathrm{C}(10)-\mathrm{C}(9)-\mathrm{N}(2)$ & $127(2)$ \\
\hline $\mathrm{C}(10)-\mathrm{C}(9)-\mathrm{C}(8)$ & $122(2)$ \\
\hline $\mathrm{N}(2)-\mathrm{C}(9)-\mathrm{C}(8)$ & $111(2)$ \\
\hline$C(9)-C(10)-C(1) \# 1$ & $125(2)$ \\
\hline $\mathrm{C}(9)-\mathrm{C}(10)-\mathrm{C}(16)$ & $122(2)$ \\
\hline $\mathrm{C}(1) \# 1-\mathrm{C}(10)-\mathrm{C}(16)$ & $111.6(19)$ \\
\hline $\mathrm{C}(12)-\mathrm{C}(11)-\mathrm{C}(15)$ & $122(2)$ \\
\hline $\mathrm{C}(12)-\mathrm{C}(11)-\mathrm{C}(5)$ & $119(3)$ \\
\hline $\mathrm{C}(15)-\mathrm{C}(11)-\mathrm{C}(5)$ & $119(3)$ \\
\hline$C(11)-C(12)-C(13)$ & $117(3)$ \\
\hline $\mathrm{C}(11)-\mathrm{C}(12)-\mathrm{H}(12)$ & 121.7 \\
\hline
\end{tabular}




$\begin{array}{ll}\mathrm{C}(13)-\mathrm{C}(12)-\mathrm{H}(12) & 121.7 \\ \mathrm{~N}(3)-\mathrm{C}(13)-\mathrm{C}(12) & 121(3) \\ \mathrm{N}(3)-\mathrm{C}(13)-\mathrm{H}(13) & 119.7 \\ \mathrm{C}(12)-\mathrm{C}(13)-\mathrm{H}(13) & 119.7 \\ \mathrm{~N}(3)-\mathrm{C}(14)-\mathrm{C}(15) & 118(2) \\ \mathrm{N}(3)-\mathrm{C}(14)-\mathrm{H}(14) & 121 \\ \mathrm{C}(15)-\mathrm{C}(14)-\mathrm{H}(14) & 121 \\ \mathrm{C}(11)-\mathrm{C}(15)-\mathrm{C}(14) & 117(2) \\ \mathrm{C}(11)-\mathrm{C}(15)-\mathrm{H}(15) & 121.5 \\ \mathrm{C}(14)-\mathrm{C}(15)-\mathrm{H}(15) & 121.5 \\ \mathrm{C}(17)-\mathrm{C}(16)-\mathrm{C}(20) & 118(2) \\ \mathrm{C}(17)-\mathrm{C}(16)-\mathrm{C}(10) & 124(2) \\ \mathrm{C}(20)-\mathrm{C}(16)-\mathrm{C}(10) & 117(2) \\ \mathrm{C}(16)-\mathrm{C}(17)-\mathrm{C}(18) & 120(3) \\ \mathrm{C}(16)-\mathrm{C}(17)-\mathrm{H}(17) & 120 \\ \mathrm{C}(18)-\mathrm{C}(17)-\mathrm{H}(17) & 120 \\ \mathrm{~N}(4)-\mathrm{C}(18)-\mathrm{C}(17) & 121(3) \\ \mathrm{N}(4)-\mathrm{C}(18)-\mathrm{H}(18) & 119.7 \\ \mathrm{C}(17)-\mathrm{C}(18)-\mathrm{H}(18) & 119.7 \\ \mathrm{~N}(4)-\mathrm{C}(19)-\mathrm{C}(20) & 123(3) \\ \mathrm{N}(4)-\mathrm{C}(19)-\mathrm{H}(19) & 118.3 \\ \mathrm{C}(20)-\mathrm{C}(19)-\mathrm{H}(19) & 118.3 \\ \mathrm{C}(19)-\mathrm{C}(20)-\mathrm{C}(16) & 117(3) \\ \mathrm{C}(19)-\mathrm{C}(20)-\mathrm{H}(20) & 121.7 \\ \mathrm{C}(16)-\mathrm{C}(20)-\mathrm{H}(20) & 121.7 \\ \mathrm{O}(1)-\mathrm{C}(21)-\mathrm{O}(2) & 132(3) \\ \mathrm{O}(1)-\mathrm{C}(21)-\mathrm{C}(22) & 114(3) \\ \mathrm{O}(2)-\mathrm{C}(21)-\mathrm{C}(22) & 113(3) \\ \mathrm{C}(27)-\mathrm{C}(22)-\mathrm{C}(23) & 119(3) \\ \mathrm{C}(27)-\mathrm{C}(22)-\mathrm{C}(21) & 122(2) \\ \mathrm{C}(23)-\mathrm{C}(22)-\mathrm{C}(21) & 119(2) \\ \mathrm{C}(24)-\mathrm{C}(23)-\mathrm{C}(22) & 118(3) \\ \mathrm{C}(24)-\mathrm{C}(23)-\mathrm{H}(23) & 120.9 \\ \mathrm{C}(22)-\mathrm{C}(23)-\mathrm{H}(23) & 120.9 \\ \mathrm{C}(25)-\mathrm{C}(24)-\mathrm{C}(23) & 125(3) \\ \mathrm{C}(25)-\mathrm{C}(24)-\mathrm{H}(24) & 117.7 \\ \mathrm{C}(23)-\mathrm{C}(24)-\mathrm{H}(24) & 117.7 \\ \mathrm{C}(24)-\mathrm{C}(25)-\mathrm{C}(26) & 116(3) \\ \mathrm{C}(25)-\mathrm{C}(25)-\mathrm{C}(28)-\mathrm{C}(28)-\mathrm{C}(27) & \\ \mathrm{C}(27)-\mathrm{C}(26)-\mathrm{H}(26) & \\ & \end{array}$




$\begin{array}{ll}\mathrm{C}(22)-\mathrm{C}(27)-\mathrm{C}(26) & 122(3) \\ \mathrm{C}(22)-\mathrm{C}(27)-\mathrm{H}(27) & 119.2 \\ \mathrm{C}(26)-\mathrm{C}(27)-\mathrm{H}(27) & 119.2 \\ \mathrm{C}(25)-\mathrm{C}(28)-\mathrm{C}(33) & 121(2) \\ \mathrm{C}(25)-\mathrm{C}(28)-\mathrm{C}(29) & 121(2) \\ \mathrm{C}(33)-\mathrm{C}(28)-\mathrm{C}(29) & 117(3) \\ \mathrm{C}(30)-\mathrm{C}(29)-\mathrm{C}(28) & 121(3) \\ \mathrm{C}(30)-\mathrm{C}(29)-\mathrm{H}(29) & 119.4 \\ \mathrm{C}(28)-\mathrm{C}(29)-\mathrm{H}(29) & 119.4 \\ \mathrm{C}(29)-\mathrm{C}(30)-\mathrm{C}(31) & 122(3) \\ \mathrm{C}(29)-\mathrm{C}(30)-\mathrm{H}(30) & 119.1 \\ \mathrm{C}(31)-\mathrm{C}(30)-\mathrm{H}(30) & 119.1 \\ \mathrm{C}(32)-\mathrm{C}(31)-\mathrm{C}(30) & 117(3) \\ \mathrm{C}(32)-\mathrm{C}(31)-\mathrm{C}(34) & 123(2) \\ \mathrm{C}(30)-\mathrm{C}(31)-\mathrm{C}(34) & 119(2) \\ \mathrm{C}(33)-\mathrm{C}(32)-\mathrm{C}(31) & 121(3) \\ \mathrm{C}(33)-\mathrm{C}(32)-\mathrm{H}(32) & 119.3 \\ \mathrm{C}(31)-\mathrm{C}(32)-\mathrm{H}(32) & 119.3 \\ \mathrm{C}(32)-\mathrm{C}(33)-\mathrm{C}(28) & 122(3) \\ \mathrm{C}(32)-\mathrm{C}(33)-\mathrm{H}(33) & 119.1 \\ \mathrm{C}(28)-\mathrm{C}(33)-\mathrm{H}(33) & 119.1 \\ \mathrm{O}(4)-\mathrm{C}(34)-\mathrm{O}(3) & 126(3) \\ \mathrm{O}(4)-\mathrm{C}(34)-\mathrm{C}(31) & 121(3) \\ \mathrm{O}(3)-\mathrm{C}(34)-\mathrm{C}(31) & 113(2) \\ \mathrm{C}(36)-\mathrm{C}(35)-\mathrm{P}(2) & 112(3) \\ \mathrm{C}(36)-\mathrm{C}(35)-\mathrm{H}(35 \mathrm{~A}) & 109.3 \\ \mathrm{P}(2)-\mathrm{C}(35)-\mathrm{H}(35 \mathrm{~A}) & 109.3 \\ \mathrm{C}(36)-\mathrm{C}(35)-\mathrm{H}(35 \mathrm{~B}) & 109.3 \\ \mathrm{P}(2)-\mathrm{C}(35)-\mathrm{H}(35 \mathrm{~B}) & 109.3 \\ \mathrm{H}(35 \mathrm{~A})-\mathrm{C}(35)-\mathrm{H}(35 \mathrm{~B}) & 108 \\ \mathrm{C}(35)-\mathrm{C}(36)-\mathrm{H}(36 \mathrm{~A}) & 109.5 \\ \mathrm{C}(35)-\mathrm{C}(36)-\mathrm{H}(36 \mathrm{~B}) & 109.5 \\ \mathrm{H}(36 \mathrm{~A})-\mathrm{C}(36)-\mathrm{H}(36 \mathrm{~B}) & 109.5 \\ \mathrm{C}(35)-\mathrm{C}(36)-\mathrm{H}(36 \mathrm{C}) & 109.5 \\ \mathrm{H}(36 \mathrm{~A})-\mathrm{C}(36)-\mathrm{H}(36 \mathrm{C}) & 109.5 \\ \mathrm{H}(36 \mathrm{~B})-\mathrm{C}(36)-\mathrm{H}(36 \mathrm{C}) & 109.5 \\ \mathrm{C}(38)-\mathrm{C}(37)-\mathrm{P}(2) & 115(3) \\ \mathrm{C}(38)-\mathrm{C}(37)-\mathrm{H}(37 \mathrm{~A}) & 108.6 \\ & \end{array}$




\begin{tabular}{|c|c|}
\hline $\mathrm{C}(37)-\mathrm{C}(38)-\mathrm{H}(38 \mathrm{~B})$ & 109.5 \\
\hline $\mathrm{H}(38 \mathrm{~A})-\mathrm{C}(38)-\mathrm{H}(38 \mathrm{~B})$ & 109.5 \\
\hline C(37)-C(38)-H(38C) & 109.5 \\
\hline $\mathrm{H}(38 \mathrm{~A})-\mathrm{C}(38)-\mathrm{H}(38 \mathrm{C})$ & 109.5 \\
\hline $\mathrm{H}(38 \mathrm{~B})-\mathrm{C}(38)-\mathrm{H}(38 \mathrm{C})$ & 109.5 \\
\hline $\mathrm{C}(40)-\mathrm{C}(39)-\mathrm{P}(2)$ & $112(2)$ \\
\hline $\mathrm{C}(40)-\mathrm{C}(39)-\mathrm{H}(39 \mathrm{~A})$ & 109.3 \\
\hline P(2)-C(39)-H(39A) & 109.3 \\
\hline C(40)-C(39)-H(39B) & 109.3 \\
\hline P(2)-C(39)-H(39B) & 109.3 \\
\hline $\mathrm{H}(39 \mathrm{~A})-\mathrm{C}(39)-\mathrm{H}(39 \mathrm{~B})$ & 107.9 \\
\hline $\mathrm{C}(39)-\mathrm{C}(40)-\mathrm{H}(40 \mathrm{~A})$ & 109.5 \\
\hline $\mathrm{C}(39)-\mathrm{C}(40)-\mathrm{H}(40 \mathrm{~B})$ & 109.5 \\
\hline $\mathrm{H}(40 \mathrm{~A})-\mathrm{C}(40)-\mathrm{H}(40 \mathrm{~B})$ & 109.5 \\
\hline C(39)-C(40)-H(40C) & 109.5 \\
\hline $\mathrm{H}(40 \mathrm{~A})-\mathrm{C}(40)-\mathrm{H}(40 \mathrm{C})$ & 109.5 \\
\hline $\mathrm{H}(40 \mathrm{~B})-\mathrm{C}(40)-\mathrm{H}(40 \mathrm{C})$ & 109.5 \\
\hline $\mathrm{C}(42)-\mathrm{C}(41)-\mathrm{P}(1)$ & $109(2)$ \\
\hline $\mathrm{C}(42)-\mathrm{C}(41)-\mathrm{H}(41 \mathrm{~A})$ & 109.8 \\
\hline $\mathrm{P}(1)-\mathrm{C}(41)-\mathrm{H}(41 \mathrm{~A})$ & 109.8 \\
\hline C(42)-C(41)-H(41B) & 109.8 \\
\hline $\mathrm{P}(1)-\mathrm{C}(41)-\mathrm{H}(41 \mathrm{~B})$ & 109.8 \\
\hline $\mathrm{H}(41 \mathrm{~A})-\mathrm{C}(41)-\mathrm{H}(41 \mathrm{~B})$ & 108.3 \\
\hline $\mathrm{C}(41)-\mathrm{C}(42)-\mathrm{H}(42 \mathrm{~A})$ & 109.5 \\
\hline $\mathrm{C}(41)-\mathrm{C}(42)-\mathrm{H}(42 \mathrm{~B})$ & 109.5 \\
\hline $\mathrm{H}(42 \mathrm{~A})-\mathrm{C}(42)-\mathrm{H}(42 \mathrm{~B})$ & 109.5 \\
\hline $\mathrm{C}(41)-\mathrm{C}(42)-\mathrm{H}(42 \mathrm{C})$ & 109.5 \\
\hline $\mathrm{H}(42 \mathrm{~A})-\mathrm{C}(42)-\mathrm{H}(42 \mathrm{C})$ & 109.5 \\
\hline $\mathrm{H}(42 \mathrm{~B})-\mathrm{C}(42)-\mathrm{H}(42 \mathrm{C})$ & 109.5 \\
\hline $\mathrm{C}(44)-\mathrm{C}(43)-\mathrm{P}(1)$ & $110(3)$ \\
\hline $\mathrm{C}(44)-\mathrm{C}(43)-\mathrm{H}(43 \mathrm{~A})$ & 109.7 \\
\hline $\mathrm{P}(1)-\mathrm{C}(43)-\mathrm{H}(43 \mathrm{~A})$ & 109.7 \\
\hline $\mathrm{C}(44)-\mathrm{C}(43)-\mathrm{H}(43 \mathrm{~B})$ & 109.7 \\
\hline $\mathrm{P}(1)-\mathrm{C}(43)-\mathrm{H}(43 \mathrm{~B})$ & 109.7 \\
\hline $\mathrm{H}(43 \mathrm{~A})-\mathrm{C}(43)-\mathrm{H}(43 \mathrm{~B})$ & 108.2 \\
\hline $\mathrm{C}(43)-\mathrm{C}(44)-\mathrm{H}(44 \mathrm{~A})$ & 109.5 \\
\hline C(43)-C(44)-H(44B) & 109.5 \\
\hline $\mathrm{H}(44 \mathrm{~A})-\mathrm{C}(44)-\mathrm{H}(44 \mathrm{~B})$ & 109.5 \\
\hline $\mathrm{C}(43)-\mathrm{C}(44)-\mathrm{H}(44 \mathrm{C})$ & 109.5 \\
\hline $\mathrm{H}(44 \mathrm{~A})-\mathrm{C}(44)-\mathrm{H}(44 \mathrm{C})$ & 109.5 \\
\hline $\mathrm{H}(44 \mathrm{~B})-\mathrm{C}(44)-\mathrm{H}(44 \mathrm{C})$ & 109.5 \\
\hline $\mathrm{C}(46)-\mathrm{C}(45)-\mathrm{P}(1)$ & $122(4)$ \\
\hline $\mathrm{C}(46)-\mathrm{C}(45)-\mathrm{H}(45 \mathrm{~A})$ & 106.8 \\
\hline
\end{tabular}




\begin{tabular}{|c|c|}
\hline $\mathrm{P}(1)-\mathrm{C}(45)-\mathrm{H}(45 \mathrm{~A})$ & 106.8 \\
\hline $\mathrm{C}(46)-\mathrm{C}(45)-\mathrm{H}(45 \mathrm{~B})$ & 106.8 \\
\hline $\mathrm{P}(1)-\mathrm{C}(45)-\mathrm{H}(45 \mathrm{~B})$ & 106.8 \\
\hline $\mathrm{H}(45 \mathrm{~A})-\mathrm{C}(45)-\mathrm{H}(45 \mathrm{~B})$ & 106.6 \\
\hline C(45)-C(46)-H(46A) & 109.5 \\
\hline $\mathrm{C}(45)-\mathrm{C}(46)-\mathrm{H}(46 \mathrm{~B})$ & 109.5 \\
\hline $\mathrm{H}(46 \mathrm{~A})-\mathrm{C}(46)-\mathrm{H}(46 \mathrm{~B})$ & 109.5 \\
\hline C(45)-C(46)-H(46C) & 109.5 \\
\hline $\mathrm{H}(46 \mathrm{~A})-\mathrm{C}(46)-\mathrm{H}(46 \mathrm{C})$ & 109.5 \\
\hline $\mathrm{H}(46 \mathrm{~B})-\mathrm{C}(46)-\mathrm{H}(46 \mathrm{C})$ & 109.5 \\
\hline $\mathrm{C}(48)-\mathrm{C}(47)-\mathrm{P}(4)$ & $113(2)$ \\
\hline $\mathrm{C}(48)-\mathrm{C}(47)-\mathrm{H}(47 \mathrm{~A})$ & 109.1 \\
\hline $\mathrm{P}(4)-\mathrm{C}(47)-\mathrm{H}(47 \mathrm{~A})$ & 109.1 \\
\hline $\mathrm{C}(48)-\mathrm{C}(47)-\mathrm{H}(47 \mathrm{~B})$ & 109.1 \\
\hline $\mathrm{P}(4)-\mathrm{C}(47)-\mathrm{H}(47 \mathrm{~B})$ & 109.1 \\
\hline $\mathrm{H}(47 \mathrm{~A})-\mathrm{C}(47)-\mathrm{H}(47 \mathrm{~B})$ & 107.8 \\
\hline $\mathrm{C}(47)-\mathrm{C}(48)-\mathrm{H}(48 \mathrm{~A})$ & 109.5 \\
\hline $\mathrm{C}(47)-\mathrm{C}(48)-\mathrm{H}(48 \mathrm{~B})$ & 109.5 \\
\hline $\mathrm{H}(48 \mathrm{~A})-\mathrm{C}(48)-\mathrm{H}(48 \mathrm{~B})$ & 109.5 \\
\hline $\mathrm{C}(47)-\mathrm{C}(48)-\mathrm{H}(48 \mathrm{C})$ & 109.5 \\
\hline $\mathrm{H}(48 \mathrm{~A})-\mathrm{C}(48)-\mathrm{H}(48 \mathrm{C})$ & 109.5 \\
\hline $\mathrm{H}(48 \mathrm{~B})-\mathrm{C}(48)-\mathrm{H}(48 \mathrm{C})$ & 109.5 \\
\hline$C(50)-C(49)-P(4)$ & $110(3)$ \\
\hline $\mathrm{C}(50)-\mathrm{C}(49)-\mathrm{H}(49 \mathrm{~A})$ & 109.6 \\
\hline $\mathrm{P}(4)-\mathrm{C}(49)-\mathrm{H}(49 \mathrm{~A})$ & 109.6 \\
\hline C(50)-C(49)-H(49B) & 109.6 \\
\hline P(4)-C(49)-H(49B) & 109.6 \\
\hline H(49A)-C(49)-H(49B) & 108.1 \\
\hline $\mathrm{C}(49)-\mathrm{C}(50)-\mathrm{H}(50 \mathrm{~A})$ & 109.5 \\
\hline $\mathrm{C}(49)-\mathrm{C}(50)-\mathrm{H}(50 \mathrm{~B})$ & 109.5 \\
\hline $\mathrm{H}(50 \mathrm{~A})-\mathrm{C}(50)-\mathrm{H}(50 \mathrm{~B})$ & 109.5 \\
\hline $\mathrm{C}(49)-\mathrm{C}(50)-\mathrm{H}(50 \mathrm{C})$ & 109.5 \\
\hline $\mathrm{H}(50 \mathrm{~A})-\mathrm{C}(50)-\mathrm{H}(50 \mathrm{C})$ & 109.5 \\
\hline $\mathrm{H}(50 \mathrm{~B})-\mathrm{C}(50)-\mathrm{H}(50 \mathrm{C})$ & 109.5 \\
\hline $\mathrm{C}(52)-\mathrm{C}(51)-\mathrm{P}(4)$ & $112(3)$ \\
\hline $\mathrm{C}(52)-\mathrm{C}(51)-\mathrm{H}(51 \mathrm{~A})$ & 109.2 \\
\hline $\mathrm{P}(4)-\mathrm{C}(51)-\mathrm{H}(51 \mathrm{~A})$ & 109.2 \\
\hline C(52)-C(51)-H(51B) & 109.2 \\
\hline $\mathrm{P}(4)-\mathrm{C}(51)-\mathrm{H}(51 \mathrm{~B})$ & 109.2 \\
\hline $\mathrm{H}(51 \mathrm{~A})-\mathrm{C}(51)-\mathrm{H}(51 \mathrm{~B})$ & 107.9 \\
\hline $\mathrm{C}(51)-\mathrm{C}(52)-\mathrm{H}(52 \mathrm{~A})$ & 109.5 \\
\hline $\mathrm{C}(51)-\mathrm{C}(52)-\mathrm{H}(52 \mathrm{~B})$ & 109.5 \\
\hline $\mathrm{H}(52 \mathrm{~A})-\mathrm{C}(52)-\mathrm{H}(52 \mathrm{~B})$ & 109.5 \\
\hline
\end{tabular}




\begin{tabular}{ll}
$\mathrm{C}(51)-\mathrm{C}(52)-\mathrm{H}(52 \mathrm{C})$ & 109.5 \\
$\mathrm{H}(52 \mathrm{~A})-\mathrm{C}(52)-\mathrm{H}(52 \mathrm{C})$ & 109.5 \\
$\mathrm{H}(52 \mathrm{~B})-\mathrm{C}(52)-\mathrm{H}(52 \mathrm{C})$ & 109.5 \\
$\mathrm{C}(54)-\mathrm{C}(53)-\mathrm{P}(3)$ & $124(4)$ \\
$\mathrm{C}(54)-\mathrm{C}(53)-\mathrm{H}(53 \mathrm{~A})$ & 106.3 \\
$\mathrm{P}(3)-\mathrm{C}(53)-\mathrm{H}(53 \mathrm{~A})$ & 106.3 \\
$\mathrm{C}(54)-\mathrm{C}(53)-\mathrm{H}(53 \mathrm{~B})$ & 106.3 \\
$\mathrm{P}(3)-\mathrm{C}(53)-\mathrm{H}(53 \mathrm{~B})$ & 106.3 \\
$\mathrm{H}(53 \mathrm{~A})-\mathrm{C}(53)-\mathrm{H}(53 \mathrm{~B})$ & 106.4 \\
$\mathrm{C}(53)-\mathrm{C}(54)-\mathrm{H}(54 \mathrm{~A})$ & 109.5 \\
$\mathrm{C}(53)-\mathrm{C}(54)-\mathrm{H}(54 \mathrm{~B})$ & 109.5 \\
$\mathrm{H}(54 \mathrm{~A})-\mathrm{C}(54)-\mathrm{H}(54 \mathrm{~B})$ & 109.5 \\
$\mathrm{C}(53)-\mathrm{C}(54)-\mathrm{H}(54 \mathrm{C})$ & 109.5 \\
$\mathrm{H}(54 \mathrm{~A})-\mathrm{C}(54)-\mathrm{H}(54 \mathrm{C})$ & 109.5 \\
$\mathrm{H}(54 \mathrm{~B})-\mathrm{C}(54)-\mathrm{H}(54 \mathrm{C})$ & 109.5 \\
$\mathrm{C}(55)-\mathrm{C}(56)-\mathrm{H}(56 \mathrm{~A})$ & 109.5 \\
$\mathrm{C}(55)-\mathrm{C}(56)-\mathrm{H}(56 \mathrm{~B})$ & 109.5 \\
$\mathrm{H}(56 \mathrm{~A})-\mathrm{C}(56)-\mathrm{H}(56 \mathrm{~B})$ & 109.5 \\
$\mathrm{C}(55)-\mathrm{C}(56)-\mathrm{H}(56 \mathrm{C})$ & 109.5 \\
$\mathrm{H}(56 \mathrm{~A})-\mathrm{C}(56)-\mathrm{H}(56 \mathrm{C})$ & 109.5 \\
$\mathrm{H}(56 \mathrm{~B})-\mathrm{C}(56)-\mathrm{H}(56 \mathrm{C})$ & 109.5 \\
$\mathrm{C}(56)-\mathrm{C}(55)-\mathrm{P}(3)$ & $113(3)$ \\
$\mathrm{C}(56)-\mathrm{C}(55)-\mathrm{H}(55 \mathrm{~A})$ & 109 \\
$\mathrm{P}(3)-\mathrm{C}(55)-\mathrm{H}(55 \mathrm{~A})$ & 109 \\
$\mathrm{C}(56)-\mathrm{C}(55)-\mathrm{H}(55 \mathrm{~B})$ & 109 \\
$\mathrm{P}(3)-\mathrm{C}(55)-\mathrm{H}(55 \mathrm{~B})$ & 109 \\
$\mathrm{H}(55 \mathrm{~A})-\mathrm{C}(55)-\mathrm{H}(55 \mathrm{~B})$ & 107.8 \\
$\mathrm{C}(58)-\mathrm{C}(57)-\mathrm{P}(3)$ & $113(3)$ \\
$\mathrm{C}(58)-\mathrm{C}(57)-\mathrm{H}(57 \mathrm{~A})$ & 109.1 \\
$\mathrm{P}(3)-\mathrm{C}(57)-\mathrm{H}(57 \mathrm{~A})$ & 109.1 \\
$\mathrm{C}(58)-\mathrm{C}(57)-\mathrm{H}(57 \mathrm{~B})$ & 109.1 \\
$\mathrm{P}(3)-\mathrm{C}(57)-\mathrm{H}(57 \mathrm{~B})$ & 109.1 \\
$\mathrm{H}(57 \mathrm{~A})-\mathrm{C}(57)-\mathrm{H}(57 \mathrm{~B})$ & 107.8 \\
$\mathrm{C}(57)-\mathrm{C}(58)-\mathrm{H}(58 \mathrm{~A})$ & 109.5 \\
$\mathrm{C}(57)-\mathrm{C}(58)-\mathrm{H}(58 \mathrm{~B})$ & 109.5 \\
$\mathrm{H}(58 \mathrm{~A})-\mathrm{C}(58)-\mathrm{H}(58 \mathrm{~B})$ & 109.5 \\
$\mathrm{C}(57)-\mathrm{C}(58)-\mathrm{H}(58 \mathrm{C})$ & 109.5 \\
$\mathrm{H}(58 \mathrm{~A})-\mathrm{C}(58)-\mathrm{H}(58 \mathrm{C})$ & 109.5 \\
$\mathrm{H}(58 \mathrm{~B})-\mathrm{C}(58)-\mathrm{H}(58 \mathrm{C})$ & 109.5 \\
\hline
\end{tabular}

Symmetry transformations used to generate equivalent atoms:

$\# 1-x+2,-y,-z+1$ 
Table 5. Atomic coordinates ( $\left.\mathrm{x} 10^{4}\right)$ and equivalent isotropic displacement parameters $\left(\AA^{2} \times 10^{3}\right)$ for BT3. U(eq) is defined as one third of the trace of the orthogonalized $\mathrm{U}^{\mathrm{ij}}$ tensor.

\begin{tabular}{lrrrr}
\hline & $\mathrm{x}$ & $\mathrm{y}$ & $\mathrm{z}$ & $\mathrm{U}(\mathrm{eq})$ \\
\hline $\mathrm{Pt}(1)$ & $6023(1)$ & $5750(2)$ & $4546(1)$ & $92(2)$ \\
$\mathrm{O}(1)$ & $4438(16)$ & $2350(40)$ & $2539(18)$ & $90(14)$ \\
$\mathrm{O}(2)$ & $3772(14)$ & $2770(30)$ & $2624(17)$ & $108(12)$ \\
$\mathrm{C}(23)$ & $4860(20)$ & $4450(30)$ & $3644(15)$ & $87(13)$ \\
$\mathrm{C}(24)$ & $5107(14)$ & $4030(40)$ & $3390(20)$ & $89(14)$ \\
$\mathrm{C}(25)$ & $4890(20)$ & $3490(30)$ & $3055(18)$ & $90(14)$ \\
$\mathrm{C}(26)$ & $4430(20)$ & $3370(30)$ & $2970(16)$ & $87(13)$ \\
$\mathrm{C}(27)$ & $4180(15)$ & $3790(40)$ & $3220(20)$ & $92(14)$ \\
$\mathrm{C}(28)$ & $4400(20)$ & $4330(30)$ & $3559(18)$ & $88(14)$ \\
$\mathrm{P}(1)$ & $6486(9)$ & $6353(17)$ & $5153(10)$ & $103(7)$ \\
$\mathrm{Pt}(2)$ & $3342(2)$ & $1949(4)$ & $2203(2)$ & $141(3)$ \\
$\mathrm{P}(2)$ & $6004(9)$ & $6885(14)$ & $4089(9)$ & $91(7)$ \\
$\mathrm{Zn}(03)$ & 5000 & 0 & 5000 & $82(5)$ \\
$\mathrm{O}(3)$ & $5615(18)$ & $5070(30)$ & $4027(17)$ & $81(9)$ \\
$\mathrm{C}(2)$ & $5586(12)$ & $4390(30)$ & $5084(15)$ & $67(12)$ \\
$\mathrm{N}(5)$ & $5949(12)$ & $4570(20)$ & $4908(13)$ & $47(8)$ \\
$\mathrm{C}(3)$ & $6177(11)$ & $3890(30)$ & $4767(13)$ & $34(8)$ \\
$\mathrm{C}(4)$ & $6043(14)$ & $3040(20)$ & $4803(15)$ & $55(12)$ \\
$\mathrm{C}(5)$ & $5681(15)$ & $2860(20)$ & $4979(16)$ & $67(11)$ \\
$\mathrm{C}(6)$ & $5453(13)$ & $3540(30)$ & $5120(16)$ & $72(13)$ \\
$\mathrm{O}(4)$ & $4957(18)$ & $5350(40)$ & $4230(20)$ & $100(15)$ \\
$\mathrm{P}(3)$ & $3184(10)$ & $2970(20)$ & $1691(9)$ & $124(7)$ \\
$\mathrm{P}(4)$ & $2867(11)$ & $990(30)$ & $1891(13)$ & $171(13)$ \\
$\mathrm{C}(7)$ & $5530(20)$ & $2030(50)$ & $5030(30)$ & $66(12)$ \\
$\mathrm{C}(8)$ & $5628(18)$ & $1590(30)$ & $5365(14)$ & $69(12)$ \\
$\mathrm{C}(9)$ & $5949(18)$ & $1840(30)$ & $5750(17)$ & $71(15)$ \\
$\mathrm{C}(10)$ & $5968(17)$ & $1170(40)$ & $6062(13)$ & $74(15)$ \\
$\mathrm{C}(11)$ & $5659(18)$ & $510(30)$ & $5870(17)$ & $74(14)$ \\
$\mathrm{N}(3)$ & $5449(15)$ & $770(30)$ & $5439(15)$ & $65(11)$ \\
$\mathrm{C}(12)$ & $5208(18)$ & $1790(40)$ & $4631(16)$ & $80(13)$ \\
$\mathrm{C}(13)$ & $5100(20)$ & $2270(30)$ & $4240(20)$ & $84(15)$ \\
$\mathrm{C}(14)$ & $4760(20)$ & $1820(40)$ & $3935(15)$ & $81(15)$ \\
$\mathrm{C}(15)$ & $4655(17)$ & $1060(30)$ & $4139(19)$ & $79(13)$ \\
$\mathrm{N}(2)$ & $4931(18)$ & $1040(30)$ & $4569(17)$ & $75(7)$ \\
$\mathrm{C}(16)$ & $4430(30)$ & $360(60)$ & $3980(30)$ & $77(9)$ \\
$\mathrm{C}(17)$ & $4180(30)$ & $450(60)$ & $3560(30)$ & $75(14)$
\end{tabular}




\begin{tabular}{|c|c|c|c|c|}
\hline$C(18)$ & $4180(30)$ & $300(60)$ & $3180(30)$ & $85(14)$ \\
\hline C(19) & $3990(30)$ & $580(70)$ & $2800(30)$ & $96(15)$ \\
\hline $\mathrm{N}(4)$ & $3640(30)$ & $1130(60)$ & $2740(30)$ & $118(15)$ \\
\hline$C(21)$ & $3470(30)$ & $1290(60)$ & $3080(30)$ & $98(16)$ \\
\hline $\mathrm{C}(20)$ & $3760(30)$ & $940(50)$ & $3510(30)$ & $76(14)$ \\
\hline$C(1 \mathrm{~A})$ & $5710(30)$ & $7640(50)$ & $4100(30)$ & $105(14)$ \\
\hline$C(2 A)$ & $5190(30)$ & $7550(60)$ & $3970(40)$ & $140(30)$ \\
\hline $\mathrm{C}(38)$ & $5910(30)$ & $6620(50)$ & $3500(30)$ & $110(14)$ \\
\hline C(39) & $6150(30)$ & $5760(50)$ & $3430(30)$ & $120(20)$ \\
\hline$C(3 \mathrm{~A})$ & $6650(30)$ & $7160(50)$ & $4170(30)$ & $118(16)$ \\
\hline C(49) & $2940(30)$ & $-160(70)$ & $2110(40)$ & $170(20)$ \\
\hline$C(52)$ & $5090(30)$ & $5050(40)$ & $3980(20)$ & $86(12)$ \\
\hline$C(53)$ & $4161(17)$ & $2750(50)$ & $2650(20)$ & $90(13)$ \\
\hline $\mathrm{C}(9 \mathrm{~A})$ & $3390(20)$ & $2420(50)$ & $1260(20)$ & $125(9)$ \\
\hline$C(12 \mathrm{~A})$ & $6440(30)$ & $5740(70)$ & $5680(20)$ & $128(17)$ \\
\hline$C(13 \mathrm{~A})$ & $3880(20)$ & $2320(70)$ & $1230(30)$ & $136(12)$ \\
\hline$C(14 A)$ & $6983(18)$ & $6420(70)$ & $4340(40)$ & $160(30)$ \\
\hline$C(15 A)$ & $5820(20)$ & $7440(60)$ & $5270(30)$ & $110(16)$ \\
\hline$C(16 A)$ & $6700(30)$ & $6050(60)$ & $6130(30)$ & $130(30)$ \\
\hline$C(17 \mathrm{~A})$ & $6320(20)$ & $7450(30)$ & $5270(30)$ & $96(13)$ \\
\hline$C(19 A)$ & $2570(40)$ & $-850(50)$ & $1930(40)$ & $200(40)$ \\
\hline$C(22 \mathrm{~A})$ & $1980(30)$ & $1010(100)$ & $2120(50)$ & $240(40)$ \\
\hline$C(23 \mathrm{~A})$ & $2360(30)$ & $730(90)$ & $1910(40)$ & $200(20)$ \\
\hline$C(25 \mathrm{~A})$ & $7054(17)$ & $6250(40)$ & $5080(30)$ & $119(15)$ \\
\hline$C(28 \mathrm{~A})$ & $7330(20)$ & $5410(60)$ & $5130(40)$ & $170(40)$ \\
\hline$C(29 A)$ & $2400(40)$ & $710(80)$ & $950(20)$ & $210(40)$ \\
\hline$C(31 \mathrm{~A})$ & $2800(30)$ & $570(70)$ & $1348(19)$ & 174(19) \\
\hline$C(32 \mathrm{~A})$ & $2890(30)$ & $3870(50)$ & $1360(30)$ & $136(17)$ \\
\hline$C(33 \mathrm{~A})$ & $3060(40)$ & $4330(70)$ & $1000(30)$ & $190(40)$ \\
\hline$C(34 \mathrm{~A})$ & $3530(20)$ & $3940(30)$ & $1720(30)$ & $136(16)$ \\
\hline$C(35 \mathrm{~A})$ & $3420(30)$ & $4870(30)$ & $1830(30)$ & $150(20)$ \\
\hline
\end{tabular}

Table 6. Bond lengths $[\AA]$ and angles $\left[^{\circ}\right]$ for BT3.

\begin{tabular}{lr}
\hline $\mathrm{Pt}(1)-\mathrm{O}(3)$ & $2.09(6)$ \\
$\operatorname{Pt}(1)-\mathrm{N}(5)$ & $2.19(3)$ \\
$\mathrm{Pt}(1)-\mathrm{P}(2)$ & $2.26(2)$ \\
$\mathrm{Pt}(1)-\mathrm{P}(1)$ & $2.29(3)$ \\
$\mathrm{O}(1)-\mathrm{C}(53)$ & $1.17(2)$ \\
$\mathrm{O}(2)-\mathrm{C}(53)$ & $1.18(2)$ \\
$\mathrm{O}(2)-\mathrm{Pt}(2)$ & $2.06(2)$ \\
$\mathrm{C}(23)-\mathrm{C}(24)$ & 1.39
\end{tabular}




\begin{tabular}{|c|c|}
\hline$C(23)-C(28)$ & 1.39 \\
\hline$C(23)-C(52)$ & $1.46(2)$ \\
\hline$C(24)-C(25)$ & 1.39 \\
\hline $\mathrm{C}(24)-\mathrm{H}(24)$ & 0.95 \\
\hline$C(25)-C(26)$ & 1.39 \\
\hline $\mathrm{C}(25)-\mathrm{H}(25)$ & 0.95 \\
\hline$C(26)-C(27)$ & 1.39 \\
\hline$C(26)-C(53)$ & $1.48(2)$ \\
\hline $\mathrm{C}(27)-\mathrm{C}(28)$ & 1.39 \\
\hline $\mathrm{C}(27)-\mathrm{H}(27)$ & 0.95 \\
\hline C(28)-H(28) & 0.95 \\
\hline$P(1)-C(25 A)$ & $1.82(2)$ \\
\hline $\mathrm{P}(1)-\mathrm{C}(17 \mathrm{~A})$ & $1.83(2)$ \\
\hline $\mathrm{P}(1)-\mathrm{C}(12 \mathrm{~A})$ & $1.96(7)$ \\
\hline $\operatorname{Pt}(2)-\mathrm{N}(4)$ & $2.13(9)$ \\
\hline $\mathrm{Pt}(2)-\mathrm{P}(4)$ & $2.13(3)$ \\
\hline $\operatorname{Pt}(2)-\mathrm{P}(3)$ & $2.23(3)$ \\
\hline$P(2)-C(1 A)$ & $1.48(8)$ \\
\hline $\mathrm{P}(2)-\mathrm{C}(38)$ & $1.87(9)$ \\
\hline$P(2)-C(3 A)$ & $1.96(9)$ \\
\hline $\mathrm{Zn}(03)-\mathrm{N}(3) \# 1$ & $2.07(4)$ \\
\hline $\mathrm{Zn}(03)-\mathrm{N}(3)$ & $2.07(4)$ \\
\hline $\mathrm{Zn}(03)-\mathrm{N}(2)$ & $2.08(4)$ \\
\hline $\mathrm{Zn}(03)-\mathrm{N}(2) \# 1$ & $2.08(4)$ \\
\hline $\mathrm{O}(3)-\mathrm{C}(52)$ & $1.58(10)$ \\
\hline $\mathrm{C}(2)-\mathrm{N}(5)$ & 1.39 \\
\hline$C(2)-C(6)$ & 1.39 \\
\hline $\mathrm{C}(2)-\mathrm{H}(2)$ & 0.95 \\
\hline $\mathrm{N}(5)-\mathrm{C}(3)$ & 1.39 \\
\hline $\mathrm{C}(3)-\mathrm{C}(4)$ & 1.39 \\
\hline $\mathrm{C}(3)-\mathrm{H}(3)$ & 0.95 \\
\hline$C(4)-C(5)$ & 1.39 \\
\hline $\mathrm{C}(4)-\mathrm{H}(4)$ & 0.95 \\
\hline$C(5)-C(7)$ & $1.37(8)$ \\
\hline$C(5)-C(6)$ & 1.39 \\
\hline $\mathrm{C}(6)-\mathrm{H}(6)$ & 0.95 \\
\hline $\mathrm{O}(4)-\mathrm{C}(52)$ & $1.07(8)$ \\
\hline $\mathrm{P}(3)-\mathrm{C}(34 \mathrm{~A})$ & $1.83(2)$ \\
\hline $\mathrm{P}(3)-\mathrm{C}(9 \mathrm{~A})$ & $1.85(2)$ \\
\hline$P(3)-C(32 A)$ & $1.83(7)$ \\
\hline$P(4)-C(23 A)$ & $1.63(9)$ \\
\hline $\mathrm{P}(4)-\mathrm{C}(31 \mathrm{~A})$ & $1.81(2)$ \\
\hline$P(4)-C(49)$ & $1.90(10)$ \\
\hline
\end{tabular}




\begin{tabular}{|c|c|}
\hline$C(7)-C(8)$ & $1.23(8)$ \\
\hline$C(7)-C(12)$ & $1.46(8)$ \\
\hline $\mathrm{C}(8)-\mathrm{N}(3)$ & 1.42 \\
\hline C(8)-C(9) & 1.42 \\
\hline $\mathrm{C}(9)-\mathrm{C}(10)$ & 1.42 \\
\hline $\mathrm{C}(9)-\mathrm{H}(9)$ & 0.95 \\
\hline $\mathrm{C}(10)-\mathrm{C}(11)$ & 1.42 \\
\hline $\mathrm{C}(10)-\mathrm{H}(10)$ & 0.95 \\
\hline $\mathrm{C}(11)-\mathrm{N}(3)$ & 1.42 \\
\hline$C(11)-C(16) \# 1$ & $1.46(9)$ \\
\hline C(12)-C(13) & 1.42 \\
\hline $\mathrm{C}(12)-\mathrm{N}(2)$ & 1.42 \\
\hline$C(13)-C(14)$ & 1.42 \\
\hline $\mathrm{C}(13)-\mathrm{H}(13)$ & 0.95 \\
\hline$C(14)-C(15)$ & 1.42 \\
\hline $\mathrm{C}(14)-\mathrm{H}(14)$ & 0.95 \\
\hline$C(15)-C(16)$ & $1.31(9)$ \\
\hline $\mathrm{C}(15)-\mathrm{N}(2)$ & 1.42 \\
\hline$C(16)-C(17)$ & $1.38(10)$ \\
\hline$C(17)-C(18)$ & $1.22(10)$ \\
\hline$C(17)-C(20)$ & $1.46(10)$ \\
\hline$C(18)-C(19)$ & $1.28(10)$ \\
\hline $\mathrm{C}(18)-\mathrm{H}(18)$ & 0.95 \\
\hline C(19)-N(4) & $1.35(11)$ \\
\hline C(19)-H(19) & 0.95 \\
\hline $\mathrm{N}(4)-\mathrm{C}(21)$ & $1.33(11)$ \\
\hline$C(21)-C(20)$ & $1.54(10)$ \\
\hline $\mathrm{C}(21)-\mathrm{H}(21)$ & 0.95 \\
\hline C(20)-H(20) & 0.95 \\
\hline$C(1 \mathrm{~A})-\mathrm{C}(2 \mathrm{~A})$ & $1.54(2)$ \\
\hline $\mathrm{C}(1 \mathrm{~A})-\mathrm{H}(1 \mathrm{~A} 1)$ & 0.99 \\
\hline $\mathrm{C}(1 \mathrm{~A})-\mathrm{H}(1 \mathrm{~A} 2)$ & 0.99 \\
\hline $\mathrm{C}(2 \mathrm{~A})-\mathrm{H}(2 \mathrm{~A} 1)$ & 0.989 \\
\hline $\mathrm{C}(2 \mathrm{~A})-\mathrm{H}(2 \mathrm{~A} 2)$ & 0.9894 \\
\hline $\mathrm{C}(2 \mathrm{~A})-\mathrm{H}(2 \mathrm{~A} 3)$ & 0.9893 \\
\hline C(38)-C(39) & $1.54(2)$ \\
\hline $\mathrm{C}(38)-\mathrm{H}(38 \mathrm{~A})$ & 0.99 \\
\hline $\mathrm{C}(38)-\mathrm{H}(38 \mathrm{~B})$ & 0.99 \\
\hline$C(39)-C(35 A) \# 2$ & $2.20(11)$ \\
\hline C(39)-H(39A) & 1.0085 \\
\hline C(39)-H(39B) & 1.0091 \\
\hline C(39)-H(39C) & 1.0095 \\
\hline$C(3 \mathrm{~A})-\mathrm{C}(14 \mathrm{~A})$ & $1.54(2)$ \\
\hline
\end{tabular}




\begin{tabular}{|c|c|}
\hline $\mathrm{C}(3 \mathrm{~A})-\mathrm{H}(3 \mathrm{~A} 1)$ & 0.99 \\
\hline $\mathrm{C}(3 \mathrm{~A})-\mathrm{H}(3 \mathrm{~A} 2)$ & 0.99 \\
\hline C(49)-C(19A) & $1.54(2)$ \\
\hline $\mathrm{C}(49)-\mathrm{H}(49 \mathrm{~A})$ & 0.99 \\
\hline C(49)-H(49B) & 0.99 \\
\hline$C(9 A)-C(13 A)$ & $1.55(2)$ \\
\hline $\mathrm{C}(9 \mathrm{~A})-\mathrm{H}(9 \mathrm{~A} 1)$ & 0.99 \\
\hline C(9A)-H(9A2) & 0.99 \\
\hline$C(12 A)-C(16 A)$ & $1.54(2)$ \\
\hline $\mathrm{C}(12 \mathrm{~A})-\mathrm{H}(12 \mathrm{~A})$ & 0.99 \\
\hline $\mathrm{C}(12 \mathrm{~A})-\mathrm{H}(12 \mathrm{~B})$ & 0.99 \\
\hline $\mathrm{C}(13 \mathrm{~A})-\mathrm{H}(13 \mathrm{~A})$ & 0.999 \\
\hline $\mathrm{C}(13 \mathrm{~A})-\mathrm{H}(13 \mathrm{~B})$ & 0.9967 \\
\hline $\mathrm{C}(13 \mathrm{~A})-\mathrm{H}(13 \mathrm{C})$ & 0.996 \\
\hline $\mathrm{C}(14 \mathrm{~A})-\mathrm{H}(14 \mathrm{~A})$ & 0.9801 \\
\hline $\mathrm{C}(14 \mathrm{~A})-\mathrm{H}(14 \mathrm{~B})$ & 0.9801 \\
\hline $\mathrm{C}(14 \mathrm{~A})-\mathrm{H}(14 \mathrm{C})$ & 0.9801 \\
\hline$C(15 A)-C(17 A)$ & $1.53(2)$ \\
\hline $\mathrm{C}(15 \mathrm{~A})-\mathrm{H}(15 \mathrm{~A})$ & 0.98 \\
\hline $\mathrm{C}(15 \mathrm{~A})-\mathrm{H}(15 \mathrm{~B})$ & 0.98 \\
\hline $\mathrm{C}(15 \mathrm{~A})-\mathrm{H}(15 \mathrm{C})$ & 0.98 \\
\hline $\mathrm{C}(16 \mathrm{~A})-\mathrm{H}(16 \mathrm{~A})$ & 0.9802 \\
\hline $\mathrm{C}(16 \mathrm{~A})-\mathrm{H}(16 \mathrm{~B})$ & 0.9802 \\
\hline$C(16 A)-H(16 C)$ & 0.9802 \\
\hline $\mathrm{C}(17 \mathrm{~A})-\mathrm{H}(17 \mathrm{~A})$ & 0.99 \\
\hline $\mathrm{C}(17 \mathrm{~A})-\mathrm{H}(17 \mathrm{~B})$ & 0.99 \\
\hline $\mathrm{C}(19 \mathrm{~A})-\mathrm{H}(19 \mathrm{~A})$ & 1.0149 \\
\hline C(19A)-H(19B) & 1.0127 \\
\hline C(19A)-H(19C) & 1.0131 \\
\hline$C(22 A)-C(23 A)$ & $1.54(2)$ \\
\hline $\mathrm{C}(22 \mathrm{~A})-\mathrm{H}(22 \mathrm{~A})$ & 1.1408 \\
\hline $\mathrm{C}(22 \mathrm{~A})-\mathrm{H}(22 \mathrm{~B})$ & 1.1551 \\
\hline $\mathrm{C}(22 \mathrm{~A})-\mathrm{H}(22 \mathrm{C})$ & 1.1401 \\
\hline $\mathrm{C}(23 \mathrm{~A})-\mathrm{H}(23 \mathrm{~A})$ & 0.99 \\
\hline $\mathrm{C}(23 \mathrm{~A})-\mathrm{H}(23 \mathrm{~B})$ & 0.99 \\
\hline$C(25 \mathrm{~A})-\mathrm{C}(28 \mathrm{~A})$ & $1.53(2)$ \\
\hline $\mathrm{C}(25 \mathrm{~A})-\mathrm{H}(25 \mathrm{~A})$ & 0.99 \\
\hline $\mathrm{C}(25 \mathrm{~A})-\mathrm{H}(25 \mathrm{~B})$ & 0.99 \\
\hline $\mathrm{C}(28 \mathrm{~A})-\mathrm{H}(28 \mathrm{~A})$ & 1.0135 \\
\hline $\mathrm{C}(28 \mathrm{~A})-\mathrm{H}(28 \mathrm{~B})$ & 1.0125 \\
\hline $\mathrm{C}(28 \mathrm{~A})-\mathrm{H}(28 \mathrm{C})$ & 1.0124 \\
\hline $\mathrm{C}(29 \mathrm{~A})-\mathrm{C}(31 \mathrm{~A})$ & $1.54(2)$ \\
\hline $\mathrm{C}(29 \mathrm{~A})-\mathrm{H}(29 \mathrm{~A})$ & 1.0091 \\
\hline
\end{tabular}




\begin{tabular}{|c|c|}
\hline $\mathrm{C}(29 \mathrm{~A})-\mathrm{H}(29 \mathrm{~B})$ & 1.0093 \\
\hline $\mathrm{C}(29 \mathrm{~A})-\mathrm{H}(29 \mathrm{C})$ & 1.0078 \\
\hline $\mathrm{C}(31 \mathrm{~A})-\mathrm{H}(31 \mathrm{~A})$ & 0.99 \\
\hline $\mathrm{C}(31 \mathrm{~A})-\mathrm{H}(31 \mathrm{~B})$ & 0.99 \\
\hline$C(32 A)-C(33 A)$ & $1.55(2)$ \\
\hline $\mathrm{C}(32 \mathrm{~A})-\mathrm{H}(32 \mathrm{~A})$ & 0.99 \\
\hline $\mathrm{C}(32 \mathrm{~A})-\mathrm{H}(32 \mathrm{~B})$ & 0.99 \\
\hline $\mathrm{C}(33 \mathrm{~A})-\mathrm{H}(33 \mathrm{~A})$ & 1.0001 \\
\hline $\mathrm{C}(33 \mathrm{~A})-\mathrm{H}(33 \mathrm{~B})$ & 1.0009 \\
\hline $\mathrm{C}(33 \mathrm{~A})-\mathrm{H}(33 \mathrm{C})$ & 0.9989 \\
\hline$C(34 A)-C(35 A)$ & $1.53(2)$ \\
\hline $\mathrm{C}(34 \mathrm{~A})-\mathrm{H}(34 \mathrm{~A})$ & 0.99 \\
\hline $\mathrm{C}(34 \mathrm{~A})-\mathrm{H}(34 \mathrm{~B})$ & 0.99 \\
\hline $\mathrm{C}(35 \mathrm{~A})-\mathrm{H}(35 \mathrm{~A})$ & 0.9801 \\
\hline $\mathrm{C}(35 \mathrm{~A})-\mathrm{H}(35 \mathrm{~B})$ & 0.9801 \\
\hline $\mathrm{C}(35 \mathrm{~A})-\mathrm{H}(35 \mathrm{C})$ & 0.9801 \\
\hline $\mathrm{O}(3)-\mathrm{Pt}(1)-\mathrm{N}(5)$ & $83.3(16)$ \\
\hline $\mathrm{O}(3)-\mathrm{Pt}(1)-\mathrm{P}(2)$ & $87.9(15)$ \\
\hline $\mathrm{N}(5)-\operatorname{Pt}(1)-\mathrm{P}(2)$ & $170.8(13)$ \\
\hline $\mathrm{O}(3)-\mathrm{Pt}(1)-\mathrm{P}(1)$ & $173.8(15)$ \\
\hline $\mathrm{N}(5)-\operatorname{Pt}(1)-\mathrm{P}(1)$ & $90.8(12)$ \\
\hline $\mathrm{P}(2)-\operatorname{Pt}(1)-\mathrm{P}(1)$ & $98.1(10)$ \\
\hline $\mathrm{C}(53)-\mathrm{O}(2)-\mathrm{Pt}(2)$ & $120(3)$ \\
\hline$C(24)-C(23)-C(28)$ & 120 \\
\hline$C(24)-C(23)-C(52)$ & $120(6)$ \\
\hline$C(28)-C(23)-C(52)$ & $120(6)$ \\
\hline $\mathrm{C}(25)-\mathrm{C}(24)-\mathrm{C}(23)$ & 120 \\
\hline $\mathrm{C}(25)-\mathrm{C}(24)-\mathrm{H}(24)$ & 120 \\
\hline $\mathrm{C}(23)-\mathrm{C}(24)-\mathrm{H}(24)$ & 120 \\
\hline$C(24)-C(25)-C(26)$ & 120 \\
\hline $\mathrm{C}(24)-\mathrm{C}(25)-\mathrm{H}(25)$ & 120 \\
\hline $\mathrm{C}(26)-\mathrm{C}(25)-\mathrm{H}(25)$ & 120 \\
\hline$C(25)-C(26)-C(27)$ & 120 \\
\hline$C(25)-C(26)-C(53)$ & $125(5)$ \\
\hline$C(27)-C(26)-C(53)$ & $115(5)$ \\
\hline $\mathrm{C}(28)-\mathrm{C}(27)-\mathrm{C}(26)$ & 120 \\
\hline $\mathrm{C}(28)-\mathrm{C}(27)-\mathrm{H}(27)$ & 120 \\
\hline $\mathrm{C}(26)-\mathrm{C}(27)-\mathrm{H}(27)$ & 120 \\
\hline $\mathrm{C}(27)-\mathrm{C}(28)-\mathrm{C}(23)$ & 120 \\
\hline $\mathrm{C}(27)-\mathrm{C}(28)-\mathrm{H}(28)$ & 120 \\
\hline $\mathrm{C}(23)-\mathrm{C}(28)-\mathrm{H}(28)$ & 120 \\
\hline$C(25 A)-P(1)-C(17 A)$ & $116(3)$ \\
\hline
\end{tabular}




\begin{tabular}{|c|c|}
\hline$C(25 A)-P(1)-C(12 A)$ & $110(3)$ \\
\hline$C(17 A)-P(1)-C(12 A)$ & $101(4)$ \\
\hline $\mathrm{C}(25 \mathrm{~A})-\mathrm{P}(1)-\mathrm{Pt}(1)$ & $106(4)$ \\
\hline $\mathrm{C}(17 \mathrm{~A})-\mathrm{P}(1)-\mathrm{Pt}(1)$ & $113(3)$ \\
\hline $\mathrm{C}(12 \mathrm{~A})-\mathrm{P}(1)-\mathrm{Pt}(1)$ & $111(3)$ \\
\hline $\mathrm{O}(2)-\mathrm{Pt}(2)-\mathrm{N}(4)$ & $76(3)$ \\
\hline $\mathrm{O}(2)-\mathrm{Pt}(2)-\mathrm{P}(4)$ & $168(2)$ \\
\hline $\mathrm{N}(4)-\operatorname{Pt}(2)-\mathrm{P}(4)$ & $94(2)$ \\
\hline $\mathrm{O}(2)-\mathrm{Pt}(2)-\mathrm{P}(3)$ & $91.9(19)$ \\
\hline $\mathrm{N}(4)-\operatorname{Pt}(2)-\mathrm{P}(3)$ & $167(2)$ \\
\hline $\mathrm{P}(4)-\mathrm{Pt}(2)-\mathrm{P}(3)$ & $98.3(13)$ \\
\hline $\mathrm{C}(1 \mathrm{~A})-\mathrm{P}(2)-\mathrm{C}(38)$ & $105(5)$ \\
\hline $\mathrm{C}(1 \mathrm{~A})-\mathrm{P}(2)-\mathrm{C}(3 \mathrm{~A})$ & $116(4)$ \\
\hline $\mathrm{C}(38)-\mathrm{P}(2)-\mathrm{C}(3 \mathrm{~A})$ & $94(5)$ \\
\hline C(1A)-P(2)-Pt(1) & $120(4)$ \\
\hline $\mathrm{C}(38)-\mathrm{P}(2)-\mathrm{Pt}(1)$ & $117(3)$ \\
\hline $\mathrm{C}(3 \mathrm{~A})-\mathrm{P}(2)-\mathrm{Pt}(1)$ & $103(2)$ \\
\hline $\mathrm{N}(3) \# 1-\mathrm{Zn}(03)-\mathrm{N}(3)$ & $180(5)$ \\
\hline $\mathrm{N}(3) \# 1-\mathrm{Zn}(03)-\mathrm{N}(2)$ & $93(5)$ \\
\hline $\mathrm{N}(3)-\mathrm{Zn}(03)-\mathrm{N}(2)$ & $87(2)$ \\
\hline $\mathrm{N}(3) \# 1-\mathrm{Zn}(03)-\mathrm{N}(2) \# 1$ & $87(7)$ \\
\hline $\mathrm{N}(3)-\mathrm{Zn}(03)-\mathrm{N}(2) \# 1$ & $93(6)$ \\
\hline $\mathrm{N}(2)-\mathrm{Zn}(03)-\mathrm{N}(2) \# 1$ & $180(6)$ \\
\hline $\mathrm{C}(52)-\mathrm{O}(3)-\mathrm{Pt}(1)$ & $118(3)$ \\
\hline $\mathrm{N}(5)-\mathrm{C}(2)-\mathrm{C}(6)$ & 120 \\
\hline $\mathrm{N}(5)-\mathrm{C}(2)-\mathrm{H}(2)$ & 120 \\
\hline $\mathrm{C}(6)-\mathrm{C}(2)-\mathrm{H}(2)$ & 120 \\
\hline $\mathrm{C}(2)-\mathrm{N}(5)-\mathrm{C}(3)$ & 120 \\
\hline $\mathrm{C}(2)-\mathrm{N}(5)-\mathrm{Pt}(1)$ & $125(2)$ \\
\hline $\mathrm{C}(3)-\mathrm{N}(5)-\mathrm{Pt}(1)$ & $109(2)$ \\
\hline $\mathrm{N}(5)-\mathrm{C}(3)-\mathrm{C}(4)$ & 120 \\
\hline $\mathrm{N}(5)-\mathrm{C}(3)-\mathrm{H}(3)$ & 120 \\
\hline $\mathrm{C}(4)-\mathrm{C}(3)-\mathrm{H}(3)$ & 120 \\
\hline$C(5)-C(4)-C(3)$ & 120 \\
\hline $\mathrm{C}(5)-\mathrm{C}(4)-\mathrm{H}(4)$ & 120 \\
\hline $\mathrm{C}(3)-\mathrm{C}(4)-\mathrm{H}(4)$ & 120 \\
\hline$C(7)-C(5)-C(4)$ & $123(5)$ \\
\hline$C(7)-C(5)-C(6)$ & $117(5)$ \\
\hline$C(4)-C(5)-C(6)$ & 120 \\
\hline$C(5)-C(6)-C(2)$ & 120 \\
\hline$C(5)-C(6)-H(6)$ & 120 \\
\hline $\mathrm{C}(2)-\mathrm{C}(6)-\mathrm{H}(6)$ & 120 \\
\hline $\mathrm{C}(34 \mathrm{~A})-\mathrm{P}(3)-\mathrm{C}(9 \mathrm{~A})$ & $96(2)$ \\
\hline
\end{tabular}




\begin{tabular}{|c|c|}
\hline $\mathrm{C}(34 \mathrm{~A})-\mathrm{P}(3)-\mathrm{C}(32 \mathrm{~A})$ & $67(4)$ \\
\hline $\mathrm{C}(9 \mathrm{~A})-\mathrm{P}(3)-\mathrm{C}(32 \mathrm{~A})$ & $97(3)$ \\
\hline $\mathrm{C}(34 \mathrm{~A})-\mathrm{P}(3)-\mathrm{Pt}(2)$ & $121(3)$ \\
\hline C(9A)-P(3)-Pt(2) & $100(3)$ \\
\hline $\mathrm{C}(32 \mathrm{~A})-\mathrm{P}(3)-\mathrm{Pt}(2)$ & $160(3)$ \\
\hline $\mathrm{C}(23 \mathrm{~A})-\mathrm{P}(4)-\mathrm{C}(31 \mathrm{~A})$ & $94(5)$ \\
\hline $\mathrm{C}(23 \mathrm{~A})-\mathrm{P}(4)-\mathrm{C}(49)$ & $77(6)$ \\
\hline $\mathrm{C}(31 \mathrm{~A})-\mathrm{P}(4)-\mathrm{C}(49)$ & $89(6)$ \\
\hline $\mathrm{C}(23 \mathrm{~A})-\mathrm{P}(4)-\mathrm{Pt}(2)$ & $135(4)$ \\
\hline $\mathrm{C}(31 \mathrm{~A})-\mathrm{P}(4)-\mathrm{Pt}(2)$ & $126(4)$ \\
\hline C(49)-P(4)-Pt(2) & $118(3)$ \\
\hline C(8)-C(7)-C(5) & $127(7)$ \\
\hline$C(8)-C(7)-C(12)$ & $125(7)$ \\
\hline$C(5)-C(7)-C(12)$ & $108(6)$ \\
\hline $\mathrm{C}(7)-\mathrm{C}(8)-\mathrm{N}(3)$ & $128(6)$ \\
\hline $\mathrm{C}(7)-\mathrm{C}(8)-\mathrm{C}(9)$ & $124(6)$ \\
\hline $\mathrm{N}(3)-\mathrm{C}(8)-\mathrm{C}(9)$ & 108 \\
\hline $\mathrm{C}(8)-\mathrm{C}(9)-\mathrm{C}(10)$ & 108 \\
\hline $\mathrm{C}(8)-\mathrm{C}(9)-\mathrm{H}(9)$ & 126 \\
\hline C(10)-C(9)-H(9) & 126 \\
\hline$C(9)-C(10)-C(11)$ & 108 \\
\hline $\mathrm{C}(9)-\mathrm{C}(10)-\mathrm{H}(10)$ & 126 \\
\hline $\mathrm{C}(11)-\mathrm{C}(10)-\mathrm{H}(10)$ & 126 \\
\hline $\mathrm{N}(3)-\mathrm{C}(11)-\mathrm{C}(10)$ & 108 \\
\hline$N(3)-C(11)-C(16) \# 1$ & $119(5)$ \\
\hline $\mathrm{C}(10)-\mathrm{C}(11)-\mathrm{C}(16) \# 1$ & $132(5)$ \\
\hline $\mathrm{C}(8)-\mathrm{N}(3)-\mathrm{C}(11)$ & 108 \\
\hline $\mathrm{C}(8)-\mathrm{N}(3)-\mathrm{Zn}(03)$ & $128(3)$ \\
\hline $\mathrm{C}(11)-\mathrm{N}(3)-\mathrm{Zn}(03)$ & $124(3)$ \\
\hline $\mathrm{C}(13)-\mathrm{C}(12)-\mathrm{N}(2)$ & 108 \\
\hline$C(13)-C(12)-C(7)$ & $126(5)$ \\
\hline $\mathrm{N}(2)-\mathrm{C}(12)-\mathrm{C}(7)$ & $126(5)$ \\
\hline$C(12)-C(13)-C(14)$ & 108 \\
\hline $\mathrm{C}(12)-\mathrm{C}(13)-\mathrm{H}(13)$ & 126 \\
\hline $\mathrm{C}(14)-\mathrm{C}(13)-\mathrm{H}(13)$ & 126 \\
\hline$C(15)-C(14)-C(13)$ & 108 \\
\hline $\mathrm{C}(15)-\mathrm{C}(14)-\mathrm{H}(14)$ & 126 \\
\hline $\mathrm{C}(13)-\mathrm{C}(14)-\mathrm{H}(14)$ & 126 \\
\hline$C(16)-C(15)-C(14)$ & $132(6)$ \\
\hline $\mathrm{C}(16)-\mathrm{C}(15)-\mathrm{N}(2)$ & $119(6)$ \\
\hline $\mathrm{C}(14)-\mathrm{C}(15)-\mathrm{N}(2)$ & 108 \\
\hline $\mathrm{C}(15)-\mathrm{N}(2)-\mathrm{C}(12)$ & 108 \\
\hline $\mathrm{C}(15)-\mathrm{N}(2)-\mathrm{Zn}(03)$ & $126(4)$ \\
\hline
\end{tabular}




\begin{tabular}{|c|c|}
\hline $\mathrm{C}(12)-\mathrm{N}(2)-\mathrm{Zn}(03)$ & $125(3)$ \\
\hline$C(15)-C(16)-C(17)$ & $113(8)$ \\
\hline$C(15)-C(16)-C(11) \# 1$ & $139(8)$ \\
\hline$C(17)-C(16)-C(11) \# 1$ & $108(7)$ \\
\hline $\mathrm{C}(18)-\mathrm{C}(17)-\mathrm{C}(16)$ & $144(10)$ \\
\hline$C(18)-C(17)-C(20)$ & $102(9)$ \\
\hline$C(16)-C(17)-C(20)$ & $114(8)$ \\
\hline $\mathrm{C}(17)-\mathrm{C}(18)-\mathrm{C}(19)$ & $140(10)$ \\
\hline $\mathrm{C}(17)-\mathrm{C}(18)-\mathrm{H}(18)$ & 110.4 \\
\hline $\mathrm{C}(19)-\mathrm{C}(18)-\mathrm{H}(18)$ & 110.1 \\
\hline $\mathrm{C}(18)-\mathrm{C}(19)-\mathrm{N}(4)$ & $120(10)$ \\
\hline $\mathrm{C}(18)-\mathrm{C}(19)-\mathrm{H}(19)$ & 119.9 \\
\hline N(4)-C(19)-H(19) & 120 \\
\hline $\mathrm{C}(21)-\mathrm{N}(4)-\mathrm{C}(19)$ & $117(9)$ \\
\hline $\mathrm{C}(21)-\mathrm{N}(4)-\mathrm{Pt}(2)$ & $112(8)$ \\
\hline C(19)-N(4)-Pt(2) & $131(8)$ \\
\hline $\mathrm{N}(4)-\mathrm{C}(21)-\mathrm{C}(20)$ & $114(9)$ \\
\hline $\mathrm{N}(4)-\mathrm{C}(21)-\mathrm{H}(21)$ & 123.1 \\
\hline $\mathrm{C}(20)-\mathrm{C}(21)-\mathrm{H}(21)$ & 123.2 \\
\hline$C(17)-C(20)-C(21)$ & $125(9)$ \\
\hline $\mathrm{C}(17)-\mathrm{C}(20)-\mathrm{H}(20)$ & 117.6 \\
\hline $\mathrm{C}(21)-\mathrm{C}(20)-\mathrm{H}(20)$ & 117.4 \\
\hline$P(2)-C(1 A)-C(2 A)$ & $121(6)$ \\
\hline $\mathrm{P}(2)-\mathrm{C}(1 \mathrm{~A})-\mathrm{H}(1 \mathrm{~A} 1)$ & 107 \\
\hline $\mathrm{C}(2 \mathrm{~A})-\mathrm{C}(1 \mathrm{~A})-\mathrm{H}(1 \mathrm{~A} 1)$ & 106.9 \\
\hline $\mathrm{P}(2)-\mathrm{C}(1 \mathrm{~A})-\mathrm{H}(1 \mathrm{~A} 2)$ & 107 \\
\hline $\mathrm{C}(2 \mathrm{~A})-\mathrm{C}(1 \mathrm{~A})-\mathrm{H}(1 \mathrm{~A} 2)$ & 107 \\
\hline $\mathrm{H}(1 \mathrm{~A} 1)-\mathrm{C}(1 \mathrm{~A})-\mathrm{H}(1 \mathrm{~A} 2)$ & 106.8 \\
\hline $\mathrm{C}(1 \mathrm{~A})-\mathrm{C}(2 \mathrm{~A})-\mathrm{H}(2 \mathrm{~A} 1)$ & 110.1 \\
\hline $\mathrm{C}(1 \mathrm{~A})-\mathrm{C}(2 \mathrm{~A})-\mathrm{H}(2 \mathrm{~A} 2)$ & 110.6 \\
\hline $\mathrm{H}(2 \mathrm{~A} 1)-\mathrm{C}(2 \mathrm{~A})-\mathrm{H}(2 \mathrm{~A} 2)$ & 108.5 \\
\hline $\mathrm{C}(1 \mathrm{~A})-\mathrm{C}(2 \mathrm{~A})-\mathrm{H}(2 \mathrm{~A} 3)$ & 110.4 \\
\hline $\mathrm{H}(2 \mathrm{~A} 1)-\mathrm{C}(2 \mathrm{~A})-\mathrm{H}(2 \mathrm{~A} 3)$ & 108.6 \\
\hline $\mathrm{H}(2 \mathrm{~A} 2)-\mathrm{C}(2 \mathrm{~A})-\mathrm{H}(2 \mathrm{~A} 3)$ & 108.6 \\
\hline $\mathrm{C}(39)-\mathrm{C}(38)-\mathrm{P}(2)$ & $112(6)$ \\
\hline $\mathrm{C}(39)-\mathrm{C}(38)-\mathrm{H}(38 \mathrm{~A})$ & 109.1 \\
\hline $\mathrm{P}(2)-\mathrm{C}(38)-\mathrm{H}(38 \mathrm{~A})$ & 109.4 \\
\hline C(39)-C(38)-H(38B) & 109.3 \\
\hline $\mathrm{P}(2)-\mathrm{C}(38)-\mathrm{H}(38 \mathrm{~B})$ & 109.3 \\
\hline H(38A)-C(38)-H(38B) & 107.9 \\
\hline$C(38)-C(39)-C(35 A) \# 2$ & $159(7)$ \\
\hline $\mathrm{C}(38)-\mathrm{C}(39)-\mathrm{H}(39 \mathrm{~A})$ & 112.1 \\
\hline $\mathrm{C}(35 \mathrm{~A}) \# 2-\mathrm{C}(39)-\mathrm{H}(39 \mathrm{~A})$ & 61.4 \\
\hline
\end{tabular}




\begin{tabular}{|c|c|}
\hline $\mathrm{C}(38)-\mathrm{C}(39)-\mathrm{H}(39 \mathrm{~B})$ & 112.3 \\
\hline $\mathrm{C}(35 \mathrm{~A}) \# 2-\mathrm{C}(39)-\mathrm{H}(39 \mathrm{~B})$ & 56 \\
\hline H(39A)-C(39)-H(39B) & 106.6 \\
\hline $\mathrm{C}(38)-\mathrm{C}(39)-\mathrm{H}(39 \mathrm{C})$ & 112.5 \\
\hline $\mathrm{C}(35 \mathrm{~A}) \# 2-\mathrm{C}(39)-\mathrm{H}(39 \mathrm{C})$ & 88.7 \\
\hline $\mathrm{H}(39 \mathrm{~A})-\mathrm{C}(39)-\mathrm{H}(39 \mathrm{C})$ & 106.5 \\
\hline $\mathrm{H}(39 \mathrm{~B})-\mathrm{C}(39)-\mathrm{H}(39 \mathrm{C})$ & 106.5 \\
\hline $\mathrm{C}(14 \mathrm{~A})-\mathrm{C}(3 \mathrm{~A})-\mathrm{P}(2)$ & $117(5)$ \\
\hline $\mathrm{C}(14 \mathrm{~A})-\mathrm{C}(3 \mathrm{~A})-\mathrm{H}(3 \mathrm{~A} 1)$ & 108.3 \\
\hline $\mathrm{P}(2)-\mathrm{C}(3 \mathrm{~A})-\mathrm{H}(3 \mathrm{~A} 1)$ & 108.2 \\
\hline $\mathrm{C}(14 \mathrm{~A})-\mathrm{C}(3 \mathrm{~A})-\mathrm{H}(3 \mathrm{~A} 2)$ & 108.2 \\
\hline $\mathrm{P}(2)-\mathrm{C}(3 \mathrm{~A})-\mathrm{H}(3 \mathrm{~A} 2)$ & 107.9 \\
\hline $\mathrm{H}(3 \mathrm{~A} 1)-\mathrm{C}(3 \mathrm{~A})-\mathrm{H}(3 \mathrm{~A} 2)$ & 107.3 \\
\hline C(19A)-C(49)-P(4) & $120(6)$ \\
\hline $\mathrm{C}(19 \mathrm{~A})-\mathrm{C}(49)-\mathrm{H}(49 \mathrm{~A})$ & 107.5 \\
\hline $\mathrm{P}(4)-\mathrm{C}(49)-\mathrm{H}(49 \mathrm{~A})$ & 107.5 \\
\hline $\mathrm{C}(19 \mathrm{~A})-\mathrm{C}(49)-\mathrm{H}(49 \mathrm{~B})$ & 106.9 \\
\hline $\mathrm{P}(4)-\mathrm{C}(49)-\mathrm{H}(49 \mathrm{~B})$ & 107.3 \\
\hline $\mathrm{H}(49 \mathrm{~A})-\mathrm{C}(49)-\mathrm{H}(49 \mathrm{~B})$ & 106.9 \\
\hline $\mathrm{O}(4)-\mathrm{C}(52)-\mathrm{C}(23)$ & $128(9)$ \\
\hline $\mathrm{O}(4)-\mathrm{C}(52)-\mathrm{O}(3)$ & $119(6)$ \\
\hline $\mathrm{C}(23)-\mathrm{C}(52)-\mathrm{O}(3)$ & $111(7)$ \\
\hline $\mathrm{O}(1)-\mathrm{C}(53)-\mathrm{O}(2)$ & $142(5)$ \\
\hline $\mathrm{O}(1)-\mathrm{C}(53)-\mathrm{C}(26)$ & $104(5)$ \\
\hline $\mathrm{O}(2)-\mathrm{C}(53)-\mathrm{C}(26)$ & $114(4)$ \\
\hline$C(13 A)-C(9 A)-P(3)$ & $127(3)$ \\
\hline $\mathrm{C}(13 \mathrm{~A})-\mathrm{C}(9 \mathrm{~A})-\mathrm{H}(9 \mathrm{~A} 1)$ & 106.3 \\
\hline $\mathrm{P}(3)-\mathrm{C}(9 \mathrm{~A})-\mathrm{H}(9 \mathrm{~A} 1)$ & 105.7 \\
\hline $\mathrm{C}(13 \mathrm{~A})-\mathrm{C}(9 \mathrm{~A})-\mathrm{H}(9 \mathrm{~A} 2)$ & 104.7 \\
\hline $\mathrm{P}(3)-\mathrm{C}(9 \mathrm{~A})-\mathrm{H}(9 \mathrm{~A} 2)$ & 105.6 \\
\hline $\mathrm{H}(9 \mathrm{~A} 1)-\mathrm{C}(9 \mathrm{~A})-\mathrm{H}(9 \mathrm{~A} 2)$ & 106.1 \\
\hline C(16A)-C(12A)-P(1) & $121(6)$ \\
\hline $\mathrm{C}(16 \mathrm{~A})-\mathrm{C}(12 \mathrm{~A})-\mathrm{H}(12 \mathrm{~A})$ & 107.2 \\
\hline $\mathrm{P}(1)-\mathrm{C}(12 \mathrm{~A})-\mathrm{H}(12 \mathrm{~A})$ & 107.2 \\
\hline $\mathrm{C}(16 \mathrm{~A})-\mathrm{C}(12 \mathrm{~A})-\mathrm{H}(12 \mathrm{~B})$ & 106.9 \\
\hline $\mathrm{P}(1)-\mathrm{C}(12 \mathrm{~A})-\mathrm{H}(12 \mathrm{~B})$ & 107 \\
\hline $\mathrm{H}(12 \mathrm{~A})-\mathrm{C}(12 \mathrm{~A})-\mathrm{H}(12 \mathrm{~B})$ & 106.8 \\
\hline $\mathrm{C}(9 \mathrm{~A})-\mathrm{C}(13 \mathrm{~A})-\mathrm{H}(13 \mathrm{~A})$ & 112.2 \\
\hline $\mathrm{C}(9 \mathrm{~A})-\mathrm{C}(13 \mathrm{~A})-\mathrm{H}(13 \mathrm{~B})$ & 110.9 \\
\hline $\mathrm{H}(13 \mathrm{~A})-\mathrm{C}(13 \mathrm{~A})-\mathrm{H}(13 \mathrm{~B})$ & 107.9 \\
\hline C(9A)-C(13A)-H(13C) & 110.4 \\
\hline $\mathrm{H}(13 \mathrm{~A})-\mathrm{C}(13 \mathrm{~A})-\mathrm{H}(13 \mathrm{C})$ & 107.5 \\
\hline $\mathrm{H}(13 \mathrm{~B})-\mathrm{C}(13 \mathrm{~A})-\mathrm{H}(13 \mathrm{C})$ & 107.9 \\
\hline
\end{tabular}




\begin{tabular}{|c|c|}
\hline $\mathrm{C}(3 \mathrm{~A})-\mathrm{C}(14 \mathrm{~A})-\mathrm{H}(14 \mathrm{~A})$ & 109.6 \\
\hline $\mathrm{C}(3 \mathrm{~A})-\mathrm{C}(14 \mathrm{~A})-\mathrm{H}(14 \mathrm{~B})$ & 109.3 \\
\hline $\mathrm{H}(14 \mathrm{~A})-\mathrm{C}(14 \mathrm{~A})-\mathrm{H}(14 \mathrm{~B})$ & 109.5 \\
\hline $\mathrm{C}(3 \mathrm{~A})-\mathrm{C}(14 \mathrm{~A})-\mathrm{H}(14 \mathrm{C})$ & 109.6 \\
\hline $\mathrm{H}(14 \mathrm{~A})-\mathrm{C}(14 \mathrm{~A})-\mathrm{H}(14 \mathrm{C})$ & 109.5 \\
\hline $\mathrm{H}(14 \mathrm{~B})-\mathrm{C}(14 \mathrm{~A})-\mathrm{H}(14 \mathrm{C})$ & 109.5 \\
\hline $\mathrm{C}(17 \mathrm{~A})-\mathrm{C}(15 \mathrm{~A})-\mathrm{H}(15 \mathrm{~A})$ & 109.5 \\
\hline $\mathrm{C}(17 \mathrm{~A})-\mathrm{C}(15 \mathrm{~A})-\mathrm{H}(15 \mathrm{~B})$ & 109.5 \\
\hline $\mathrm{H}(15 \mathrm{~A})-\mathrm{C}(15 \mathrm{~A})-\mathrm{H}(15 \mathrm{~B})$ & 109.5 \\
\hline$C(17 A)-C(15 A)-H(15 C)$ & 109.4 \\
\hline $\mathrm{H}(15 \mathrm{~A})-\mathrm{C}(15 \mathrm{~A})-\mathrm{H}(15 \mathrm{C})$ & 109.5 \\
\hline $\mathrm{H}(15 \mathrm{~B})-\mathrm{C}(15 \mathrm{~A})-\mathrm{H}(15 \mathrm{C})$ & 109.5 \\
\hline $\mathrm{C}(12 \mathrm{~A})-\mathrm{C}(16 \mathrm{~A})-\mathrm{H}(16 \mathrm{~A})$ & 109.3 \\
\hline $\mathrm{C}(12 \mathrm{~A})-\mathrm{C}(16 \mathrm{~A})-\mathrm{H}(16 \mathrm{~B})$ & 109.6 \\
\hline $\mathrm{H}(16 \mathrm{~A})-\mathrm{C}(16 \mathrm{~A})-\mathrm{H}(16 \mathrm{~B})$ & 109.5 \\
\hline$C(12 A)-C(16 A)-H(16 C)$ & 109.5 \\
\hline $\mathrm{H}(16 \mathrm{~A})-\mathrm{C}(16 \mathrm{~A})-\mathrm{H}(16 \mathrm{C})$ & 109.4 \\
\hline $\mathrm{H}(16 \mathrm{~B})-\mathrm{C}(16 \mathrm{~A})-\mathrm{H}(16 \mathrm{C})$ & 109.5 \\
\hline $\mathrm{C}(15 \mathrm{~A})-\mathrm{C}(17 \mathrm{~A})-\mathrm{P}(1)$ & $109(5)$ \\
\hline$C(15 A)-C(17 A)-H(17 A)$ & 110 \\
\hline $\mathrm{P}(1)-\mathrm{C}(17 \mathrm{~A})-\mathrm{H}(17 \mathrm{~A})$ & 109.8 \\
\hline $\mathrm{C}(15 \mathrm{~A})-\mathrm{C}(17 \mathrm{~A})-\mathrm{H}(17 \mathrm{~B})$ & 109.9 \\
\hline $\mathrm{P}(1)-\mathrm{C}(17 \mathrm{~A})-\mathrm{H}(17 \mathrm{~B})$ & 110.1 \\
\hline $\mathrm{H}(17 \mathrm{~A})-\mathrm{C}(17 \mathrm{~A})-\mathrm{H}(17 \mathrm{~B})$ & 108.3 \\
\hline C(49)-C(19A)-H(19A) & 113.2 \\
\hline C(49)-C(19A)-H(19B) & 112.5 \\
\hline $\mathrm{H}(19 \mathrm{~A})-\mathrm{C}(19 \mathrm{~A})-\mathrm{H}(19 \mathrm{~B})$ & 106.1 \\
\hline C(49)-C(19A)-H(19C) & 112.6 \\
\hline $\mathrm{H}(19 \mathrm{~A})-\mathrm{C}(19 \mathrm{~A})-\mathrm{H}(19 \mathrm{C})$ & 105.9 \\
\hline $\mathrm{H}(19 \mathrm{~B})-\mathrm{C}(19 \mathrm{~A})-\mathrm{H}(19 \mathrm{C})$ & 106.1 \\
\hline $\mathrm{C}(23 \mathrm{~A})-\mathrm{C}(22 \mathrm{~A})-\mathrm{H}(22 \mathrm{~A})$ & 125.4 \\
\hline $\mathrm{C}(23 \mathrm{~A})-\mathrm{C}(22 \mathrm{~A})-\mathrm{H}(22 \mathrm{~B})$ & 127.3 \\
\hline $\mathrm{H}(22 \mathrm{~A})-\mathrm{C}(22 \mathrm{~A})-\mathrm{H}(22 \mathrm{~B})$ & 88.7 \\
\hline $\mathrm{C}(23 \mathrm{~A})-\mathrm{C}(22 \mathrm{~A})-\mathrm{H}(22 \mathrm{C})$ & 124.6 \\
\hline $\mathrm{H}(22 \mathrm{~A})-\mathrm{C}(22 \mathrm{~A})-\mathrm{H}(22 \mathrm{C})$ & 89.7 \\
\hline $\mathrm{H}(22 \mathrm{~B})-\mathrm{C}(22 \mathrm{~A})-\mathrm{H}(22 \mathrm{C})$ & 89.3 \\
\hline $\mathrm{C}(22 \mathrm{~A})-\mathrm{C}(23 \mathrm{~A})-\mathrm{P}(4)$ & $142(10)$ \\
\hline $\mathrm{C}(22 \mathrm{~A})-\mathrm{C}(23 \mathrm{~A})-\mathrm{H}(23 \mathrm{~A})$ & 101.6 \\
\hline $\mathrm{P}(4)-\mathrm{C}(23 \mathrm{~A})-\mathrm{H}(23 \mathrm{~A})$ & 102.1 \\
\hline $\mathrm{C}(22 \mathrm{~A})-\mathrm{C}(23 \mathrm{~A})-\mathrm{H}(23 \mathrm{~B})$ & 101.5 \\
\hline $\mathrm{P}(4)-\mathrm{C}(23 \mathrm{~A})-\mathrm{H}(23 \mathrm{~B})$ & 101.1 \\
\hline $\mathrm{H}(23 \mathrm{~A})-\mathrm{C}(23 \mathrm{~A})-\mathrm{H}(23 \mathrm{~B})$ & 104.6 \\
\hline $\mathrm{C}(28 \mathrm{~A})-\mathrm{C}(25 \mathrm{~A})-\mathrm{P}(1)$ & $126(3)$ \\
\hline
\end{tabular}




\begin{tabular}{|c|c|}
\hline $\mathrm{C}(28 \mathrm{~A})-\mathrm{C}(25 \mathrm{~A})-\mathrm{H}(25 \mathrm{~A})$ & 106.1 \\
\hline $\mathrm{P}(1)-\mathrm{C}(25 \mathrm{~A})-\mathrm{H}(25 \mathrm{~A})$ & 105.9 \\
\hline $\mathrm{C}(28 \mathrm{~A})-\mathrm{C}(25 \mathrm{~A})-\mathrm{H}(25 \mathrm{~B})$ & 105.8 \\
\hline $\mathrm{P}(1)-\mathrm{C}(25 \mathrm{~A})-\mathrm{H}(25 \mathrm{~B})$ & 105.9 \\
\hline $\mathrm{H}(25 \mathrm{~A})-\mathrm{C}(25 \mathrm{~A})-\mathrm{H}(25 \mathrm{~B})$ & 106.2 \\
\hline $\mathrm{C}(25 \mathrm{~A})-\mathrm{C}(28 \mathrm{~A})-\mathrm{H}(28 \mathrm{~A})$ & 112.9 \\
\hline $\mathrm{C}(25 \mathrm{~A})-\mathrm{C}(28 \mathrm{~A})-\mathrm{H}(28 \mathrm{~B})$ & 112.6 \\
\hline $\mathrm{H}(28 \mathrm{~A})-\mathrm{C}(28 \mathrm{~A})-\mathrm{H}(28 \mathrm{~B})$ & 106.1 \\
\hline $\mathrm{C}(25 \mathrm{~A})-\mathrm{C}(28 \mathrm{~A})-\mathrm{H}(28 \mathrm{C})$ & 112.6 \\
\hline $\mathrm{H}(28 \mathrm{~A})-\mathrm{C}(28 \mathrm{~A})-\mathrm{H}(28 \mathrm{C})$ & 106 \\
\hline $\mathrm{H}(28 \mathrm{~B})-\mathrm{C}(28 \mathrm{~A})-\mathrm{H}(28 \mathrm{C})$ & 106.1 \\
\hline$C(31 A)-C(29 A)-H(29 A)$ & 112.3 \\
\hline C(31A)-C(29A)-H(29B) & 112.4 \\
\hline $\mathrm{H}(29 \mathrm{~A})-\mathrm{C}(29 \mathrm{~A})-\mathrm{H}(29 \mathrm{~B})$ & 106.5 \\
\hline $\mathrm{C}(31 \mathrm{~A})-\mathrm{C}(29 \mathrm{~A})-\mathrm{H}(29 \mathrm{C})$ & 112.1 \\
\hline $\mathrm{H}(29 \mathrm{~A})-\mathrm{C}(29 \mathrm{~A})-\mathrm{H}(29 \mathrm{C})$ & 106.7 \\
\hline H(29B)-C(29A)-H(29C) & 106.5 \\
\hline $\mathrm{C}(29 \mathrm{~A})-\mathrm{C}(31 \mathrm{~A})-\mathrm{P}(4)$ & $127(4)$ \\
\hline $\mathrm{C}(29 \mathrm{~A})-\mathrm{C}(31 \mathrm{~A})-\mathrm{H}(31 \mathrm{~A})$ & 105.7 \\
\hline $\mathrm{P}(4)-\mathrm{C}(31 \mathrm{~A})-\mathrm{H}(31 \mathrm{~A})$ & 105.5 \\
\hline $\mathrm{C}(29 \mathrm{~A})-\mathrm{C}(31 \mathrm{~A})-\mathrm{H}(31 \mathrm{~B})$ & 105.5 \\
\hline $\mathrm{P}(4)-\mathrm{C}(31 \mathrm{~A})-\mathrm{H}(31 \mathrm{~B})$ & 105.6 \\
\hline $\mathrm{H}(31 \mathrm{~A})-\mathrm{C}(31 \mathrm{~A})-\mathrm{H}(31 \mathrm{~B})$ & 106.1 \\
\hline $\mathrm{C}(33 \mathrm{~A})-\mathrm{C}(32 \mathrm{~A})-\mathrm{P}(3)$ & $124(5)$ \\
\hline $\mathrm{C}(33 \mathrm{~A})-\mathrm{C}(32 \mathrm{~A})-\mathrm{H}(32 \mathrm{~A})$ & 106.8 \\
\hline $\mathrm{P}(3)-\mathrm{C}(32 \mathrm{~A})-\mathrm{H}(32 \mathrm{~A})$ & 106.2 \\
\hline $\mathrm{C}(33 \mathrm{~A})-\mathrm{C}(32 \mathrm{~A})-\mathrm{H}(32 \mathrm{~B})$ & 105.9 \\
\hline $\mathrm{P}(3)-\mathrm{C}(32 \mathrm{~A})-\mathrm{H}(32 \mathrm{~B})$ & 106.2 \\
\hline $\mathrm{H}(32 \mathrm{~A})-\mathrm{C}(32 \mathrm{~A})-\mathrm{H}(32 \mathrm{~B})$ & 106.4 \\
\hline $\mathrm{C}(32 \mathrm{~A})-\mathrm{C}(33 \mathrm{~A})-\mathrm{H}(33 \mathrm{~A})$ & 111.5 \\
\hline $\mathrm{C}(32 \mathrm{~A})-\mathrm{C}(33 \mathrm{~A})-\mathrm{H}(33 \mathrm{~B})$ & 111.9 \\
\hline $\mathrm{H}(33 \mathrm{~A})-\mathrm{C}(33 \mathrm{~A})-\mathrm{H}(33 \mathrm{~B})$ & 107.4 \\
\hline $\mathrm{C}(32 \mathrm{~A})-\mathrm{C}(33 \mathrm{~A})-\mathrm{H}(33 \mathrm{C})$ & 110.8 \\
\hline $\mathrm{H}(33 \mathrm{~A})-\mathrm{C}(33 \mathrm{~A})-\mathrm{H}(33 \mathrm{C})$ & 107.4 \\
\hline $\mathrm{H}(33 \mathrm{~B})-\mathrm{C}(33 \mathrm{~A})-\mathrm{H}(33 \mathrm{C})$ & 107.6 \\
\hline $\mathrm{C}(35 \mathrm{~A})-\mathrm{C}(34 \mathrm{~A})-\mathrm{P}(3)$ & $128(4)$ \\
\hline $\mathrm{C}(35 \mathrm{~A})-\mathrm{C}(34 \mathrm{~A})-\mathrm{H}(34 \mathrm{~A})$ & 104.9 \\
\hline $\mathrm{P}(3)-\mathrm{C}(34 \mathrm{~A})-\mathrm{H}(34 \mathrm{~A})$ & 105.3 \\
\hline $\mathrm{C}(35 \mathrm{~A})-\mathrm{C}(34 \mathrm{~A})-\mathrm{H}(34 \mathrm{~B})$ & 105.7 \\
\hline $\mathrm{P}(3)-\mathrm{C}(34 \mathrm{~A})-\mathrm{H}(34 \mathrm{~B})$ & 105.4 \\
\hline $\mathrm{H}(34 \mathrm{~A})-\mathrm{C}(34 \mathrm{~A})-\mathrm{H}(34 \mathrm{~B})$ & 106 \\
\hline $\mathrm{C}(34 \mathrm{~A})-\mathrm{C}(35 \mathrm{~A})-\mathrm{C}(39) \# 2$ & $108(6)$ \\
\hline $\mathrm{C}(34 \mathrm{~A})-\mathrm{C}(35 \mathrm{~A})-\mathrm{H}(35 \mathrm{~A})$ & 109.3 \\
\hline
\end{tabular}




\begin{tabular}{lr}
$\mathrm{C}(39) \# 2-\mathrm{C}(35 \mathrm{~A})-\mathrm{H}(35 \mathrm{~A})$ & 133.6 \\
$\mathrm{C}(34 \mathrm{~A})-\mathrm{C}(35 \mathrm{~A})-\mathrm{H}(35 \mathrm{~B})$ & 109.1 \\
$\mathrm{C}(39) \# 2-\mathrm{C}(35 \mathrm{~A})-\mathrm{H}(35 \mathrm{~B})$ & 30.7 \\
$\mathrm{H}(35 \mathrm{~A})-\mathrm{C}(35 \mathrm{~A})-\mathrm{H}(35 \mathrm{~B})$ & 109.5 \\
$\mathrm{C}(34 \mathrm{~A})-\mathrm{C}(35 \mathrm{~A})-\mathrm{H}(35 \mathrm{C})$ & 110 \\
$\mathrm{C}(39) \# 2-\mathrm{C}(35 \mathrm{~A})-\mathrm{H}(35 \mathrm{C})$ & 81.8 \\
$\mathrm{H}(35 \mathrm{~A})-\mathrm{C}(35 \mathrm{~A})-\mathrm{H}(35 \mathrm{C})$ & 109.5 \\
$\mathrm{H}(35 \mathrm{~B})-\mathrm{C}(35 \mathrm{~A})-\mathrm{H}(35 \mathrm{C})$ & 109.5 \\
\hline
\end{tabular}

Symmetry transformations used to generate equivalent atoms:

$\# 1-\mathrm{x}+1,-\mathrm{y},-\mathrm{z}+1 \quad \# 2-\mathrm{x}+1, \mathrm{y},-\mathrm{z}+1 / 2$

Table 7. Atomic coordinates $\left(\mathrm{x} 10^{4}\right)$ and equivalent isotropic displacement parameters $\left(\AA^{2} \times 10^{3}\right)$ for BT4. U(eq) is defined as one third of the trace of the orthogonalized $\mathrm{U}^{\mathrm{ij}}$ tensor.

\begin{tabular}{|c|c|c|c|c|}
\hline & $\mathrm{x}$ & $\mathrm{y}$ & $\mathrm{z}$ & $\mathrm{U}(\mathrm{eq})$ \\
\hline $\operatorname{Pt}(1)$ & $11311(1)$ & $5735(1)$ & 5811(1) & $95(1)$ \\
\hline $\operatorname{Pt}(2)$ & $7728(1)$ & $7226(1)$ & $636(1)$ & $127(1)$ \\
\hline $\operatorname{Pt}(3)$ & $2824(1)$ & 6741(1) & $3123(1)$ & $203(1)$ \\
\hline $\operatorname{Pt}(4)$ & $6519(1)$ & $5208(1)$ & $8238(1)$ & $172(1)$ \\
\hline $\mathrm{Zn}(1)$ & $7006(1)$ & $6042(1)$ & $4265(2)$ & $83(1)$ \\
\hline $\mathrm{P}(4)$ & $6875(7)$ & $7432(6)$ & $-16(7)$ & $196(6)$ \\
\hline $\mathrm{P}(5)$ & $2678(7)$ & $7130(8)$ & $2258(11)$ & $270(12)$ \\
\hline $\mathrm{P}(6)$ & 1911(7) & $6815(10)$ & $3164(12)$ & $349(17)$ \\
\hline $\mathrm{P}(7)$ & 6053(8) & $5041(7)$ & $8926(8)$ & $220(8)$ \\
\hline $\mathrm{P}(8)$ & $7423(9)$ & $5218(10)$ & $8884(10)$ & $311(15)$ \\
\hline $\mathrm{O}(1)$ & $11074(9)$ & $5931(6)$ & $4915(11)$ & $96(5)$ \\
\hline $\mathrm{O}(2)$ & $10664(9)$ & $5368(7)$ & $4489(10)$ & $98(6)$ \\
\hline $\mathrm{O}(3)$ & $8509(10)$ & $7020(8)$ & $1324(13)$ & $123(7)$ \\
\hline $\mathrm{O}(4)$ & $8572(12)$ & 7579(9) & 1931(14) & $143(9)$ \\
\hline $\mathrm{O}(5)$ & $3110(11)$ & $6473(12)$ & $4045(15)$ & $164(10)$ \\
\hline $\mathrm{O}(6)$ & $3109(16)$ & $5833(13)$ & $3583(19)$ & 197(13) \\
\hline $\mathrm{O}(8)$ & $5735(12)$ & $4709(9)$ & $7128(15)$ & $148(9)$ \\
\hline $\mathrm{O}(9 \mathrm{~W})$ & $6813(10)$ & $5532(6)$ & $3685(14)$ & $134(8)$ \\
\hline $\mathrm{N}(5)$ & $10369(10)$ & $5770(5)$ & $5630(12)$ & $79(6)$ \\
\hline C(29) & $7265(11)$ & $6464(5)$ & $1929(12)$ & $118(9)$ \\
\hline$C(26)$ & $7189(10)$ & $6720(7)$ & 2383(9) & $101(7)$ \\
\hline $\mathrm{C}(27)$ & $7199(10)$ & $7129(6)$ & $2297(10)$ & $109(8)$ \\
\hline $\mathrm{C}(28)$ & $7284(10)$ & $7282(5)$ & $1757(12)$ & $118(9)$ \\
\hline $\mathrm{N}(6)$ & $7360(9)$ & $7025(7)$ & 1304(9) & $112(7)$ \\
\hline $\mathrm{C}(30)$ & $7350(10)$ & $6616(7)$ & $1390(10)$ & $118(9)$ \\
\hline $\mathrm{P}(1)$ & $11480(4)$ & $5553(3)$ & $6803(6)$ & $118(3)$ \\
\hline
\end{tabular}




\begin{tabular}{|c|c|c|c|c|}
\hline $\mathrm{N}(8)$ & $6769(10)$ & $5365(8)$ & 7456(9) & $131(8)$ \\
\hline$C(36)$ & $6914(10)$ & $5087(6)$ & $7073(12)$ & $133(10)$ \\
\hline$C(37)$ & $6976(10)$ & $5206(7)$ & $6504(11)$ & $120(9)$ \\
\hline$C(38)$ & $6892(10)$ & $5602(8)$ & $6318(10)$ & $113(8)$ \\
\hline$C(39)$ & $6747(10)$ & $5880(6)$ & $6702(13)$ & $124(9)$ \\
\hline $\mathrm{C}(40)$ & $6685(10)$ & $5761(7)$ & $7271(11)$ & $134(9)$ \\
\hline$C(34)$ & $4679(16)$ & $6780(13)$ & $3820(20)$ & $151(12)$ \\
\hline$C(31)$ & $4895(14)$ & $6427(11)$ & $3566(19)$ & $105(8)$ \\
\hline$C(32)$ & $4492(15)$ & $6219(12)$ & $3203(19)$ & $118(9)$ \\
\hline$C(33)$ & $3891(15)$ & $6298(13)$ & $3047(19)$ & $121(10)$ \\
\hline $\mathrm{N}(7)$ & $3724(13)$ & $6654(12)$ & $3245(17)$ & $133(9)$ \\
\hline$C(35)$ & $4153(17)$ & $6884(14)$ & $3650(20)$ & $153(12)$ \\
\hline $\mathrm{P}(2)$ & $12265(4)$ & $5759(3)$ & $5846(6)$ & $118(4)$ \\
\hline $\mathrm{N}(1)$ & $7590(7)$ & $5853(6)$ & $5128(8)$ & $92(6)$ \\
\hline$C(1)$ & $7465(6)$ & $5726(6)$ & $5673(10)$ & $85(7)$ \\
\hline$C(2)$ & $8009(8)$ & $5627(6)$ & $6147(8)$ & $76(6)$ \\
\hline$C(4)$ & $8470(6)$ & $5693(6)$ & $5894(10)$ & $83(7)$ \\
\hline$C\left(4^{\prime}\right)$ & $8212(8)$ & $5833(6)$ & $5264(9)$ & $82(6)$ \\
\hline $\mathrm{P}(3)$ & $8330(7)$ & $7402(4)$ & $65(7)$ & $156(5)$ \\
\hline$C(13)$ & $5808(7)$ & $6451(6)$ & 3392(9) & $76(6)$ \\
\hline$C(3)$ & $5593(6)$ & $6646(7)$ & $2798(10)$ & $95(8)$ \\
\hline$C(11)$ & 6080(9) & $6714(7)$ & $2584(8)$ & $94(8)$ \\
\hline$C\left(3^{\prime}\right)$ & $6596(7)$ & $6560(6)$ & $3047(10)$ & $79(6)$ \\
\hline $\mathrm{N}(3)$ & $6427(7)$ & $6397(5)$ & $3546(8)$ & $73(5)$ \\
\hline$C(5)$ & $8552(12)$ & $5945(8)$ & $4904(16)$ & $81(6)$ \\
\hline $\mathrm{N}(2)$ & $7717(7)$ & $6223(6)$ & 3999(9) & $79(5)$ \\
\hline$C(6)$ & $8320(8)$ & $6119(7)$ & $4300(9)$ & $91(7)$ \\
\hline$C(7)$ & $8642(6)$ & $6242(7)$ & $3903(11)$ & $99(8)$ \\
\hline$C(8)$ & $8237(8)$ & $6422(6)$ & $3356(9)$ & $86(7)$ \\
\hline C(9) & 7666(7) & $6410(6)$ & $3415(9)$ & $81(7)$ \\
\hline$C(10)$ & $7149(13)$ & $6555(9)$ & $2988(16)$ & $91(7)$ \\
\hline$C(14)$ & $5528(12)$ & $6340(9)$ & $3760(15)$ & $82(7)$ \\
\hline $\mathrm{N}(4)$ & 6333(7) & $6041(6)$ & 4657(9) & $85(6)$ \\
\hline$C(16)$ & $5732(8)$ & $6151(6)$ & $4362(8)$ & $83(7)$ \\
\hline$C(17)$ & $5416(6)$ & $6041(6)$ & $4772(10)$ & $80(7)$ \\
\hline$C(18)$ & $5822(8)$ & $5864(6)$ & $5320(8)$ & $76(7)$ \\
\hline$C(19)$ & $6389(7)$ & $5864(6)$ & $5249(9)$ & $82(7)$ \\
\hline$C(21)$ & $9180(12)$ & $5890(8)$ & $5118(15)$ & $76(7)$ \\
\hline$C(22)$ & $9434(13)$ & $5513(8)$ & $5232(15)$ & $83(8)$ \\
\hline$C(23)$ & $10027(11)$ & $5454(7)$ & $5472(13)$ & $61(7)$ \\
\hline$C(24)$ & $10169(12)$ & $6134(7)$ & $5516(14)$ & $67(7)$ \\
\hline$C(25)$ & $9561(12)$ & $6201(9)$ & $5268(15)$ & $83(7)$ \\
\hline $\mathrm{C}(41)$ & $10723(15)$ & $5746(12)$ & 4433(19) & $92(8)$ \\
\hline
\end{tabular}




\begin{tabular}{|c|c|c|c|c|}
\hline$C(42)$ & $10358(14)$ & $5954(11)$ & $3887(18)$ & $90(7)$ \\
\hline $\mathrm{C}(43)$ & $10256(12)$ & $6374(10)$ & $3935(16)$ & $90(7)$ \\
\hline $\mathrm{C}(44)$ & $9597(10)$ & $6379(5)$ & $2847(12)$ & $93(7)$ \\
\hline$C(45)$ & 9869(8) & $6578(7)$ & $3413(10)$ & $92(7)$ \\
\hline$C(48)$ & $9762(9)$ & $6981(7)$ & 3463(9) & $88(7)$ \\
\hline C(49) & $9382(10)$ & $7184(5)$ & $2947(12)$ & 119(9) \\
\hline $\mathrm{C}(51)$ & $9110(9)$ & $6985(7)$ & $2381(10)$ & $102(8)$ \\
\hline$C(52)$ & $9218(9)$ & $6583(7)$ & 2331(9) & $103(8)$ \\
\hline$C(46)$ & $9652(14)$ & $5960(10)$ & $2799(17)$ & $95(7)$ \\
\hline $\mathrm{C}(47)$ & $10059(16)$ & $5748(12)$ & $3337(19)$ & $113(9)$ \\
\hline$C(55)$ & $3886(18)$ & $5607(15)$ & $4740(20)$ & $139(10)$ \\
\hline$C(57)$ & $3190(20)$ & $6070(20)$ & $4010(30)$ & $146(11)$ \\
\hline$C(58)$ & $3887(19)$ & $6255(16)$ & $5190(20)$ & $148(11)$ \\
\hline C(59) & $3675(17)$ & $5986(16)$ & $4710(20)$ & $130(9)$ \\
\hline$C(60)$ & $4285(10)$ & $5464(10)$ & $5327(11)$ & $128(9)$ \\
\hline$C(61)$ & $4482(12)$ & 5072(9) & $5406(12)$ & $142(10)$ \\
\hline$C(62)$ & $4888(12)$ & $4951(6)$ & $5980(14)$ & $132(10)$ \\
\hline$C(63)$ & $5097(9)$ & $5221(9)$ & $6473(10)$ & $105(8)$ \\
\hline$C(56)$ & $4900(11)$ & $5613(8)$ & $6394(12)$ & $106(8)$ \\
\hline$C\left(56^{\prime}\right)$ & 4494(11) & $5734(7)$ & $5821(15)$ & $123(9)$ \\
\hline$C(65)$ & $11822(17)$ & $5084(12)$ & $7020(20)$ & $128(10)$ \\
\hline$C(66)$ & $11570(20)$ & 4771(14) & $6640(20)$ & $168(17)$ \\
\hline$C(67)$ & $10812(14)$ & $5521(15)$ & $7030(20)$ & $168(12)$ \\
\hline$C(71)$ & $12810(14)$ & $5513(13)$ & $6450(20)$ & $155(11)$ \\
\hline$C(72)$ & $13459(15)$ & $5528(14)$ & $6470(30)$ & $185(18)$ \\
\hline$C(73)$ & $12240(19)$ & $5220(15)$ & $5030(30)$ & $184(16)$ \\
\hline$C(75)$ & $12520(20)$ & $6325(14)$ & $6010(40)$ & $230(20)$ \\
\hline$C(76)$ & $12210(20)$ & $6609(14)$ & $5720(40)$ & $230(20)$ \\
\hline $\mathrm{C}(77)$ & $8840(20)$ & $7775(13)$ & $510(20)$ & $160(10)$ \\
\hline$C(78)$ & $8592(19)$ & 8141(13) & $680(30)$ & $172(16)$ \\
\hline C(79) & $8860(20)$ & $7002(14)$ & $140(20)$ & $169(12)$ \\
\hline$C(200)$ & $6918(13)$ & $5727(9)$ & $5708(17)$ & 93(7) \\
\hline$C(304)$ & $6980(30)$ & $8163(17)$ & $550(30)$ & 219(19) \\
\hline$C(303)$ & $6810(20)$ & $8015(18)$ & $-90(30)$ & 208(13) \\
\hline C(307) & $1650(30)$ & $6080(30)$ & $3310(40)$ & $390(30)$ \\
\hline$C(306)$ & $1680(30)$ & $6480(20)$ & $3670(30)$ & $360(20)$ \\
\hline $\mathrm{O}(305)$ & $5715(10)$ & $5266(8)$ & $7593(12)$ & $125(7)$ \\
\hline$C(302)$ & $8580(20)$ & $7631(17)$ & $-1050(30)$ & $220(20)$ \\
\hline$C(301)$ & $8150(20)$ & $7594(14)$ & $-630(20)$ & 171(11) \\
\hline$C(308)$ & $1350(20)$ & $6910(30)$ & $2500(30)$ & $360(20)$ \\
\hline$C(311)$ & $2390(30)$ & $7660(20)$ & $2310(40)$ & $303(19)$ \\
\hline$C(313)$ & $11910(20)$ & $5911(14)$ & $7410(20)$ & $164(12)$ \\
\hline$C(314)$ & $8695(18)$ & $7239(14)$ & $1910(20)$ & 121(9) \\
\hline
\end{tabular}




\begin{tabular}{|c|c|c|c|c|}
\hline$C(315)$ & $4316(17)$ & $6143(13)$ & $5800(20)$ & $133(10)$ \\
\hline$C(316)$ & $5529(17)$ & $5070(14)$ & $7060(20)$ & $104(8)$ \\
\hline$C(317)$ & 12319(19) & $5645(14)$ & $5110(20)$ & $157(11)$ \\
\hline$C(318)$ & $11650(30)$ & $6355(12)$ & $7320(30)$ & $250(30)$ \\
\hline$C(319)$ & $10240(20)$ & $5760(20)$ & $6910(40)$ & $310(30)$ \\
\hline $\mathrm{C}(4 \mathrm{~S})$ & $2200(30)$ & $7030(20)$ & $1540(30)$ & $298(18)$ \\
\hline $\mathrm{C}(8 \mathrm{~S})$ & $3460(30)$ & $7620(20)$ & $1780(40)$ & $340(30)$ \\
\hline $\mathrm{C}(10 \mathrm{~S})$ & $2810(30)$ & $7850(20)$ & $2920(40)$ & $320(30)$ \\
\hline$C(12 S)$ & $5280(20)$ & $5310(20)$ & $8690(30)$ & $250(16)$ \\
\hline$C(14 S)$ & $5920(20)$ & $4510(10)$ & $8790(30)$ & $269(16)$ \\
\hline$C(18 S)$ & $3430(20)$ & $7270(20)$ & $2210(30)$ & $288(18)$ \\
\hline$C(19 S)$ & $2370(30)$ & $7260(30)$ & $4370(30)$ & $450(40)$ \\
\hline$C(20 S)$ & $6620(30)$ & $7000(20)$ & $-850(30)$ & $270(20)$ \\
\hline$C(29 S)$ & $6390(30)$ & $5030(20)$ & $9730(30)$ & $264(16)$ \\
\hline$C(34 S)$ & $5950(30)$ & $4880(30)$ & $10060(30)$ & $330(30)$ \\
\hline$C(35 S)$ & $4840(20)$ & $5110(20)$ & $8990(30)$ & $310(30)$ \\
\hline$C(36 S)$ & $6430(40)$ & $4205(15)$ & $9010(50)$ & $400(40)$ \\
\hline$C(37 \mathrm{~S})$ & $6540(30)$ & $7450(20)$ & $-800(30)$ & $243(15)$ \\
\hline$C(38 S)$ & $6278(18)$ & $7360(20)$ & $260(20)$ & $224(14)$ \\
\hline$C(39 S)$ & $5639(16)$ & $7510(20)$ & $-50(30)$ & $290(30)$ \\
\hline$C(40 S)$ & $1880(20)$ & $7205(17)$ & $3720(30)$ & $380(20)$ \\
\hline $\mathrm{C}(41 \mathrm{~S})$ & $760(20)$ & $6990(30)$ & $2610(30)$ & $370(30)$ \\
\hline$C(43 S)$ & $2410(40)$ & $6610(20)$ & $1410(40)$ & $350(30)$ \\
\hline $\mathrm{C}(44 \mathrm{~S})$ & $7570(40)$ & $4340(14)$ & $9050(50)$ & $470(40)$ \\
\hline$C(45 S)$ & $7560(30)$ & $5614(15)$ & $9470(30)$ & $340(20)$ \\
\hline$C(46 S)$ & $8050(30)$ & $5390(30)$ & $8750(30)$ & $360(20)$ \\
\hline$C(47 S)$ & $7780(30)$ & $4767(14)$ & $9270(30)$ & $370(20)$ \\
\hline $\mathrm{C}(48 \mathrm{~S})$ & $8650(20)$ & $5520(30)$ & $9230(30)$ & $390(30)$ \\
\hline$C(49 S)$ & $7810(40)$ & $5940(30)$ & $9140(40)$ & $410(40)$ \\
\hline$C(50 S)$ & $8590(30)$ & $6631(15)$ & $-130(30)$ & 199(18) \\
\hline$S(500)$ & $10652(8)$ & 7317(6) & $5641(10)$ & $232(8)$ \\
\hline $\mathrm{O}(501)$ & $10572(18)$ & $7669(9)$ & $5930(18)$ & $286(18)$ \\
\hline $\mathrm{O}(502)$ & $11123(15)$ & $7081(12)$ & $6002(19)$ & $318(19)$ \\
\hline $\mathrm{O}(503)$ & $10155(12)$ & $7112(10)$ & $5271(16)$ & $225(14)$ \\
\hline$C(500)$ & $10932(15)$ & $7505(11)$ & 4981(18) & $288(14)$ \\
\hline $\mathrm{F}(501)$ & $11410(19)$ & $7735(16)$ & $5310(20)$ & $440(30)$ \\
\hline $\mathrm{F}(502)$ & 11071(19) & $7143(13)$ & $4770(20)$ & $368(19)$ \\
\hline $\mathrm{F}(503)$ & $10440(20)$ & $7703(13)$ & $4582(18)$ & $380(20)$ \\
\hline$S(501)$ & $6390(20)$ & $6855(10)$ & $7370(20)$ & $590(30)$ \\
\hline $\mathrm{O}(504)$ & $6740(30)$ & $6817(15)$ & $6990(30)$ & $600(40)$ \\
\hline $\mathrm{O}(505)$ & $6220(30)$ & $6505(12)$ & $7600(30)$ & $640(40)$ \\
\hline $\mathrm{O}(506)$ & $6490(30)$ & $7187(14)$ & $7760(30)$ & $580(40)$ \\
\hline $\mathrm{C}(501)$ & $5640(30)$ & $7003(16)$ & $6760(30)$ & $650(30)$ \\
\hline
\end{tabular}




\begin{tabular}{lrrrr}
$\mathrm{F}(504)$ & $5490(30)$ & $6650(20)$ & $6430(40)$ & $720(40)$ \\
$\mathrm{F}(505)$ & $5330(30)$ & $7090(20)$ & $7180(40)$ & $730(40)$ \\
$\mathrm{F}(506)$ & $5810(30)$ & $7320(17)$ & $6470(30)$ & $630(40)$ \\
$\mathrm{S}(502)$ & $9350(30)$ & $5084(15)$ & $7750(30)$ & $750(40)$ \\
$\mathrm{O}(507)$ & $8970(40)$ & $5320(20)$ & $7950(40)$ & $700(40)$ \\
$\mathrm{O}(508)$ & $9500(40)$ & $4713(16)$ & $8030(40)$ & $720(40)$ \\
$\mathrm{O}(509)$ & $9310(40)$ & $5110(20)$ & $7110(30)$ & $710(50)$ \\
$\mathrm{C}(502)$ & $10080(30)$ & $5370(20)$ & $8130(40)$ & $770(40)$ \\
$\mathrm{F}(507)$ & $10210(40)$ & $5250(30)$ & $8760(30)$ & $790(50)$ \\
$\mathrm{F}(508)$ & $10420(40)$ & $5210(30)$ & $7790(50)$ & $770(50)$ \\
$\mathrm{F}(509)$ & $9870(50)$ & $5755(17)$ & $7970(50)$ & $820(50)$ \\
$\mathrm{S}(503)$ & $4020(40)$ & $7247(17)$ & $-230(40)$ & $1090(60)$ \\
$\mathrm{O}(510)$ & $3960(60)$ & $7000(20)$ & $250(60)$ & $1060(70)$ \\
$\mathrm{O}(511)$ & $4530(50)$ & $7210(30)$ & $-380(70)$ & $1100(70)$ \\
$\mathrm{O}(512)$ & $3500(50)$ & $7330(30)$ & $-720(60)$ & $1080(70)$ \\
$\mathrm{C}(503)$ & $4160(40)$ & $7752(18)$ & $200(50)$ & $1090(70)$ \\
$\mathrm{F}(510)$ & $4750(50)$ & $7710(20)$ & $580(70)$ & $1050(70)$ \\
$\mathrm{F}(511)$ & $3740(60)$ & $7750(20)$ & $500(70)$ & $1060(70)$ \\
$\mathrm{F}(512)$ & $4060(60)$ & $8010(20)$ & $-310(50)$ & $1140(70)$ \\
\hline
\end{tabular}

Table 8. Bond lengths $[\AA]$ and angles $\left[^{\circ}\right]$ for BT4.

\begin{tabular}{lr}
\hline $\operatorname{Pt}(1)-\mathrm{O}(1)$ & $2.01(2)$ \\
$\operatorname{Pt}(1)-\mathrm{N}(5)$ & $2.16(2)$ \\
$\operatorname{Pt}(1)-\mathrm{P}(1)$ & $2.207(12)$ \\
$\operatorname{Pt}(1)-\mathrm{P}(2)$ & $2.264(9)$ \\
$\operatorname{Pt}(2)-\mathrm{N}(6)$ & $2.088(15)$ \\
$\operatorname{Pt}(2)-\mathrm{O}(3)$ & $2.11(3)$ \\
$\operatorname{Pt}(2)-\mathrm{P}(4)$ & $2.194(15)$ \\
$\operatorname{Pt}(2)-\mathrm{P}(3)$ & $2.302(12)$ \\
$\operatorname{Pt}(3)-\mathrm{N}(7)$ & $2.10(3)$ \\
$\operatorname{Pt}(3)-\mathrm{O}(5)$ & $2.15(3)$ \\
$\operatorname{Pt}(3)-\mathrm{P}(6)$ & $2.236(14)$ \\
$\operatorname{Pt}(3)-\mathrm{P}(5)$ & $2.266(16)$ \\
$\operatorname{Pt}(4)-\mathrm{O}(305)$ & $2.00(3)$ \\
$\operatorname{Pt}(4)-\mathrm{N}(8)$ & $2.100(17)$ \\
$\operatorname{Pt}(4)-\mathrm{P}(8)$ & $2.17(2)$ \\
$\operatorname{Pt}(4)-\mathrm{P}(7)$ & $2.256(14)$ \\
$\mathrm{Zn}(1)-\mathrm{N}(1)$ & $2.072(16)$ \\
$\operatorname{Zn}(1)-\mathrm{N}(2)$ & $2.077(13)$ \\
$\mathrm{Zn}(1)-\mathrm{N}(4)$ & $2.080(13)$ \\
$\mathrm{Zn}(1)-\mathrm{O}(9 \mathrm{~W})$ & $2.11(2)$
\end{tabular}




\begin{tabular}{|c|c|}
\hline $\mathrm{Zn}(1)-\mathrm{N}(3)$ & $2.113(15)$ \\
\hline $\mathrm{P}(4)-\mathrm{C}(37 \mathrm{~S})$ & $1.68(5)$ \\
\hline $\mathrm{P}(4)-\mathrm{C}(38 \mathrm{~S})$ & $1.76(5)$ \\
\hline $\mathrm{P}(4)-\mathrm{C}(303)$ & $1.97(6)$ \\
\hline$P(4)-C(20 S)$ & $2.29(7)$ \\
\hline $\mathrm{P}(5)-\mathrm{C}(4 \mathrm{~S})$ & $1.67(7)$ \\
\hline $\mathrm{P}(5)-\mathrm{C}(18 \mathrm{~S})$ & $1.90(5)$ \\
\hline $\mathrm{P}(5)-\mathrm{C}(311)$ & $1.92(7)$ \\
\hline $\mathrm{P}(6)-\mathrm{C}(308)$ & $1.67(6)$ \\
\hline $\mathrm{P}(6)-\mathrm{C}(306)$ & $1.82(2)$ \\
\hline $\mathrm{P}(6)-\mathrm{C}(40 \mathrm{~S})$ & $1.83(2)$ \\
\hline $\mathrm{P}(7)-\mathrm{C}(29 \mathrm{~S})$ & $1.72(5)$ \\
\hline $\mathrm{P}(7)-\mathrm{C}(14 \mathrm{~S})$ & $1.82(2)$ \\
\hline $\mathrm{P}(7)-\mathrm{C}(12 \mathrm{~S})$ & $1.97(6)$ \\
\hline $\mathrm{P}(8)-\mathrm{C}(46 \mathrm{~S})$ & $1.72(6)$ \\
\hline $\mathrm{P}(8)-\mathrm{C}(47 \mathrm{~S})$ & $1.81(2)$ \\
\hline $\mathrm{P}(8)-\mathrm{C}(45 \mathrm{~S})$ & $1.82(2)$ \\
\hline $\mathrm{O}(1)-\mathrm{C}(41)$ & $1.29(4)$ \\
\hline $\mathrm{O}(2)-\mathrm{C}(41)$ & $1.29(4)$ \\
\hline $\mathrm{O}(3)-\mathrm{C}(314)$ & $1.44(5)$ \\
\hline $\mathrm{O}(4)-\mathrm{C}(314)$ & $1.19(4)$ \\
\hline $\mathrm{O}(5)-\mathrm{C}(57)$ & $1.37(6)$ \\
\hline $\mathrm{O}(6)-\mathrm{C}(57)$ & $1.21(6)$ \\
\hline $\mathrm{O}(8)-\mathrm{C}(316)$ & $1.30(4)$ \\
\hline $\mathrm{N}(5)-\mathrm{C}(24)$ & $1.31(3)$ \\
\hline $\mathrm{N}(5)-\mathrm{C}(23)$ & $1.32(3)$ \\
\hline$C(29)-C(26)$ & 1.39 \\
\hline$C(29)-C(30)$ & 1.39 \\
\hline $\mathrm{C}(29)-\mathrm{H}(29)$ & 0.95 \\
\hline$C(26)-C(27)$ & 1.39 \\
\hline$C(26)-C(10)$ & $1.50(3)$ \\
\hline$C(27)-C(28)$ & 1.39 \\
\hline $\mathrm{C}(27)-\mathrm{H}(27)$ & 0.95 \\
\hline $\mathrm{C}(28)-\mathrm{N}(6)$ & 1.39 \\
\hline $\mathrm{C}(28)-\mathrm{H}(28)$ & 0.95 \\
\hline $\mathrm{N}(6)-\mathrm{C}(30)$ & 1.39 \\
\hline $\mathrm{C}(30)-\mathrm{H}(500)$ & 0.95 \\
\hline $\mathrm{P}(1)-\mathrm{C}(65)$ & $1.77(4)$ \\
\hline $\mathrm{P}(1)-\mathrm{C}(67)$ & $1.840(19)$ \\
\hline $\mathrm{P}(1)-\mathrm{C}(313)$ & $1.85(4)$ \\
\hline N(8)-C(36) & 1.39 \\
\hline $\mathrm{N}(8)-\mathrm{C}(40)$ & 1.39 \\
\hline$C(36)-C(37)$ & 1.39 \\
\hline
\end{tabular}




\begin{tabular}{|c|c|}
\hline $\mathrm{C}(36)-\mathrm{H}(36)$ & 0.95 \\
\hline C(37)-C(38) & 1.39 \\
\hline $\mathrm{C}(37)-\mathrm{H}(37)$ & 0.95 \\
\hline C(38)-C(39) & 1.39 \\
\hline $\mathrm{C}(38)-\mathrm{C}(200)$ & $1.45(3)$ \\
\hline C(39)-C(40) & 1.39 \\
\hline C(39)-H(39) & 0.95 \\
\hline $\mathrm{C}(40)-\mathrm{H}(40)$ & 0.95 \\
\hline C(34)-C(35) & $1.24(4)$ \\
\hline$C(34)-C(31)$ & $1.49(5)$ \\
\hline C(34)-H(34) & 0.95 \\
\hline$C(31)-C(32)$ & $1.25(4)$ \\
\hline$C(31)-C(14)$ & $1.46(4)$ \\
\hline$C(32)-C(33)$ & $1.39(4)$ \\
\hline $\mathrm{C}(32)-\mathrm{H}(32)$ & 0.95 \\
\hline $\mathrm{C}(33)-\mathrm{N}(7)$ & $1.38(5)$ \\
\hline C(33)-H(33) & 0.95 \\
\hline $\mathrm{N}(7)-\mathrm{C}(35)$ & $1.36(5)$ \\
\hline $\mathrm{C}(35)-\mathrm{H}(35)$ & 0.95 \\
\hline $\mathrm{P}(2)-\mathrm{C}(71)$ & $1.74(4)$ \\
\hline $\mathrm{P}(2)-\mathrm{C}(317)$ & $1.75(5)$ \\
\hline $\mathrm{P}(2)-\mathrm{C}(75)$ & $1.99(5)$ \\
\hline $\mathrm{N}(1)-\mathrm{C}(1)$ & 1.42 \\
\hline $\mathrm{N}(1)-\mathrm{C}\left(4^{\prime}\right)$ & 1.42 \\
\hline$C(1)-C(200)$ & $1.34(3)$ \\
\hline$C(1)-C(2)$ & 1.42 \\
\hline$C(2)-C(4)$ & 1.42 \\
\hline $\mathrm{C}(2)-\mathrm{H}(2)$ & 0.95 \\
\hline$C(4)-C\left(4^{\prime}\right)$ & 1.42 \\
\hline $\mathrm{C}(4)-\mathrm{H}(4)$ & 0.95 \\
\hline$C\left(4^{\prime}\right)-C(5)$ & $1.38(3)$ \\
\hline $\mathrm{P}(3)-\mathrm{C}(301)$ & $1.61(4)$ \\
\hline $\mathrm{P}(3)-\mathrm{C}(77)$ & $1.80(5)$ \\
\hline $\mathrm{P}(3)-\mathrm{C}(79)$ & $1.81(4)$ \\
\hline$C(13)-C(14)$ & $1.28(3)$ \\
\hline C(13)-C(3) & 1.42 \\
\hline $\mathrm{C}(13)-\mathrm{N}(3)$ & 1.42 \\
\hline $\mathrm{C}(3)-\mathrm{C}(11)$ & 1.42 \\
\hline $\mathrm{C}(3)-\mathrm{H}(3)$ & 0.95 \\
\hline$C(11)-C\left(3^{\prime}\right)$ & 1.42 \\
\hline $\mathrm{C}(11)-\mathrm{H}(11)$ & 0.95 \\
\hline$C\left(3^{\prime}\right)-C(10)$ & $1.38(3)$ \\
\hline C(3')-N(3) & 1.42 \\
\hline
\end{tabular}




\begin{tabular}{|c|c|}
\hline$C(5)-C(6)$ & $1.41(3)$ \\
\hline$C(5)-C(21)$ & $1.43(4)$ \\
\hline $\mathrm{N}(2)-\mathrm{C}(9)$ & 1.42 \\
\hline $\mathrm{N}(2)-\mathrm{C}(6)$ & 1.42 \\
\hline$C(6)-C(7)$ & 1.42 \\
\hline $\mathrm{C}(7)-\mathrm{C}(8)$ & 1.42 \\
\hline $\mathrm{C}(7)-\mathrm{H}(7)$ & 0.95 \\
\hline $\mathrm{C}(8)-\mathrm{C}(9)$ & 1.42 \\
\hline $\mathrm{C}(8)-\mathrm{H}(8)$ & 0.95 \\
\hline C(9)-C(10) & $1.38(3)$ \\
\hline$C(14)-C(16)$ & $1.42(3)$ \\
\hline $\mathrm{N}(4)-\mathrm{C}(19)$ & 1.42 \\
\hline $\mathrm{N}(4)-\mathrm{C}(16)$ & 1.42 \\
\hline$C(16)-C(17)$ & 1.42 \\
\hline C(17)-C(18) & 1.42 \\
\hline $\mathrm{C}(17)-\mathrm{H}(17)$ & 0.95 \\
\hline$C(18)-C(19)$ & 1.42 \\
\hline C(18)-H(18) & 0.95 \\
\hline$C(19)-C(200)$ & $1.42(3)$ \\
\hline$C(21)-C(25)$ & $1.36(4)$ \\
\hline$C(21)-C(22)$ & $1.39(4)$ \\
\hline$C(22)-C(23)$ & $1.36(3)$ \\
\hline $\mathrm{C}(22)-\mathrm{H}(22)$ & 0.95 \\
\hline $\mathrm{C}(23)-\mathrm{H}(23)$ & 0.95 \\
\hline$C(24)-C(25)$ & $1.39(3)$ \\
\hline $\mathrm{C}(24)-\mathrm{H}(24)$ & 0.95 \\
\hline $\mathrm{C}(25)-\mathrm{H}(25)$ & 0.95 \\
\hline$C(41)-C(42)$ & $1.43(4)$ \\
\hline$C(42)-C(47)$ & $1.39(5)$ \\
\hline$C(42)-C(43)$ & $1.45(4)$ \\
\hline$C(43)-C(45)$ & $1.41(3)$ \\
\hline $\mathrm{C}(43)-\mathrm{H}(43)$ & 0.95 \\
\hline$C(44)-C(45)$ & 1.39 \\
\hline$C(44)-C(52)$ & 1.39 \\
\hline C(44)-C(46) & $1.42(3)$ \\
\hline$C(45)-C(48)$ & 1.39 \\
\hline C(48)-C(49) & 1.39 \\
\hline $\mathrm{C}(48)-\mathrm{H}(48)$ & 0.95 \\
\hline C(49)-C(51) & 1.39 \\
\hline $\mathrm{C}(49)-\mathrm{H}(49)$ & 0.95 \\
\hline$C(51)-C(52)$ & 1.39 \\
\hline $\mathrm{C}(51)-\mathrm{C}(314)$ & $1.46(4)$ \\
\hline $\mathrm{C}(52)-\mathrm{H}(52)$ & 0.95 \\
\hline
\end{tabular}




\begin{tabular}{|c|c|}
\hline$C(46)-C(47)$ & $1.46(4)$ \\
\hline $\mathrm{C}(46)-\mathrm{H}(46)$ & 0.95 \\
\hline $\mathrm{C}(47)-\mathrm{H}(47)$ & 0.95 \\
\hline $\mathrm{C}(55)-\mathrm{C}(59)$ & $1.36(6)$ \\
\hline$C(55)-C(60)$ & $1.43(4)$ \\
\hline $\mathrm{C}(55)-\mathrm{H}(55)$ & 0.95 \\
\hline C(57)-C(59) & $1.63(7)$ \\
\hline $\mathrm{C}(58)-\mathrm{C}(59)$ & $1.38(6)$ \\
\hline C(58)-C(315) & $1.46(5)$ \\
\hline C(58)-H(58) & 0.95 \\
\hline $\mathrm{C}(60)-\mathrm{C}(61)$ & 1.39 \\
\hline $\mathrm{C}(60)-\mathrm{C}\left(56^{\prime}\right)$ & 1.39 \\
\hline $\mathrm{C}(61)-\mathrm{C}(62)$ & 1.39 \\
\hline $\mathrm{C}(61)-\mathrm{H}(61)$ & 0.95 \\
\hline$C(62)-C(63)$ & 1.39 \\
\hline $\mathrm{C}(62)-\mathrm{H}(62)$ & 0.95 \\
\hline C(63)-C(56) & 1.39 \\
\hline$C(63)-C(316)$ & $1.47(5)$ \\
\hline $\mathrm{C}(56)-\mathrm{C}\left(56^{\prime}\right)$ & 1.39 \\
\hline $\mathrm{C}(56)-\mathrm{H}(56)$ & 0.95 \\
\hline $\mathrm{C}\left(56^{\prime}\right)-\mathrm{C}(315)$ & $1.44(4)$ \\
\hline $\mathrm{C}(65)-\mathrm{C}(66)$ & $1.36(5)$ \\
\hline $\mathrm{C}(65)-\mathrm{H}(65 \mathrm{~A})$ & 0.99 \\
\hline $\mathrm{C}(65)-\mathrm{H}(65 \mathrm{~B})$ & 0.99 \\
\hline $\mathrm{C}(66)-\mathrm{H}(66 \mathrm{~A})$ & 0.98 \\
\hline $\mathrm{C}(66)-\mathrm{H}(66 \mathrm{~B})$ & 0.98 \\
\hline $\mathrm{C}(66)-\mathrm{H}(66 \mathrm{C})$ & 0.98 \\
\hline C(67)-C(319) & $1.54(2)$ \\
\hline $\mathrm{C}(67)-\mathrm{H}(67 \mathrm{~A})$ & 0.99 \\
\hline $\mathrm{C}(67)-\mathrm{H}(67 \mathrm{~B})$ & 0.99 \\
\hline $\mathrm{C}(71)-\mathrm{C}(72)$ & $1.54(4)$ \\
\hline $\mathrm{C}(71)-\mathrm{H}(71 \mathrm{~A})$ & 0.99 \\
\hline $\mathrm{C}(71)-\mathrm{H}(71 \mathrm{~B})$ & 0.99 \\
\hline $\mathrm{C}(72)-\mathrm{H}(72 \mathrm{~A})$ & 0.98 \\
\hline $\mathrm{C}(72)-\mathrm{H}(72 \mathrm{~B})$ & 0.98 \\
\hline $\mathrm{C}(72)-\mathrm{H}(72 \mathrm{C})$ & 0.98 \\
\hline$C(73)-C(317)$ & $1.44(5)$ \\
\hline $\mathrm{C}(73)-\mathrm{H}(73 \mathrm{~A})$ & 0.98 \\
\hline $\mathrm{C}(73)-\mathrm{H}(73 \mathrm{~B})$ & 0.98 \\
\hline $\mathrm{C}(73)-\mathrm{H}(73 \mathrm{C})$ & 0.98 \\
\hline$C(75)-C(76)$ & $1.26(6)$ \\
\hline $\mathrm{C}(75)-\mathrm{H}(75 \mathrm{~A})$ & 0.99 \\
\hline $\mathrm{C}(75)-\mathrm{H}(75 \mathrm{~B})$ & 0.99 \\
\hline
\end{tabular}




\begin{tabular}{|c|c|}
\hline $\mathrm{C}(76)-\mathrm{H}(76 \mathrm{~A})$ & 0.9818 \\
\hline $\mathrm{C}(76)-\mathrm{H}(76 \mathrm{~B})$ & 0.9818 \\
\hline $\mathrm{C}(76)-\mathrm{H}(76 \mathrm{C})$ & 0.9818 \\
\hline $\mathrm{C}(77)-\mathrm{C}(78)$ & $1.47(5)$ \\
\hline $\mathrm{C}(77)-\mathrm{H}(77 \mathrm{~A})$ & 0.99 \\
\hline C(77)-H(77B) & 0.99 \\
\hline $\mathrm{C}(78)-\mathrm{H}(78 \mathrm{~A})$ & 0.98 \\
\hline $\mathrm{C}(78)-\mathrm{H}(78 \mathrm{~B})$ & 0.98 \\
\hline $\mathrm{C}(78)-\mathrm{H}(78 \mathrm{C})$ & 0.98 \\
\hline$C(79)-C(50 S)$ & $1.44(6)$ \\
\hline $\mathrm{C}(79)-\mathrm{H}(79 \mathrm{~A})$ & 0.99 \\
\hline C(79)-H(79B) & 0.99 \\
\hline C(304)-C(303) & $1.44(7)$ \\
\hline C(304)-H(501) & 0.98 \\
\hline $\mathrm{C}(304)-\mathrm{H}(502)$ & 0.98 \\
\hline $\mathrm{C}(304)-\mathrm{H}(30 \mathrm{C})$ & 0.98 \\
\hline $\mathrm{C}(303)-\mathrm{H}(503)$ & 0.99 \\
\hline C(303)-H(504) & 0.99 \\
\hline $\mathrm{C}(307)-\mathrm{C}(306)$ & $1.55(2)$ \\
\hline C(307)-H(30D) & 1.1182 \\
\hline C(307)-H(30E) & 1.1157 \\
\hline $\mathrm{C}(307)-\mathrm{H}(30 \mathrm{~F})$ & 1.1154 \\
\hline $\mathrm{C}(306)-\mathrm{H}(30 \mathrm{G})$ & 0.99 \\
\hline $\mathrm{C}(306)-\mathrm{H}(30 \mathrm{H})$ & 0.99 \\
\hline $\mathrm{O}(305)-\mathrm{C}(316)$ & $1.30(4)$ \\
\hline$C(302)-C(301)$ & $1.60(5)$ \\
\hline $\mathrm{C}(302)-\mathrm{H}(505)$ & 0.9802 \\
\hline $\mathrm{C}(302)-\mathrm{H}(506)$ & 0.9802 \\
\hline $\mathrm{C}(302)-\mathrm{H}(30 \mathrm{I})$ & 0.9802 \\
\hline $\mathrm{C}(301)-\mathrm{H}(30 \mathrm{~J})$ & 0.99 \\
\hline $\mathrm{C}(301)-\mathrm{H}(30 \mathrm{~K})$ & 0.99 \\
\hline C(308)-C(41S) & $1.55(2)$ \\
\hline $\mathrm{C}(308)-\mathrm{H}(30 \mathrm{~L})$ & 0.99 \\
\hline $\mathrm{C}(308)-\mathrm{H}(30 \mathrm{M})$ & 0.99 \\
\hline $\mathrm{C}(311)-\mathrm{C}(10 \mathrm{~S})$ & $1.55(2)$ \\
\hline $\mathrm{C}(311)-\mathrm{H}(31 \mathrm{~A})$ & 0.99 \\
\hline $\mathrm{C}(311)-\mathrm{H}(31 \mathrm{~B})$ & 0.99 \\
\hline C(313)-C(318) & $1.61(6)$ \\
\hline C(313)-H(317) & 0.99 \\
\hline $\mathrm{C}(313)-\mathrm{H}(318)$ & 0.99 \\
\hline $\mathrm{C}(315)-\mathrm{H}(400)$ & 0.95 \\
\hline $\mathrm{C}(317)-\mathrm{H}(315)$ & 0.99 \\
\hline $\mathrm{C}(317)-\mathrm{H}(316)$ & 0.99 \\
\hline
\end{tabular}




\begin{tabular}{|c|c|}
\hline $\mathrm{C}(318)-\mathrm{H}(310)$ & 0.98 \\
\hline $\mathrm{C}(318)-\mathrm{H}(311)$ & 0.98 \\
\hline $\mathrm{C}(318)-\mathrm{H}(312)$ & 0.98 \\
\hline $\mathrm{C}(319)-\mathrm{H}(313)$ & 0.98 \\
\hline $\mathrm{C}(319)-\mathrm{H}(31 \mathrm{E})$ & 0.98 \\
\hline C(319)-H(31F) & 0.98 \\
\hline $\mathrm{C}(4 \mathrm{~S})-\mathrm{C}(43 \mathrm{~S})$ & $1.54(2)$ \\
\hline $\mathrm{C}(4 \mathrm{~S})-\mathrm{H}(4 \mathrm{~S} 1)$ & 0.99 \\
\hline $\mathrm{C}(4 \mathrm{~S})-\mathrm{H}(4 \mathrm{~S} 2)$ & 0.99 \\
\hline $\mathrm{C}(8 \mathrm{~S})-\mathrm{C}(18 \mathrm{~S})$ & $1.55(2)$ \\
\hline $\mathrm{C}(8 \mathrm{~S})-\mathrm{H}(8 \mathrm{~S} 1)$ & 1.0258 \\
\hline $\mathrm{C}(8 \mathrm{~S})-\mathrm{H}(8 \mathrm{~S} 2)$ & 1.026 \\
\hline $\mathrm{C}(8 \mathrm{~S})-\mathrm{H}(8 \mathrm{~S} 3)$ & 1.0253 \\
\hline $\mathrm{C}(10 \mathrm{~S})-\mathrm{H}(10 \mathrm{~A})$ & 0.9944 \\
\hline $\mathrm{C}(10 \mathrm{~S})-\mathrm{H}(10 \mathrm{~B})$ & 0.9947 \\
\hline $\mathrm{C}(10 \mathrm{~S})-\mathrm{H}(10 \mathrm{C})$ & 0.9948 \\
\hline$C(12 S)-C(35 S)$ & $1.55(2)$ \\
\hline $\mathrm{C}(12 \mathrm{~S})-\mathrm{H}(12 \mathrm{~A})$ & 0.99 \\
\hline $\mathrm{C}(12 \mathrm{~S})-\mathrm{H}(12 \mathrm{~B})$ & 0.99 \\
\hline$C(14 S)-C(36 S)$ & $1.55(2)$ \\
\hline $\mathrm{C}(14 \mathrm{~S})-\mathrm{H}(14 \mathrm{~A})$ & 0.99 \\
\hline $\mathrm{C}(14 \mathrm{~S})-\mathrm{H}(14 \mathrm{~B})$ & 0.99 \\
\hline $\mathrm{C}(18 \mathrm{~S})-\mathrm{H}(18 \mathrm{~A})$ & 0.99 \\
\hline $\mathrm{C}(18 \mathrm{~S})-\mathrm{H}(18 \mathrm{~B})$ & 0.99 \\
\hline$C(19 S)-C(40 S)$ & $1.55(2)$ \\
\hline $\mathrm{C}(19 \mathrm{~S})-\mathrm{H}(19 \mathrm{~A})$ & 0.9813 \\
\hline $\mathrm{C}(19 \mathrm{~S})-\mathrm{H}(19 \mathrm{~B})$ & 0.9813 \\
\hline C(19S)-H(19C) & 0.9813 \\
\hline$C(20 S)-C(37 S)$ & $1.54(2)$ \\
\hline $\mathrm{C}(20 \mathrm{~S})-\mathrm{H}(20 \mathrm{~A})$ & 0.98 \\
\hline $\mathrm{C}(20 \mathrm{~S})-\mathrm{H}(20 \mathrm{~B})$ & 0.98 \\
\hline $\mathrm{C}(20 \mathrm{~S})-\mathrm{H}(20 \mathrm{C})$ & 0.98 \\
\hline $\mathrm{C}(29 \mathrm{~S})-\mathrm{C}(34 \mathrm{~S})$ & $1.54(2)$ \\
\hline $\mathrm{C}(29 \mathrm{~S})-\mathrm{H}(29 \mathrm{~A})$ & 0.99 \\
\hline $\mathrm{C}(29 \mathrm{~S})-\mathrm{H}(29 \mathrm{~B})$ & 0.99 \\
\hline $\mathrm{C}(34 \mathrm{~S})-\mathrm{H}(34 \mathrm{~A})$ & 1.0196 \\
\hline $\mathrm{C}(34 \mathrm{~S})-\mathrm{H}(34 \mathrm{~B})$ & 1.0208 \\
\hline $\mathrm{C}(34 \mathrm{~S})-\mathrm{H}(34 \mathrm{C})$ & 1.0179 \\
\hline $\mathrm{C}(35 \mathrm{~S})-\mathrm{H}(35 \mathrm{~A})$ & 0.9807 \\
\hline $\mathrm{C}(35 \mathrm{~S})-\mathrm{H}(35 \mathrm{~B})$ & 0.9806 \\
\hline $\mathrm{C}(35 \mathrm{~S})-\mathrm{H}(35 \mathrm{C})$ & 0.9806 \\
\hline $\mathrm{C}(36 \mathrm{~S})-\mathrm{H}(36 \mathrm{~A})$ & 0.9989 \\
\hline $\mathrm{C}(36 \mathrm{~S})-\mathrm{H}(36 \mathrm{~B})$ & 0.9994 \\
\hline
\end{tabular}




\begin{tabular}{|c|c|}
\hline $\mathrm{C}(36 \mathrm{~S})-\mathrm{H}(36 \mathrm{C})$ & 0.9989 \\
\hline $\mathrm{C}(37 \mathrm{~S})-\mathrm{H}(37 \mathrm{~A})$ & 0.99 \\
\hline $\mathrm{C}(37 \mathrm{~S})-\mathrm{H}(37 \mathrm{~B})$ & 0.99 \\
\hline $\mathrm{C}(38 \mathrm{~S})-\mathrm{C}(39 \mathrm{~S})$ & $1.55(2)$ \\
\hline $\mathrm{C}(38 \mathrm{~S})-\mathrm{H}(38 \mathrm{~A})$ & 0.99 \\
\hline $\mathrm{C}(38 \mathrm{~S})-\mathrm{H}(38 \mathrm{~B})$ & 0.99 \\
\hline $\mathrm{C}(39 \mathrm{~S})-\mathrm{H}(39 \mathrm{~A})$ & 0.98 \\
\hline $\mathrm{C}(39 \mathrm{~S})-\mathrm{H}(39 \mathrm{~B})$ & 0.98 \\
\hline $\mathrm{C}(39 \mathrm{~S})-\mathrm{H}(39 \mathrm{C})$ & 0.98 \\
\hline $\mathrm{C}(40 \mathrm{~S})-\mathrm{H}(40 \mathrm{~A})$ & 0.99 \\
\hline $\mathrm{C}(40 \mathrm{~S})-\mathrm{H}(40 \mathrm{~B})$ & 0.99 \\
\hline $\mathrm{C}(41 \mathrm{~S})-\mathrm{H}(41 \mathrm{~A})$ & 0.9992 \\
\hline $\mathrm{C}(41 \mathrm{~S})-\mathrm{H}(41 \mathrm{~B})$ & 0.9988 \\
\hline $\mathrm{C}(41 \mathrm{~S})-\mathrm{H}(41 \mathrm{C})$ & 0.999 \\
\hline $\mathrm{C}(43 \mathrm{~S})-\mathrm{H}(43 \mathrm{~A})$ & 1.0837 \\
\hline $\mathrm{C}(43 \mathrm{~S})-\mathrm{H}(43 \mathrm{~B})$ & 1.0856 \\
\hline $\mathrm{C}(43 \mathrm{~S})-\mathrm{H}(43 \mathrm{C})$ & 1.0826 \\
\hline$C(44 S)-C(47 S)$ & $1.55(2)$ \\
\hline $\mathrm{C}(44 \mathrm{~S})-\mathrm{H}(44 \mathrm{~A})$ & 0.9814 \\
\hline $\mathrm{C}(44 \mathrm{~S})-\mathrm{H}(44 \mathrm{~B})$ & 0.9814 \\
\hline $\mathrm{C}(44 \mathrm{~S})-\mathrm{H}(44 \mathrm{C})$ & 0.9815 \\
\hline$C(45 S)-C(49 S)$ & $1.54(2)$ \\
\hline $\mathrm{C}(45 \mathrm{~S})-\mathrm{H}(45 \mathrm{~A})$ & 0.99 \\
\hline $\mathrm{C}(45 \mathrm{~S})-\mathrm{H}(45 \mathrm{~B})$ & 0.99 \\
\hline$C(46 S)-C(48 S)$ & $1.54(2)$ \\
\hline $\mathrm{C}(46 \mathrm{~S})-\mathrm{H}(46 \mathrm{~A})$ & 0.99 \\
\hline $\mathrm{C}(46 \mathrm{~S})-\mathrm{H}(46 \mathrm{~B})$ & 0.99 \\
\hline $\mathrm{C}(47 \mathrm{~S})-\mathrm{H}(47 \mathrm{~A})$ & 0.99 \\
\hline $\mathrm{C}(47 \mathrm{~S})-\mathrm{H}(47 \mathrm{~B})$ & 0.99 \\
\hline $\mathrm{C}(48 \mathrm{~S})-\mathrm{H}(48 \mathrm{~A})$ & 0.98 \\
\hline $\mathrm{C}(48 \mathrm{~S})-\mathrm{H}(48 \mathrm{~B})$ & 0.98 \\
\hline $\mathrm{C}(48 \mathrm{~S})-\mathrm{H}(48 \mathrm{C})$ & 0.98 \\
\hline $\mathrm{C}(49 \mathrm{~S})-\mathrm{H}(49 \mathrm{~A})$ & 1.0063 \\
\hline $\mathrm{C}(49 \mathrm{~S})-\mathrm{H}(49 \mathrm{~B})$ & 1.0073 \\
\hline $\mathrm{C}(49 \mathrm{~S})-\mathrm{H}(49 \mathrm{C})$ & 1.0067 \\
\hline $\mathrm{C}(50 \mathrm{~S})-\mathrm{H}(50 \mathrm{~A})$ & 0.98 \\
\hline $\mathrm{C}(50 \mathrm{~S})-\mathrm{H}(50 \mathrm{~B})$ & 0.98 \\
\hline $\mathrm{C}(50 \mathrm{~S})-\mathrm{H}(50 \mathrm{C})$ & 0.98 \\
\hline $\mathrm{S}(500)-\mathrm{O}(503)$ & $1.387(19)$ \\
\hline $\mathrm{S}(500)-\mathrm{O}(501)$ & $1.392(19)$ \\
\hline $\mathrm{S}(500)-\mathrm{O}(502)$ & $1.396(19)$ \\
\hline $\mathrm{S}(500)-\mathrm{C}(500)$ & $1.92(5)$ \\
\hline$C(500)-F(501)$ & $1.375(19)$ \\
\hline
\end{tabular}




\begin{tabular}{|c|c|}
\hline$C(500)-F(502)$ & $1.39(2)$ \\
\hline$C(500)-F(503)$ & $1.40(2)$ \\
\hline $\mathrm{S}(501)-\mathrm{O}(504)$ & $1.391(19)$ \\
\hline $\mathrm{S}(501)-\mathrm{O}(506)$ & $1.393(19)$ \\
\hline $\mathrm{S}(501)-\mathrm{O}(505)$ & $1.40(2)$ \\
\hline$S(501)-C(501)$ & $1.93(5)$ \\
\hline $\mathrm{C}(501)-\mathrm{F}(506)$ & $1.38(2)$ \\
\hline $\mathrm{C}(501)-\mathrm{F}(504)$ & $1.39(2)$ \\
\hline $\mathrm{C}(501)-\mathrm{F}(505)$ & $1.39(2)$ \\
\hline $\mathrm{S}(502)-\mathrm{O}(508)$ & $1.39(2)$ \\
\hline $\mathrm{S}(502)-\mathrm{O}(507)$ & $1.39(2)$ \\
\hline $\mathrm{S}(502)-\mathrm{O}(509)$ & $1.40(2)$ \\
\hline$S(502)-C(502)$ & $1.93(5)$ \\
\hline$C(502)-F(507)$ & $1.39(2)$ \\
\hline$C(502)-F(508)$ & $1.39(2)$ \\
\hline$C(502)-F(509)$ & $1.40(2)$ \\
\hline $\mathrm{S}(503)-\mathrm{O}(510)$ & $1.39(2)$ \\
\hline $\mathrm{S}(503)-\mathrm{O}(512)$ & $1.39(2)$ \\
\hline $\mathrm{S}(503)-\mathrm{O}(511)$ & $1.39(2)$ \\
\hline$S(503)-C(503)$ & $1.92(5)$ \\
\hline$C(503)-F(510)$ & $1.39(2)$ \\
\hline$C(503)-F(511)$ & $1.39(2)$ \\
\hline$C(503)-F(512)$ & $1.39(2)$ \\
\hline $\mathrm{O}(1)-\mathrm{Pt}(1)-\mathrm{N}(5)$ & $82.2(9)$ \\
\hline $\mathrm{O}(1)-\mathrm{Pt}(1)-\mathrm{P}(1)$ & $173.5(6)$ \\
\hline $\mathrm{N}(5)-\mathrm{Pt}(1)-\mathrm{P}(1)$ & $91.9(7)$ \\
\hline $\mathrm{O}(1)-\mathrm{Pt}(1)-\mathrm{P}(2)$ & $88.2(6)$ \\
\hline $\mathrm{N}(5)-\mathrm{Pt}(1)-\mathrm{P}(2)$ & $170.1(7)$ \\
\hline $\mathrm{P}(1)-\mathrm{Pt}(1)-\mathrm{P}(2)$ & $97.6(4)$ \\
\hline $\mathrm{N}(6)-\mathrm{Pt}(2)-\mathrm{O}(3)$ & $81.6(8)$ \\
\hline N(6)-Pt(2)-P(4) & $93.2(7)$ \\
\hline $\mathrm{O}(3)-\mathrm{Pt}(2)-\mathrm{P}(4)$ & $174.8(7)$ \\
\hline $\mathrm{N}(6)-\mathrm{Pt}(2)-\mathrm{P}(3)$ & $167.2(7)$ \\
\hline $\mathrm{O}(3)-\mathrm{Pt}(2)-\mathrm{P}(3)$ & $85.6(7)$ \\
\hline $\mathrm{P}(4)-\mathrm{Pt}(2)-\mathrm{P}(3)$ & $99.6(5)$ \\
\hline $\mathrm{N}(7)-\mathrm{Pt}(3)-\mathrm{O}(5)$ & $80.3(11)$ \\
\hline N(7)-Pt(3)-P(6) & $170.6(11)$ \\
\hline $\mathrm{O}(5)-\mathrm{Pt}(3)-\mathrm{P}(6)$ & $90.6(8)$ \\
\hline N(7)-Pt(3)-P(5) & $92.9(10)$ \\
\hline $\mathrm{O}(5)-\mathrm{Pt}(3)-\mathrm{P}(5)$ & $167.0(12)$ \\
\hline $\mathrm{P}(6)-\mathrm{Pt}(3)-\mathrm{P}(5)$ & $95.6(6)$ \\
\hline $\mathrm{O}(305)-\mathrm{Pt}(4)-\mathrm{N}(8)$ & $81.2(8)$ \\
\hline
\end{tabular}




\begin{tabular}{|c|c|}
\hline $\mathrm{O}(305)-\mathrm{Pt}(4)-\mathrm{P}(8)$ & $172.1(9)$ \\
\hline N(8)-Pt(4)-P(8) & $93.0(7)$ \\
\hline $\mathrm{O}(305)-\mathrm{Pt}(4)-\mathrm{P}(7)$ & $86.7(7)$ \\
\hline N(8)-Pt(4)-P(7) & $167.8(8)$ \\
\hline $\mathrm{P}(8)-\mathrm{Pt}(4)-\mathrm{P}(7)$ & $99.2(6)$ \\
\hline $\mathrm{N}(1)-\mathrm{Zn}(1)-\mathrm{N}(2)$ & $89.7(8)$ \\
\hline $\mathrm{N}(1)-\mathrm{Zn}(1)-\mathrm{N}(4)$ & $89.1(8)$ \\
\hline $\mathrm{N}(2)-\mathrm{Zn}(1)-\mathrm{N}(4)$ & $161.6(8)$ \\
\hline $\mathrm{N}(1)-\mathrm{Zn}(1)-\mathrm{O}(9 \mathrm{~W})$ & $105.5(9)$ \\
\hline $\mathrm{N}(2)-\mathrm{Zn}(1)-\mathrm{O}(9 \mathrm{~W})$ & $95.4(9)$ \\
\hline $\mathrm{N}(4)-\mathrm{Zn}(1)-\mathrm{O}(9 \mathrm{~W})$ & $102.6(9)$ \\
\hline $\mathrm{N}(1)-\mathrm{Zn}(1)-\mathrm{N}(3)$ & $162.3(8)$ \\
\hline $\mathrm{N}(2)-\mathrm{Zn}(1)-\mathrm{N}(3)$ & $90.5(7)$ \\
\hline $\mathrm{N}(4)-\mathrm{Zn}(1)-\mathrm{N}(3)$ & $85.3(7)$ \\
\hline $\mathrm{O}(9 \mathrm{~W})-\mathrm{Zn}(1)-\mathrm{N}(3)$ & $92.1(9)$ \\
\hline $\mathrm{C}(37 \mathrm{~S})-\mathrm{P}(4)-\mathrm{C}(38 \mathrm{~S})$ & $102(3)$ \\
\hline $\mathrm{C}(37 \mathrm{~S})-\mathrm{P}(4)-\mathrm{C}(303)$ & $83(3)$ \\
\hline $\mathrm{C}(38 \mathrm{~S})-\mathrm{P}(4)-\mathrm{C}(303)$ & $97(3)$ \\
\hline C(37S)-P(4)-Pt(2) & $137(2)$ \\
\hline C(38S)-P(4)-Pt(2) & $113.8(15)$ \\
\hline $\mathrm{C}(303)-\mathrm{P}(4)-\mathrm{Pt}(2)$ & $113.7(19)$ \\
\hline $\mathrm{C}(37 \mathrm{~S})-\mathrm{P}(4)-\mathrm{C}(20 \mathrm{~S})$ & 42.3(16) \\
\hline $\mathrm{C}(38 \mathrm{~S})-\mathrm{P}(4)-\mathrm{C}(20 \mathrm{~S})$ & $100(3)$ \\
\hline $\mathrm{C}(303)-\mathrm{P}(4)-\mathrm{C}(20 \mathrm{~S})$ & $125(2)$ \\
\hline $\mathrm{Pt}(2)-\mathrm{P}(4)-\mathrm{C}(20 \mathrm{~S})$ & 106.1(19) \\
\hline$C(4 S)-P(5)-C(18 S)$ & 111(3) \\
\hline $\mathrm{C}(4 \mathrm{~S})-\mathrm{P}(5)-\mathrm{C}(311)$ & $96(3)$ \\
\hline $\mathrm{C}(18 \mathrm{~S})-\mathrm{P}(5)-\mathrm{C}(311)$ & $98(3)$ \\
\hline $\mathrm{C}(4 \mathrm{~S})-\mathrm{P}(5)-\mathrm{Pt}(3)$ & $125(3)$ \\
\hline $\mathrm{C}(18 \mathrm{~S})-\mathrm{P}(5)-\mathrm{Pt}(3)$ & $108.2(16)$ \\
\hline $\mathrm{C}(311)-\mathrm{P}(5)-\mathrm{Pt}(3)$ & $116(2)$ \\
\hline $\mathrm{C}(308)-\mathrm{P}(6)-\mathrm{C}(306)$ & 111(3) \\
\hline $\mathrm{C}(308)-\mathrm{P}(6)-\mathrm{C}(40 \mathrm{~S})$ & $105(4)$ \\
\hline $\mathrm{C}(306)-\mathrm{P}(6)-\mathrm{C}(40 \mathrm{~S})$ & $86(4)$ \\
\hline $\mathrm{C}(308)-\mathrm{P}(6)-\mathrm{Pt}(3)$ & $119.2(18)$ \\
\hline $\mathrm{C}(306)-\mathrm{P}(6)-\mathrm{Pt}(3)$ & $118(2)$ \\
\hline $\mathrm{C}(40 \mathrm{~S})-\mathrm{P}(6)-\mathrm{Pt}(3)$ & $112(2)$ \\
\hline $\mathrm{C}(29 \mathrm{~S})-\mathrm{P}(7)-\mathrm{C}(14 \mathrm{~S})$ & $99(3)$ \\
\hline$C(29 S)-P(7)-C(12 S)$ & $111(3)$ \\
\hline $\mathrm{C}(14 \mathrm{~S})-\mathrm{P}(7)-\mathrm{C}(12 \mathrm{~S})$ & $108(3)$ \\
\hline C(29S)-P(7)-Pt(4) & $124.3(19)$ \\
\hline $\mathrm{C}(14 \mathrm{~S})-\mathrm{P}(7)-\mathrm{Pt}(4)$ & $103(2)$ \\
\hline $\mathrm{C}(12 \mathrm{~S})-\mathrm{P}(7)-\mathrm{Pt}(4)$ & $110.2(16)$ \\
\hline
\end{tabular}




\begin{tabular}{|c|c|}
\hline $\mathrm{C}(46 \mathrm{~S})-\mathrm{P}(8)-\mathrm{C}(47 \mathrm{~S})$ & $93(5)$ \\
\hline $\mathrm{C}(46 \mathrm{~S})-\mathrm{P}(8)-\mathrm{C}(45 \mathrm{~S})$ & $86(4)$ \\
\hline $\mathrm{C}(47 \mathrm{~S})-\mathrm{P}(8)-\mathrm{C}(45 \mathrm{~S})$ & $109(3)$ \\
\hline C(46S)-P(8)-Pt(4) & $129(2)$ \\
\hline C(47S)-P(8)-Pt(4) & $121(2)$ \\
\hline C(45S)-P(8)-Pt(4) & $113(3)$ \\
\hline C(41)-O(1)-Pt(1) & $125(2)$ \\
\hline $\mathrm{C}(314)-\mathrm{O}(3)-\mathrm{Pt}(2)$ & $115(2)$ \\
\hline C(57)-O(5)-Pt(3) & $111(3)$ \\
\hline $\mathrm{C}(24)-\mathrm{N}(5)-\mathrm{C}(23)$ & $123(2)$ \\
\hline C(24)-N(5)-Pt(1) & $112.4(16)$ \\
\hline $\mathrm{C}(23)-\mathrm{N}(5)-\mathrm{Pt}(1)$ & $121.6(16)$ \\
\hline$C(26)-C(29)-C(30)$ & 120 \\
\hline $\mathrm{C}(26)-\mathrm{C}(29)-\mathrm{H}(29)$ & 120 \\
\hline $\mathrm{C}(30)-\mathrm{C}(29)-\mathrm{H}(29)$ & 120 \\
\hline$C(27)-C(26)-C(29)$ & 120 \\
\hline$C(27)-C(26)-C(10)$ & $120(2)$ \\
\hline$C(29)-C(26)-C(10)$ & $120(2)$ \\
\hline$C(26)-C(27)-C(28)$ & 120 \\
\hline $\mathrm{C}(26)-\mathrm{C}(27)-\mathrm{H}(27)$ & 120 \\
\hline $\mathrm{C}(28)-\mathrm{C}(27)-\mathrm{H}(27)$ & 120 \\
\hline $\mathrm{N}(6)-\mathrm{C}(28)-\mathrm{C}(27)$ & 120 \\
\hline $\mathrm{N}(6)-\mathrm{C}(28)-\mathrm{H}(28)$ & 120 \\
\hline $\mathrm{C}(27)-\mathrm{C}(28)-\mathrm{H}(28)$ & 120 \\
\hline $\mathrm{C}(30)-\mathrm{N}(6)-\mathrm{C}(28)$ & 120 \\
\hline $\mathrm{C}(30)-\mathrm{N}(6)-\mathrm{Pt}(2)$ & $116.7(13)$ \\
\hline $\mathrm{C}(28)-\mathrm{N}(6)-\mathrm{Pt}(2)$ & $120.9(14)$ \\
\hline $\mathrm{N}(6)-\mathrm{C}(30)-\mathrm{C}(29)$ & 120 \\
\hline $\mathrm{N}(6)-\mathrm{C}(30)-\mathrm{H}(500)$ & 120 \\
\hline $\mathrm{C}(29)-\mathrm{C}(30)-\mathrm{H}(500)$ & 120 \\
\hline C(65)-P(1)-C(67) & $104(2)$ \\
\hline $\mathrm{C}(65)-\mathrm{P}(1)-\mathrm{C}(313)$ & $106(2)$ \\
\hline $\mathrm{C}(67)-\mathrm{P}(1)-\mathrm{C}(313)$ & $100(2)$ \\
\hline $\mathrm{C}(65)-\mathrm{P}(1)-\mathrm{Pt}(1)$ & $116.0(15)$ \\
\hline C(67)-P(1)-Pt(1) & $114.3(15)$ \\
\hline C(313)-P(1)-Pt(1) & $115.2(17)$ \\
\hline $\mathrm{C}(36)-\mathrm{N}(8)-\mathrm{C}(40)$ & 120 \\
\hline $\mathrm{C}(36)-\mathrm{N}(8)-\mathrm{Pt}(4)$ & $123.2(15)$ \\
\hline $\mathrm{C}(40)-\mathrm{N}(8)-\mathrm{Pt}(4)$ & $115.9(15)$ \\
\hline N(8)-C(36)-C(37) & 120 \\
\hline $\mathrm{N}(8)-\mathrm{C}(36)-\mathrm{H}(36)$ & 120 \\
\hline $\mathrm{C}(37)-\mathrm{C}(36)-\mathrm{H}(36)$ & 120 \\
\hline$C(36)-C(37)-C(38)$ & 120 \\
\hline
\end{tabular}




\begin{tabular}{|c|c|}
\hline $\mathrm{C}(36)-\mathrm{C}(37)-\mathrm{H}(37)$ & 120 \\
\hline $\mathrm{C}(38)-\mathrm{C}(37)-\mathrm{H}(37)$ & 120 \\
\hline C(39)-C(38)-C(37) & 120 \\
\hline$C(39)-C(38)-C(200)$ & $119(2)$ \\
\hline$C(37)-C(38)-C(200)$ & $121(2)$ \\
\hline C(38)-C(39)-C(40) & 120 \\
\hline $\mathrm{C}(38)-\mathrm{C}(39)-\mathrm{H}(39)$ & 120 \\
\hline $\mathrm{C}(40)-\mathrm{C}(39)-\mathrm{H}(39)$ & 120 \\
\hline $\mathrm{C}(39)-\mathrm{C}(40)-\mathrm{N}(8)$ & 120 \\
\hline $\mathrm{C}(39)-\mathrm{C}(40)-\mathrm{H}(40)$ & 120 \\
\hline N(8)-C(40)-H(40) & 120 \\
\hline $\mathrm{C}(35)-\mathrm{C}(34)-\mathrm{C}(31)$ & $124(4)$ \\
\hline $\mathrm{C}(35)-\mathrm{C}(34)-\mathrm{H}(34)$ & 118.2 \\
\hline $\mathrm{C}(31)-\mathrm{C}(34)-\mathrm{H}(34)$ & 118.2 \\
\hline $\mathrm{C}(32)-\mathrm{C}(31)-\mathrm{C}(14)$ & $126(4)$ \\
\hline $\mathrm{C}(32)-\mathrm{C}(31)-\mathrm{C}(34)$ & $114(3)$ \\
\hline $\mathrm{C}(14)-\mathrm{C}(31)-\mathrm{C}(34)$ & $120(3)$ \\
\hline $\mathrm{C}(31)-\mathrm{C}(32)-\mathrm{C}(33)$ & $125(4)$ \\
\hline $\mathrm{C}(31)-\mathrm{C}(32)-\mathrm{H}(32)$ & 117.7 \\
\hline $\mathrm{C}(33)-\mathrm{C}(32)-\mathrm{H}(32)$ & 117.6 \\
\hline $\mathrm{N}(7)-\mathrm{C}(33)-\mathrm{C}(32)$ & $118(4)$ \\
\hline N(7)-C(33)-H(33) & 120.9 \\
\hline $\mathrm{C}(32)-\mathrm{C}(33)-\mathrm{H}(33)$ & 120.9 \\
\hline $\mathrm{C}(35)-\mathrm{N}(7)-\mathrm{C}(33)$ & $118(3)$ \\
\hline C(35)-N(7)-Pt(3) & $122(3)$ \\
\hline C(33)-N(7)-Pt(3) & $118(3)$ \\
\hline $\mathrm{C}(34)-\mathrm{C}(35)-\mathrm{N}(7)$ & $121(4)$ \\
\hline $\mathrm{C}(34)-\mathrm{C}(35)-\mathrm{H}(35)$ & 119.5 \\
\hline N(7)-C(35)-H(35) & 119.5 \\
\hline $\mathrm{C}(71)-\mathrm{P}(2)-\mathrm{C}(317)$ & $111(2)$ \\
\hline C(71)-P(2)-C(75) & $102(3)$ \\
\hline $\mathrm{C}(317)-\mathrm{P}(2)-\mathrm{C}(75)$ & $106(3)$ \\
\hline C(71)-P(2)-Pt(1) & $119.9(13)$ \\
\hline $\mathrm{C}(317)-\mathrm{P}(2)-\mathrm{Pt}(1)$ & $110.7(15)$ \\
\hline C(75)-P(2)-Pt(1) & $106.4(14)$ \\
\hline C(1)-N(1)-C(4') & 108 \\
\hline $\mathrm{C}(1)-\mathrm{N}(1)-\mathrm{Zn}(1)$ & $128.7(11)$ \\
\hline $\mathrm{C}\left(4^{\prime}\right)-\mathrm{N}(1)-\mathrm{Zn}(1)$ & $123.3(11)$ \\
\hline$C(200)-C(1)-C(2)$ & $129(2)$ \\
\hline $\mathrm{C}(200)-\mathrm{C}(1)-\mathrm{N}(1)$ & $123(2)$ \\
\hline $\mathrm{C}(2)-\mathrm{C}(1)-\mathrm{N}(1)$ & 108 \\
\hline$C(1)-C(2)-C(4)$ & 108 \\
\hline $\mathrm{C}(1)-\mathrm{C}(2)-\mathrm{H}(2)$ & 126 \\
\hline
\end{tabular}




\begin{tabular}{|c|c|}
\hline $\mathrm{C}(4)-\mathrm{C}(2)-\mathrm{H}(2)$ & 126 \\
\hline$C\left(4^{\prime}\right)-C(4)-C(2)$ & 108 \\
\hline $\mathrm{C}\left(4^{\prime}\right)-\mathrm{C}(4)-\mathrm{H}(4)$ & 126 \\
\hline $\mathrm{C}(2)-\mathrm{C}(4)-\mathrm{H}(4)$ & 126 \\
\hline$C(5)-C\left(4^{\prime}\right)-C(4)$ & $121.7(19)$ \\
\hline $\mathrm{C}(5)-\mathrm{C}\left(4^{\prime}\right)-\mathrm{N}(1)$ & $130.2(19)$ \\
\hline $\mathrm{C}(4)-\mathrm{C}\left(4^{\prime}\right)-\mathrm{N}(1)$ & 108 \\
\hline $\mathrm{C}(301)-\mathrm{P}(3)-\mathrm{C}(77)$ & $100(2)$ \\
\hline $\mathrm{C}(301)-\mathrm{P}(3)-\mathrm{C}(79)$ & $110(2)$ \\
\hline C(77)-P(3)-C(79) & $98(3)$ \\
\hline $\mathrm{C}(301)-\mathrm{P}(3)-\mathrm{Pt}(2)$ & $129.0(18)$ \\
\hline C(77)-P(3)-Pt(2) & $108.0(15)$ \\
\hline C(79)-P(3)-Pt(2) & $107.6(13)$ \\
\hline$C(14)-C(13)-C(3)$ & $129.4(19)$ \\
\hline $\mathrm{C}(14)-\mathrm{C}(13)-\mathrm{N}(3)$ & $122.5(19)$ \\
\hline $\mathrm{C}(3)-\mathrm{C}(13)-\mathrm{N}(3)$ & 108 \\
\hline$C(13)-C(3)-C(11)$ & 108 \\
\hline $\mathrm{C}(13)-\mathrm{C}(3)-\mathrm{H}(3)$ & 126 \\
\hline $\mathrm{C}(11)-\mathrm{C}(3)-\mathrm{H}(3)$ & 126 \\
\hline $\mathrm{C}(3)-\mathrm{C}(11)-\mathrm{C}\left(3^{\prime}\right)$ & 108 \\
\hline $\mathrm{C}(3)-\mathrm{C}(11)-\mathrm{H}(11)$ & 126 \\
\hline $\mathrm{C}\left(3^{\prime}\right)-\mathrm{C}(11)-\mathrm{H}(11)$ & 126 \\
\hline $\mathrm{C}(10)-\mathrm{C}\left(3^{\prime}\right)-\mathrm{N}(3)$ & $127.4(19)$ \\
\hline$C(10)-C\left(3^{\prime}\right)-C(11)$ & $124.5(19)$ \\
\hline $\mathrm{N}(3)-\mathrm{C}\left(3^{\prime}\right)-\mathrm{C}(11)$ & 108 \\
\hline $\mathrm{C}\left(3^{\prime}\right)-\mathrm{N}(3)-\mathrm{C}(13)$ & 108 \\
\hline $\mathrm{C}\left(3^{\prime}\right)-\mathrm{N}(3)-\mathrm{Zn}(1)$ & $122.2(10)$ \\
\hline $\mathrm{C}(13)-\mathrm{N}(3)-\mathrm{Zn}(1)$ & $128.7(10)$ \\
\hline$C\left(4^{\prime}\right)-C(5)-C(6)$ & $124(2)$ \\
\hline$C\left(4^{\prime}\right)-C(5)-C(21)$ & $122(3)$ \\
\hline$C(6)-C(5)-C(21)$ & $114(2)$ \\
\hline $\mathrm{C}(9)-\mathrm{N}(2)-\mathrm{C}(6)$ & 108 \\
\hline $\mathrm{C}(9)-\mathrm{N}(2)-\mathrm{Zn}(1)$ & $124.6(11)$ \\
\hline $\mathrm{C}(6)-\mathrm{N}(2)-\mathrm{Zn}(1)$ & $126.4(11)$ \\
\hline$C(5)-C(6)-C(7)$ & $127.0(17)$ \\
\hline $\mathrm{C}(5)-\mathrm{C}(6)-\mathrm{N}(2)$ & $124.9(17)$ \\
\hline $\mathrm{C}(7)-\mathrm{C}(6)-\mathrm{N}(2)$ & 108 \\
\hline$C(6)-C(7)-C(8)$ & 108 \\
\hline $\mathrm{C}(6)-\mathrm{C}(7)-\mathrm{H}(7)$ & 126 \\
\hline $\mathrm{C}(8)-\mathrm{C}(7)-\mathrm{H}(7)$ & 126 \\
\hline$C(7)-C(8)-C(9)$ & 108 \\
\hline $\mathrm{C}(7)-\mathrm{C}(8)-\mathrm{H}(8)$ & 126 \\
\hline $\mathrm{C}(9)-\mathrm{C}(8)-\mathrm{H}(8)$ & 126 \\
\hline
\end{tabular}




\begin{tabular}{|c|c|}
\hline $\mathrm{C}(10)-\mathrm{C}(9)-\mathrm{N}(2)$ & $125.3(18)$ \\
\hline $\mathrm{C}(10)-\mathrm{C}(9)-\mathrm{C}(8)$ & $126.7(18)$ \\
\hline $\mathrm{N}(2)-\mathrm{C}(9)-\mathrm{C}(8)$ & 108 \\
\hline$C\left(3^{\prime}\right)-C(10)-C(9)$ & $128(3)$ \\
\hline$C\left(3^{\prime}\right)-C(10)-C(26)$ & $116(2)$ \\
\hline $\mathrm{C}(9)-\mathrm{C}(10)-\mathrm{C}(26)$ & $116(2)$ \\
\hline$C(13)-C(14)-C(16)$ & $131(2)$ \\
\hline $\mathrm{C}(13)-\mathrm{C}(14)-\mathrm{C}(31)$ & $118(3)$ \\
\hline $\mathrm{C}(16)-\mathrm{C}(14)-\mathrm{C}(31)$ & $111(2)$ \\
\hline$C(19)-N(4)-C(16)$ & 108 \\
\hline $\mathrm{C}(19)-\mathrm{N}(4)-\mathrm{Zn}(1)$ & $123.4(11)$ \\
\hline $\mathrm{C}(16)-\mathrm{N}(4)-\mathrm{Zn}(1)$ & $127.9(11)$ \\
\hline$C(17)-C(16)-N(4)$ & 108 \\
\hline$C(17)-C(16)-C(14)$ & $129.9(16)$ \\
\hline N(4)-C(16)-C(14) & $122.1(16)$ \\
\hline$C(18)-C(17)-C(16)$ & 108 \\
\hline $\mathrm{C}(18)-\mathrm{C}(17)-\mathrm{H}(17)$ & 126 \\
\hline $\mathrm{C}(16)-\mathrm{C}(17)-\mathrm{H}(17)$ & 126 \\
\hline$C(19)-C(18)-C(17)$ & 108 \\
\hline $\mathrm{C}(19)-\mathrm{C}(18)-\mathrm{H}(18)$ & 126 \\
\hline $\mathrm{C}(17)-\mathrm{C}(18)-\mathrm{H}(18)$ & 126 \\
\hline $\mathrm{N}(4)-\mathrm{C}(19)-\mathrm{C}(18)$ & 108 \\
\hline $\mathrm{N}(4)-\mathrm{C}(19)-\mathrm{C}(200)$ & $126.6(18)$ \\
\hline$C(18)-C(19)-C(200)$ & $125.3(18)$ \\
\hline$C(25)-C(21)-C(22)$ & $116(3)$ \\
\hline$C(25)-C(21)-C(5)$ & $122(3)$ \\
\hline $\mathrm{C}(22)-\mathrm{C}(21)-\mathrm{C}(5)$ & $122(3)$ \\
\hline$C(23)-C(22)-C(21)$ & $123(3)$ \\
\hline $\mathrm{C}(23)-\mathrm{C}(22)-\mathrm{H}(22)$ & 118.5 \\
\hline $\mathrm{C}(21)-\mathrm{C}(22)-\mathrm{H}(22)$ & 118.5 \\
\hline N(5)-C(23)-C(22) & $118(2)$ \\
\hline N(5)-C(23)-H(23) & 121.2 \\
\hline $\mathrm{C}(22)-\mathrm{C}(23)-\mathrm{H}(23)$ & 121.2 \\
\hline $\mathrm{N}(5)-\mathrm{C}(24)-\mathrm{C}(25)$ & $120(2)$ \\
\hline $\mathrm{N}(5)-\mathrm{C}(24)-\mathrm{H}(24)$ & 120.1 \\
\hline $\mathrm{C}(25)-\mathrm{C}(24)-\mathrm{H}(24)$ & 120.1 \\
\hline$C(21)-C(25)-C(24)$ & $120(3)$ \\
\hline $\mathrm{C}(21)-\mathrm{C}(25)-\mathrm{H}(25)$ & 120 \\
\hline $\mathrm{C}(24)-\mathrm{C}(25)-\mathrm{H}(25)$ & 119.9 \\
\hline $\mathrm{O}(1)-\mathrm{C}(41)-\mathrm{O}(2)$ & $117(3)$ \\
\hline $\mathrm{O}(1)-\mathrm{C}(41)-\mathrm{C}(42)$ & $122(4)$ \\
\hline $\mathrm{O}(2)-\mathrm{C}(41)-\mathrm{C}(42)$ & $121(4)$ \\
\hline $\mathrm{C}(47)-\mathrm{C}(42)-\mathrm{C}(41)$ & $120(4)$ \\
\hline
\end{tabular}




\begin{tabular}{|c|c|}
\hline $\mathrm{C}(47)-\mathrm{C}(42)-\mathrm{C}(43)$ & $120(3)$ \\
\hline$C(41)-C(42)-C(43)$ & $119(4)$ \\
\hline $\mathrm{C}(45)-\mathrm{C}(43)-\mathrm{C}(42)$ & $120(3)$ \\
\hline $\mathrm{C}(45)-\mathrm{C}(43)-\mathrm{H}(43)$ & 120.1 \\
\hline $\mathrm{C}(42)-\mathrm{C}(43)-\mathrm{H}(43)$ & 120.1 \\
\hline $\mathrm{C}(45)-\mathrm{C}(44)-\mathrm{C}(52)$ & 120 \\
\hline$C(45)-C(44)-C(46)$ & $121(2)$ \\
\hline $\mathrm{C}(52)-\mathrm{C}(44)-\mathrm{C}(46)$ & $118(2)$ \\
\hline $\mathrm{C}(48)-\mathrm{C}(45)-\mathrm{C}(44)$ & 120 \\
\hline$C(48)-C(45)-C(43)$ & $120(2)$ \\
\hline $\mathrm{C}(44)-\mathrm{C}(45)-\mathrm{C}(43)$ & $120(2)$ \\
\hline$C(49)-C(48)-C(45)$ & 120 \\
\hline $\mathrm{C}(49)-\mathrm{C}(48)-\mathrm{H}(48)$ & 120 \\
\hline $\mathrm{C}(45)-\mathrm{C}(48)-\mathrm{H}(48)$ & 120 \\
\hline$C(48)-C(49)-C(51)$ & 120 \\
\hline $\mathrm{C}(48)-\mathrm{C}(49)-\mathrm{H}(49)$ & 120 \\
\hline $\mathrm{C}(51)-\mathrm{C}(49)-\mathrm{H}(49)$ & 120 \\
\hline $\mathrm{C}(49)-\mathrm{C}(51)-\mathrm{C}(52)$ & 120 \\
\hline$C(49)-C(51)-C(314)$ & $112(3)$ \\
\hline $\mathrm{C}(52)-\mathrm{C}(51)-\mathrm{C}(314)$ & $128(3)$ \\
\hline$C(51)-C(52)-C(44)$ & 120 \\
\hline $\mathrm{C}(51)-\mathrm{C}(52)-\mathrm{H}(52)$ & 120 \\
\hline $\mathrm{C}(44)-\mathrm{C}(52)-\mathrm{H}(52)$ & 120 \\
\hline$C(44)-C(46)-C(47)$ & $118(3)$ \\
\hline $\mathrm{C}(44)-\mathrm{C}(46)-\mathrm{H}(46)$ & 120.9 \\
\hline $\mathrm{C}(47)-\mathrm{C}(46)-\mathrm{H}(46)$ & 120.8 \\
\hline$C(42)-C(47)-C(46)$ & $120(4)$ \\
\hline $\mathrm{C}(42)-\mathrm{C}(47)-\mathrm{H}(47)$ & 120.1 \\
\hline $\mathrm{C}(46)-\mathrm{C}(47)-\mathrm{H}(47)$ & 120.2 \\
\hline $\mathrm{C}(59)-\mathrm{C}(55)-\mathrm{C}(60)$ & $119(5)$ \\
\hline $\mathrm{C}(59)-\mathrm{C}(55)-\mathrm{H}(55)$ & 120.4 \\
\hline $\mathrm{C}(60)-\mathrm{C}(55)-\mathrm{H}(55)$ & 120.4 \\
\hline $\mathrm{O}(6)-\mathrm{C}(57)-\mathrm{O}(5)$ & $136(6)$ \\
\hline $\mathrm{O}(6)-\mathrm{C}(57)-\mathrm{C}(59)$ & $121(6)$ \\
\hline $\mathrm{O}(5)-\mathrm{C}(57)-\mathrm{C}(59)$ & $101(5)$ \\
\hline $\mathrm{C}(59)-\mathrm{C}(58)-\mathrm{C}(315)$ & $122(5)$ \\
\hline C(59)-C(58)-H(58) & 119 \\
\hline $\mathrm{C}(315)-\mathrm{C}(58)-\mathrm{H}(58)$ & 119 \\
\hline C(55)-C(59)-C(58) & $123(5)$ \\
\hline $\mathrm{C}(55)-\mathrm{C}(59)-\mathrm{C}(57)$ & $111(5)$ \\
\hline $\mathrm{C}(58)-\mathrm{C}(59)-\mathrm{C}(57)$ & $127(5)$ \\
\hline $\mathrm{C}(61)-\mathrm{C}(60)-\mathrm{C}\left(56^{\prime}\right)$ & 120 \\
\hline $\mathrm{C}(61)-\mathrm{C}(60)-\mathrm{C}(55)$ & $122(3)$ \\
\hline
\end{tabular}




\begin{tabular}{|c|c|}
\hline$C\left(56^{\prime}\right)-C(60)-C(55)$ & $118(3)$ \\
\hline$C(60)-C(61)-C(62)$ & 120 \\
\hline $\mathrm{C}(60)-\mathrm{C}(61)-\mathrm{H}(61)$ & 120 \\
\hline $\mathrm{C}(62)-\mathrm{C}(61)-\mathrm{H}(61)$ & 120 \\
\hline$C(61)-C(62)-C(63)$ & 120 \\
\hline $\mathrm{C}(61)-\mathrm{C}(62)-\mathrm{H}(62)$ & 120 \\
\hline $\mathrm{C}(63)-\mathrm{C}(62)-\mathrm{H}(62)$ & 120 \\
\hline$C(56)-C(63)-C(62)$ & 120 \\
\hline $\mathrm{C}(56)-\mathrm{C}(63)-\mathrm{C}(316)$ & $123(3)$ \\
\hline$C(62)-C(63)-C(316)$ & $117(3)$ \\
\hline $\mathrm{C}(63)-\mathrm{C}(56)-\mathrm{C}\left(56^{\prime}\right)$ & 120 \\
\hline $\mathrm{C}(63)-\mathrm{C}(56)-\mathrm{H}(56)$ & 120 \\
\hline $\mathrm{C}\left(56^{\prime}\right)-\mathrm{C}(56)-\mathrm{H}(56)$ & 120 \\
\hline$C(56)-C\left(56^{\prime}\right)-C(60)$ & 120 \\
\hline $\mathrm{C}(56)-\mathrm{C}\left(56^{\prime}\right)-\mathrm{C}(315)$ & $115(3)$ \\
\hline $\mathrm{C}(60)-\mathrm{C}\left(56^{\prime}\right)-\mathrm{C}(315)$ & $125(3)$ \\
\hline $\mathrm{C}(66)-\mathrm{C}(65)-\mathrm{P}(1)$ & $116(3)$ \\
\hline $\mathrm{C}(66)-\mathrm{C}(65)-\mathrm{H}(65 \mathrm{~A})$ & 108.2 \\
\hline $\mathrm{P}(1)-\mathrm{C}(65)-\mathrm{H}(65 \mathrm{~A})$ & 108.2 \\
\hline $\mathrm{C}(66)-\mathrm{C}(65)-\mathrm{H}(65 \mathrm{~B})$ & 108.1 \\
\hline $\mathrm{P}(1)-\mathrm{C}(65)-\mathrm{H}(65 \mathrm{~B})$ & 108.1 \\
\hline $\mathrm{H}(65 \mathrm{~A})-\mathrm{C}(65)-\mathrm{H}(65 \mathrm{~B})$ & 107.3 \\
\hline $\mathrm{C}(65)-\mathrm{C}(66)-\mathrm{H}(66 \mathrm{~A})$ & 109.5 \\
\hline $\mathrm{C}(65)-\mathrm{C}(66)-\mathrm{H}(66 \mathrm{~B})$ & 109.5 \\
\hline $\mathrm{H}(66 \mathrm{~A})-\mathrm{C}(66)-\mathrm{H}(66 \mathrm{~B})$ & 109.5 \\
\hline C(65)-C(66)-H(66C) & 109.4 \\
\hline $\mathrm{H}(66 \mathrm{~A})-\mathrm{C}(66)-\mathrm{H}(66 \mathrm{C})$ & 109.5 \\
\hline $\mathrm{H}(66 \mathrm{~B})-\mathrm{C}(66)-\mathrm{H}(66 \mathrm{C})$ & 109.5 \\
\hline $\mathrm{C}(319)-\mathrm{C}(67)-\mathrm{P}(1)$ & $137(4)$ \\
\hline $\mathrm{C}(319)-\mathrm{C}(67)-\mathrm{H}(67 \mathrm{~A})$ & 103.1 \\
\hline $\mathrm{P}(1)-\mathrm{C}(67)-\mathrm{H}(67 \mathrm{~A})$ & 102.9 \\
\hline $\mathrm{C}(319)-\mathrm{C}(67)-\mathrm{H}(67 \mathrm{~B})$ & 103 \\
\hline $\mathrm{P}(1)-\mathrm{C}(67)-\mathrm{H}(67 \mathrm{~B})$ & 103 \\
\hline $\mathrm{H}(67 \mathrm{~A})-\mathrm{C}(67)-\mathrm{H}(67 \mathrm{~B})$ & 105.1 \\
\hline $\mathrm{C}(72)-\mathrm{C}(71)-\mathrm{P}(2)$ & $120(3)$ \\
\hline $\mathrm{C}(72)-\mathrm{C}(71)-\mathrm{H}(71 \mathrm{~A})$ & 107.2 \\
\hline $\mathrm{P}(2)-\mathrm{C}(71)-\mathrm{H}(71 \mathrm{~A})$ & 107.3 \\
\hline $\mathrm{C}(72)-\mathrm{C}(71)-\mathrm{H}(71 \mathrm{~B})$ & 107.3 \\
\hline $\mathrm{P}(2)-\mathrm{C}(71)-\mathrm{H}(71 \mathrm{~B})$ & 107.2 \\
\hline $\mathrm{H}(71 \mathrm{~A})-\mathrm{C}(71)-\mathrm{H}(71 \mathrm{~B})$ & 106.9 \\
\hline $\mathrm{C}(71)-\mathrm{C}(72)-\mathrm{H}(72 \mathrm{~A})$ & 109.4 \\
\hline $\mathrm{C}(71)-\mathrm{C}(72)-\mathrm{H}(72 \mathrm{~B})$ & 109.6 \\
\hline $\mathrm{H}(72 \mathrm{~A})-\mathrm{C}(72)-\mathrm{H}(72 \mathrm{~B})$ & 109.5 \\
\hline
\end{tabular}




\begin{tabular}{|c|c|}
\hline $\mathrm{C}(71)-\mathrm{C}(72)-\mathrm{H}(72 \mathrm{C})$ & 109.4 \\
\hline $\mathrm{H}(72 \mathrm{~A})-\mathrm{C}(72)-\mathrm{H}(72 \mathrm{C})$ & 109.5 \\
\hline $\mathrm{H}(72 \mathrm{~B})-\mathrm{C}(72)-\mathrm{H}(72 \mathrm{C})$ & 109.5 \\
\hline $\mathrm{C}(317)-\mathrm{C}(73)-\mathrm{H}(73 \mathrm{~A})$ & 109.5 \\
\hline $\mathrm{C}(317)-\mathrm{C}(73)-\mathrm{H}(73 \mathrm{~B})$ & 109.4 \\
\hline $\mathrm{H}(73 \mathrm{~A})-\mathrm{C}(73)-\mathrm{H}(73 \mathrm{~B})$ & 109.5 \\
\hline $\mathrm{C}(317)-\mathrm{C}(73)-\mathrm{H}(73 \mathrm{C})$ & 109.4 \\
\hline $\mathrm{H}(73 \mathrm{~A})-\mathrm{C}(73)-\mathrm{H}(73 \mathrm{C})$ & 109.5 \\
\hline $\mathrm{H}(73 \mathrm{~B})-\mathrm{C}(73)-\mathrm{H}(73 \mathrm{C})$ & 109.5 \\
\hline $\mathrm{C}(76)-\mathrm{C}(75)-\mathrm{P}(2)$ & $122(5)$ \\
\hline $\mathrm{C}(76)-\mathrm{C}(75)-\mathrm{H}(75 \mathrm{~A})$ & 106.6 \\
\hline $\mathrm{P}(2)-\mathrm{C}(75)-\mathrm{H}(75 \mathrm{~A})$ & 106.8 \\
\hline $\mathrm{C}(76)-\mathrm{C}(75)-\mathrm{H}(75 \mathrm{~B})$ & 106.8 \\
\hline $\mathrm{P}(2)-\mathrm{C}(75)-\mathrm{H}(75 \mathrm{~B})$ & 106.7 \\
\hline $\mathrm{H}(75 \mathrm{~A})-\mathrm{C}(75)-\mathrm{H}(75 \mathrm{~B})$ & 106.6 \\
\hline $\mathrm{C}(75)-\mathrm{C}(76)-\mathrm{H}(76 \mathrm{~A})$ & 109.7 \\
\hline $\mathrm{C}(75)-\mathrm{C}(76)-\mathrm{H}(76 \mathrm{~B})$ & 109.6 \\
\hline $\mathrm{H}(76 \mathrm{~A})-\mathrm{C}(76)-\mathrm{H}(76 \mathrm{~B})$ & 109.2 \\
\hline C(75)-C(76)-H(76C) & 109.8 \\
\hline $\mathrm{H}(76 \mathrm{~A})-\mathrm{C}(76)-\mathrm{H}(76 \mathrm{C})$ & 109.2 \\
\hline $\mathrm{H}(76 \mathrm{~B})-\mathrm{C}(76)-\mathrm{H}(76 \mathrm{C})$ & 109.3 \\
\hline $\mathrm{C}(78)-\mathrm{C}(77)-\mathrm{P}(3)$ & $118(4)$ \\
\hline $\mathrm{C}(78)-\mathrm{C}(77)-\mathrm{H}(77 \mathrm{~A})$ & 107.7 \\
\hline $\mathrm{P}(3)-\mathrm{C}(77)-\mathrm{H}(77 \mathrm{~A})$ & 107.9 \\
\hline $\mathrm{C}(78)-\mathrm{C}(77)-\mathrm{H}(77 \mathrm{~B})$ & 107.9 \\
\hline $\mathrm{P}(3)-\mathrm{C}(77)-\mathrm{H}(77 \mathrm{~B})$ & 107.9 \\
\hline $\mathrm{H}(77 \mathrm{~A})-\mathrm{C}(77)-\mathrm{H}(77 \mathrm{~B})$ & 107.2 \\
\hline C(77)-C(78)-H(78A) & 109.4 \\
\hline $\mathrm{C}(77)-\mathrm{C}(78)-\mathrm{H}(78 \mathrm{~B})$ & 109.5 \\
\hline $\mathrm{H}(78 \mathrm{~A})-\mathrm{C}(78)-\mathrm{H}(78 \mathrm{~B})$ & 109.5 \\
\hline C(77)-C(78)-H(78C) & 109.5 \\
\hline $\mathrm{H}(78 \mathrm{~A})-\mathrm{C}(78)-\mathrm{H}(78 \mathrm{C})$ & 109.5 \\
\hline $\mathrm{H}(78 \mathrm{~B})-\mathrm{C}(78)-\mathrm{H}(78 \mathrm{C})$ & 109.5 \\
\hline $\mathrm{C}(50 \mathrm{~S})-\mathrm{C}(79)-\mathrm{P}(3)$ & $114(4)$ \\
\hline $\mathrm{C}(50 \mathrm{~S})-\mathrm{C}(79)-\mathrm{H}(79 \mathrm{~A})$ & 108.7 \\
\hline $\mathrm{P}(3)-\mathrm{C}(79)-\mathrm{H}(79 \mathrm{~A})$ & 108.7 \\
\hline $\mathrm{C}(50 \mathrm{~S})-\mathrm{C}(79)-\mathrm{H}(79 \mathrm{~B})$ & 108.8 \\
\hline $\mathrm{P}(3)-\mathrm{C}(79)-\mathrm{H}(79 \mathrm{~B})$ & 108.8 \\
\hline $\mathrm{H}(79 \mathrm{~A})-\mathrm{C}(79)-\mathrm{H}(79 \mathrm{~B})$ & 107.6 \\
\hline$C(1)-C(200)-C(19)$ & $128(3)$ \\
\hline$C(1)-C(200)-C(38)$ & $114(3)$ \\
\hline C(19)-C(200)-C(38) & $118(2)$ \\
\hline $\mathrm{C}(303)-\mathrm{C}(304)-\mathrm{H}(501)$ & 109.5 \\
\hline
\end{tabular}




\begin{tabular}{|c|c|}
\hline $\mathrm{C}(303)-\mathrm{C}(304)-\mathrm{H}(502)$ & 109.5 \\
\hline $\mathrm{H}(501)-\mathrm{C}(304)-\mathrm{H}(502)$ & 109.5 \\
\hline $\mathrm{C}(303)-\mathrm{C}(304)-\mathrm{H}(30 \mathrm{C})$ & 109.4 \\
\hline $\mathrm{H}(501)-\mathrm{C}(304)-\mathrm{H}(30 \mathrm{C})$ & 109.5 \\
\hline $\mathrm{H}(502)-\mathrm{C}(304)-\mathrm{H}(30 \mathrm{C})$ & 109.5 \\
\hline C(304)-C(303)-P(4) & $106(3)$ \\
\hline C(304)-C(303)-H(503) & 110.6 \\
\hline $\mathrm{P}(4)-\mathrm{C}(303)-\mathrm{H}(503)$ & 110.6 \\
\hline $\mathrm{C}(304)-\mathrm{C}(303)-\mathrm{H}(504)$ & 110.5 \\
\hline $\mathrm{P}(4)-\mathrm{C}(303)-\mathrm{H}(504)$ & 110.6 \\
\hline H(503)-C(303)-H(504) & 108.7 \\
\hline $\mathrm{C}(306)-\mathrm{C}(307)-\mathrm{H}(30 \mathrm{D})$ & 123 \\
\hline C(306)-C(307)-H(30E) & 122.6 \\
\hline $\mathrm{H}(30 \mathrm{D})-\mathrm{C}(307)-\mathrm{H}(30 \mathrm{E})$ & 93.4 \\
\hline $\mathrm{C}(306)-\mathrm{C}(307)-\mathrm{H}(30 \mathrm{~F})$ & 122.6 \\
\hline $\mathrm{H}(30 \mathrm{D})-\mathrm{C}(307)-\mathrm{H}(30 \mathrm{~F})$ & 93.5 \\
\hline $\mathrm{H}(30 \mathrm{E})-\mathrm{C}(307)-\mathrm{H}(30 \mathrm{~F})$ & 93.6 \\
\hline $\mathrm{C}(307)-\mathrm{C}(306)-\mathrm{P}(6)$ & $101(5)$ \\
\hline $\mathrm{C}(307)-\mathrm{C}(306)-\mathrm{H}(30 \mathrm{G})$ & 111.7 \\
\hline $\mathrm{P}(6)-\mathrm{C}(306)-\mathrm{H}(30 \mathrm{G})$ & 111.5 \\
\hline $\mathrm{C}(307)-\mathrm{C}(306)-\mathrm{H}(30 \mathrm{H})$ & 111.5 \\
\hline $\mathrm{P}(6)-\mathrm{C}(306)-\mathrm{H}(30 \mathrm{H})$ & 111.6 \\
\hline $\mathrm{H}(30 \mathrm{G})-\mathrm{C}(306)-\mathrm{H}(30 \mathrm{H})$ & 109.4 \\
\hline $\mathrm{C}(316)-\mathrm{O}(305)-\mathrm{Pt}(4)$ & $125(3)$ \\
\hline $\mathrm{C}(301)-\mathrm{C}(302)-\mathrm{H}(505)$ & 109.4 \\
\hline C(301)-C(302)-H(506) & 109.4 \\
\hline $\mathrm{H}(505)-\mathrm{C}(302)-\mathrm{H}(506)$ & 109.5 \\
\hline C(301)-C(302)-H(30I) & 109.7 \\
\hline H(505)-C(302)-H(30I) & 109.5 \\
\hline H(506)-C(302)-H(30I) & 109.5 \\
\hline C(302)-C(301)-P(3) & $126(4)$ \\
\hline $\mathrm{C}(302)-\mathrm{C}(301)-\mathrm{H}(30 \mathrm{~J})$ & 105.7 \\
\hline $\mathrm{P}(3)-\mathrm{C}(301)-\mathrm{H}(30 \mathrm{~J})$ & 105.9 \\
\hline $\mathrm{C}(302)-\mathrm{C}(301)-\mathrm{H}(30 \mathrm{~K})$ & 106 \\
\hline $\mathrm{P}(3)-\mathrm{C}(301)-\mathrm{H}(30 \mathrm{~K})$ & 106 \\
\hline $\mathrm{H}(30 \mathrm{~J})-\mathrm{C}(301)-\mathrm{H}(30 \mathrm{~K})$ & 106.2 \\
\hline C(41S)-C(308)-P(6) & $113(4)$ \\
\hline $\mathrm{C}(41 \mathrm{~S})-\mathrm{C}(308)-\mathrm{H}(30 \mathrm{~L})$ & 109 \\
\hline $\mathrm{P}(6)-\mathrm{C}(308)-\mathrm{H}(30 \mathrm{~L})$ & 108.8 \\
\hline $\mathrm{C}(41 \mathrm{~S})-\mathrm{C}(308)-\mathrm{H}(30 \mathrm{M})$ & 108.8 \\
\hline $\mathrm{P}(6)-\mathrm{C}(308)-\mathrm{H}(30 \mathrm{M})$ & 109 \\
\hline $\mathrm{H}(30 \mathrm{~L})-\mathrm{C}(308)-\mathrm{H}(30 \mathrm{M})$ & 107.7 \\
\hline $\mathrm{C}(10 \mathrm{~S})-\mathrm{C}(311)-\mathrm{P}(5)$ & $108(4)$ \\
\hline
\end{tabular}




\begin{tabular}{|c|c|}
\hline $\mathrm{C}(10 \mathrm{~S})-\mathrm{C}(311)-\mathrm{H}(31 \mathrm{~A})$ & 110.2 \\
\hline $\mathrm{P}(5)-\mathrm{C}(311)-\mathrm{H}(31 \mathrm{~A})$ & 110.2 \\
\hline $\mathrm{C}(10 \mathrm{~S})-\mathrm{C}(311)-\mathrm{H}(31 \mathrm{~B})$ & 110 \\
\hline $\mathrm{P}(5)-\mathrm{C}(311)-\mathrm{H}(31 \mathrm{~B})$ & 110.1 \\
\hline $\mathrm{H}(31 \mathrm{~A})-\mathrm{C}(311)-\mathrm{H}(31 \mathrm{~B})$ & 108.5 \\
\hline C(318)-C(313)-P(1) & $114(3)$ \\
\hline $\mathrm{C}(318)-\mathrm{C}(313)-\mathrm{H}(317)$ & 108.7 \\
\hline $\mathrm{P}(1)-\mathrm{C}(313)-\mathrm{H}(317)$ & 108.7 \\
\hline $\mathrm{C}(318)-\mathrm{C}(313)-\mathrm{H}(318)$ & 108.7 \\
\hline $\mathrm{P}(1)-\mathrm{C}(313)-\mathrm{H}(318)$ & 108.8 \\
\hline $\mathrm{H}(317)-\mathrm{C}(313)-\mathrm{H}(318)$ & 107.7 \\
\hline $\mathrm{O}(4)-\mathrm{C}(314)-\mathrm{O}(3)$ & $121(4)$ \\
\hline $\mathrm{O}(4)-\mathrm{C}(314)-\mathrm{C}(51)$ & $131(5)$ \\
\hline $\mathrm{O}(3)-\mathrm{C}(314)-\mathrm{C}(51)$ & $107(4)$ \\
\hline $\mathrm{C}\left(56^{\prime}\right)-\mathrm{C}(315)-\mathrm{C}(58)$ & $113(4)$ \\
\hline $\mathrm{C}\left(56^{\prime}\right)-\mathrm{C}(315)-\mathrm{H}(400)$ & 123.6 \\
\hline $\mathrm{C}(58)-\mathrm{C}(315)-\mathrm{H}(400)$ & 123.6 \\
\hline $\mathrm{O}(8)-\mathrm{C}(316)-\mathrm{O}(305)$ & $112(4)$ \\
\hline $\mathrm{O}(8)-\mathrm{C}(316)-\mathrm{C}(63)$ & $123(4)$ \\
\hline $\mathrm{O}(305)-\mathrm{C}(316)-\mathrm{C}(63)$ & $125(4)$ \\
\hline $\mathrm{C}(73)-\mathrm{C}(317)-\mathrm{P}(2)$ & $106(4)$ \\
\hline $\mathrm{C}(73)-\mathrm{C}(317)-\mathrm{H}(315)$ & 110.5 \\
\hline $\mathrm{P}(2)-\mathrm{C}(317)-\mathrm{H}(315)$ & 110.5 \\
\hline $\mathrm{C}(73)-\mathrm{C}(317)-\mathrm{H}(316)$ & 110.5 \\
\hline $\mathrm{P}(2)-\mathrm{C}(317)-\mathrm{H}(316)$ & 110.5 \\
\hline $\mathrm{H}(315)-\mathrm{C}(317)-\mathrm{H}(316)$ & 108.7 \\
\hline $\mathrm{C}(313)-\mathrm{C}(318)-\mathrm{H}(310)$ & 109.4 \\
\hline $\mathrm{C}(313)-\mathrm{C}(318)-\mathrm{H}(311)$ & 109.6 \\
\hline $\mathrm{H}(310)-\mathrm{C}(318)-\mathrm{H}(311)$ & 109.5 \\
\hline $\mathrm{C}(313)-\mathrm{C}(318)-\mathrm{H}(312)$ & 109.4 \\
\hline $\mathrm{H}(310)-\mathrm{C}(318)-\mathrm{H}(312)$ & 109.5 \\
\hline $\mathrm{H}(311)-\mathrm{C}(318)-\mathrm{H}(312)$ & 109.5 \\
\hline $\mathrm{C}(67)-\mathrm{C}(319)-\mathrm{H}(313)$ & 109.5 \\
\hline C(67)-C(319)-H(31E) & 109.6 \\
\hline $\mathrm{H}(313)-\mathrm{C}(319)-\mathrm{H}(31 \mathrm{E})$ & 109.5 \\
\hline C(67)-C(319)-H(31F) & 109.4 \\
\hline $\mathrm{H}(313)-\mathrm{C}(319)-\mathrm{H}(31 \mathrm{~F})$ & 109.5 \\
\hline $\mathrm{H}(31 \mathrm{E})-\mathrm{C}(319)-\mathrm{H}(31 \mathrm{~F})$ & 109.5 \\
\hline $\mathrm{C}(43 \mathrm{~S})-\mathrm{C}(4 \mathrm{~S})-\mathrm{P}(5)$ & $102(4)$ \\
\hline $\mathrm{C}(43 \mathrm{~S})-\mathrm{C}(4 \mathrm{~S})-\mathrm{H}(4 \mathrm{~S} 1)$ & 111.3 \\
\hline $\mathrm{P}(5)-\mathrm{C}(4 \mathrm{~S})-\mathrm{H}(4 \mathrm{~S} 1)$ & 111.2 \\
\hline $\mathrm{C}(43 \mathrm{~S})-\mathrm{C}(4 \mathrm{~S})-\mathrm{H}(4 \mathrm{~S} 2)$ & 111.5 \\
\hline $\mathrm{P}(5)-\mathrm{C}(4 \mathrm{~S})-\mathrm{H}(4 \mathrm{~S} 2)$ & 111.4 \\
\hline
\end{tabular}




\begin{tabular}{|c|c|}
\hline $\mathrm{H}(4 \mathrm{~S} 1)-\mathrm{C}(4 \mathrm{~S})-\mathrm{H}(4 \mathrm{~S} 2)$ & 109.2 \\
\hline C(18S)-C(8S)-H(8S1) & 114.2 \\
\hline $\mathrm{C}(18 \mathrm{~S})-\mathrm{C}(8 \mathrm{~S})-\mathrm{H}(8 \mathrm{~S} 2)$ & 113.7 \\
\hline H(8S1)-C(8S)-H(8S2) & 104.7 \\
\hline C(18S)-C(8S)-H(8S3) & 113.7 \\
\hline H(8S1)-C(8S)-H(8S3) & 104.7 \\
\hline H(8S2)-C(8S)-H(8S3) & 104.8 \\
\hline $\mathrm{C}(311)-\mathrm{C}(10 \mathrm{~S})-\mathrm{H}(10 \mathrm{~A})$ & 110.7 \\
\hline $\mathrm{C}(311)-\mathrm{C}(10 \mathrm{~S})-\mathrm{H}(10 \mathrm{~B})$ & 110.9 \\
\hline $\mathrm{H}(10 \mathrm{~A})-\mathrm{C}(10 \mathrm{~S})-\mathrm{H}(10 \mathrm{~B})$ & 108.1 \\
\hline $\mathrm{C}(311)-\mathrm{C}(10 \mathrm{~S})-\mathrm{H}(10 \mathrm{C})$ & 111 \\
\hline $\mathrm{H}(10 \mathrm{~A})-\mathrm{C}(10 \mathrm{~S})-\mathrm{H}(10 \mathrm{C})$ & 108 \\
\hline $\mathrm{H}(10 \mathrm{~B})-\mathrm{C}(10 \mathrm{~S})-\mathrm{H}(10 \mathrm{C})$ & 108 \\
\hline C(35S)-C(12S)-P(7) & $114(4)$ \\
\hline $\mathrm{C}(35 \mathrm{~S})-\mathrm{C}(12 \mathrm{~S})-\mathrm{H}(12 \mathrm{~A})$ & 108.3 \\
\hline $\mathrm{P}(7)-\mathrm{C}(12 \mathrm{~S})-\mathrm{H}(12 \mathrm{~A})$ & 108.7 \\
\hline $\mathrm{C}(35 \mathrm{~S})-\mathrm{C}(12 \mathrm{~S})-\mathrm{H}(12 \mathrm{~B})$ & 109.5 \\
\hline $\mathrm{P}(7)-\mathrm{C}(12 \mathrm{~S})-\mathrm{H}(12 \mathrm{~B})$ & 108.7 \\
\hline $\mathrm{H}(12 \mathrm{~A})-\mathrm{C}(12 \mathrm{~S})-\mathrm{H}(12 \mathrm{~B})$ & 107.7 \\
\hline $\mathrm{C}(36 \mathrm{~S})-\mathrm{C}(14 \mathrm{~S})-\mathrm{P}(7)$ & $121(3)$ \\
\hline $\mathrm{C}(36 \mathrm{~S})-\mathrm{C}(14 \mathrm{~S})-\mathrm{H}(14 \mathrm{~A})$ & 107.2 \\
\hline $\mathrm{P}(7)-\mathrm{C}(14 \mathrm{~S})-\mathrm{H}(14 \mathrm{~A})$ & 107 \\
\hline $\mathrm{C}(36 \mathrm{~S})-\mathrm{C}(14 \mathrm{~S})-\mathrm{H}(14 \mathrm{~B})$ & 107 \\
\hline $\mathrm{P}(7)-\mathrm{C}(14 \mathrm{~S})-\mathrm{H}(14 \mathrm{~B})$ & 107.1 \\
\hline $\mathrm{H}(14 \mathrm{~A})-\mathrm{C}(14 \mathrm{~S})-\mathrm{H}(14 \mathrm{~B})$ & 106.8 \\
\hline C(8S)-C(18S)-P(5) & $118(3)$ \\
\hline $\mathrm{C}(8 \mathrm{~S})-\mathrm{C}(18 \mathrm{~S})-\mathrm{H}(18 \mathrm{~A})$ & 107.6 \\
\hline $\mathrm{P}(5)-\mathrm{C}(18 \mathrm{~S})-\mathrm{H}(18 \mathrm{~A})$ & 107.5 \\
\hline $\mathrm{C}(8 \mathrm{~S})-\mathrm{C}(18 \mathrm{~S})-\mathrm{H}(18 \mathrm{~B})$ & 108 \\
\hline $\mathrm{P}(5)-\mathrm{C}(18 \mathrm{~S})-\mathrm{H}(18 \mathrm{~B})$ & 108 \\
\hline $\mathrm{H}(18 \mathrm{~A})-\mathrm{C}(18 \mathrm{~S})-\mathrm{H}(18 \mathrm{~B})$ & 107.1 \\
\hline C(40S)-C(19S)-H(19A) & 109.6 \\
\hline $\mathrm{C}(40 \mathrm{~S})-\mathrm{C}(19 \mathrm{~S})-\mathrm{H}(19 \mathrm{~B})$ & 109.6 \\
\hline $\mathrm{H}(19 \mathrm{~A})-\mathrm{C}(19 \mathrm{~S})-\mathrm{H}(19 \mathrm{~B})$ & 109.3 \\
\hline $\mathrm{C}(40 \mathrm{~S})-\mathrm{C}(19 \mathrm{~S})-\mathrm{H}(19 \mathrm{C})$ & 109.6 \\
\hline $\mathrm{H}(19 \mathrm{~A})-\mathrm{C}(19 \mathrm{~S})-\mathrm{H}(19 \mathrm{C})$ & 109.4 \\
\hline $\mathrm{H}(19 \mathrm{~B})-\mathrm{C}(19 \mathrm{~S})-\mathrm{H}(19 \mathrm{C})$ & 109.3 \\
\hline C(37S)-C(20S)-P(4) & $47(3)$ \\
\hline $\mathrm{C}(37 \mathrm{~S})-\mathrm{C}(20 \mathrm{~S})-\mathrm{H}(20 \mathrm{~A})$ & 109.5 \\
\hline $\mathrm{P}(4)-\mathrm{C}(20 \mathrm{~S})-\mathrm{H}(20 \mathrm{~A})$ & 67.8 \\
\hline $\mathrm{C}(37 \mathrm{~S})-\mathrm{C}(20 \mathrm{~S})-\mathrm{H}(20 \mathrm{~B})$ & 109.5 \\
\hline P(4)-C(20S)-H(20B) & 145.3 \\
\hline $\mathrm{H}(20 \mathrm{~A})-\mathrm{C}(20 \mathrm{~S})-\mathrm{H}(20 \mathrm{~B})$ & 109.5 \\
\hline
\end{tabular}




\begin{tabular}{|c|c|}
\hline $\mathrm{C}(37 \mathrm{~S})-\mathrm{C}(20 \mathrm{~S})-\mathrm{H}(20 \mathrm{C})$ & 109.4 \\
\hline $\mathrm{P}(4)-\mathrm{C}(20 \mathrm{~S})-\mathrm{H}(20 \mathrm{C})$ & 103.5 \\
\hline $\mathrm{H}(20 \mathrm{~A})-\mathrm{C}(20 \mathrm{~S})-\mathrm{H}(20 \mathrm{C})$ & 109.5 \\
\hline $\mathrm{H}(20 \mathrm{~B})-\mathrm{C}(20 \mathrm{~S})-\mathrm{H}(20 \mathrm{C})$ & 109.5 \\
\hline C(34S)-C(29S)-P(7) & 111(4) \\
\hline $\mathrm{C}(34 \mathrm{~S})-\mathrm{C}(29 \mathrm{~S})-\mathrm{H}(29 \mathrm{~A})$ & 109.1 \\
\hline $\mathrm{P}(7)-\mathrm{C}(29 \mathrm{~S})-\mathrm{H}(29 \mathrm{~A})$ & 109.6 \\
\hline $\mathrm{C}(34 \mathrm{~S})-\mathrm{C}(29 \mathrm{~S})-\mathrm{H}(29 \mathrm{~B})$ & 109.5 \\
\hline $\mathrm{P}(7)-\mathrm{C}(29 \mathrm{~S})-\mathrm{H}(29 \mathrm{~B})$ & 109.5 \\
\hline H(29A)-C(29S)-H(29B) & 108.1 \\
\hline C(29S)-C(34S)-H(34A) & 113.4 \\
\hline C(29S)-C(34S)-H(34B) & 113.7 \\
\hline $\mathrm{H}(34 \mathrm{~A})-\mathrm{C}(34 \mathrm{~S})-\mathrm{H}(34 \mathrm{~B})$ & 105.3 \\
\hline C(29S)-C(34S)-H(34C) & 112.9 \\
\hline $\mathrm{H}(34 \mathrm{~A})-\mathrm{C}(34 \mathrm{~S})-\mathrm{H}(34 \mathrm{C})$ & 105.4 \\
\hline $\mathrm{H}(34 \mathrm{~B})-\mathrm{C}(34 \mathrm{~S})-\mathrm{H}(34 \mathrm{C})$ & 105.5 \\
\hline $\mathrm{C}(12 \mathrm{~S})-\mathrm{C}(35 \mathrm{~S})-\mathrm{H}(35 \mathrm{~A})$ & 110.1 \\
\hline $\mathrm{C}(12 \mathrm{~S})-\mathrm{C}(35 \mathrm{~S})-\mathrm{H}(35 \mathrm{~B})$ & 109.7 \\
\hline $\mathrm{H}(35 \mathrm{~A})-\mathrm{C}(35 \mathrm{~S})-\mathrm{H}(35 \mathrm{~B})$ & 109.4 \\
\hline $\mathrm{C}(12 \mathrm{~S})-\mathrm{C}(35 \mathrm{~S})-\mathrm{H}(35 \mathrm{C})$ & 108.9 \\
\hline $\mathrm{H}(35 \mathrm{~A})-\mathrm{C}(35 \mathrm{~S})-\mathrm{H}(35 \mathrm{C})$ & 109.4 \\
\hline $\mathrm{H}(35 \mathrm{~B})-\mathrm{C}(35 \mathrm{~S})-\mathrm{H}(35 \mathrm{C})$ & 109.4 \\
\hline $\mathrm{C}(14 \mathrm{~S})-\mathrm{C}(36 \mathrm{~S})-\mathrm{H}(36 \mathrm{~A})$ & 111.2 \\
\hline $\mathrm{C}(14 \mathrm{~S})-\mathrm{C}(36 \mathrm{~S})-\mathrm{H}(36 \mathrm{~B})$ & 111.5 \\
\hline $\mathrm{H}(36 \mathrm{~A})-\mathrm{C}(36 \mathrm{~S})-\mathrm{H}(36 \mathrm{~B})$ & 107.5 \\
\hline $\mathrm{C}(14 \mathrm{~S})-\mathrm{C}(36 \mathrm{~S})-\mathrm{H}(36 \mathrm{C})$ & 111.2 \\
\hline $\mathrm{H}(36 \mathrm{~A})-\mathrm{C}(36 \mathrm{~S})-\mathrm{H}(36 \mathrm{C})$ & 107.6 \\
\hline $\mathrm{H}(36 \mathrm{~B})-\mathrm{C}(36 \mathrm{~S})-\mathrm{H}(36 \mathrm{C})$ & 107.6 \\
\hline C(20S)-C(37S)-P(4) & $90(4)$ \\
\hline $\mathrm{C}(20 \mathrm{~S})-\mathrm{C}(37 \mathrm{~S})-\mathrm{H}(37 \mathrm{~A})$ & 113.6 \\
\hline $\mathrm{P}(4)-\mathrm{C}(37 \mathrm{~S})-\mathrm{H}(37 \mathrm{~A})$ & 113.6 \\
\hline $\mathrm{C}(20 \mathrm{~S})-\mathrm{C}(37 \mathrm{~S})-\mathrm{H}(37 \mathrm{~B})$ & 113.5 \\
\hline $\mathrm{P}(4)-\mathrm{C}(37 \mathrm{~S})-\mathrm{H}(37 \mathrm{~B})$ & 113.6 \\
\hline $\mathrm{H}(37 \mathrm{~A})-\mathrm{C}(37 \mathrm{~S})-\mathrm{H}(37 \mathrm{~B})$ & 110.9 \\
\hline C(39S)-C(38S)-P(4) & $126(4)$ \\
\hline $\mathrm{C}(39 \mathrm{~S})-\mathrm{C}(38 \mathrm{~S})-\mathrm{H}(38 \mathrm{~A})$ & 105.8 \\
\hline P(4)-C(38S)-H(38A) & 105.9 \\
\hline $\mathrm{C}(39 \mathrm{~S})-\mathrm{C}(38 \mathrm{~S})-\mathrm{H}(38 \mathrm{~B})$ & 105.8 \\
\hline $\mathrm{P}(4)-\mathrm{C}(38 \mathrm{~S})-\mathrm{H}(38 \mathrm{~B})$ & 105.9 \\
\hline $\mathrm{H}(38 \mathrm{~A})-\mathrm{C}(38 \mathrm{~S})-\mathrm{H}(38 \mathrm{~B})$ & 106.2 \\
\hline C(38S)-C(39S)-H(39A) & 109.5 \\
\hline C(38S)-C(39S)-H(39B) & 109.5 \\
\hline $\mathrm{H}(39 \mathrm{~A})-\mathrm{C}(39 \mathrm{~S})-\mathrm{H}(39 \mathrm{~B})$ & 109.5 \\
\hline
\end{tabular}




\begin{tabular}{|c|c|}
\hline C(38S)-C(39S)-H(39C) & 109.4 \\
\hline $\mathrm{H}(39 \mathrm{~A})-\mathrm{C}(39 \mathrm{~S})-\mathrm{H}(39 \mathrm{C})$ & 109.5 \\
\hline H(39B)-C(39S)-H(39C) & 109.5 \\
\hline$C(19 S)-C(40 S)-P(6)$ & $122(3)$ \\
\hline $\mathrm{C}(19 \mathrm{~S})-\mathrm{C}(40 \mathrm{~S})-\mathrm{H}(40 \mathrm{~A})$ & 106.8 \\
\hline $\mathrm{P}(6)-\mathrm{C}(40 \mathrm{~S})-\mathrm{H}(40 \mathrm{~A})$ & 106.8 \\
\hline $\mathrm{C}(19 \mathrm{~S})-\mathrm{C}(40 \mathrm{~S})-\mathrm{H}(40 \mathrm{~B})$ & 106.9 \\
\hline $\mathrm{P}(6)-\mathrm{C}(40 \mathrm{~S})-\mathrm{H}(40 \mathrm{~B})$ & 106.9 \\
\hline $\mathrm{H}(40 \mathrm{~A})-\mathrm{C}(40 \mathrm{~S})-\mathrm{H}(40 \mathrm{~B})$ & 106.7 \\
\hline C(308)-C(41S)-H(41A) & 111.4 \\
\hline C(308)-C(41S)-H(41B) & 111.2 \\
\hline $\mathrm{H}(41 \mathrm{~A})-\mathrm{C}(41 \mathrm{~S})-\mathrm{H}(41 \mathrm{~B})$ & 107.6 \\
\hline $\mathrm{C}(308)-\mathrm{C}(41 \mathrm{~S})-\mathrm{H}(41 \mathrm{C})$ & 111.3 \\
\hline $\mathrm{H}(41 \mathrm{~A})-\mathrm{C}(41 \mathrm{~S})-\mathrm{H}(41 \mathrm{C})$ & 107.5 \\
\hline $\mathrm{H}(41 \mathrm{~B})-\mathrm{C}(41 \mathrm{~S})-\mathrm{H}(41 \mathrm{C})$ & 107.6 \\
\hline $\mathrm{C}(4 \mathrm{~S})-\mathrm{C}(43 \mathrm{~S})-\mathrm{H}(43 \mathrm{~A})$ & 119.6 \\
\hline $\mathrm{C}(4 \mathrm{~S})-\mathrm{C}(43 \mathrm{~S})-\mathrm{H}(43 \mathrm{~B})$ & 119.8 \\
\hline $\mathrm{H}(43 \mathrm{~A})-\mathrm{C}(43 \mathrm{~S})-\mathrm{H}(43 \mathrm{~B})$ & 97.6 \\
\hline $\mathrm{C}(4 \mathrm{~S})-\mathrm{C}(43 \mathrm{~S})-\mathrm{H}(43 \mathrm{C})$ & 119.4 \\
\hline $\mathrm{H}(43 \mathrm{~A})-\mathrm{C}(43 \mathrm{~S})-\mathrm{H}(43 \mathrm{C})$ & 97.8 \\
\hline $\mathrm{H}(43 \mathrm{~B})-\mathrm{C}(43 \mathrm{~S})-\mathrm{H}(43 \mathrm{C})$ & 97.6 \\
\hline $\mathrm{C}(47 \mathrm{~S})-\mathrm{C}(44 \mathrm{~S})-\mathrm{H}(44 \mathrm{~A})$ & 109.5 \\
\hline C(47S)-C(44S)-H(44B) & 109.6 \\
\hline $\mathrm{H}(44 \mathrm{~A})-\mathrm{C}(44 \mathrm{~S})-\mathrm{H}(44 \mathrm{~B})$ & 109.3 \\
\hline C(47S)-C(44S)-H(44C) & 109.7 \\
\hline $\mathrm{H}(44 \mathrm{~A})-\mathrm{C}(44 \mathrm{~S})-\mathrm{H}(44 \mathrm{C})$ & 109.3 \\
\hline $\mathrm{H}(44 \mathrm{~B})-\mathrm{C}(44 \mathrm{~S})-\mathrm{H}(44 \mathrm{C})$ & 109.3 \\
\hline C(49S)-C(45S)-P(8) & $101(6)$ \\
\hline $\mathrm{C}(49 \mathrm{~S})-\mathrm{C}(45 \mathrm{~S})-\mathrm{H}(45 \mathrm{~A})$ & 111.6 \\
\hline $\mathrm{P}(8)-\mathrm{C}(45 \mathrm{~S})-\mathrm{H}(45 \mathrm{~A})$ & 111.6 \\
\hline $\mathrm{C}(49 \mathrm{~S})-\mathrm{C}(45 \mathrm{~S})-\mathrm{H}(45 \mathrm{~B})$ & 111.7 \\
\hline $\mathrm{P}(8)-\mathrm{C}(45 \mathrm{~S})-\mathrm{H}(45 \mathrm{~B})$ & 111.8 \\
\hline $\mathrm{H}(45 \mathrm{~A})-\mathrm{C}(45 \mathrm{~S})-\mathrm{H}(45 \mathrm{~B})$ & 109.5 \\
\hline $\mathrm{C}(48 \mathrm{~S})-\mathrm{C}(46 \mathrm{~S})-\mathrm{P}(8)$ & $130(5)$ \\
\hline $\mathrm{C}(48 \mathrm{~S})-\mathrm{C}(46 \mathrm{~S})-\mathrm{H}(46 \mathrm{~A})$ & 104.9 \\
\hline $\mathrm{P}(8)-\mathrm{C}(46 \mathrm{~S})-\mathrm{H}(46 \mathrm{~A})$ & 104.9 \\
\hline $\mathrm{C}(48 \mathrm{~S})-\mathrm{C}(46 \mathrm{~S})-\mathrm{H}(46 \mathrm{~B})$ & 104.7 \\
\hline $\mathrm{P}(8)-\mathrm{C}(46 \mathrm{~S})-\mathrm{H}(46 \mathrm{~B})$ & 104.7 \\
\hline $\mathrm{H}(46 \mathrm{~A})-\mathrm{C}(46 \mathrm{~S})-\mathrm{H}(46 \mathrm{~B})$ & 105.8 \\
\hline C(44S)-C(47S)-P(8) & $125(3)$ \\
\hline $\mathrm{C}(44 \mathrm{~S})-\mathrm{C}(47 \mathrm{~S})-\mathrm{H}(47 \mathrm{~A})$ & 106 \\
\hline $\mathrm{P}(8)-\mathrm{C}(47 \mathrm{~S})-\mathrm{H}(47 \mathrm{~A})$ & 106.1 \\
\hline $\mathrm{C}(44 \mathrm{~S})-\mathrm{C}(47 \mathrm{~S})-\mathrm{H}(47 \mathrm{~B})$ & 106.2 \\
\hline
\end{tabular}




\begin{tabular}{|c|c|}
\hline $\mathrm{P}(8)-\mathrm{C}(47 \mathrm{~S})-\mathrm{H}(47 \mathrm{~B})$ & 106.1 \\
\hline $\mathrm{H}(47 \mathrm{~A})-\mathrm{C}(47 \mathrm{~S})-\mathrm{H}(47 \mathrm{~B})$ & 106.3 \\
\hline $\mathrm{C}(46 \mathrm{~S})-\mathrm{C}(48 \mathrm{~S})-\mathrm{H}(48 \mathrm{~A})$ & 109.5 \\
\hline $\mathrm{C}(46 \mathrm{~S})-\mathrm{C}(48 \mathrm{~S})-\mathrm{H}(48 \mathrm{~B})$ & 109.5 \\
\hline $\mathrm{H}(48 \mathrm{~A})-\mathrm{C}(48 \mathrm{~S})-\mathrm{H}(48 \mathrm{~B})$ & 109.5 \\
\hline $\mathrm{C}(46 \mathrm{~S})-\mathrm{C}(48 \mathrm{~S})-\mathrm{H}(48 \mathrm{C})$ & 109.3 \\
\hline $\mathrm{H}(48 \mathrm{~A})-\mathrm{C}(48 \mathrm{~S})-\mathrm{H}(48 \mathrm{C})$ & 109.5 \\
\hline $\mathrm{H}(48 \mathrm{~B})-\mathrm{C}(48 \mathrm{~S})-\mathrm{H}(48 \mathrm{C})$ & 109.5 \\
\hline $\mathrm{C}(45 \mathrm{~S})-\mathrm{C}(49 \mathrm{~S})-\mathrm{H}(49 \mathrm{~A})$ & 111.9 \\
\hline C(45S)-C(49S)-H(49B) & 112.3 \\
\hline $\mathrm{H}(49 \mathrm{~A})-\mathrm{C}(49 \mathrm{~S})-\mathrm{H}(49 \mathrm{~B})$ & 106.7 \\
\hline C(45S)-C(49S)-H(49C) & 112 \\
\hline $\mathrm{H}(49 \mathrm{~A})-\mathrm{C}(49 \mathrm{~S})-\mathrm{H}(49 \mathrm{C})$ & 106.8 \\
\hline H(49B)-C(49S)-H(49C) & 106.8 \\
\hline $\mathrm{C}(79)-\mathrm{C}(50 \mathrm{~S})-\mathrm{H}(50 \mathrm{~A})$ & 109.4 \\
\hline $\mathrm{C}(79)-\mathrm{C}(50 \mathrm{~S})-\mathrm{H}(50 \mathrm{~B})$ & 109.5 \\
\hline $\mathrm{H}(50 \mathrm{~A})-\mathrm{C}(50 \mathrm{~S})-\mathrm{H}(50 \mathrm{~B})$ & 109.5 \\
\hline $\mathrm{C}(79)-\mathrm{C}(50 \mathrm{~S})-\mathrm{H}(50 \mathrm{C})$ & 109.5 \\
\hline $\mathrm{H}(50 \mathrm{~A})-\mathrm{C}(50 \mathrm{~S})-\mathrm{H}(50 \mathrm{C})$ & 109.5 \\
\hline $\mathrm{H}(50 \mathrm{~B})-\mathrm{C}(50 \mathrm{~S})-\mathrm{H}(50 \mathrm{C})$ & 109.5 \\
\hline $\mathrm{O}(503)-\mathrm{S}(500)-\mathrm{O}(501)$ & $118(2)$ \\
\hline $\mathrm{O}(503)-\mathrm{S}(500)-\mathrm{O}(502)$ & $116(2)$ \\
\hline $\mathrm{O}(501)-\mathrm{S}(500)-\mathrm{O}(502)$ & $115(2)$ \\
\hline $\mathrm{O}(503)-\mathrm{S}(500)-\mathrm{C}(500)$ & $98.9(18)$ \\
\hline $\mathrm{O}(501)-\mathrm{S}(500)-\mathrm{C}(500)$ & $102.6(19)$ \\
\hline $\mathrm{O}(502)-\mathrm{S}(500)-\mathrm{C}(500)$ & $102.2(19)$ \\
\hline $\mathrm{F}(501)-\mathrm{C}(500)-\mathrm{F}(502)$ & $115(2)$ \\
\hline $\mathrm{F}(501)-\mathrm{C}(500)-\mathrm{F}(503)$ & $117(2)$ \\
\hline $\mathrm{F}(502)-\mathrm{C}(500)-\mathrm{F}(503)$ & $117(2)$ \\
\hline $\mathrm{F}(501)-\mathrm{C}(500)-\mathrm{S}(500)$ & $102(2)$ \\
\hline $\mathrm{F}(502)-\mathrm{C}(500)-\mathrm{S}(500)$ & $99(2)$ \\
\hline $\mathrm{F}(503)-\mathrm{C}(500)-\mathrm{S}(500)$ & $102(2)$ \\
\hline $\mathrm{O}(504)-\mathrm{S}(501)-\mathrm{O}(506)$ & $117(2)$ \\
\hline $\mathrm{O}(504)-\mathrm{S}(501)-\mathrm{O}(505)$ & $117(2)$ \\
\hline $\mathrm{O}(506)-\mathrm{S}(501)-\mathrm{O}(505)$ & $117(2)$ \\
\hline $\mathrm{O}(504)-\mathrm{S}(501)-\mathrm{C}(501)$ & $101(2)$ \\
\hline $\mathrm{O}(506)-\mathrm{S}(501)-\mathrm{C}(501)$ & $99(2)$ \\
\hline $\mathrm{O}(505)-\mathrm{S}(501)-\mathrm{C}(501)$ & $98(2)$ \\
\hline $\mathrm{F}(506)-\mathrm{C}(501)-\mathrm{F}(504)$ & $119(2)$ \\
\hline $\mathrm{F}(506)-\mathrm{C}(501)-\mathrm{F}(505)$ & $117(2)$ \\
\hline $\mathrm{F}(504)-\mathrm{C}(501)-\mathrm{F}(505)$ & $116(2)$ \\
\hline $\mathrm{F}(506)-\mathrm{C}(501)-\mathrm{S}(501)$ & $100(2)$ \\
\hline $\mathrm{F}(504)-\mathrm{C}(501)-\mathrm{S}(501)$ & $100(2)$ \\
\hline
\end{tabular}




\begin{tabular}{lr}
$\mathrm{F}(505)-\mathrm{C}(501)-\mathrm{S}(501)$ & $99(2)$ \\
$\mathrm{O}(508)-\mathrm{S}(502)-\mathrm{O}(507)$ & $118(2)$ \\
$\mathrm{O}(508)-\mathrm{S}(502)-\mathrm{O}(509)$ & $116(2)$ \\
$\mathrm{O}(507)-\mathrm{S}(502)-\mathrm{O}(509)$ & $117(2)$ \\
$\mathrm{O}(508)-\mathrm{S}(502)-\mathrm{C}(502)$ & $100(2)$ \\
$\mathrm{O}(507)-\mathrm{S}(502)-\mathrm{C}(502)$ & $99(2)$ \\
$\mathrm{O}(509)-\mathrm{S}(502)-\mathrm{C}(502)$ & $99(2)$ \\
$\mathrm{F}(507)-\mathrm{C}(502)-\mathrm{F}(508)$ & $118(2)$ \\
$\mathrm{F}(507)-\mathrm{C}(502)-\mathrm{F}(509)$ & $117(2)$ \\
$\mathrm{F}(508)-\mathrm{C}(502)-\mathrm{F}(509)$ & $117(2)$ \\
$\mathrm{F}(507)-\mathrm{C}(502)-\mathrm{S}(502)$ & $100(2)$ \\
$\mathrm{F}(508)-\mathrm{C}(502)-\mathrm{S}(502)$ & $100(2)$ \\
$\mathrm{F}(509)-\mathrm{C}(502)-\mathrm{S}(502)$ & $99(2)$ \\
$\mathrm{O}(510)-\mathrm{S}(503)-\mathrm{O}(512)$ & $117(2)$ \\
$\mathrm{O}(510)-\mathrm{S}(503)-\mathrm{O}(511)$ & $117(2)$ \\
$\mathrm{O}(512)-\mathrm{S}(503)-\mathrm{O}(511)$ & $116(2)$ \\
$\mathrm{O}(510)-\mathrm{S}(503)-\mathrm{C}(503)$ & $101(2)$ \\
$\mathrm{O}(512)-\mathrm{S}(503)-\mathrm{C}(503)$ & $100(2)$ \\
$\mathrm{O}(511)-\mathrm{S}(503)-\mathrm{C}(503)$ & $100(2)$ \\
$\mathrm{F}(510)-\mathrm{C}(503)-\mathrm{F}(511)$ & $117(2)$ \\
$\mathrm{F}(510)-\mathrm{C}(503)-\mathrm{F}(512)$ & $116(2)$ \\
$\mathrm{F}(511)-\mathrm{C}(503)-\mathrm{F}(512)$ & $117(2)$ \\
$\mathrm{F}(510)-\mathrm{C}(503)-\mathrm{S}(503)$ & $101(2)$ \\
$\mathrm{F}(511)-\mathrm{C}(503)-\mathrm{S}(503)$ & $101(2)$ \\
$\mathrm{F}(512)-\mathrm{C}(503)-\mathrm{S}(503)$ & $100(2)$ \\
\hline
\end{tabular}

Table 9. Atomic coordinates ( $\left.\mathrm{x} 10^{4}\right)$ and equivalent isotropic displacement parameters $\left(\AA^{2} \times 10^{3}\right)$ for BT2'. U(eq) is defined as one third of the trace of the orthogonalized $\mathrm{U}^{\mathrm{ij}}$ tensor.

\begin{tabular}{lrrrr}
\hline & $\mathrm{x}$ & $\mathrm{y}$ & \multicolumn{1}{c}{$\mathrm{z}$} & \multicolumn{1}{c}{$\mathrm{U}(\mathrm{eq})$} \\
\hline $\mathrm{Pt}(1)$ & $5816(1)$ & $3577(1)$ & $4430(1)$ & $28(1)$ \\
$\mathrm{Pt}(2)$ & $6648(1)$ & $10841(1)$ & $7624(1)$ & $31(1)$ \\
$\mathrm{S}(1)$ & $5841(1)$ & $9864(3)$ & $4772(2)$ & $56(1)$ \\
$\mathrm{S}(2)$ & $6862(1)$ & $5026(3)$ & $1953(3)$ & $72(2)$ \\
$\mathrm{P}(1)$ & $5391(1)$ & $2790(2)$ & $4385(2)$ & $34(1)$ \\
$\mathrm{P}(2)$ & $6120(1)$ & $2824(2)$ & $4013(2)$ & $34(1)$ \\
$\mathrm{P}(3)$ & $6376(1)$ & $11249(2)$ & $8280(2)$ & $44(1)$ \\
$\mathrm{P}(4)$ & $6983(1)$ & $11847(3)$ & $7697(2)$ & $51(1)$ \\
$\mathrm{F}(1)$ & $5361(5)$ & $9151(11)$ & $4997(14)$ & $198(12)$ \\
$\mathrm{F}(2)$ & $5351(4)$ & $10414(11)$ & $5084(9)$ & $143(8)$ \\
$\mathrm{F}(3)$ & $5690(7)$ & $9795(14)$ & $5788(10)$ & $208(13)$
\end{tabular}




\begin{tabular}{|c|c|c|c|c|}
\hline $\mathrm{F}(4)$ & $7043(4)$ & $4415(7)$ & 2994(7) & $124(7)$ \\
\hline $\mathrm{F}(5)$ & $6475(4)$ & $4441(7)$ & $2572(5)$ & $94(4)$ \\
\hline $\mathrm{F}(6)$ & $6715(3)$ & $5486(6)$ & 2935(4) & $79(4)$ \\
\hline $\mathrm{O}(1)$ & $5589(2)$ & $4297(5)$ & 4910(5) & $37(2)$ \\
\hline $\mathrm{O}(2)$ & $5375(3)$ & $5114(7)$ & $4130(6)$ & $62(4)$ \\
\hline $\mathrm{O}(3)$ & 6341(3) & $9883(6)$ & $7490(5)$ & $40(2)$ \\
\hline $\mathrm{O}(4)$ & $6775(3)$ & $9106(6)$ & $8007(5)$ & $49(3)$ \\
\hline $\mathrm{O}(5)$ & 6032(4) & 10497(7) & $5080(8)$ & $106(7)$ \\
\hline $\mathrm{O}(6)$ & $5652(4)$ & 9994(11) & $4157(6)$ & $110(7)$ \\
\hline $\mathrm{O}(7)$ & 6012(3) & $9150(7)$ & 4900(7) & $77(4)$ \\
\hline $\mathrm{O}(8)$ & 6882(4) & $4286(6)$ & $1720(5)$ & $76(5)$ \\
\hline $\mathrm{O}(9)$ & $6565(3)$ & $5436(10)$ & $1586(6)$ & $82(5)$ \\
\hline $\mathrm{O}(10)$ & $7132(3)$ & $5512(7)$ & $2125(6)$ & $65(4)$ \\
\hline $\mathrm{N}(1)$ & 7384(3) & $6487(5)$ & $4577(5)$ & $24(2)$ \\
\hline $\mathrm{N}(2)$ & 7052(2) & 7543(6) & $5213(4)$ & $21(2)$ \\
\hline $\mathrm{N}(3)$ & $6147(3)$ & $4493(7)$ & $4539(5)$ & $30(3)$ \\
\hline $\mathrm{N}(4)$ & $6837(3)$ & $10267(6)$ & 6993(5) & $26(2)$ \\
\hline$C(1)$ & $7563(3)$ & $6036(7)$ & $4309(6)$ & $27(3)$ \\
\hline$C(2)$ & $7417(4)$ & $5304(8)$ & $4162(7)$ & $35(3)$ \\
\hline$C(3)$ & 7131(4) & $5312(8)$ & 4311(7) & $36(3)$ \\
\hline$C(4)$ & $7108(3)$ & $6066(7)$ & $4572(5)$ & $23(3)$ \\
\hline$C(5)$ & $6850(3)$ & $6281(7)$ & 4797(6) & $28(3)$ \\
\hline$C(6)$ & 6824(3) & 6951(7) & $5093(6)$ & $23(3)$ \\
\hline$C(7)$ & $6564(3)$ & 7184(8) & $5345(7)$ & $34(3)$ \\
\hline$C(8)$ & $6646(3)$ & 7859(7) & $5629(6)$ & $31(3)$ \\
\hline $\mathrm{C}(9)$ & $6956(3)$ & $8109(7)$ & $5545(6)$ & $22(3)$ \\
\hline$C(10)$ & 7131(3) & $8765(7)$ & $5781(6)$ & $26(3)$ \\
\hline $\mathrm{C}(11)$ & 6589(3) & $5705(8)$ & $4721(6)$ & $30(3)$ \\
\hline$C(12)$ & $6365(3)$ & $5563(8)$ & $4146(6)$ & $28(3)$ \\
\hline$C(13)$ & $6146(3)$ & 4972(8) & $4068(6)$ & $28(3)$ \\
\hline$C(14)$ & $6348(3)$ & $4656(8)$ & $5104(6)$ & $33(3)$ \\
\hline$C(15)$ & $6571(3)$ & $5236(8)$ & $5203(6)$ & $30(3)$ \\
\hline$C(16)$ & $7014(3)$ & $9274(7)$ & $6190(6)$ & $24(3)$ \\
\hline$C(17)$ & $6723(3)$ & $9692(7)$ & $6005(6)$ & $26(3)$ \\
\hline$C(18)$ & $6646(3)$ & $10159(7)$ & $6413(6)$ & $31(3)$ \\
\hline$C(19)$ & $7112(3)$ & $9847(8)$ & $7168(6)$ & $32(3)$ \\
\hline$C(20)$ & $7210(3)$ & $9369(8)$ & $6786(6)$ & $28(3)$ \\
\hline $\mathrm{C}(21)$ & $5508(4)$ & $4973(10)$ & 4671(9) & $49(4)$ \\
\hline$C(22)$ & 5612(3) & $5615(8)$ & $5126(7)$ & $34(3)$ \\
\hline$C(23)$ & $5792(4)$ & $5456(9)$ & $5739(8)$ & $47(4)$ \\
\hline$C(24)$ & 5929(4) & 6049(9) & $6139(8)$ & $49(4)$ \\
\hline$C(25)$ & $5869(3)$ & 6809(9) & $5948(7)$ & $35(3)$ \\
\hline$C(26)$ & $5688(3)$ & $6960(9)$ & $5357(7)$ & $40(4)$ \\
\hline
\end{tabular}




\begin{tabular}{|c|c|c|c|c|}
\hline$C(27)$ & 5563(3) & 6372(9) & 4939(7) & $40(4)$ \\
\hline$C(28)$ & 6023(4) & $7410(8)$ & $6385(6)$ & $32(3)$ \\
\hline$C(29)$ & 6341(4) & $7337(8)$ & $6797(7)$ & $37(3)$ \\
\hline$C(30)$ & $6485(4)$ & 7922(8) & $7199(6)$ & $35(3)$ \\
\hline$C(31)$ & 6332(4) & $8582(8)$ & $7182(6)$ & $33(3)$ \\
\hline$C(32)$ & 6023(4) & $8681(9)$ & $6778(8)$ & $45(4)$ \\
\hline$C(33)$ & $5878(3)$ & $8106(10)$ & $6384(7)$ & $43(4)$ \\
\hline$C(34)$ & 6502(4) & $9220(9)$ & 7624(7) & $40(4)$ \\
\hline$C(35)$ & $5362(4)$ & 1841(9) & $4047(7)$ & $40(4)$ \\
\hline$C(36)$ & $5026(4)$ & $1448(10)$ & $3953(8)$ & $50(4)$ \\
\hline$C(37)$ & 5014(4) & $3298(10)$ & $3986(8)$ & $51(4)$ \\
\hline$C(38)$ & 4996(4) & $3490(10)$ & $3309(7)$ & $51(4)$ \\
\hline $\mathrm{C}(39)$ & $5366(5)$ & $2634(13)$ & $5165(8)$ & $73(7)$ \\
\hline $\mathrm{C}(40)$ & $5674(5)$ & $2307(14)$ & $5593(7)$ & $82(8)$ \\
\hline $\mathrm{C}(41)$ & $6245(5)$ & 1952(10) & $4396(10)$ & $66(6)$ \\
\hline$C(42)$ & $6486(6)$ & $2055(13)$ & $5069(11)$ & $84(7)$ \\
\hline$C(43)$ & $5920(5)$ & $2589(11)$ & $3220(9)$ & $60(5)$ \\
\hline $\mathrm{C}(44)$ & $5831(6)$ & $3302(15)$ & 2801(8) & $82(7)$ \\
\hline$C(45)$ & 6503(4) & $3289(9)$ & $4024(7)$ & $41(4)$ \\
\hline$C(46)$ & $6741(4)$ & $2814(14)$ & $3785(10)$ & $74(6)$ \\
\hline $\mathrm{C}(47)$ & 6579(6) & $10927(15)$ & $9057(10)$ & $85(7)$ \\
\hline$C(48)$ & $6635(5)$ & $10148(13)$ & $9194(9)$ & $73(6)$ \\
\hline $\mathrm{C}(49)$ & 5974(5) & $10852(12)$ & $8066(9)$ & $63(5)$ \\
\hline$C(50)$ & $5800(4)$ & $11117(13)$ & $7443(10)$ & $72(6)$ \\
\hline$C(51)$ & 6323(5) & $12242(9)$ & $8417(9)$ & $55(4)$ \\
\hline$C(52)$ & $6076(6)$ & $12416(11)$ & $8782(10)$ & $75(6)$ \\
\hline$C(53)$ & $7297(5)$ & $11688(13)$ & $7308(11)$ & $73(6)$ \\
\hline$C(54)$ & 7561(7) & $12298(17)$ & $7401(15)$ & $116(9)$ \\
\hline$C(55)$ & 6783(5) & $12741(10)$ & $7373(10)$ & $70(6)$ \\
\hline$C(56)$ & $6557(7)$ & $12667(14)$ & $6711(11)$ & $93(8)$ \\
\hline$C(57)$ & $7220(5)$ & $12036(12)$ & $8473(10)$ & $78(6)$ \\
\hline C(58) & $7424(5)$ & $11348(11)$ & $8772(10)$ & $69(5)$ \\
\hline $\mathrm{C}(63)$ & $5566(5)$ & 9793(10) & $5156(11)$ & $100(9)$ \\
\hline$C(64)$ & $6768(4)$ & $4825(9)$ & 2641(9) & $94(9)$ \\
\hline$C(65)$ & 5000 & $1060(14)$ & 2500 & $65(8)$ \\
\hline$C(66)$ & 4699(7) & $589(13)$ & $2383(10)$ & $92(8)$ \\
\hline $\mathrm{O}(67)$ & 5000 & $1730(9)$ & 2500 & $80(7)$ \\
\hline
\end{tabular}

Table 10. Bond lengths $[\AA]$ and angles $\left[^{\circ}\right]$ for BT2'.

\begin{tabular}{lr}
\hline $\operatorname{Pt}(1)-\mathrm{O}(1)$ & $2.088(9)$ \\
$\operatorname{Pt}(1)-\mathrm{N}(3)$ & $2.100(11)$
\end{tabular}




\begin{tabular}{|c|c|}
\hline $\mathrm{Pt}(1)-\mathrm{P}(2)$ & $2.247(4)$ \\
\hline $\operatorname{Pt}(1)-\mathrm{P}(1)$ & $2.249(4)$ \\
\hline $\mathrm{Pt}(2)-\mathrm{O}(3)$ & $2.093(10)$ \\
\hline $\operatorname{Pt}(2)-\mathrm{N}(4)$ & $2.101(11)$ \\
\hline $\mathrm{Pt}(2)-\mathrm{P}(4)$ & $2.240(4)$ \\
\hline $\mathrm{Pt}(2)-\mathrm{P}(3)$ & $2.263(4)$ \\
\hline $\mathrm{S}(1)-\mathrm{O}(6)$ & $1.411(11)$ \\
\hline $\mathrm{S}(1)-\mathrm{O}(5)$ & $1.429(11)$ \\
\hline $\mathrm{S}(1)-\mathrm{O}(7)$ & $1.433(10)$ \\
\hline$S(1)-C(63)$ & $1.66(3)$ \\
\hline $\mathrm{S}(2)-\mathrm{O}(10)$ & $1.388(11)$ \\
\hline $\mathrm{S}(2)-\mathrm{O}(8)$ & $1.415(10)$ \\
\hline $\mathrm{S}(2)-\mathrm{O}(9)$ & $1.477(11)$ \\
\hline$S(2)-C(64)$ & $1.77(2)$ \\
\hline $\mathrm{P}(1)-\mathrm{C}(37)$ & $1.821(18)$ \\
\hline $\mathrm{P}(1)-\mathrm{C}(35)$ & $1.823(15)$ \\
\hline P(1)-C(39) & $1.835(17)$ \\
\hline $\mathrm{P}(2)-\mathrm{C}(41)$ & $1.762(17)$ \\
\hline $\mathrm{P}(2)-\mathrm{C}(43)$ & $1.803(18)$ \\
\hline $\mathrm{P}(2)-\mathrm{C}(45)$ & $1.812(16)$ \\
\hline P(3)-C(49) & $1.775(19)$ \\
\hline $\mathrm{P}(3)-\mathrm{C}(51)$ & $1.795(16)$ \\
\hline$P(3)-C(47)$ & $1.82(2)$ \\
\hline $\mathrm{P}(4)-\mathrm{C}(57)$ & $1.79(2)$ \\
\hline $\mathrm{P}(4)-\mathrm{C}(55)$ & $1.83(2)$ \\
\hline $\mathrm{P}(4)-\mathrm{C}(53)$ & $1.83(2)$ \\
\hline $\mathrm{F}(1)-\mathrm{C}(63)$ & $1.403(15)$ \\
\hline $\mathrm{F}(2)-\mathrm{C}(63)$ & $1.399(15)$ \\
\hline $\mathrm{F}(3)-\mathrm{C}(63)$ & $1.381(16)$ \\
\hline F(4)-C(64) & $1.406(14)$ \\
\hline $\mathrm{F}(5)-\mathrm{C}(64)$ & $1.383(14)$ \\
\hline $\mathrm{F}(6)-\mathrm{C}(64)$ & $1.388(13)$ \\
\hline $\mathrm{O}(1)-\mathrm{C}(21)$ & $1.31(2)$ \\
\hline $\mathrm{O}(2)-\mathrm{C}(21)$ & $1.22(2)$ \\
\hline $\mathrm{O}(3)-\mathrm{C}(34)$ & $1.336(18)$ \\
\hline $\mathrm{O}(4)-\mathrm{C}(34)$ & $1.246(19)$ \\
\hline $\mathrm{N}(1)-\mathrm{C}(1)$ & $1.363(16)$ \\
\hline $\mathrm{N}(1)-\mathrm{C}(4)$ & $1.381(15)$ \\
\hline $\mathrm{N}(1)-\mathrm{H}(1)$ & 0.88 \\
\hline $\mathrm{N}(2)-\mathrm{C}(9)$ & $1.381(15)$ \\
\hline $\mathrm{N}(2)-\mathrm{C}(6)$ & $1.392(16)$ \\
\hline $\mathrm{N}(3)-\mathrm{C}(14)$ & $1.350(17)$ \\
\hline $\mathrm{N}(3)-\mathrm{C}(13)$ & $1.362(17)$ \\
\hline
\end{tabular}




\begin{tabular}{|c|c|}
\hline $\mathrm{N}(4)-\mathrm{C}(19)$ & $1.338(17)$ \\
\hline N(4)-C(18) & $1.345(17)$ \\
\hline$C(1)-C(2)$ & $1.421(18)$ \\
\hline$C(1)-C(10) \# 1$ & $1.421(17)$ \\
\hline$C(2)-C(3)$ & $1.36(2)$ \\
\hline $\mathrm{C}(2)-\mathrm{H}(2)$ & 0.95 \\
\hline$C(3)-C(4)$ & $1.464(18)$ \\
\hline $\mathrm{C}(3)-\mathrm{H}(3)$ & 0.95 \\
\hline$C(4)-C(5)$ & $1.394(18)$ \\
\hline$C(5)-C(6)$ & $1.376(18)$ \\
\hline$C(5)-C(11)$ & $1.474(17)$ \\
\hline $\mathrm{C}(6)-\mathrm{C}(7)$ & $1.444(17)$ \\
\hline$C(7)-C(8)$ & $1.346(19)$ \\
\hline $\mathrm{C}(7)-\mathrm{H}(7)$ & 0.95 \\
\hline$C(8)-C(9)$ & $1.454(18)$ \\
\hline $\mathrm{C}(8)-\mathrm{H}(8)$ & 0.95 \\
\hline $\mathrm{C}(9)-\mathrm{C}(10)$ & $1.387(18)$ \\
\hline$C(10)-C(16)$ & $1.479(17)$ \\
\hline$C(11)-C(15)$ & $1.392(19)$ \\
\hline $\mathrm{C}(11)-\mathrm{C}(12)$ & $1.394(19)$ \\
\hline$C(12)-C(13)$ & $1.368(18)$ \\
\hline $\mathrm{C}(12)-\mathrm{H}(12)$ & 0.95 \\
\hline $\mathrm{C}(13)-\mathrm{H}(13)$ & 0.95 \\
\hline$C(14)-C(15)$ & $1.361(18)$ \\
\hline $\mathrm{C}(14)-\mathrm{H}(14)$ & 0.95 \\
\hline $\mathrm{C}(15)-\mathrm{H}(15)$ & 0.95 \\
\hline$C(16)-C(20)$ & $1.379(17)$ \\
\hline$C(16)-C(17)$ & $1.389(17)$ \\
\hline $\mathrm{C}(17)-\mathrm{C}(18)$ & $1.352(18)$ \\
\hline $\mathrm{C}(17)-\mathrm{H}(17)$ & 0.95 \\
\hline $\mathrm{C}(18)-\mathrm{H}(18)$ & 0.95 \\
\hline$C(19)-C(20)$ & $1.362(18)$ \\
\hline $\mathrm{C}(19)-\mathrm{H}(19)$ & 0.95 \\
\hline $\mathrm{C}(20)-\mathrm{H}(20)$ & 0.95 \\
\hline$C(21)-C(22)$ & $1.50(2)$ \\
\hline$C(22)-C(27)$ & $1.39(2)$ \\
\hline$C(22)-C(23)$ & $1.41(2)$ \\
\hline$C(23)-C(24)$ & $1.39(2)$ \\
\hline $\mathrm{C}(23)-\mathrm{H}(23)$ & 0.95 \\
\hline$C(24)-C(25)$ & $1.40(2)$ \\
\hline $\mathrm{C}(24)-\mathrm{H}(24)$ & 0.95 \\
\hline$C(25)-C(26)$ & $1.36(2)$ \\
\hline$C(25)-C(28)$ & $1.465(19)$ \\
\hline
\end{tabular}




\begin{tabular}{|c|c|}
\hline$C(26)-C(27)$ & $1.40(2)$ \\
\hline $\mathrm{C}(26)-\mathrm{H}(26)$ & 0.95 \\
\hline C(27)-H(27) & 0.95 \\
\hline C(28)-C(33) & $1.36(2)$ \\
\hline C(28)-C(29) & $1.41(2)$ \\
\hline C(29)-C(30) & $1.39(2)$ \\
\hline C(29)-H(29) & 0.95 \\
\hline $\mathrm{C}(30)-\mathrm{C}(31)$ & $1.32(2)$ \\
\hline C(30)-H(30) & 0.95 \\
\hline $\mathrm{C}(31)-\mathrm{C}(32)$ & $1.37(2)$ \\
\hline C(31)-C(34) & $1.53(2)$ \\
\hline C(32)-C(33) & $1.37(2)$ \\
\hline $\mathrm{C}(32)-\mathrm{H}(32)$ & 0.95 \\
\hline C(33)-H(33) & 0.95 \\
\hline C(35)-C(36) & $1.541(19)$ \\
\hline $\mathrm{C}(35)-\mathrm{H}(35 \mathrm{~A})$ & 0.99 \\
\hline $\mathrm{C}(35)-\mathrm{H}(35 \mathrm{~B})$ & 0.99 \\
\hline $\mathrm{C}(36)-\mathrm{H}(36 \mathrm{~A})$ & 0.98 \\
\hline $\mathrm{C}(36)-\mathrm{H}(36 \mathrm{~B})$ & 0.98 \\
\hline $\mathrm{C}(36)-\mathrm{H}(36 \mathrm{C})$ & 0.98 \\
\hline $\mathrm{C}(37)-\mathrm{C}(38)$ & $1.56(2)$ \\
\hline $\mathrm{C}(37)-\mathrm{H}(37 \mathrm{~A})$ & 0.99 \\
\hline $\mathrm{C}(37)-\mathrm{H}(37 \mathrm{~B})$ & 0.99 \\
\hline $\mathrm{C}(38)-\mathrm{H}(38 \mathrm{~A})$ & 0.98 \\
\hline $\mathrm{C}(38)-\mathrm{H}(38 \mathrm{~B})$ & 0.98 \\
\hline $\mathrm{C}(38)-\mathrm{H}(38 \mathrm{C})$ & 0.98 \\
\hline C(39)-C(40) & $1.50(3)$ \\
\hline C(39)-H(39A) & 0.99 \\
\hline C(39)-H(39B) & 0.99 \\
\hline $\mathrm{C}(40)-\mathrm{H}(40 \mathrm{~A})$ & 0.98 \\
\hline $\mathrm{C}(40)-\mathrm{H}(40 \mathrm{~B})$ & 0.98 \\
\hline $\mathrm{C}(40)-\mathrm{H}(40 \mathrm{C})$ & 0.98 \\
\hline$C(41)-C(42)$ & $1.58(3)$ \\
\hline $\mathrm{C}(41)-\mathrm{H}(41 \mathrm{~A})$ & 0.99 \\
\hline $\mathrm{C}(41)-\mathrm{H}(41 \mathrm{~B})$ & 0.99 \\
\hline $\mathrm{C}(42)-\mathrm{H}(42 \mathrm{~A})$ & 0.98 \\
\hline $\mathrm{C}(42)-\mathrm{H}(42 \mathrm{~B})$ & 0.98 \\
\hline $\mathrm{C}(42)-\mathrm{H}(42 \mathrm{C})$ & 0.98 \\
\hline$C(43)-C(44)$ & $1.55(3)$ \\
\hline $\mathrm{C}(43)-\mathrm{H}(43 \mathrm{~A})$ & 0.99 \\
\hline $\mathrm{C}(43)-\mathrm{H}(43 \mathrm{~B})$ & 0.99 \\
\hline $\mathrm{C}(44)-\mathrm{H}(44 \mathrm{~A})$ & 0.98 \\
\hline $\mathrm{C}(44)-\mathrm{H}(44 \mathrm{~B})$ & 0.98 \\
\hline
\end{tabular}




\begin{tabular}{|c|c|}
\hline $\mathrm{C}(44)-\mathrm{H}(44 \mathrm{C})$ & 0.98 \\
\hline$C(45)-C(46)$ & $1.53(2)$ \\
\hline $\mathrm{C}(45)-\mathrm{H}(45 \mathrm{~A})$ & 0.99 \\
\hline $\mathrm{C}(45)-\mathrm{H}(45 \mathrm{~B})$ & 0.99 \\
\hline $\mathrm{C}(46)-\mathrm{H}(46 \mathrm{~A})$ & 0.98 \\
\hline $\mathrm{C}(46)-\mathrm{H}(46 \mathrm{~B})$ & 0.98 \\
\hline $\mathrm{C}(46)-\mathrm{H}(46 \mathrm{C})$ & 0.98 \\
\hline$C(47)-C(48)$ & $1.41(3)$ \\
\hline $\mathrm{C}(47)-\mathrm{H}(47 \mathrm{~A})$ & 0.99 \\
\hline $\mathrm{C}(47)-\mathrm{H}(47 \mathrm{~B})$ & 0.99 \\
\hline $\mathrm{C}(48)-\mathrm{H}(48 \mathrm{~A})$ & 0.98 \\
\hline $\mathrm{C}(48)-\mathrm{H}(48 \mathrm{~B})$ & 0.98 \\
\hline $\mathrm{C}(48)-\mathrm{H}(48 \mathrm{C})$ & 0.98 \\
\hline C(49)-C(50) & $1.47(3)$ \\
\hline $\mathrm{C}(49)-\mathrm{H}(49 \mathrm{~A})$ & 0.99 \\
\hline $\mathrm{C}(49)-\mathrm{H}(49 \mathrm{~B})$ & 0.99 \\
\hline $\mathrm{C}(50)-\mathrm{H}(50 \mathrm{~A})$ & 0.98 \\
\hline $\mathrm{C}(50)-\mathrm{H}(50 \mathrm{~B})$ & 0.98 \\
\hline $\mathrm{C}(50)-\mathrm{H}(50 \mathrm{C})$ & 0.98 \\
\hline$C(51)-C(52)$ & $1.55(3)$ \\
\hline $\mathrm{C}(51)-\mathrm{H}(51 \mathrm{~A})$ & 0.99 \\
\hline $\mathrm{C}(51)-\mathrm{H}(51 \mathrm{~B})$ & 0.99 \\
\hline $\mathrm{C}(52)-\mathrm{H}(52 \mathrm{~A})$ & 0.98 \\
\hline $\mathrm{C}(52)-\mathrm{H}(52 \mathrm{~B})$ & 0.98 \\
\hline $\mathrm{C}(52)-\mathrm{H}(52 \mathrm{C})$ & 0.98 \\
\hline$C(53)-C(54)$ & $1.52(3)$ \\
\hline $\mathrm{C}(53)-\mathrm{H}(53 \mathrm{~A})$ & 0.99 \\
\hline $\mathrm{C}(53)-\mathrm{H}(53 \mathrm{~B})$ & 0.99 \\
\hline $\mathrm{C}(54)-\mathrm{H}(54 \mathrm{~A})$ & 0.98 \\
\hline $\mathrm{C}(54)-\mathrm{H}(54 \mathrm{~B})$ & 0.98 \\
\hline $\mathrm{C}(54)-\mathrm{H}(54 \mathrm{C})$ & 0.98 \\
\hline C(55)-C(56) & $1.53(3)$ \\
\hline $\mathrm{C}(55)-\mathrm{H}(55 \mathrm{~A})$ & 0.99 \\
\hline $\mathrm{C}(55)-\mathrm{H}(55 \mathrm{~B})$ & 0.99 \\
\hline $\mathrm{C}(56)-\mathrm{H}(56 \mathrm{~A})$ & 0.98 \\
\hline $\mathrm{C}(56)-\mathrm{H}(56 \mathrm{~B})$ & 0.98 \\
\hline $\mathrm{C}(56)-\mathrm{H}(56 \mathrm{C})$ & 0.98 \\
\hline C(57)-C(58) & $1.52(3)$ \\
\hline $\mathrm{C}(57)-\mathrm{H}(57 \mathrm{~A})$ & 0.99 \\
\hline $\mathrm{C}(57)-\mathrm{H}(57 \mathrm{~B})$ & 0.99 \\
\hline $\mathrm{C}(58)-\mathrm{H}(58 \mathrm{~A})$ & 0.98 \\
\hline $\mathrm{C}(58)-\mathrm{H}(58 \mathrm{~B})$ & 0.98 \\
\hline $\mathrm{C}(58)-\mathrm{H}(58 \mathrm{C})$ & 0.98 \\
\hline
\end{tabular}




\begin{tabular}{|c|c|}
\hline $\mathrm{C}(65)-\mathrm{O}(67)$ & $1.17(3)$ \\
\hline $\mathrm{C}(65)-\mathrm{C}(66) \# 2$ & $1.48(3)$ \\
\hline$C(65)-C(66)$ & $1.48(3)$ \\
\hline $\mathrm{C}(66)-\mathrm{H}(66 \mathrm{~A})$ & 0.98 \\
\hline $\mathrm{C}(66)-\mathrm{H}(66 \mathrm{~B})$ & 0.98 \\
\hline $\mathrm{C}(66)-\mathrm{H}(66 \mathrm{C})$ & 0.98 \\
\hline $\mathrm{O}(1)-\mathrm{Pt}(1)-\mathrm{N}(3)$ & $82.7(4)$ \\
\hline $\mathrm{O}(1)-\mathrm{Pt}(1)-\mathrm{P}(2)$ & $172.6(3)$ \\
\hline $\mathrm{N}(3)-\operatorname{Pt}(1)-\mathrm{P}(2)$ & $92.9(3)$ \\
\hline $\mathrm{O}(1)-\mathrm{Pt}(1)-\mathrm{P}(1)$ & $84.5(3)$ \\
\hline $\mathrm{N}(3)-\operatorname{Pt}(1)-\mathrm{P}(1)$ & $166.9(3)$ \\
\hline $\mathrm{P}(2)-\mathrm{Pt}(1)-\mathrm{P}(1)$ & $100.16(14)$ \\
\hline $\mathrm{O}(3)-\mathrm{Pt}(2)-\mathrm{N}(4)$ & $82.2(4)$ \\
\hline $\mathrm{O}(3)-\mathrm{Pt}(2)-\mathrm{P}(4)$ & $175.6(3)$ \\
\hline $\mathrm{N}(4)-\mathrm{Pt}(2)-\mathrm{P}(4)$ & $93.7(3)$ \\
\hline $\mathrm{O}(3)-\mathrm{Pt}(2)-\mathrm{P}(3)$ & $86.0(3)$ \\
\hline $\mathrm{N}(4)-\operatorname{Pt}(2)-\mathrm{P}(3)$ & $168.2(3)$ \\
\hline $\mathrm{P}(4)-\mathrm{Pt}(2)-\mathrm{P}(3)$ & $98.10(15)$ \\
\hline $\mathrm{O}(6)-\mathrm{S}(1)-\mathrm{O}(5)$ & $115.9(11)$ \\
\hline $\mathrm{O}(6)-\mathrm{S}(1)-\mathrm{O}(7)$ & $116.5(10)$ \\
\hline $\mathrm{O}(5)-\mathrm{S}(1)-\mathrm{O}(7)$ & $113.5(9)$ \\
\hline $\mathrm{O}(6)-\mathrm{S}(1)-\mathrm{C}(63)$ & $104.8(11)$ \\
\hline $\mathrm{O}(5)-\mathrm{S}(1)-\mathrm{C}(63)$ & $100.5(11)$ \\
\hline $\mathrm{O}(7)-\mathrm{S}(1)-\mathrm{C}(63)$ & $102.7(9)$ \\
\hline $\mathrm{O}(10)-\mathrm{S}(2)-\mathrm{O}(8)$ & $122.1(10)$ \\
\hline $\mathrm{O}(10)-\mathrm{S}(2)-\mathrm{O}(9)$ & $110.9(10)$ \\
\hline $\mathrm{O}(8)-\mathrm{S}(2)-\mathrm{O}(9)$ & $111.7(9)$ \\
\hline $\mathrm{O}(10)-\mathrm{S}(2)-\mathrm{C}(64)$ & $105.0(8)$ \\
\hline $\mathrm{O}(8)-\mathrm{S}(2)-\mathrm{C}(64)$ & $101.9(8)$ \\
\hline $\mathrm{O}(9)-\mathrm{S}(2)-\mathrm{C}(64)$ & $102.7(8)$ \\
\hline $\mathrm{C}(37)-\mathrm{P}(1)-\mathrm{C}(35)$ & $107.2(8)$ \\
\hline $\mathrm{C}(37)-\mathrm{P}(1)-\mathrm{C}(39)$ & $105.2(10)$ \\
\hline $\mathrm{C}(35)-\mathrm{P}(1)-\mathrm{C}(39)$ & $105.0(9)$ \\
\hline $\mathrm{C}(37)-\mathrm{P}(1)-\mathrm{Pt}(1)$ & $107.3(6)$ \\
\hline $\mathrm{C}(35)-\mathrm{P}(1)-\mathrm{Pt}(1)$ & $122.0(5)$ \\
\hline $\mathrm{C}(39)-\mathrm{P}(1)-\mathrm{Pt}(1)$ & 109.0(6) \\
\hline $\mathrm{C}(41)-\mathrm{P}(2)-\mathrm{C}(43)$ & $106.3(10)$ \\
\hline $\mathrm{C}(41)-\mathrm{P}(2)-\mathrm{C}(45)$ & $104.4(8)$ \\
\hline $\mathrm{C}(43)-\mathrm{P}(2)-\mathrm{C}(45)$ & $105.6(8)$ \\
\hline $\mathrm{C}(41)-\mathrm{P}(2)-\mathrm{Pt}(1)$ & $115.2(7)$ \\
\hline $\mathrm{C}(43)-\mathrm{P}(2)-\operatorname{Pt}(1)$ & $113.6(7)$ \\
\hline $\mathrm{C}(45)-\mathrm{P}(2)-\mathrm{Pt}(1)$ & $110.9(5)$ \\
\hline
\end{tabular}




\begin{tabular}{|c|c|}
\hline $\mathrm{C}(49)-\mathrm{P}(3)-\mathrm{C}(51)$ & $105.5(9)$ \\
\hline $\mathrm{C}(49)-\mathrm{P}(3)-\mathrm{C}(47)$ & $106.5(11)$ \\
\hline $\mathrm{C}(51)-\mathrm{P}(3)-\mathrm{C}(47)$ & $100.3(11)$ \\
\hline C(49)-P(3)-Pt(2) & $109.6(6)$ \\
\hline $\mathrm{C}(51)-\mathrm{P}(3)-\mathrm{Pt}(2)$ & $122.4(7)$ \\
\hline C(47)-P(3)-Pt(2) & $111.2(7)$ \\
\hline C(57)-P(4)-C(55) & $107.3(10)$ \\
\hline C(57)-P(4)-C(53) & $103.4(10)$ \\
\hline C(55)-P(4)-C(53) & $104.6(10)$ \\
\hline C(57)-P(4)-Pt(2) & $112.1(8)$ \\
\hline C(55)-P(4)-Pt(2) & $115.8(7)$ \\
\hline C(53)-P(4)-Pt(2) & $112.5(7)$ \\
\hline $\mathrm{C}(21)-\mathrm{O}(1)-\mathrm{Pt}(1)$ & $115.7(10)$ \\
\hline $\mathrm{C}(34)-\mathrm{O}(3)-\mathrm{Pt}(2)$ & $114.3(10)$ \\
\hline C(1)-N(1)-C(4) & $105.8(10)$ \\
\hline $\mathrm{C}(1)-\mathrm{N}(1)-\mathrm{H}(1)$ & 127.1 \\
\hline $\mathrm{C}(4)-\mathrm{N}(1)-\mathrm{H}(1)$ & 127.1 \\
\hline $\mathrm{C}(9)-\mathrm{N}(2)-\mathrm{C}(6)$ & $109.9(10)$ \\
\hline C(14)-N(3)-C(13) & $118.4(11)$ \\
\hline C(14)-N(3)-Pt(1) & 119.1(9) \\
\hline C(13)-N(3)-Pt(1) & $122.2(8)$ \\
\hline C(19)-N(4)-C(18) & $115.7(11)$ \\
\hline C(19)-N(4)-Pt(2) & $122.5(8)$ \\
\hline C(18)-N(4)-Pt(2) & $120.1(9)$ \\
\hline $\mathrm{N}(1)-\mathrm{C}(1)-\mathrm{C}(2)$ & $111.5(11)$ \\
\hline$N(1)-C(1)-C(10) \# 1$ & $125.6(11)$ \\
\hline $\mathrm{C}(2)-\mathrm{C}(1)-\mathrm{C}(10) \# 1$ & $122.8(12)$ \\
\hline $\mathrm{C}(3)-\mathrm{C}(2)-\mathrm{C}(1)$ & $107.0(12)$ \\
\hline $\mathrm{C}(3)-\mathrm{C}(2)-\mathrm{H}(2)$ & 126.5 \\
\hline $\mathrm{C}(1)-\mathrm{C}(2)-\mathrm{H}(2)$ & 126.5 \\
\hline$C(2)-C(3)-C(4)$ & $106.5(12)$ \\
\hline $\mathrm{C}(2)-\mathrm{C}(3)-\mathrm{H}(3)$ & 126.7 \\
\hline $\mathrm{C}(4)-\mathrm{C}(3)-\mathrm{H}(3)$ & 126.7 \\
\hline $\mathrm{N}(1)-\mathrm{C}(4)-\mathrm{C}(5)$ & $127.6(11)$ \\
\hline $\mathrm{N}(1)-\mathrm{C}(4)-\mathrm{C}(3)$ & $109.0(11)$ \\
\hline$C(5)-C(4)-C(3)$ & $123.3(11)$ \\
\hline$C(6)-C(5)-C(4)$ & $127.0(11)$ \\
\hline$C(6)-C(5)-C(11)$ & $118.2(12)$ \\
\hline $\mathrm{C}(4)-\mathrm{C}(5)-\mathrm{C}(11)$ & $114.8(11)$ \\
\hline $\mathrm{C}(5)-\mathrm{C}(6)-\mathrm{N}(2)$ & $125.3(11)$ \\
\hline$C(5)-C(6)-C(7)$ & $128.4(12)$ \\
\hline $\mathrm{N}(2)-\mathrm{C}(6)-\mathrm{C}(7)$ & $106.3(11)$ \\
\hline$C(8)-C(7)-C(6)$ & $108.8(11)$ \\
\hline
\end{tabular}




\begin{tabular}{|c|c|}
\hline $\mathrm{C}(8)-\mathrm{C}(7)-\mathrm{H}(7)$ & 125.6 \\
\hline $\mathrm{C}(6)-\mathrm{C}(7)-\mathrm{H}(7)$ & 125.6 \\
\hline $\mathrm{C}(7)-\mathrm{C}(8)-\mathrm{C}(9)$ & $108.3(11)$ \\
\hline $\mathrm{C}(7)-\mathrm{C}(8)-\mathrm{H}(8)$ & 125.8 \\
\hline $\mathrm{C}(9)-\mathrm{C}(8)-\mathrm{H}(8)$ & 125.8 \\
\hline $\mathrm{N}(2)-\mathrm{C}(9)-\mathrm{C}(10)$ & $126.6(11)$ \\
\hline $\mathrm{N}(2)-\mathrm{C}(9)-\mathrm{C}(8)$ & $106.5(11)$ \\
\hline $\mathrm{C}(10)-\mathrm{C}(9)-\mathrm{C}(8)$ & $126.8(11)$ \\
\hline $\mathrm{C}(9)-\mathrm{C}(10)-\mathrm{C}(1) \# 1$ & $123.7(11)$ \\
\hline$C(9)-C(10)-C(16)$ & $120.2(11)$ \\
\hline $\mathrm{C}(1) \# 1-\mathrm{C}(10)-\mathrm{C}(16)$ & $115.9(11)$ \\
\hline$C(15)-C(11)-C(12)$ & $117.1(12)$ \\
\hline $\mathrm{C}(15)-\mathrm{C}(11)-\mathrm{C}(5)$ & $121.9(12)$ \\
\hline$C(12)-C(11)-C(5)$ & $120.8(12)$ \\
\hline$C(13)-C(12)-C(11)$ & $120.3(12)$ \\
\hline $\mathrm{C}(13)-\mathrm{C}(12)-\mathrm{H}(12)$ & 119.9 \\
\hline $\mathrm{C}(11)-\mathrm{C}(12)-\mathrm{H}(12)$ & 119.9 \\
\hline $\mathrm{N}(3)-\mathrm{C}(13)-\mathrm{C}(12)$ & $121.5(12)$ \\
\hline N(3)-C(13)-H(13) & 119.3 \\
\hline $\mathrm{C}(12)-\mathrm{C}(13)-\mathrm{H}(13)$ & 119.3 \\
\hline $\mathrm{N}(3)-\mathrm{C}(14)-\mathrm{C}(15)$ & $121.8(13)$ \\
\hline $\mathrm{N}(3)-\mathrm{C}(14)-\mathrm{H}(14)$ & 119.1 \\
\hline $\mathrm{C}(15)-\mathrm{C}(14)-\mathrm{H}(14)$ & 119.1 \\
\hline$C(14)-C(15)-C(11)$ & $120.7(13)$ \\
\hline $\mathrm{C}(14)-\mathrm{C}(15)-\mathrm{H}(15)$ & 119.7 \\
\hline $\mathrm{C}(11)-\mathrm{C}(15)-\mathrm{H}(15)$ & 119.7 \\
\hline$C(20)-C(16)-C(17)$ & $117.3(12)$ \\
\hline$C(20)-C(16)-C(10)$ & $118.4(11)$ \\
\hline$C(17)-C(16)-C(10)$ & $124.2(11)$ \\
\hline$C(18)-C(17)-C(16)$ & $119.1(12)$ \\
\hline $\mathrm{C}(18)-\mathrm{C}(17)-\mathrm{H}(17)$ & 120.4 \\
\hline $\mathrm{C}(16)-\mathrm{C}(17)-\mathrm{H}(17)$ & 120.4 \\
\hline $\mathrm{N}(4)-\mathrm{C}(18)-\mathrm{C}(17)$ & $124.4(13)$ \\
\hline $\mathrm{N}(4)-\mathrm{C}(18)-\mathrm{H}(18)$ & 117.8 \\
\hline $\mathrm{C}(17)-\mathrm{C}(18)-\mathrm{H}(18)$ & 117.8 \\
\hline N(4)-C(19)-C(20) & $123.7(12)$ \\
\hline $\mathrm{N}(4)-\mathrm{C}(19)-\mathrm{H}(19)$ & 118.1 \\
\hline $\mathrm{C}(20)-\mathrm{C}(19)-\mathrm{H}(19)$ & 118.1 \\
\hline$C(19)-C(20)-C(16)$ & $119.6(12)$ \\
\hline $\mathrm{C}(19)-\mathrm{C}(20)-\mathrm{H}(20)$ & 120.2 \\
\hline $\mathrm{C}(16)-\mathrm{C}(20)-\mathrm{H}(20)$ & 120.2 \\
\hline $\mathrm{O}(2)-\mathrm{C}(21)-\mathrm{O}(1)$ & $126.2(15)$ \\
\hline $\mathrm{O}(2)-\mathrm{C}(21)-\mathrm{C}(22)$ & $119.9(17)$ \\
\hline
\end{tabular}




\begin{tabular}{|c|c|}
\hline $\mathrm{O}(1)-\mathrm{C}(21)-\mathrm{C}(22)$ & $113.8(15)$ \\
\hline$C(27)-C(22)-C(23)$ & $118.6(14)$ \\
\hline$C(27)-C(22)-C(21)$ & $121.1(15)$ \\
\hline $\mathrm{C}(23)-\mathrm{C}(22)-\mathrm{C}(21)$ & $119.9(14)$ \\
\hline$C(24)-C(23)-C(22)$ & $120.1(15)$ \\
\hline $\mathrm{C}(24)-\mathrm{C}(23)-\mathrm{H}(23)$ & 119.9 \\
\hline $\mathrm{C}(22)-\mathrm{C}(23)-\mathrm{H}(23)$ & 119.9 \\
\hline $\mathrm{C}(23)-\mathrm{C}(24)-\mathrm{C}(25)$ & $120.3(17)$ \\
\hline $\mathrm{C}(23)-\mathrm{C}(24)-\mathrm{H}(24)$ & 119.9 \\
\hline $\mathrm{C}(25)-\mathrm{C}(24)-\mathrm{H}(24)$ & 119.9 \\
\hline$C(26)-C(25)-C(24)$ & $119.3(15)$ \\
\hline $\mathrm{C}(26)-\mathrm{C}(25)-\mathrm{C}(28)$ & $122.6(14)$ \\
\hline$C(24)-C(25)-C(28)$ & $118.0(14)$ \\
\hline$C(25)-C(26)-C(27)$ & $121.3(15)$ \\
\hline $\mathrm{C}(25)-\mathrm{C}(26)-\mathrm{H}(26)$ & 119.4 \\
\hline $\mathrm{C}(27)-\mathrm{C}(26)-\mathrm{H}(26)$ & 119.4 \\
\hline$C(22)-C(27)-C(26)$ & $120.3(14)$ \\
\hline $\mathrm{C}(22)-\mathrm{C}(27)-\mathrm{H}(27)$ & 119.9 \\
\hline $\mathrm{C}(26)-\mathrm{C}(27)-\mathrm{H}(27)$ & 119.9 \\
\hline$C(33)-C(28)-C(29)$ & $115.3(13)$ \\
\hline$C(33)-C(28)-C(25)$ & $122.2(14)$ \\
\hline $\mathrm{C}(29)-\mathrm{C}(28)-\mathrm{C}(25)$ & $122.4(12)$ \\
\hline$C(30)-C(29)-C(28)$ & $121.2(13)$ \\
\hline $\mathrm{C}(30)-\mathrm{C}(29)-\mathrm{H}(29)$ & 119.4 \\
\hline $\mathrm{C}(28)-\mathrm{C}(29)-\mathrm{H}(29)$ & 119.4 \\
\hline$C(31)-C(30)-C(29)$ & $120.8(15)$ \\
\hline $\mathrm{C}(31)-\mathrm{C}(30)-\mathrm{H}(30)$ & 119.6 \\
\hline $\mathrm{C}(29)-\mathrm{C}(30)-\mathrm{H}(30)$ & 119.6 \\
\hline$C(30)-C(31)-C(32)$ & $119.6(14)$ \\
\hline $\mathrm{C}(30)-\mathrm{C}(31)-\mathrm{C}(34)$ & $119.3(14)$ \\
\hline $\mathrm{C}(32)-\mathrm{C}(31)-\mathrm{C}(34)$ & $121.2(13)$ \\
\hline $\mathrm{C}(33)-\mathrm{C}(32)-\mathrm{C}(31)$ & $120.2(14)$ \\
\hline $\mathrm{C}(33)-\mathrm{C}(32)-\mathrm{H}(32)$ & 119.9 \\
\hline $\mathrm{C}(31)-\mathrm{C}(32)-\mathrm{H}(32)$ & 119.9 \\
\hline $\mathrm{C}(28)-\mathrm{C}(33)-\mathrm{C}(32)$ & $122.8(14)$ \\
\hline $\mathrm{C}(28)-\mathrm{C}(33)-\mathrm{H}(33)$ & 118.6 \\
\hline $\mathrm{C}(32)-\mathrm{C}(33)-\mathrm{H}(33)$ & 118.6 \\
\hline $\mathrm{O}(4)-\mathrm{C}(34)-\mathrm{O}(3)$ & $127.3(14)$ \\
\hline $\mathrm{O}(4)-\mathrm{C}(34)-\mathrm{C}(31)$ & $120.1(13)$ \\
\hline $\mathrm{O}(3)-\mathrm{C}(34)-\mathrm{C}(31)$ & $112.1(14)$ \\
\hline $\mathrm{C}(36)-\mathrm{C}(35)-\mathrm{P}(1)$ & $114.2(11)$ \\
\hline $\mathrm{C}(36)-\mathrm{C}(35)-\mathrm{H}(35 \mathrm{~A})$ & 108.7 \\
\hline $\mathrm{P}(1)-\mathrm{C}(35)-\mathrm{H}(35 \mathrm{~A})$ & 108.7 \\
\hline
\end{tabular}




\begin{tabular}{|c|c|}
\hline $\mathrm{C}(36)-\mathrm{C}(35)-\mathrm{H}(35 \mathrm{~B})$ & 108.7 \\
\hline $\mathrm{P}(1)-\mathrm{C}(35)-\mathrm{H}(35 \mathrm{~B})$ & 108.7 \\
\hline $\mathrm{H}(35 \mathrm{~A})-\mathrm{C}(35)-\mathrm{H}(35 \mathrm{~B})$ & 107.6 \\
\hline C(35)-C(36)-H(36A) & 109.5 \\
\hline $\mathrm{C}(35)-\mathrm{C}(36)-\mathrm{H}(36 \mathrm{~B})$ & 109.5 \\
\hline $\mathrm{H}(36 \mathrm{~A})-\mathrm{C}(36)-\mathrm{H}(36 \mathrm{~B})$ & 109.5 \\
\hline C(35)-C(36)-H(36C) & 109.5 \\
\hline $\mathrm{H}(36 \mathrm{~A})-\mathrm{C}(36)-\mathrm{H}(36 \mathrm{C})$ & 109.5 \\
\hline $\mathrm{H}(36 \mathrm{~B})-\mathrm{C}(36)-\mathrm{H}(36 \mathrm{C})$ & 109.5 \\
\hline $\mathrm{C}(38)-\mathrm{C}(37)-\mathrm{P}(1)$ & $112.2(12)$ \\
\hline $\mathrm{C}(38)-\mathrm{C}(37)-\mathrm{H}(37 \mathrm{~A})$ & 109.2 \\
\hline $\mathrm{P}(1)-\mathrm{C}(37)-\mathrm{H}(37 \mathrm{~A})$ & 109.2 \\
\hline $\mathrm{C}(38)-\mathrm{C}(37)-\mathrm{H}(37 \mathrm{~B})$ & 109.2 \\
\hline $\mathrm{P}(1)-\mathrm{C}(37)-\mathrm{H}(37 \mathrm{~B})$ & 109.2 \\
\hline $\mathrm{H}(37 \mathrm{~A})-\mathrm{C}(37)-\mathrm{H}(37 \mathrm{~B})$ & 107.9 \\
\hline C(37)-C(38)-H(38A) & 109.5 \\
\hline $\mathrm{C}(37)-\mathrm{C}(38)-\mathrm{H}(38 \mathrm{~B})$ & 109.5 \\
\hline $\mathrm{H}(38 \mathrm{~A})-\mathrm{C}(38)-\mathrm{H}(38 \mathrm{~B})$ & 109.5 \\
\hline $\mathrm{C}(37)-\mathrm{C}(38)-\mathrm{H}(38 \mathrm{C})$ & 109.5 \\
\hline $\mathrm{H}(38 \mathrm{~A})-\mathrm{C}(38)-\mathrm{H}(38 \mathrm{C})$ & 109.5 \\
\hline $\mathrm{H}(38 \mathrm{~B})-\mathrm{C}(38)-\mathrm{H}(38 \mathrm{C})$ & 109.5 \\
\hline $\mathrm{C}(40)-\mathrm{C}(39)-\mathrm{P}(1)$ & $113.5(14)$ \\
\hline $\mathrm{C}(40)-\mathrm{C}(39)-\mathrm{H}(39 \mathrm{~A})$ & 108.9 \\
\hline $\mathrm{P}(1)-\mathrm{C}(39)-\mathrm{H}(39 \mathrm{~A})$ & 108.9 \\
\hline $\mathrm{C}(40)-\mathrm{C}(39)-\mathrm{H}(39 \mathrm{~B})$ & 108.9 \\
\hline $\mathrm{P}(1)-\mathrm{C}(39)-\mathrm{H}(39 \mathrm{~B})$ & 108.9 \\
\hline $\mathrm{H}(39 \mathrm{~A})-\mathrm{C}(39)-\mathrm{H}(39 \mathrm{~B})$ & 107.7 \\
\hline $\mathrm{C}(39)-\mathrm{C}(40)-\mathrm{H}(40 \mathrm{~A})$ & 109.5 \\
\hline C(39)-C(40)-H(40B) & 109.5 \\
\hline $\mathrm{H}(40 \mathrm{~A})-\mathrm{C}(40)-\mathrm{H}(40 \mathrm{~B})$ & 109.5 \\
\hline C(39)-C(40)-H(40C) & 109.5 \\
\hline $\mathrm{H}(40 \mathrm{~A})-\mathrm{C}(40)-\mathrm{H}(40 \mathrm{C})$ & 109.5 \\
\hline $\mathrm{H}(40 \mathrm{~B})-\mathrm{C}(40)-\mathrm{H}(40 \mathrm{C})$ & 109.5 \\
\hline$C(42)-C(41)-P(2)$ & $113.2(15)$ \\
\hline $\mathrm{C}(42)-\mathrm{C}(41)-\mathrm{H}(41 \mathrm{~A})$ & 108.9 \\
\hline $\mathrm{P}(2)-\mathrm{C}(41)-\mathrm{H}(41 \mathrm{~A})$ & 108.9 \\
\hline $\mathrm{C}(42)-\mathrm{C}(41)-\mathrm{H}(41 \mathrm{~B})$ & 108.9 \\
\hline $\mathrm{P}(2)-\mathrm{C}(41)-\mathrm{H}(41 \mathrm{~B})$ & 108.9 \\
\hline $\mathrm{H}(41 \mathrm{~A})-\mathrm{C}(41)-\mathrm{H}(41 \mathrm{~B})$ & 107.8 \\
\hline $\mathrm{C}(41)-\mathrm{C}(42)-\mathrm{H}(42 \mathrm{~A})$ & 109.5 \\
\hline $\mathrm{C}(41)-\mathrm{C}(42)-\mathrm{H}(42 \mathrm{~B})$ & 109.5 \\
\hline $\mathrm{H}(42 \mathrm{~A})-\mathrm{C}(42)-\mathrm{H}(42 \mathrm{~B})$ & 109.5 \\
\hline $\mathrm{C}(41)-\mathrm{C}(42)-\mathrm{H}(42 \mathrm{C})$ & 109.5 \\
\hline
\end{tabular}




\begin{tabular}{|c|c|}
\hline $\mathrm{H}(42 \mathrm{~A})-\mathrm{C}(42)-\mathrm{H}(42 \mathrm{C})$ & 109.5 \\
\hline $\mathrm{H}(42 \mathrm{~B})-\mathrm{C}(42)-\mathrm{H}(42 \mathrm{C})$ & 109.5 \\
\hline C(44)-C(43)-P(2) & $113.0(13)$ \\
\hline $\mathrm{C}(44)-\mathrm{C}(43)-\mathrm{H}(43 \mathrm{~A})$ & 109 \\
\hline $\mathrm{P}(2)-\mathrm{C}(43)-\mathrm{H}(43 \mathrm{~A})$ & 109 \\
\hline $\mathrm{C}(44)-\mathrm{C}(43)-\mathrm{H}(43 \mathrm{~B})$ & 109 \\
\hline $\mathrm{P}(2)-\mathrm{C}(43)-\mathrm{H}(43 \mathrm{~B})$ & 109 \\
\hline $\mathrm{H}(43 \mathrm{~A})-\mathrm{C}(43)-\mathrm{H}(43 \mathrm{~B})$ & 107.8 \\
\hline C(43)-C(44)-H(44A) & 109.5 \\
\hline $\mathrm{C}(43)-\mathrm{C}(44)-\mathrm{H}(44 \mathrm{~B})$ & 109.5 \\
\hline $\mathrm{H}(44 \mathrm{~A})-\mathrm{C}(44)-\mathrm{H}(44 \mathrm{~B})$ & 109.5 \\
\hline C(43)-C(44)-H(44C) & 109.5 \\
\hline $\mathrm{H}(44 \mathrm{~A})-\mathrm{C}(44)-\mathrm{H}(44 \mathrm{C})$ & 109.5 \\
\hline $\mathrm{H}(44 \mathrm{~B})-\mathrm{C}(44)-\mathrm{H}(44 \mathrm{C})$ & 109.5 \\
\hline $\mathrm{C}(46)-\mathrm{C}(45)-\mathrm{P}(2)$ & $115.9(12)$ \\
\hline $\mathrm{C}(46)-\mathrm{C}(45)-\mathrm{H}(45 \mathrm{~A})$ & 108.3 \\
\hline $\mathrm{P}(2)-\mathrm{C}(45)-\mathrm{H}(45 \mathrm{~A})$ & 108.3 \\
\hline $\mathrm{C}(46)-\mathrm{C}(45)-\mathrm{H}(45 \mathrm{~B})$ & 108.3 \\
\hline $\mathrm{P}(2)-\mathrm{C}(45)-\mathrm{H}(45 \mathrm{~B})$ & 108.3 \\
\hline $\mathrm{H}(45 \mathrm{~A})-\mathrm{C}(45)-\mathrm{H}(45 \mathrm{~B})$ & 107.4 \\
\hline $\mathrm{C}(45)-\mathrm{C}(46)-\mathrm{H}(46 \mathrm{~A})$ & 109.5 \\
\hline $\mathrm{C}(45)-\mathrm{C}(46)-\mathrm{H}(46 \mathrm{~B})$ & 109.5 \\
\hline $\mathrm{H}(46 \mathrm{~A})-\mathrm{C}(46)-\mathrm{H}(46 \mathrm{~B})$ & 109.5 \\
\hline $\mathrm{C}(45)-\mathrm{C}(46)-\mathrm{H}(46 \mathrm{C})$ & 109.5 \\
\hline $\mathrm{H}(46 \mathrm{~A})-\mathrm{C}(46)-\mathrm{H}(46 \mathrm{C})$ & 109.5 \\
\hline $\mathrm{H}(46 \mathrm{~B})-\mathrm{C}(46)-\mathrm{H}(46 \mathrm{C})$ & 109.5 \\
\hline $\mathrm{C}(48)-\mathrm{C}(47)-\mathrm{P}(3)$ & $121.2(19)$ \\
\hline $\mathrm{C}(48)-\mathrm{C}(47)-\mathrm{H}(47 \mathrm{~A})$ & 107 \\
\hline $\mathrm{P}(3)-\mathrm{C}(47)-\mathrm{H}(47 \mathrm{~A})$ & 107 \\
\hline $\mathrm{C}(48)-\mathrm{C}(47)-\mathrm{H}(47 \mathrm{~B})$ & 107 \\
\hline $\mathrm{P}(3)-\mathrm{C}(47)-\mathrm{H}(47 \mathrm{~B})$ & 107 \\
\hline $\mathrm{H}(47 \mathrm{~A})-\mathrm{C}(47)-\mathrm{H}(47 \mathrm{~B})$ & 106.8 \\
\hline $\mathrm{C}(47)-\mathrm{C}(48)-\mathrm{H}(48 \mathrm{~A})$ & 109.5 \\
\hline $\mathrm{C}(47)-\mathrm{C}(48)-\mathrm{H}(48 \mathrm{~B})$ & 109.5 \\
\hline $\mathrm{H}(48 \mathrm{~A})-\mathrm{C}(48)-\mathrm{H}(48 \mathrm{~B})$ & 109.5 \\
\hline C(47)-C(48)-H(48C) & 109.5 \\
\hline $\mathrm{H}(48 \mathrm{~A})-\mathrm{C}(48)-\mathrm{H}(48 \mathrm{C})$ & 109.5 \\
\hline $\mathrm{H}(48 \mathrm{~B})-\mathrm{C}(48)-\mathrm{H}(48 \mathrm{C})$ & 109.5 \\
\hline $\mathrm{C}(50)-\mathrm{C}(49)-\mathrm{P}(3)$ & $107.9(15)$ \\
\hline $\mathrm{C}(50)-\mathrm{C}(49)-\mathrm{H}(49 \mathrm{~A})$ & 110.1 \\
\hline P(3)-C(49)-H(49A) & 110.1 \\
\hline $\mathrm{C}(50)-\mathrm{C}(49)-\mathrm{H}(49 \mathrm{~B})$ & 110.1 \\
\hline $\mathrm{P}(3)-\mathrm{C}(49)-\mathrm{H}(49 \mathrm{~B})$ & 110.1 \\
\hline
\end{tabular}




\begin{tabular}{|c|c|}
\hline $\mathrm{H}(49 \mathrm{~A})-\mathrm{C}(49)-\mathrm{H}(49 \mathrm{~B})$ & 108.4 \\
\hline $\mathrm{C}(49)-\mathrm{C}(50)-\mathrm{H}(50 \mathrm{~A})$ & 109.5 \\
\hline $\mathrm{C}(49)-\mathrm{C}(50)-\mathrm{H}(50 \mathrm{~B})$ & 109.5 \\
\hline $\mathrm{H}(50 \mathrm{~A})-\mathrm{C}(50)-\mathrm{H}(50 \mathrm{~B})$ & 109.5 \\
\hline C(49)-C(50)-H(50C) & 109.5 \\
\hline $\mathrm{H}(50 \mathrm{~A})-\mathrm{C}(50)-\mathrm{H}(50 \mathrm{C})$ & 109.5 \\
\hline $\mathrm{H}(50 \mathrm{~B})-\mathrm{C}(50)-\mathrm{H}(50 \mathrm{C})$ & 109.5 \\
\hline C(52)-C(51)-P(3) & $115.1(14)$ \\
\hline $\mathrm{C}(52)-\mathrm{C}(51)-\mathrm{H}(51 \mathrm{~A})$ & 108.5 \\
\hline $\mathrm{P}(3)-\mathrm{C}(51)-\mathrm{H}(51 \mathrm{~A})$ & 108.5 \\
\hline $\mathrm{C}(52)-\mathrm{C}(51)-\mathrm{H}(51 \mathrm{~B})$ & 108.5 \\
\hline $\mathrm{P}(3)-\mathrm{C}(51)-\mathrm{H}(51 \mathrm{~B})$ & 108.5 \\
\hline $\mathrm{H}(51 \mathrm{~A})-\mathrm{C}(51)-\mathrm{H}(51 \mathrm{~B})$ & 107.5 \\
\hline $\mathrm{C}(51)-\mathrm{C}(52)-\mathrm{H}(52 \mathrm{~A})$ & 109.5 \\
\hline $\mathrm{C}(51)-\mathrm{C}(52)-\mathrm{H}(52 \mathrm{~B})$ & 109.5 \\
\hline $\mathrm{H}(52 \mathrm{~A})-\mathrm{C}(52)-\mathrm{H}(52 \mathrm{~B})$ & 109.5 \\
\hline C(51)-C(52)-H(52C) & 109.5 \\
\hline $\mathrm{H}(52 \mathrm{~A})-\mathrm{C}(52)-\mathrm{H}(52 \mathrm{C})$ & 109.5 \\
\hline $\mathrm{H}(52 \mathrm{~B})-\mathrm{C}(52)-\mathrm{H}(52 \mathrm{C})$ & 109.5 \\
\hline C(54)-C(53)-P(4) & $116.1(16)$ \\
\hline $\mathrm{C}(54)-\mathrm{C}(53)-\mathrm{H}(53 \mathrm{~A})$ & 108.3 \\
\hline $\mathrm{P}(4)-\mathrm{C}(53)-\mathrm{H}(53 \mathrm{~A})$ & 108.3 \\
\hline $\mathrm{C}(54)-\mathrm{C}(53)-\mathrm{H}(53 \mathrm{~B})$ & 108.3 \\
\hline $\mathrm{P}(4)-\mathrm{C}(53)-\mathrm{H}(53 \mathrm{~B})$ & 108.3 \\
\hline $\mathrm{H}(53 \mathrm{~A})-\mathrm{C}(53)-\mathrm{H}(53 \mathrm{~B})$ & 107.4 \\
\hline C(53)-C(54)-H(54A) & 109.5 \\
\hline C(53)-C(54)-H(54B) & 109.5 \\
\hline $\mathrm{H}(54 \mathrm{~A})-\mathrm{C}(54)-\mathrm{H}(54 \mathrm{~B})$ & 109.5 \\
\hline C(53)-C(54)-H(54C) & 109.5 \\
\hline $\mathrm{H}(54 \mathrm{~A})-\mathrm{C}(54)-\mathrm{H}(54 \mathrm{C})$ & 109.5 \\
\hline $\mathrm{H}(54 \mathrm{~B})-\mathrm{C}(54)-\mathrm{H}(54 \mathrm{C})$ & 109.5 \\
\hline $\mathrm{C}(56)-\mathrm{C}(55)-\mathrm{P}(4)$ & $113.8(15)$ \\
\hline $\mathrm{C}(56)-\mathrm{C}(55)-\mathrm{H}(55 \mathrm{~A})$ & 108.8 \\
\hline $\mathrm{P}(4)-\mathrm{C}(55)-\mathrm{H}(55 \mathrm{~A})$ & 108.8 \\
\hline $\mathrm{C}(56)-\mathrm{C}(55)-\mathrm{H}(55 \mathrm{~B})$ & 108.8 \\
\hline $\mathrm{P}(4)-\mathrm{C}(55)-\mathrm{H}(55 \mathrm{~B})$ & 108.8 \\
\hline $\mathrm{H}(55 \mathrm{~A})-\mathrm{C}(55)-\mathrm{H}(55 \mathrm{~B})$ & 107.7 \\
\hline C(55)-C(56)-H(56A) & 109.5 \\
\hline $\mathrm{C}(55)-\mathrm{C}(56)-\mathrm{H}(56 \mathrm{~B})$ & 109.5 \\
\hline $\mathrm{H}(56 \mathrm{~A})-\mathrm{C}(56)-\mathrm{H}(56 \mathrm{~B})$ & 109.5 \\
\hline $\mathrm{C}(55)-\mathrm{C}(56)-\mathrm{H}(56 \mathrm{C})$ & 109.5 \\
\hline $\mathrm{H}(56 \mathrm{~A})-\mathrm{C}(56)-\mathrm{H}(56 \mathrm{C})$ & 109.5 \\
\hline $\mathrm{H}(56 \mathrm{~B})-\mathrm{C}(56)-\mathrm{H}(56 \mathrm{C})$ & 109.5 \\
\hline
\end{tabular}




$\begin{array}{lr}\mathrm{C}(58)-\mathrm{C}(57)-\mathrm{P}(4) & 112.0(12) \\ \mathrm{C}(58)-\mathrm{C}(57)-\mathrm{H}(57 \mathrm{~A}) & 109.2 \\ \mathrm{P}(4)-\mathrm{C}(57)-\mathrm{H}(57 \mathrm{~A}) & 109.2 \\ \mathrm{C}(58)-\mathrm{C}(57)-\mathrm{H}(57 \mathrm{~B}) & 109.2 \\ \mathrm{P}(4)-\mathrm{C}(57)-\mathrm{H}(57 \mathrm{~B}) & 109.2 \\ \mathrm{H}(57 \mathrm{~A})-\mathrm{C}(57)-\mathrm{H}(57 \mathrm{~B}) & 107.9 \\ \mathrm{C}(57)-\mathrm{C}(58)-\mathrm{H}(58 \mathrm{~A}) & 109.5 \\ \mathrm{C}(57)-\mathrm{C}(58)-\mathrm{H}(58 \mathrm{~B}) & 109.5 \\ \mathrm{H}(58 \mathrm{~A})-\mathrm{C}(58)-\mathrm{H}(58 \mathrm{~B}) & 109.5 \\ \mathrm{C}(57)-\mathrm{C}(58)-\mathrm{H}(58 \mathrm{C}) & 109.5 \\ \mathrm{H}(58 \mathrm{~A})-\mathrm{C}(58)-\mathrm{H}(58 \mathrm{C}) & 109.5 \\ \mathrm{H}(58 \mathrm{~B})-\mathrm{C}(58)-\mathrm{H}(58 \mathrm{C}) & 109.5 \\ \mathrm{~F}(3)-\mathrm{C}(63)-\mathrm{F}(2) & 98.8(19) \\ \mathrm{F}(3)-\mathrm{C}(63)-\mathrm{F}(1) & 107(3) \\ \mathrm{F}(2)-\mathrm{C}(63)-\mathrm{F}(1) & 105.1(19) \\ \mathrm{F}(3)-\mathrm{C}(63)-\mathrm{S}(1) & 116.3(17) \\ \mathrm{F}(2)-\mathrm{C}(63)-\mathrm{S}(1) & 114.6(16) \\ \mathrm{F}(1)-\mathrm{C}(63)-\mathrm{S}(1) & 113.8(16) \\ \mathrm{F}(5)-\mathrm{C}(64)-\mathrm{F}(6) & 101.1(13) \\ \mathrm{F}(5)-\mathrm{C}(64)-\mathrm{F}(4) & 112.6(16) \\ \mathrm{F}(6)-\mathrm{C}(64)-\mathrm{F}(4) & 112.1(15) \\ \mathrm{F}(5)-\mathrm{C}(64)-\mathrm{S}(2) & 115.5(12) \\ \mathrm{F}(6)-\mathrm{C}(64)-\mathrm{S}(2) & 112.0(12) \\ \mathrm{F}(4)-\mathrm{C}(64)-\mathrm{S}(2) & 103.9(12) \\ \mathrm{O}(67)-\mathrm{C}(65)-\mathrm{C}(66) \# 2 & 124.0(14) \\ \mathrm{O}(67)-\mathrm{C}(65)-\mathrm{C}(66) & 124.0(14) \\ \mathrm{C}(66) \# 2-\mathrm{C}(65)-\mathrm{C}(66) & 109.5 \\ \mathrm{C}(65)-\mathrm{C}(66)-\mathrm{H}(66 \mathrm{~A}) & 109.5 \\ \mathrm{C}(65)-\mathrm{C}(66)-\mathrm{H}(66 \mathrm{~B}) & 109.5 \\ \mathrm{H}(66 \mathrm{~A})-\mathrm{C}(66)-\mathrm{H}(66 \mathrm{~B}) & 109.5 \\ \mathrm{C}(65)-\mathrm{C}(66)-\mathrm{H}(66 \mathrm{C}) & 109.5 \\ \mathrm{H}(66 \mathrm{~A})-\mathrm{C}(66)-\mathrm{H}(66 \mathrm{C}) & 109.5 \\ \mathrm{H}(66 \mathrm{~B})-\mathrm{C}(66)-\mathrm{H}(66 \mathrm{C}) & \\ \end{array}$

Symmetry transformations used to generate equivalent atoms:

$\# 1-x+3 / 2,-y+3 / 2,-z+1 \quad \# 2-x+1, y,-z+1 / 2$

Table 11. Atomic coordinates $\left(\times 10^{4}\right)$ and equivalent isotropic displacement parameters $\left(\AA^{2} \times 10^{3}\right)$ for BT3'. U(eq) is defined as one third of the trace of the orthogonalized $\mathrm{U}^{\mathrm{ij}}$ tensor.

\begin{tabular}{ccccc}
\hline & $\mathrm{x}$ & $\mathrm{y}$ & $\mathrm{z}$ & $\mathrm{U}(\mathrm{eq})$ \\
\hline $\operatorname{Pt}(1)$ & $4111(1)$ & $3450(1)$ & $5479(1)$ & $128(1)$
\end{tabular}




\begin{tabular}{|c|c|c|c|c|}
\hline $\operatorname{Pt}(2)$ & $3825(1)$ & $4802(1)$ & $1183(1)$ & $108(1)$ \\
\hline $\mathrm{P}(1)$ & $3645(5)$ & 4940(5) & $319(8)$ & $152(5)$ \\
\hline $\mathrm{P}(2)$ & $4263(5)$ & $5280(4)$ & $1279(8)$ & $140(5)$ \\
\hline $\mathrm{P}(3)$ & $4548(4)$ & $3879(4)$ & $5824(7)$ & $125(5)$ \\
\hline $\mathrm{P}(4)$ & $4116(10)$ & $3020(9)$ & $6198(13)$ & $311(18)$ \\
\hline $\mathrm{O}(1)$ & $4167(10)$ & $3740(9)$ & $4713(16)$ & $140(10)$ \\
\hline $\mathrm{O}(51)$ & $3881(10)$ & $4670(8)$ & 1981(13) & 141(9) \\
\hline$C(17)$ & 1964(8) & 1988(8) & $1729(11)$ & $94(10)$ \\
\hline $\mathrm{C}(8)$ & $1732(8)$ & $1645(9)$ & $1756(13)$ & $117(11)$ \\
\hline $\mathrm{C}(9)$ & $1765(8)$ & $1490(7)$ & $2304(15)$ & 111(11) \\
\hline$C(10)$ & 2017(8) & 1737(9) & $2615(10)$ & 97(9) \\
\hline $\mathrm{N}(3)$ & $2140(7)$ & $2044(7)$ & $2259(12)$ & $98(9)$ \\
\hline$C(11)$ & $2113(11)$ & $1678(11)$ & $3180(20)$ & $88(9)$ \\
\hline$C(12)$ & $2350(7)$ & 1931(7) & $3466(12)$ & $95(9)$ \\
\hline$C(13)$ & $2435(8)$ & 1882(7) & $4042(12)$ & $99(9)$ \\
\hline$C(14)$ & $2696(8)$ & 2183(8) & $4204(10)$ & $104(10)$ \\
\hline$C(15)$ & 2771(7) & $2419(6)$ & $3728(13)$ & 91(9) \\
\hline $\mathrm{N}(4)$ & $2558(7)$ & $2263(7)$ & $3272(10)$ & $89(8)$ \\
\hline$C(16)$ & $2979(11)$ & $2748(11)$ & $3670(20)$ & $87(9)$ \\
\hline$C(18)$ & $3867(7)$ & 2913(9) & $4577(15)$ & $128(11)$ \\
\hline$C(19)$ & $3619(9)$ & 2784(8) & $4156(12)$ & $108(10)$ \\
\hline$C(20)$ & $3220(8)$ & 2851(8) & $4195(12)$ & 101(10) \\
\hline$C(21)$ & $3069(7)$ & $3047(9)$ & $4654(15)$ & $127(11)$ \\
\hline$C(22)$ & $3317(10)$ & $3177(9)$ & $5075(12)$ & $140(12)$ \\
\hline $\mathrm{N}(2)$ & $3716(10)$ & $3110(9)$ & $5036(12)$ & 141(11) \\
\hline$C(23)$ & $3610(40)$ & $3460(30)$ & $6730(50)$ & $420(40)$ \\
\hline$C(24)$ & $4643(15)$ & $3925(17)$ & $6530(20)$ & $149(12)$ \\
\hline$C(25)$ & $4925(18)$ & $4214(18)$ & $6700(30)$ & $180(20)$ \\
\hline$C(26)$ & $4443(12)$ & $4363(13)$ & $5550(20)$ & $121(11)$ \\
\hline$C(27)$ & $4059(16)$ & $4499(17)$ & $5910(30)$ & $170(19)$ \\
\hline $\mathrm{C}(40)$ & $4263(15)$ & $5484(17)$ & $1890(30)$ & $183(14)$ \\
\hline$C(41)$ & $3900(20)$ & $5700(20)$ & $2140(30)$ & $240(30)$ \\
\hline$C(43)$ & $3262(19)$ & $4591(18)$ & $40(30)$ & $181(15)$ \\
\hline$C(45)$ & $4090(20)$ & $4880(20)$ & $-230(30)$ & $200(20)$ \\
\hline$C(46)$ & $4956(19)$ & $5310(20)$ & 1920(40) & $290(30)$ \\
\hline$C(50)$ & $4065(14)$ & $4382(13)$ & $2158(13)$ & $143(12)$ \\
\hline$C(56)$ & $3938(11)$ & $4047(11)$ & $3889(10)$ & $143(11)$ \\
\hline$C(57)$ & $4203(9)$ & $3866(8)$ & $3534(16)$ & $135(11)$ \\
\hline $\mathrm{C}(58)$ & $4221(8)$ & 3972(9) & $2975(15)$ & $134(11)$ \\
\hline$C(59)$ & $3974(10)$ & $4260(10)$ & $2770(11)$ & $125(10)$ \\
\hline$C(60)$ & $3709(8)$ & 4441(7) & $3124(16)$ & $124(11)$ \\
\hline$C(61)$ & 3691(9) & 4335(9) & $3684(15)$ & $126(10)$ \\
\hline$C(62)$ & $3900(15)$ & $3955(16)$ & $4520(14)$ & $154(13)$ \\
\hline
\end{tabular}




\begin{tabular}{lrrrr}
$\mathrm{O}(63)$ & $3567(15)$ & $4032(15)$ & $4770(20)$ & $213(17)$ \\
$\mathrm{O}(65)$ & $4248(15)$ & $4151(12)$ & $1837(19)$ & $211(17)$ \\
$\mathrm{C}(67)$ & $3097(7)$ & $4368(6)$ & $1536(12)$ & $89(9)$ \\
$\mathrm{C}(68)$ & $2900(6)$ & $4032(7)$ & $1695(12)$ & $92(10)$ \\
$\mathrm{C}(69)$ & $3049(7)$ & $3668(6)$ & $1556(12)$ & $90(10)$ \\
$\mathrm{C}(70)$ & $3396(8)$ & $3640(5)$ & $1257(12)$ & $100(11)$ \\
$\mathrm{C}(71)$ & $3593(6)$ & $3977(7)$ & $1099(11)$ & $102(11)$ \\
$\mathrm{N}(1)$ & $3444(7)$ & $4341(6)$ & $1238(11)$ & $103(8)$ \\
$\mathrm{C}(74)$ & $4639(11)$ & $2850(20)$ & $6330(30)$ & $350(20)$ \\
$\mathrm{C}(73)$ & $4250(20)$ & $4540(20)$ & $-70(30)$ & $230(30)$ \\
$\mathrm{C}(108)$ & $5218(19)$ & $3473(19)$ & $5190(30)$ & $220(30)$ \\
$\mathrm{C}(109)$ & $5034(14)$ & $3785(17)$ & $5550(30)$ & $159(13)$ \\
$\mathrm{C}(110)$ & $3030(20)$ & $4800(30)$ & $-490(30)$ & $230(30)$ \\
$\mathrm{C}(112)$ & $3430(20)$ & $5397(17)$ & $120(30)$ & $180(14)$ \\
$\mathrm{C}(113)$ & $3100(20)$ & $5470(20)$ & $590(30)$ & $240(30)$ \\
$\mathrm{C}(114)$ & $4370(20)$ & $5666(17)$ & $650(20)$ & $240(30)$ \\
$\mathrm{C}(115)$ & $4759(16)$ & $5018(18)$ & $1520(30)$ & $193(15)$ \\
$\mathrm{C}(116)$ & $4720(20)$ & $5910(18)$ & $860(30)$ & $240(30)$ \\
$\mathrm{C}(117)$ & $3810(20)$ & $3080(30)$ & $6940(30)$ & $420(40)$ \\
$\mathrm{C}(118)$ & $3727(17)$ & $2629(17)$ & $6070(40)$ & $340(30)$ \\
$\mathrm{C}(119)$ & $3860(30)$ & $2220(16)$ & $6260(50)$ & $410(40)$ \\
$\mathrm{C}(120)$ & $4680(30)$ & $2700(40)$ & $6940(30)$ & $440(40)$ \\
$\mathrm{S}(1 \mathrm{~S})$ & $4404(11)$ & $1684(9)$ & $7280(20)$ & $390(20)$ \\
$\mathrm{O}(1 \mathrm{~S})$ & $4598(16)$ & $1360(14)$ & $7460(30)$ & $450(30)$ \\
$\mathrm{O}(2 \mathrm{~S})$ & $4021(16)$ & $1610(20)$ & $7170(30)$ & $450(30)$ \\
$\mathrm{O}(3 \mathrm{~S})$ & $4600(20)$ & $1894(18)$ & $6900(30)$ & $440(30)$ \\
$\mathrm{C}(1 \mathrm{~S})$ & $4375(14)$ & $1943(13)$ & $7830(20)$ & $420(30)$ \\
$\mathrm{F}(1 \mathrm{~S})$ & $4688(18)$ & $2050(20)$ & $8040(30)$ & $470(30)$ \\
$\mathrm{F}(2 \mathrm{~S})$ & $4180(20)$ & $2250(15)$ & $7780(30)$ & $450(30)$ \\
$\mathrm{F}(3 \mathrm{~S})$ & $4210(20)$ & $1783(19)$ & $8240(20)$ & $470(30)$ \\
$\mathrm{S}(2 \mathrm{~S})$ & $5941(11)$ & $5422(12)$ & $2096(16)$ & $359(19)$ \\
$\mathrm{O}(4 \mathrm{~S})$ & $6030(15)$ & $5130(14)$ & $1730(20)$ & $290(20)$ \\
$\mathrm{O}(5 \mathrm{~S})$ & $5640(20)$ & $5340(20)$ & $2440(30)$ & $410(30)$ \\
$\mathrm{O}(6 \mathrm{~S})$ & $6260(19)$ & $5560(20)$ & $2370(30)$ & $430(30)$ \\
$\mathrm{C}(2 \mathrm{~S})$ & $5798(13)$ & $5783(14)$ & $1734(19)$ & $370(20)$ \\
$\mathrm{F}(4 \mathrm{~S})$ & $5518(18)$ & $5730(18)$ & $1410(30)$ & $400(20)$ \\
$\mathrm{F}(5 \mathrm{~S})$ & $5680(20)$ & $6075(14)$ & $2000(30)$ & $410(20)$ \\
$\mathrm{F}(6 \mathrm{~S})$ & $6040(19)$ & $5947(16)$ & $1420(30)$ & $380(20)$ \\
\hline & & & & \\
\hline & & &
\end{tabular}

Table 12. Bond lengths $[\AA]$ and angles $\left[{ }^{\circ}\right]$ for BT3'.

$\operatorname{Pt}(1)-\mathrm{N}(2)$




\begin{tabular}{|c|c|}
\hline $\mathrm{Pt}(1)-\mathrm{O}(1)$ & $2.10(4)$ \\
\hline $\mathrm{Pt}(1)-\mathrm{P}(3)$ & $2.255(13)$ \\
\hline $\mathrm{Pt}(1)-\mathrm{P}(4)$ & $2.27(2)$ \\
\hline $\operatorname{Pt}(2)-\mathrm{O}(51)$ & $1.97(3)$ \\
\hline $\operatorname{Pt}(2)-\mathrm{N}(1)$ & $2.057(19)$ \\
\hline $\mathrm{Pt}(2)-\mathrm{P}(1)$ & $2.210(19)$ \\
\hline $\mathrm{Pt}(2)-\mathrm{P}(2)$ & $2.236(13)$ \\
\hline $\mathrm{P}(1)-\mathrm{C}(112)$ & $1.80(6)$ \\
\hline $\mathrm{P}(1)-\mathrm{C}(43)$ & $1.89(6)$ \\
\hline $\mathrm{P}(1)-\mathrm{C}(45)$ & $2.02(7)$ \\
\hline$P(2)-C(40)$ & $1.61(6)$ \\
\hline $\mathrm{P}(2)-\mathrm{C}(115)$ & $2.00(6)$ \\
\hline $\mathrm{P}(2)-\mathrm{C}(114)$ & $2.04(2)$ \\
\hline $\mathrm{P}(3)-\mathrm{C}(24)$ & $1.72(6)$ \\
\hline $\mathrm{P}(3)-\mathrm{C}(109)$ & $1.82(5)$ \\
\hline $\mathrm{P}(3)-\mathrm{C}(26)$ & $1.81(5)$ \\
\hline $\mathrm{P}(4)-C(74)$ & $1.91(2)$ \\
\hline P(4)-C(118) & $1.91(2)$ \\
\hline$P(4)-C(117)$ & $2.07(2)$ \\
\hline $\mathrm{O}(1)-\mathrm{C}(62)$ & $1.26(2)$ \\
\hline $\mathrm{O}(51)-\mathrm{C}(50)$ & $1.25(2)$ \\
\hline$C(17)-C(16) \# 1$ & $1.33(4)$ \\
\hline$C(17)-C(8)$ & 1.42 \\
\hline $\mathrm{C}(17)-\mathrm{N}(3)$ & 1.42 \\
\hline C(8)-C(9) & 1.42 \\
\hline C(8)-H(8) & 0.95 \\
\hline$C(9)-C(10)$ & 1.42 \\
\hline C(9)-H(9) & 0.95 \\
\hline $\mathrm{C}(10)-\mathrm{N}(3)$ & 1.42 \\
\hline$C(10)-C(11)$ & $1.42(5)$ \\
\hline$C(11)-C(12)$ & $1.36(4)$ \\
\hline $\mathrm{C}(11)-\mathrm{C}(69) \# 1$ & $1.45(4)$ \\
\hline$C(12)-C(13)$ & 1.42 \\
\hline $\mathrm{C}(12)-\mathrm{N}(4)$ & 1.42 \\
\hline$C(13)-C(14)$ & 1.42 \\
\hline $\mathrm{C}(13)-\mathrm{H}(13)$ & 0.95 \\
\hline$C(14)-C(15)$ & 1.42 \\
\hline C(14)-H(14) & 0.95 \\
\hline$C(15)-C(16)$ & $1.34(4)$ \\
\hline $\mathrm{C}(15)-\mathrm{N}(4)$ & 1.42 \\
\hline N(4)-H(4) & 0.88 \\
\hline$C(16)-C(20)$ & $1.55(5)$ \\
\hline $\mathrm{C}(18)-\mathrm{C}(19)$ & 1.39 \\
\hline
\end{tabular}




\begin{tabular}{|c|c|}
\hline $\mathrm{C}(18)-\mathrm{N}(2)$ & 1.39 \\
\hline C(18)-H(18) & 0.95 \\
\hline C(19)-C(20) & 1.39 \\
\hline C(19)-H(19) & 0.95 \\
\hline C(20)-C(21) & 1.39 \\
\hline$C(21)-C(22)$ & 1.39 \\
\hline $\mathrm{C}(21)-\mathrm{H}(21)$ & 0.95 \\
\hline $\mathrm{C}(22)-\mathrm{N}(2)$ & 1.39 \\
\hline C(22)-H(22) & 0.95 \\
\hline$C(23)-C(117)$ & $1.54(2)$ \\
\hline $\mathrm{C}(23)-\mathrm{H}(23 \mathrm{~A})$ & 0.9972 \\
\hline $\mathrm{C}(23)-\mathrm{H}(23 \mathrm{~B})$ & 0.9995 \\
\hline $\mathrm{C}(23)-\mathrm{H}(23 \mathrm{C})$ & 0.9981 \\
\hline$C(24)-C(25)$ & $1.45(6)$ \\
\hline $\mathrm{C}(24)-\mathrm{H}(24 \mathrm{~A})$ & 0.99 \\
\hline C(24)-H(24B) & 0.99 \\
\hline $\mathrm{C}(25)-\mathrm{H}(25 \mathrm{~A})$ & 0.98 \\
\hline $\mathrm{C}(25)-\mathrm{H}(25 \mathrm{~B})$ & 0.98 \\
\hline $\mathrm{C}(25)-\mathrm{H}(25 \mathrm{C})$ & 0.98 \\
\hline$C(26)-C(27)$ & $1.63(7)$ \\
\hline $\mathrm{C}(26)-\mathrm{H}(26 \mathrm{~A})$ & 0.99 \\
\hline $\mathrm{C}(26)-\mathrm{H}(26 \mathrm{~B})$ & 0.99 \\
\hline $\mathrm{C}(27)-\mathrm{H}(27 \mathrm{~A})$ & 1.0126 \\
\hline C(27)-H(27B) & 1.014 \\
\hline $\mathrm{C}(27)-\mathrm{H}(27 \mathrm{C})$ & 1.0134 \\
\hline $\mathrm{C}(40)-\mathrm{C}(41)$ & $1.56(2)$ \\
\hline $\mathrm{C}(40)-\mathrm{H}(40 \mathrm{~A})$ & 0.99 \\
\hline $\mathrm{C}(40)-\mathrm{H}(40 \mathrm{~B})$ & 0.99 \\
\hline $\mathrm{C}(41)-\mathrm{H}(41 \mathrm{~A})$ & 0.9939 \\
\hline $\mathrm{C}(41)-\mathrm{H}(41 \mathrm{~B})$ & 0.9946 \\
\hline $\mathrm{C}(41)-\mathrm{H}(41 \mathrm{C})$ & 0.994 \\
\hline C(43)-C(110) & $1.67(9)$ \\
\hline $\mathrm{C}(43)-\mathrm{H}(43 \mathrm{~A})$ & 0.99 \\
\hline C(43)-H(43B) & 0.99 \\
\hline$C(45)-C(73)$ & $1.34(8)$ \\
\hline $\mathrm{C}(45)-\mathrm{H}(45 \mathrm{~A})$ & 0.99 \\
\hline $\mathrm{C}(45)-\mathrm{H}(45 \mathrm{~B})$ & 0.99 \\
\hline $\mathrm{C}(46)-\mathrm{C}(115)$ & $1.55(2)$ \\
\hline $\mathrm{C}(46)-\mathrm{H}(46 \mathrm{~A})$ & 0.9838 \\
\hline $\mathrm{C}(46)-\mathrm{H}(46 \mathrm{~B})$ & 0.9838 \\
\hline $\mathrm{C}(46)-\mathrm{H}(46 \mathrm{C})$ & 0.9839 \\
\hline $\mathrm{C}(50)-\mathrm{O}(65)$ & $1.27(2)$ \\
\hline $\mathrm{C}(50)-\mathrm{C}(59)$ & $1.56(2)$ \\
\hline
\end{tabular}




\begin{tabular}{|c|c|}
\hline $\mathrm{C}(56)-\mathrm{C}(57)$ & 1.39 \\
\hline$C(56)-C(61)$ & 1.39 \\
\hline$C(56)-C(62)$ & $1.55(2)$ \\
\hline C(57)-C(58) & 1.39 \\
\hline C(57)-H(57) & 0.95 \\
\hline C(58)-C(59) & 1.39 \\
\hline $\mathrm{C}(58)-\mathrm{H}(58)$ & 0.95 \\
\hline C(59)-C(60) & 1.39 \\
\hline $\mathrm{C}(60)-\mathrm{C}(61)$ & 1.39 \\
\hline $\mathrm{C}(60)-\mathrm{H}(60)$ & 0.95 \\
\hline $\mathrm{C}(61)-\mathrm{H}(61)$ & 0.95 \\
\hline $\mathrm{C}(62)-\mathrm{O}(63)$ & $1.32(6)$ \\
\hline$C(67)-C(68)$ & 1.39 \\
\hline $\mathrm{C}(67)-\mathrm{N}(1)$ & 1.39 \\
\hline $\mathrm{C}(67)-\mathrm{H}(67)$ & 0.95 \\
\hline C(68)-C(69) & 1.39 \\
\hline $\mathrm{C}(68)-\mathrm{H}(68)$ & 0.95 \\
\hline C(69)-C(70) & 1.39 \\
\hline$C(70)-C(71)$ & 1.39 \\
\hline C(70)-H(70) & 0.95 \\
\hline $\mathrm{C}(71)-\mathrm{N}(1)$ & 1.39 \\
\hline $\mathrm{C}(71)-\mathrm{H}(71)$ & 0.95 \\
\hline$C(74)-C(120)$ & $1.55(2)$ \\
\hline $\mathrm{C}(74)-\mathrm{H}(74 \mathrm{~A})$ & 0.99 \\
\hline $\mathrm{C}(74)-\mathrm{H}(74 \mathrm{~B})$ & 0.99 \\
\hline $\mathrm{C}(73)-\mathrm{H}(73 \mathrm{~A})$ & 0.95 \\
\hline C(73)-H(73B) & 0.95 \\
\hline $\mathrm{C}(108)-\mathrm{C}(109)$ & $1.52(2)$ \\
\hline $\mathrm{C}(108)-\mathrm{H}(10 \mathrm{~A})$ & 0.9872 \\
\hline $\mathrm{C}(108)-\mathrm{H}(10 \mathrm{~B})$ & 0.9868 \\
\hline $\mathrm{C}(108)-\mathrm{H}(10 \mathrm{C})$ & 0.9866 \\
\hline $\mathrm{C}(109)-\mathrm{H}(10 \mathrm{D})$ & 0.99 \\
\hline $\mathrm{C}(109)-\mathrm{H}(10 \mathrm{E})$ & 0.99 \\
\hline $\mathrm{C}(110)-\mathrm{H}(11 \mathrm{~A})$ & 1.0093 \\
\hline $\mathrm{C}(110)-\mathrm{H}(11 \mathrm{~B})$ & 1.0096 \\
\hline $\mathrm{C}(110)-\mathrm{H}(11 \mathrm{C})$ & 1.0102 \\
\hline $\mathrm{C}(112)-\mathrm{C}(113)$ & $1.61(9)$ \\
\hline $\mathrm{C}(112)-\mathrm{H}(11 \mathrm{D})$ & 0.99 \\
\hline $\mathrm{C}(112)-\mathrm{H}(11 \mathrm{E})$ & 0.99 \\
\hline $\mathrm{C}(113)-\mathrm{H}(11 \mathrm{~F})$ & 1.0391 \\
\hline $\mathrm{C}(113)-\mathrm{H}(11 \mathrm{G})$ & 1.0376 \\
\hline $\mathrm{C}(113)-\mathrm{H}(11 \mathrm{H})$ & 1.036 \\
\hline $\mathrm{C}(114)-\mathrm{C}(116)$ & $1.55(2)$ \\
\hline
\end{tabular}




\begin{tabular}{|c|c|}
\hline C(114)-H(11I) & 0.99 \\
\hline $\mathrm{C}(114)-\mathrm{H}(11 \mathrm{~J})$ & 0.99 \\
\hline $\mathrm{C}(115)-\mathrm{H}(11 \mathrm{~K})$ & 0.99 \\
\hline $\mathrm{C}(115)-\mathrm{H}(11 \mathrm{~L})$ & 0.99 \\
\hline $\mathrm{C}(116)-\mathrm{H}(11 \mathrm{M})$ & 0.9903 \\
\hline $\mathrm{C}(116)-\mathrm{H}(11 \mathrm{~N})$ & 0.9903 \\
\hline $\mathrm{C}(116)-\mathrm{H}(11 \mathrm{O})$ & 0.99 \\
\hline $\mathrm{C}(117)-\mathrm{H}(11 \mathrm{P})$ & 0.99 \\
\hline C(117)-H(11Q) & 0.99 \\
\hline$C(118)-C(119)$ & $1.55(2)$ \\
\hline $\mathrm{C}(118)-\mathrm{H}(11 \mathrm{R})$ & 0.99 \\
\hline C(118)-H(11S) & 0.99 \\
\hline $\mathrm{C}(119)-\mathrm{H}(11 \mathrm{~T})$ & 0.9946 \\
\hline C(119)-H(11U) & 0.9952 \\
\hline C(119)-H(11V) & 0.9948 \\
\hline $\mathrm{C}(120)-\mathrm{H}(12 \mathrm{~A})$ & 1.113 \\
\hline $\mathrm{C}(120)-\mathrm{H}(12 \mathrm{~B})$ & 1.1285 \\
\hline $\mathrm{C}(120)-\mathrm{H}(12 \mathrm{C})$ & 1.1366 \\
\hline $\mathrm{S}(1 \mathrm{~S})-\mathrm{O}(3 \mathrm{~S})$ & $1.34(4)$ \\
\hline $\mathrm{S}(1 \mathrm{~S})-\mathrm{O}(2 \mathrm{~S})$ & $1.36(3)$ \\
\hline $\mathrm{S}(1 \mathrm{~S})-\mathrm{O}(1 \mathrm{~S})$ & $1.37(3)$ \\
\hline $\mathrm{S}(1 \mathrm{~S})-\mathrm{C}(1 \mathrm{~S})$ & $1.59(2)$ \\
\hline $\mathrm{C}(1 \mathrm{~S})-\mathrm{F}(1 \mathrm{~S})$ & $1.24(3)$ \\
\hline$C(1 S)-F(2 S)$ & $1.25(3)$ \\
\hline $\mathrm{C}(1 \mathrm{~S})-\mathrm{F}(3 \mathrm{~S})$ & $1.25(3)$ \\
\hline $\mathrm{S}(2 \mathrm{~S})-\mathrm{O}(4 \mathrm{~S})$ & $1.36(3)$ \\
\hline $\mathrm{S}(2 \mathrm{~S})-\mathrm{O}(5 \mathrm{~S})$ & $1.36(3)$ \\
\hline $\mathrm{S}(2 \mathrm{~S})-\mathrm{O}(6 \mathrm{~S})$ & $1.37(4)$ \\
\hline $\mathrm{S}(2 \mathrm{~S})-\mathrm{C}(2 \mathrm{~S})$ & $1.59(3)$ \\
\hline $\mathrm{C}(2 \mathrm{~S})-\mathrm{F}(4 \mathrm{~S})$ & $1.25(3)$ \\
\hline $\mathrm{C}(2 \mathrm{~S})-\mathrm{F}(6 \mathrm{~S})$ & $1.25(3)$ \\
\hline$C(2 S)-F(5 S)$ & $1.26(3)$ \\
\hline $\mathrm{N}(2)-\operatorname{Pt}(1)-\mathrm{O}(1)$ & $83.1(11)$ \\
\hline N(2)-Pt(1)-P(3) & $170.2(9)$ \\
\hline $\mathrm{O}(1)-\mathrm{Pt}(1)-\mathrm{P}(3)$ & 87.1(9) \\
\hline $\mathrm{N}(2)-\operatorname{Pt}(1)-\mathrm{P}(4)$ & $91.5(10)$ \\
\hline $\mathrm{O}(1)-\mathrm{Pt}(1)-\mathrm{P}(4)$ & $166.6(16)$ \\
\hline P(3)-Pt(1)-P(4) & $98.2(7)$ \\
\hline $\mathrm{O}(51)-\mathrm{Pt}(2)-\mathrm{N}(1)$ & $79.8(11)$ \\
\hline $\mathrm{O}(51)-\mathrm{Pt}(2)-\mathrm{P}(1)$ & $169.2(11)$ \\
\hline $\mathrm{N}(1)-\mathrm{Pt}(2)-\mathrm{P}(1)$ & $92.7(9)$ \\
\hline $\mathrm{O}(51)-\mathrm{Pt}(2)-\mathrm{P}(2)$ & $90.1(10)$ \\
\hline
\end{tabular}




\begin{tabular}{|c|c|}
\hline $\mathrm{N}(1)-\mathrm{Pt}(2)-\mathrm{P}(2)$ & $169.9(9)$ \\
\hline $\mathrm{P}(1)-\mathrm{Pt}(2)-\mathrm{P}(2)$ & $97.4(6)$ \\
\hline $\mathrm{C}(112)-\mathrm{P}(1)-\mathrm{C}(43)$ & $100(3)$ \\
\hline $\mathrm{C}(112)-\mathrm{P}(1)-\mathrm{C}(45)$ & $102(3)$ \\
\hline C(43)-P(1)-C(45) & $103(3)$ \\
\hline $\mathrm{C}(112)-\mathrm{P}(1)-\mathrm{Pt}(2)$ & $124(3)$ \\
\hline $\mathrm{C}(43)-\mathrm{P}(1)-\mathrm{Pt}(2)$ & $113(2)$ \\
\hline C(45)-P(1)-Pt(2) & $112(2)$ \\
\hline $\mathrm{C}(40)-\mathrm{P}(2)-\mathrm{C}(115)$ & $87(3)$ \\
\hline $\mathrm{C}(40)-\mathrm{P}(2)-\mathrm{C}(114)$ & $113(3)$ \\
\hline $\mathrm{C}(115)-\mathrm{P}(2)-\mathrm{C}(114)$ & $111(3)$ \\
\hline $\mathrm{C}(40)-\mathrm{P}(2)-\mathrm{Pt}(2)$ & $114(2)$ \\
\hline $\mathrm{C}(115)-\mathrm{P}(2)-\mathrm{Pt}(2)$ & $105.6(17)$ \\
\hline C(114)-P(2)-Pt(2) & 121.1(16) \\
\hline C(24)-P(3)-C(109) & $101(3)$ \\
\hline $\mathrm{C}(24)-\mathrm{P}(3)-\mathrm{C}(26)$ & $107(3)$ \\
\hline C(109)-P(3)-C(26) & $103(3)$ \\
\hline $\mathrm{C}(24)-\mathrm{P}(3)-\mathrm{Pt}(1)$ & $123.0(19)$ \\
\hline C(109)-P(3)-Pt(1) & 111.2(19) \\
\hline C(26)-P(3)-Pt(1) & $109.6(14)$ \\
\hline $\mathrm{C}(74)-\mathrm{P}(4)-\mathrm{C}(118)$ & $118.1(18)$ \\
\hline $\mathrm{C}(74)-\mathrm{P}(4)-\mathrm{C}(117)$ & $111.0(15)$ \\
\hline C(118)-P(4)-C(117) & $82(4)$ \\
\hline $\mathrm{C}(74)-\mathrm{P}(4)-\mathrm{Pt}(1)$ & $109(2)$ \\
\hline C(118)-P(4)-Pt(1) & $109(2)$ \\
\hline $\mathrm{C}(117)-\mathrm{P}(4)-\mathrm{Pt}(1)$ & $126(3)$ \\
\hline C(62)-O(1)-Pt(1) & $122(3)$ \\
\hline $\mathrm{C}(50)-\mathrm{O}(51)-\mathrm{Pt}(2)$ & $124(2)$ \\
\hline $\mathrm{C}(16) \# 1-\mathrm{C}(17)-\mathrm{C}(8)$ & $133(3)$ \\
\hline C(16)\#1-C(17)-N(3) & $119(3)$ \\
\hline $\mathrm{C}(8)-\mathrm{C}(17)-\mathrm{N}(3)$ & 108 \\
\hline $\mathrm{C}(17)-\mathrm{C}(8)-\mathrm{C}(9)$ & 108 \\
\hline $\mathrm{C}(17)-\mathrm{C}(8)-\mathrm{H}(8)$ & 126 \\
\hline C(9)-C(8)-H(8) & 126 \\
\hline $\mathrm{C}(10)-\mathrm{C}(9)-\mathrm{C}(8)$ & 108 \\
\hline $\mathrm{C}(10)-\mathrm{C}(9)-\mathrm{H}(9)$ & 126 \\
\hline C(8)-C(9)-H(9) & 126 \\
\hline N(3)-C(10)-C(9) & 108 \\
\hline $\mathrm{N}(3)-\mathrm{C}(10)-\mathrm{C}(11)$ & $128(3)$ \\
\hline $\mathrm{C}(9)-\mathrm{C}(10)-\mathrm{C}(11)$ & $124(3)$ \\
\hline $\mathrm{C}(10)-\mathrm{N}(3)-\mathrm{C}(17)$ & 108 \\
\hline $\mathrm{C}(12)-\mathrm{C}(11)-\mathrm{C}(10)$ & $121(3)$ \\
\hline $\mathrm{C}(12)-\mathrm{C}(11)-\mathrm{C}(69) \# 1$ & $123(9)$ \\
\hline
\end{tabular}




\begin{tabular}{|c|c|}
\hline$C(10)-C(11)-C(69) \# 1$ & $116(8)$ \\
\hline$C(11)-C(12)-C(13)$ & $122(3)$ \\
\hline $\mathrm{C}(11)-\mathrm{C}(12)-\mathrm{N}(4)$ & $130(3)$ \\
\hline $\mathrm{C}(13)-\mathrm{C}(12)-\mathrm{N}(4)$ & 108 \\
\hline$C(14)-C(13)-C(12)$ & 108 \\
\hline $\mathrm{C}(14)-\mathrm{C}(13)-\mathrm{H}(13)$ & 126 \\
\hline $\mathrm{C}(12)-\mathrm{C}(13)-\mathrm{H}(13)$ & 126 \\
\hline $\mathrm{C}(13)-\mathrm{C}(14)-\mathrm{C}(15)$ & 108 \\
\hline $\mathrm{C}(13)-\mathrm{C}(14)-\mathrm{H}(14)$ & 126 \\
\hline $\mathrm{C}(15)-\mathrm{C}(14)-\mathrm{H}(14)$ & 126 \\
\hline $\mathrm{C}(16)-\mathrm{C}(15)-\mathrm{N}(4)$ & $121(3)$ \\
\hline$C(16)-C(15)-C(14)$ & 131(3) \\
\hline N(4)-C(15)-C(14) & 108 \\
\hline $\mathrm{C}(15)-\mathrm{N}(4)-\mathrm{C}(12)$ & 108 \\
\hline C(15)-N(4)-H(4) & 126 \\
\hline $\mathrm{C}(12)-\mathrm{N}(4)-\mathrm{H}(4)$ & 126 \\
\hline $\mathrm{C}(17) \# 1-\mathrm{C}(16)-\mathrm{C}(15)$ & $137(4)$ \\
\hline$C(17) \# 1-C(16)-C(20)$ & $110(3)$ \\
\hline$C(15)-C(16)-C(20)$ & $113(3)$ \\
\hline $\mathrm{C}(19)-\mathrm{C}(18)-\mathrm{N}(2)$ & 120 \\
\hline $\mathrm{C}(19)-\mathrm{C}(18)-\mathrm{H}(18)$ & 120 \\
\hline $\mathrm{N}(2)-\mathrm{C}(18)-\mathrm{H}(18)$ & 120 \\
\hline$C(20)-C(19)-C(18)$ & 120 \\
\hline $\mathrm{C}(20)-\mathrm{C}(19)-\mathrm{H}(19)$ & 120 \\
\hline $\mathrm{C}(18)-\mathrm{C}(19)-\mathrm{H}(19)$ & 120 \\
\hline$C(21)-C(20)-C(19)$ & 120 \\
\hline$C(21)-C(20)-C(16)$ & $124(3)$ \\
\hline$C(19)-C(20)-C(16)$ & $116(3)$ \\
\hline$C(22)-C(21)-C(20)$ & 120 \\
\hline $\mathrm{C}(22)-\mathrm{C}(21)-\mathrm{H}(21)$ & 120 \\
\hline $\mathrm{C}(20)-\mathrm{C}(21)-\mathrm{H}(21)$ & 120 \\
\hline $\mathrm{C}(21)-\mathrm{C}(22)-\mathrm{N}(2)$ & 120 \\
\hline $\mathrm{C}(21)-\mathrm{C}(22)-\mathrm{H}(22)$ & 120 \\
\hline $\mathrm{N}(2)-\mathrm{C}(22)-\mathrm{H}(22)$ & 120 \\
\hline $\mathrm{C}(22)-\mathrm{N}(2)-\mathrm{C}(18)$ & 120 \\
\hline C(22)-N(2)-Pt(1) & $121(2)$ \\
\hline $\mathrm{C}(18)-\mathrm{N}(2)-\mathrm{Pt}(1)$ & $116(2)$ \\
\hline $\mathrm{C}(117)-\mathrm{C}(23)-\mathrm{H}(23 \mathrm{~A})$ & 110.6 \\
\hline $\mathrm{C}(117)-\mathrm{C}(23)-\mathrm{H}(23 \mathrm{~B})$ & 111.9 \\
\hline $\mathrm{H}(23 \mathrm{~A})-\mathrm{C}(23)-\mathrm{H}(23 \mathrm{~B})$ & 107.8 \\
\hline C(117)-C(23)-H(23C) & 111.2 \\
\hline $\mathrm{H}(23 \mathrm{~A})-\mathrm{C}(23)-\mathrm{H}(23 \mathrm{C})$ & 107.6 \\
\hline $\mathrm{H}(23 \mathrm{~B})-\mathrm{C}(23)-\mathrm{H}(23 \mathrm{C})$ & 107.5 \\
\hline
\end{tabular}




\begin{tabular}{|c|c|}
\hline $\mathrm{C}(25)-\mathrm{C}(24)-\mathrm{P}(3)$ & $118(4)$ \\
\hline $\mathrm{C}(25)-\mathrm{C}(24)-\mathrm{H}(24 \mathrm{~A})$ & 107.8 \\
\hline $\mathrm{P}(3)-\mathrm{C}(24)-\mathrm{H}(24 \mathrm{~A})$ & 107.8 \\
\hline $\mathrm{C}(25)-\mathrm{C}(24)-\mathrm{H}(24 \mathrm{~B})$ & 107.6 \\
\hline $\mathrm{P}(3)-\mathrm{C}(24)-\mathrm{H}(24 \mathrm{~B})$ & 107.7 \\
\hline $\mathrm{H}(24 \mathrm{~A})-\mathrm{C}(24)-\mathrm{H}(24 \mathrm{~B})$ & 107.1 \\
\hline $\mathrm{C}(24)-\mathrm{C}(25)-\mathrm{H}(25 \mathrm{~A})$ & 109.6 \\
\hline $\mathrm{C}(24)-\mathrm{C}(25)-\mathrm{H}(25 \mathrm{~B})$ & 109.4 \\
\hline $\mathrm{H}(25 \mathrm{~A})-\mathrm{C}(25)-\mathrm{H}(25 \mathrm{~B})$ & 109.5 \\
\hline C(24)-C(25)-H(25C) & 109.4 \\
\hline $\mathrm{H}(25 \mathrm{~A})-\mathrm{C}(25)-\mathrm{H}(25 \mathrm{C})$ & 109.5 \\
\hline $\mathrm{H}(25 \mathrm{~B})-\mathrm{C}(25)-\mathrm{H}(25 \mathrm{C})$ & 109.5 \\
\hline $\mathrm{C}(27)-\mathrm{C}(26)-\mathrm{P}(3)$ & $104(4)$ \\
\hline $\mathrm{C}(27)-\mathrm{C}(26)-\mathrm{H}(26 \mathrm{~A})$ & 110.9 \\
\hline $\mathrm{P}(3)-\mathrm{C}(26)-\mathrm{H}(26 \mathrm{~A})$ & 111.1 \\
\hline $\mathrm{C}(27)-\mathrm{C}(26)-\mathrm{H}(26 \mathrm{~B})$ & 111 \\
\hline $\mathrm{P}(3)-\mathrm{C}(26)-\mathrm{H}(26 \mathrm{~B})$ & 111.1 \\
\hline $\mathrm{H}(26 \mathrm{~A})-\mathrm{C}(26)-\mathrm{H}(26 \mathrm{~B})$ & 109.1 \\
\hline $\mathrm{C}(26)-\mathrm{C}(27)-\mathrm{H}(27 \mathrm{~A})$ & 112.3 \\
\hline $\mathrm{C}(26)-\mathrm{C}(27)-\mathrm{H}(27 \mathrm{~B})$ & 112.7 \\
\hline $\mathrm{H}(27 \mathrm{~A})-\mathrm{C}(27)-\mathrm{H}(27 \mathrm{~B})$ & 106.4 \\
\hline C(26)-C(27)-H(27C) & 112.5 \\
\hline $\mathrm{H}(27 \mathrm{~A})-\mathrm{C}(27)-\mathrm{H}(27 \mathrm{C})$ & 106.3 \\
\hline $\mathrm{H}(27 \mathrm{~B})-\mathrm{C}(27)-\mathrm{H}(27 \mathrm{C})$ & 106.2 \\
\hline $\mathrm{C}(41)-\mathrm{C}(40)-\mathrm{P}(2)$ & $123(4)$ \\
\hline $\mathrm{C}(41)-\mathrm{C}(40)-\mathrm{H}(40 \mathrm{~A})$ & 106.4 \\
\hline $\mathrm{P}(2)-\mathrm{C}(40)-\mathrm{H}(40 \mathrm{~A})$ & 106.4 \\
\hline $\mathrm{C}(41)-\mathrm{C}(40)-\mathrm{H}(40 \mathrm{~B})$ & 106.6 \\
\hline $\mathrm{P}(2)-\mathrm{C}(40)-\mathrm{H}(40 \mathrm{~B})$ & 106.7 \\
\hline $\mathrm{H}(40 \mathrm{~A})-\mathrm{C}(40)-\mathrm{H}(40 \mathrm{~B})$ & 106.6 \\
\hline C(40)-C(41)-H(41A) & 110.6 \\
\hline C(40)-C(41)-H(41B) & 111.1 \\
\hline $\mathrm{H}(41 \mathrm{~A})-\mathrm{C}(41)-\mathrm{H}(41 \mathrm{~B})$ & 108 \\
\hline C(40)-C(41)-H(41C) & 110.7 \\
\hline $\mathrm{H}(41 \mathrm{~A})-\mathrm{C}(41)-\mathrm{H}(41 \mathrm{C})$ & 108.1 \\
\hline $\mathrm{H}(41 \mathrm{~B})-\mathrm{C}(41)-\mathrm{H}(41 \mathrm{C})$ & 108.1 \\
\hline C(110)-C(43)-P(1) & $109(4)$ \\
\hline $\mathrm{C}(110)-\mathrm{C}(43)-\mathrm{H}(43 \mathrm{~A})$ & 110 \\
\hline $\mathrm{P}(1)-\mathrm{C}(43)-\mathrm{H}(43 \mathrm{~A})$ & 109.9 \\
\hline $\mathrm{C}(110)-\mathrm{C}(43)-\mathrm{H}(43 \mathrm{~B})$ & 109.9 \\
\hline $\mathrm{P}(1)-\mathrm{C}(43)-\mathrm{H}(43 \mathrm{~B})$ & 109.7 \\
\hline $\mathrm{H}(43 \mathrm{~A})-\mathrm{C}(43)-\mathrm{H}(43 \mathrm{~B})$ & 108.3 \\
\hline $\mathrm{C}(73)-\mathrm{C}(45)-\mathrm{P}(1)$ & $102(5)$ \\
\hline
\end{tabular}




\begin{tabular}{|c|c|}
\hline $\mathrm{C}(73)-\mathrm{C}(45)-\mathrm{H}(45 \mathrm{~A})$ & 111.3 \\
\hline $\mathrm{P}(1)-\mathrm{C}(45)-\mathrm{H}(45 \mathrm{~A})$ & 111.4 \\
\hline $\mathrm{C}(73)-\mathrm{C}(45)-\mathrm{H}(45 \mathrm{~B})$ & 111.4 \\
\hline $\mathrm{P}(1)-\mathrm{C}(45)-\mathrm{H}(45 \mathrm{~B})$ & 111.4 \\
\hline $\mathrm{H}(45 \mathrm{~A})-\mathrm{C}(45)-\mathrm{H}(45 \mathrm{~B})$ & 109.3 \\
\hline $\mathrm{C}(115)-\mathrm{C}(46)-\mathrm{H}(46 \mathrm{~A})$ & 109.6 \\
\hline $\mathrm{C}(115)-\mathrm{C}(46)-\mathrm{H}(46 \mathrm{~B})$ & 109.9 \\
\hline $\mathrm{H}(46 \mathrm{~A})-\mathrm{C}(46)-\mathrm{H}(46 \mathrm{~B})$ & 109.1 \\
\hline $\mathrm{C}(115)-\mathrm{C}(46)-\mathrm{H}(46 \mathrm{C})$ & 110 \\
\hline $\mathrm{H}(46 \mathrm{~A})-\mathrm{C}(46)-\mathrm{H}(46 \mathrm{C})$ & 109.1 \\
\hline $\mathrm{H}(46 \mathrm{~B})-\mathrm{C}(46)-\mathrm{H}(46 \mathrm{C})$ & 109.1 \\
\hline $\mathrm{O}(51)-\mathrm{C}(50)-\mathrm{O}(65)$ & $123(3)$ \\
\hline $\mathrm{O}(51)-\mathrm{C}(50)-\mathrm{C}(59)$ & $116(3)$ \\
\hline $\mathrm{O}(65)-\mathrm{C}(50)-\mathrm{C}(59)$ & $120(4)$ \\
\hline $\mathrm{C}(57)-\mathrm{C}(56)-\mathrm{C}(61)$ & 120 \\
\hline$C(57)-C(56)-C(62)$ & $124(3)$ \\
\hline$C(61)-C(56)-C(62)$ & $116(3)$ \\
\hline$C(58)-C(57)-C(56)$ & 120 \\
\hline $\mathrm{C}(58)-\mathrm{C}(57)-\mathrm{H}(57)$ & 120 \\
\hline $\mathrm{C}(56)-\mathrm{C}(57)-\mathrm{H}(57)$ & 120 \\
\hline $\mathrm{C}(59)-\mathrm{C}(58)-\mathrm{C}(57)$ & 120 \\
\hline $\mathrm{C}(59)-\mathrm{C}(58)-\mathrm{H}(58)$ & 120 \\
\hline $\mathrm{C}(57)-\mathrm{C}(58)-\mathrm{H}(58)$ & 120 \\
\hline$C(58)-C(59)-C(60)$ & 120 \\
\hline $\mathrm{C}(58)-\mathrm{C}(59)-\mathrm{C}(50)$ & $114(3)$ \\
\hline$C(60)-C(59)-C(50)$ & $126(3)$ \\
\hline$C(61)-C(60)-C(59)$ & 120 \\
\hline $\mathrm{C}(61)-\mathrm{C}(60)-\mathrm{H}(60)$ & 120 \\
\hline $\mathrm{C}(59)-\mathrm{C}(60)-\mathrm{H}(60)$ & 120 \\
\hline$C(60)-C(61)-C(56)$ & 120 \\
\hline $\mathrm{C}(60)-\mathrm{C}(61)-\mathrm{H}(61)$ & 120 \\
\hline $\mathrm{C}(56)-\mathrm{C}(61)-\mathrm{H}(61)$ & 120 \\
\hline $\mathrm{O}(1)-\mathrm{C}(62)-\mathrm{O}(63)$ & $125(4)$ \\
\hline $\mathrm{O}(1)-\mathrm{C}(62)-\mathrm{C}(56)$ & $115(4)$ \\
\hline $\mathrm{O}(63)-\mathrm{C}(62)-\mathrm{C}(56)$ & $118(4)$ \\
\hline $\mathrm{C}(68)-\mathrm{C}(67)-\mathrm{N}(1)$ & 120 \\
\hline $\mathrm{C}(68)-\mathrm{C}(67)-\mathrm{H}(67)$ & 120 \\
\hline N(1)-C(67)-H(67) & 120 \\
\hline$C(67)-C(68)-C(69)$ & 120 \\
\hline $\mathrm{C}(67)-\mathrm{C}(68)-\mathrm{H}(68)$ & 120 \\
\hline $\mathrm{C}(69)-\mathrm{C}(68)-\mathrm{H}(68)$ & 120 \\
\hline$C(70)-C(69)-C(68)$ & 120 \\
\hline $\mathrm{C}(70)-\mathrm{C}(69)-\mathrm{C}(11) \# 1$ & $120(2)$ \\
\hline
\end{tabular}




\begin{tabular}{|c|c|}
\hline $\mathrm{C}(68)-\mathrm{C}(69)-\mathrm{C}(11) \# 1$ & $119(2)$ \\
\hline$C(69)-C(70)-C(71)$ & 120 \\
\hline $\mathrm{C}(69)-\mathrm{C}(70)-\mathrm{H}(70)$ & 120 \\
\hline $\mathrm{C}(71)-\mathrm{C}(70)-\mathrm{H}(70)$ & 120 \\
\hline $\mathrm{N}(1)-\mathrm{C}(71)-\mathrm{C}(70)$ & 120 \\
\hline $\mathrm{N}(1)-\mathrm{C}(71)-\mathrm{H}(71)$ & 120 \\
\hline $\mathrm{C}(70)-\mathrm{C}(71)-\mathrm{H}(71)$ & 120 \\
\hline $\mathrm{C}(71)-\mathrm{N}(1)-\mathrm{C}(67)$ & 120 \\
\hline $\mathrm{C}(71)-\mathrm{N}(1)-\mathrm{Pt}(2)$ & $116.1(13)$ \\
\hline $\mathrm{C}(67)-\mathrm{N}(1)-\operatorname{Pt}(2)$ & $121.7(14)$ \\
\hline $\mathrm{C}(120)-\mathrm{C}(74)-\mathrm{P}(4)$ & $110(3)$ \\
\hline $\mathrm{C}(120)-\mathrm{C}(74)-\mathrm{H}(74 \mathrm{~A})$ & 108.8 \\
\hline $\mathrm{P}(4)-\mathrm{C}(74)-\mathrm{H}(74 \mathrm{~A})$ & 109.7 \\
\hline $\mathrm{C}(120)-\mathrm{C}(74)-\mathrm{H}(74 \mathrm{~B})$ & 110.4 \\
\hline $\mathrm{P}(4)-\mathrm{C}(74)-\mathrm{H}(74 \mathrm{~B})$ & 109.9 \\
\hline $\mathrm{H}(74 \mathrm{~A})-\mathrm{C}(74)-\mathrm{H}(74 \mathrm{~B})$ & 108.3 \\
\hline $\mathrm{C}(45)-\mathrm{C}(73)-\mathrm{H}(73 \mathrm{~A})$ & 120 \\
\hline C(45)-C(73)-H(73B) & 120 \\
\hline $\mathrm{H}(73 \mathrm{~A})-\mathrm{C}(73)-\mathrm{H}(73 \mathrm{~B})$ & 120 \\
\hline $\mathrm{C}(109)-\mathrm{C}(108)-\mathrm{H}(10 \mathrm{~A})$ & 110.7 \\
\hline $\mathrm{C}(109)-\mathrm{C}(108)-\mathrm{H}(10 \mathrm{~B})$ & 110 \\
\hline $\mathrm{H}(10 \mathrm{~A})-\mathrm{C}(108)-\mathrm{H}(10 \mathrm{~B})$ & 108.8 \\
\hline C(109)-C(108)-H(10C) & 109.8 \\
\hline $\mathrm{H}(10 \mathrm{~A})-\mathrm{C}(108)-\mathrm{H}(10 \mathrm{C})$ & 108.7 \\
\hline $\mathrm{H}(10 \mathrm{~B})-\mathrm{C}(108)-\mathrm{H}(10 \mathrm{C})$ & 108.8 \\
\hline C(108)-C(109)-P(3) & $135(4)$ \\
\hline $\mathrm{C}(108)-\mathrm{C}(109)-\mathrm{H}(10 \mathrm{D})$ & 103.6 \\
\hline $\mathrm{P}(3)-\mathrm{C}(109)-\mathrm{H}(10 \mathrm{D})$ & 103.4 \\
\hline C(108)-C(109)-H(10E) & 102.8 \\
\hline $\mathrm{P}(3)-\mathrm{C}(109)-\mathrm{H}(10 \mathrm{E})$ & 103.4 \\
\hline $\mathrm{H}(10 \mathrm{D})-\mathrm{C}(109)-\mathrm{H}(10 \mathrm{E})$ & 105.2 \\
\hline $\mathrm{C}(43)-\mathrm{C}(110)-\mathrm{H}(11 \mathrm{~A})$ & 112 \\
\hline $\mathrm{C}(43)-\mathrm{C}(110)-\mathrm{H}(11 \mathrm{~B})$ & 112.1 \\
\hline $\mathrm{H}(11 \mathrm{~A})-\mathrm{C}(110)-\mathrm{H}(11 \mathrm{~B})$ & 106.7 \\
\hline $\mathrm{C}(43)-\mathrm{C}(110)-\mathrm{H}(11 \mathrm{C})$ & 112.3 \\
\hline $\mathrm{H}(11 \mathrm{~A})-\mathrm{C}(110)-\mathrm{H}(11 \mathrm{C})$ & 106.8 \\
\hline $\mathrm{H}(11 \mathrm{~B})-\mathrm{C}(110)-\mathrm{H}(11 \mathrm{C})$ & 106.7 \\
\hline $\mathrm{C}(113)-\mathrm{C}(112)-\mathrm{P}(1)$ & $103(4)$ \\
\hline $\mathrm{C}(113)-\mathrm{C}(112)-\mathrm{H}(11 \mathrm{D})$ & 111.3 \\
\hline $\mathrm{P}(1)-\mathrm{C}(112)-\mathrm{H}(11 \mathrm{D})$ & 111.2 \\
\hline $\mathrm{C}(113)-\mathrm{C}(112)-\mathrm{H}(11 \mathrm{E})$ & 110.8 \\
\hline $\mathrm{P}(1)-\mathrm{C}(112)-\mathrm{H}(11 \mathrm{E})$ & 111.1 \\
\hline $\mathrm{H}(11 \mathrm{D})-\mathrm{C}(112)-\mathrm{H}(11 \mathrm{E})$ & 109.1 \\
\hline
\end{tabular}




\begin{tabular}{|c|c|}
\hline $\mathrm{C}(112)-\mathrm{C}(113)-\mathrm{H}(11 \mathrm{~F})$ & 115.1 \\
\hline $\mathrm{C}(112)-\mathrm{C}(113)-\mathrm{H}(11 \mathrm{G})$ & 114.8 \\
\hline $\mathrm{H}(11 \mathrm{~F})-\mathrm{C}(113)-\mathrm{H}(11 \mathrm{G})$ & 103.7 \\
\hline $\mathrm{C}(112)-\mathrm{C}(113)-\mathrm{H}(11 \mathrm{H})$ & 114.4 \\
\hline $\mathrm{H}(11 \mathrm{~F})-\mathrm{C}(113)-\mathrm{H}(11 \mathrm{H})$ & 103.6 \\
\hline $\mathrm{H}(11 \mathrm{G})-\mathrm{C}(113)-\mathrm{H}(11 \mathrm{H})$ & 103.8 \\
\hline C(116)-C(114)-P(2) & $104(2)$ \\
\hline C(116)-C(114)-H(11I) & 111 \\
\hline $\mathrm{P}(2)-\mathrm{C}(114)-\mathrm{H}(11 \mathrm{I})$ & 110.9 \\
\hline $\mathrm{C}(116)-\mathrm{C}(114)-\mathrm{H}(11 \mathrm{~J})$ & 110.9 \\
\hline $\mathrm{P}(2)-\mathrm{C}(114)-\mathrm{H}(11 \mathrm{~J})$ & 110.8 \\
\hline $\mathrm{H}(11 \mathrm{I})-\mathrm{C}(114)-\mathrm{H}(11 \mathrm{~J})$ & 108.9 \\
\hline $\mathrm{C}(46)-\mathrm{C}(115)-\mathrm{P}(2)$ & $105(4)$ \\
\hline $\mathrm{C}(46)-\mathrm{C}(115)-\mathrm{H}(11 \mathrm{~K})$ & 110.7 \\
\hline $\mathrm{P}(2)-\mathrm{C}(115)-\mathrm{H}(11 \mathrm{~K})$ & 110.7 \\
\hline $\mathrm{C}(46)-\mathrm{C}(115)-\mathrm{H}(11 \mathrm{~L})$ & 111 \\
\hline $\mathrm{P}(2)-\mathrm{C}(115)-\mathrm{H}(11 \mathrm{~L})$ & 111 \\
\hline $\mathrm{H}(11 \mathrm{~K})-\mathrm{C}(115)-\mathrm{H}(11 \mathrm{~L})$ & 108.9 \\
\hline $\mathrm{C}(114)-\mathrm{C}(116)-\mathrm{H}(11 \mathrm{M})$ & 110.6 \\
\hline$C(114)-C(116)-H(11 N)$ & 110.5 \\
\hline $\mathrm{H}(11 \mathrm{M})-\mathrm{C}(116)-\mathrm{H}(11 \mathrm{~N})$ & 108.5 \\
\hline C(114)-C(116)-H(11O) & 110.3 \\
\hline $\mathrm{H}(11 \mathrm{M})-\mathrm{C}(116)-\mathrm{H}(11 \mathrm{O})$ & 108.4 \\
\hline $\mathrm{H}(11 \mathrm{~N})-\mathrm{C}(116)-\mathrm{H}(11 \mathrm{O})$ & 108.5 \\
\hline $\mathrm{C}(23)-\mathrm{C}(117)-\mathrm{P}(4)$ & $92(6)$ \\
\hline $\mathrm{C}(23)-\mathrm{C}(117)-\mathrm{H}(11 \mathrm{P})$ & 113.4 \\
\hline $\mathrm{P}(4)-\mathrm{C}(117)-\mathrm{H}(11 \mathrm{P})$ & 113.3 \\
\hline $\mathrm{C}(23)-\mathrm{C}(117)-\mathrm{H}(11 \mathrm{Q})$ & 112.9 \\
\hline P(4)-C(117)-H(11Q) & 113.3 \\
\hline H(11P)-C(117)-H(11Q) & 110.7 \\
\hline C(119)-C(118)-P(4) & $112(3)$ \\
\hline C(119)-C(118)-H(11R) & 109 \\
\hline $\mathrm{P}(4)-\mathrm{C}(118)-\mathrm{H}(11 \mathrm{R})$ & 109.1 \\
\hline C(119)-C(118)-H(11S) & 109.4 \\
\hline P(4)-C(118)-H(11S) & 109.2 \\
\hline $\mathrm{H}(11 \mathrm{R})-\mathrm{C}(118)-\mathrm{H}(11 \mathrm{~S})$ & 107.9 \\
\hline $\mathrm{C}(118)-\mathrm{C}(119)-\mathrm{H}(11 \mathrm{~T})$ & 110.7 \\
\hline C(118)-C(119)-H(11U) & 111.1 \\
\hline H(11T)-C(119)-H(11U) & 107.9 \\
\hline $\mathrm{C}(118)-\mathrm{C}(119)-\mathrm{H}(11 \mathrm{~V})$ & 110.9 \\
\hline $\mathrm{H}(11 \mathrm{~T})-\mathrm{C}(119)-\mathrm{H}(11 \mathrm{~V})$ & 108 \\
\hline $\mathrm{H}(11 \mathrm{U})-\mathrm{C}(119)-\mathrm{H}(11 \mathrm{~V})$ & 108 \\
\hline C(74)-C(120)-H(12A) & 122.2 \\
\hline
\end{tabular}




$\begin{array}{lr}\text { C(74)-C(120)-H(12B) } & 123.9 \\ \text { H(12A)-C(120)-H(12B) } & 92.5 \\ \text { C(74)-C(120)-H(12C) } & 124.8 \\ \text { H(12A)-C(120)-H(12C) } & 92.8 \\ \text { H(12B)-C(120)-H(12C) } & 91.5 \\ \text { O(3S)-S(1S)-O(2S) } & 116(3) \\ \text { O(3S)-S(1S)-O(1S) } & 114(3) \\ \text { O(2S)-S(1S)-O(1S) } & 113(3) \\ \text { O(3S)-S(1S)-C(1S) } & 107(3) \\ \text { O(2S)-S(1S)-C(1S) } & 102(3) \\ \text { O(1S)-S(1S)-C(1S) } & 103(3) \\ \text { F(1S)-C(1S)-F(2S) } & 104(4) \\ \text { F(1S)-C(1S)-F(3S) } & 103(4) \\ \text { F(2S)-C(1S)-F(3S) } & 102(4) \\ \text { F(1S)-C(1S)-S(1S) } & 117(3) \\ \text { F(2S)-C(1S)-S(1S) } & 115(3) \\ \text { F(3S)-C(1S)-S(1S) } & 115(3) \\ \text { O(4S)-S(2S)-O(5S) } & 115(3) \\ \text { O(4S)-S(2S)-O(6S) } & 113(3) \\ \text { O(5S)-S(2S)-O(6S) } & 113(3) \\ \text { O(4S)-S(2S)-C(2S) } & 107(3) \\ \text { O(5S)-S(2S)-C(2S) } & 105(3) \\ \text { O(6S)-S(2S)-C(2S) } & 103(3) \\ \text { F(4S)-C(2S)-F(6S) } & 101(4) \\ \text { F(4S)-C(2S)-F(5S) } & 101(4) \\ \text { F(6S)-C(2S)-F(5S) } & 100(4) \\ \text { F(4S)-C(2S)-S(2S) } & 118(3) \\ \text { F(6S)-C(2S)-S(2S) } & 118(3) \\ \text { F(5S)-C(2S)-S(2S) } & 116(3) \\ & \\ & \end{array}$

Symmetry transformations used to generate equivalent atoms:

$\# 1-x+1 / 2,-y+1 / 2,-z+1 / 2$

\section{References}

S1. Muratsugu, S.; Yamaguchi, A.; Yokota, G. I.; Maeno, T.; Tada, M. Tuning the Structure and Catalytic Activity of $\mathrm{Ru}$ Nanoparticle Catalysts by Single 3d Transition-Metal Atoms in Ru12Metalloporphyrin Precursors. Chem. Commun. 2018, 54, 4842-4845.

S2. Stang, P. J.; Cao, D. H.; Saito, S.; Arif, A. M. Self-Assembly of Cationic, Tetranuclear, Pt (II) and $\mathrm{Pd}$ (II) Macrocyclic Squares. X-Ray Crystal Structure of $\left[\mathrm{Pt}^{2+}(\mathrm{Dppp})\left(4,4^{\prime}-\mathrm{Bipyridyl}\right)_{2} \bullet \mathrm{OSO}_{2} \mathrm{CF}_{3}\right]_{4} . J$. Am. Chem. Soc. 1995, 117, 6273-6283. 